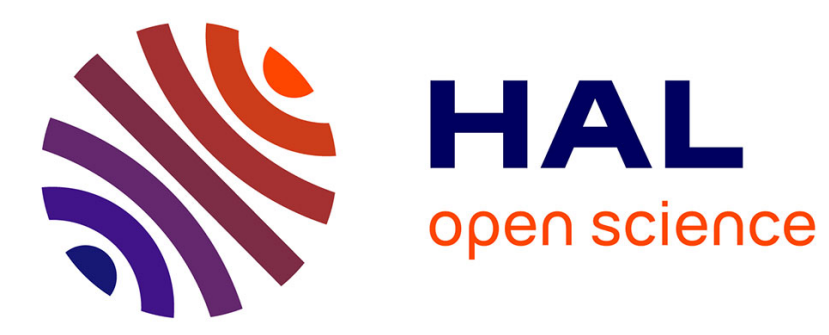

\title{
Tackling the Challenges of Enzymatic (Bio)Fuel Cells
}

Xinxin Xiao, Hong-Qi Xia, Ranran Wu, Lu Bai, Lu Yan, Edmond Magner, Serge Cosnier, Elisabeth Lojou, Zhiguang Zhu, Aihua Liu

\section{To cite this version:}

Xinxin Xiao, Hong-Qi Xia, Ranran Wu, Lu Bai, Lu Yan, et al.. Tackling the Challenges of Enzymatic (Bio)Fuel Cells. Chemical Reviews, 2019, 10.1021/acs.chemrev.9b00115 . hal-02167914

\section{HAL Id: hal-02167914 https://hal-amu.archives-ouvertes.fr/hal-02167914}

Submitted on 28 Jun 2019

HAL is a multi-disciplinary open access archive for the deposit and dissemination of scientific research documents, whether they are published or not. The documents may come from teaching and research institutions in France or abroad, or from public or private research centers.
L'archive ouverte pluridisciplinaire HAL, est destinée au dépôt et à la diffusion de documents scientifiques de niveau recherche, publiés ou non, émanant des établissements d'enseignement et de recherche français ou étrangers, des laboratoires publics ou privés. 
Tackling the challenges of enzymatic (bio)fuel cells

Xinxin Xiao ${ }^{\dagger, l, \perp}$, Hong-qi Xia ${ }^{\dagger, \perp, \Sigma}$, Ranran $\mathrm{Wu}^{\ddagger, \perp}$, Lu Bai ${ }^{\dagger}$, Lu Yan ${ }^{\dagger}$, Edmond Magner, Serge



${ }^{\dagger}$ Institute for Biosensing, and College of Life Sciences, Qingdao University, 308 Ningxia

Road, Qingdao 266071, China

Tianjin Institute of Industrial Biotechnology, Chinese Academy of Sciences, 32 West 7th

Road, Tianjin Airport Economic Area, Tianjin 300308, China

${ }^{\zeta}$ College of Chemistry \& Chemical Engineering, Qingdao University, 308 Ningxia Road,

Qingdao 266071, China

${ }^{\gamma}$ School of Pharmacy, Medical College, Qingdao University, Qingdao, 266021, China

${ }^{\S}$ Aix Marseille Univ, CNRS, BIP, Bioénergétique et Ingénierie des Protéines UMR7281,

Institut de Microbiologie de la Méditerranée, IMM, FR 3479, 31, chemin Joseph Aiguier

13402 Marseille Cedex 20, France

'Department of Chemical Sciences, School of Natural Sciences and Bernal Institute,

University of Limerick, Limerick V94 T9PX, Ireland

${ }^{\Delta}$ Université Grenoble-Alpes, DCM UMR 5250, F-38000 Grenoble, France

${ }^{\eta}$ Département de Chimie Moléculaire, UMR CNRS, DCM UMR 5250, F-38000 Grenoble,

France

${ }^{\perp}$ These authors contributed equally to this work.

*To whom correspondence should be addressed: E-mails: liuah@qdu.edu.cn (A.L.);

zhu_zg@tib.cas.cn (Z.Z.); lojou@imm.cnrs.fr (E.L.)

${ }^{\Sigma}$ Present address: School of Biomedical Engineering, Sun Yat-sen University, Guangzhou 510006, China 


\section{ABSTRACT}

The ever-increasing demands for clean and sustainable energy sources combined with rapid advances in bio-integrated portable or implantable electronic devices have stimulated intensive research activities in enzymatic (bio)fuel cells (EFCs). The use of renewable biocatalysts, the utilization of abundant green, safe, and high energy density fuels, together with the capability of working at modest and biocompatible conditions, make EFCs promising as next generation alternative power sources. However, the main challenges (low energy density, relatively low power density, poor operational stability and limited voltage output) hinder future applications of EFCs. This review aims at exploring the underlying mechanism of EFCs and providing possible practical strategies, methodologies and insights to tackle of these issues. Firstly, this review summarizes approaches in achieving high energy densities in EFCs, particularly, employing enzyme cascades for the deep/complete oxidation of fuels. Secondly, strategies for increasing power densities in EFCs, including increasing enzyme activities, facilitating electron transfers, employing nanomaterials, and designing more efficient enzyme-electrode interfaces, are described. The potential of EFCs/(super)capacitor combination is discussed. Thirdly, the review evaluates a range of strategies for improving the stability of EFCs, including the use of different enzyme immobilization approaches, tuning enzyme properties, designing protective matrixes, and using microbial surface displaying enzymes. Fourthly, approaches for the improvement of the cell voltage of EFCs are highlighted. Finally, future developments and a prospective on EFCs are envisioned. 


\section{CONTENTS}







1.1 Enzymatic (bio)fuel cells (EFCs), general considerations ................................. 5

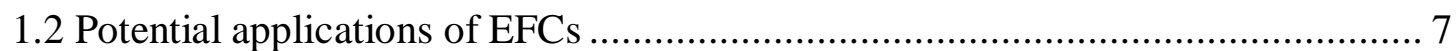

1.3 Identification of main challenges in EFCs .................................................. 9

2. Strategies for achieving high energy density in EFCs ...................................... 24

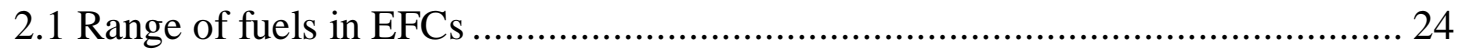

2.2 Enzyme cascades for the deep/complete oxidation of fuels ............................. 26

3. Strategies for increasing power density in EFCs ............................................ 31

3.1 Evaluation of different power output results................................................. 31



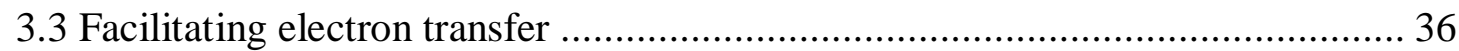



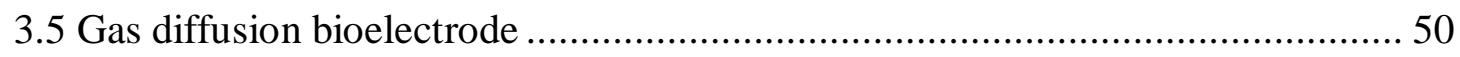

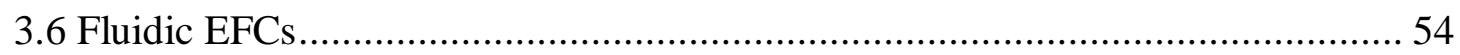

3.7 Combined EFCs/(super)capacitor devices ................................................... 56

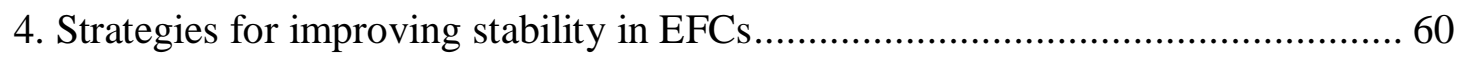









4.3 Microbial surface displayed enzymes as biocatalysts to enhance EFCs' stability 70

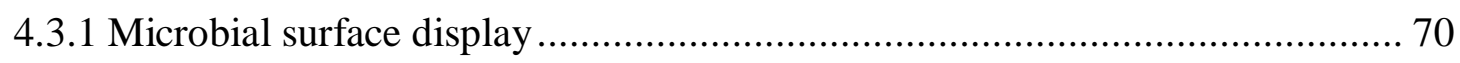

4.3.2. Efficient EFCs based on microbial surface displayed enzyme as biocatalysts . 71

4.4 Strategies for enzyme protection against $\mathrm{O}_{2}$ and reactive oxygen species (ROS) 74



5. Approaches for the improvement of EFCs' cell voltage .................................... 81

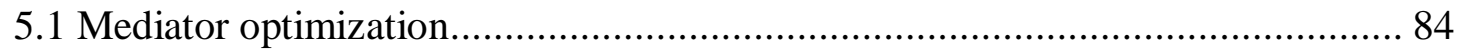



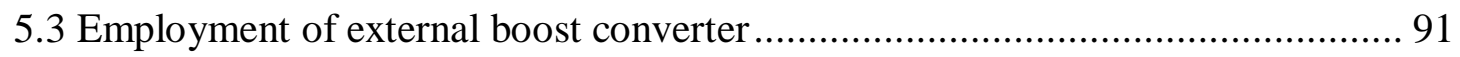

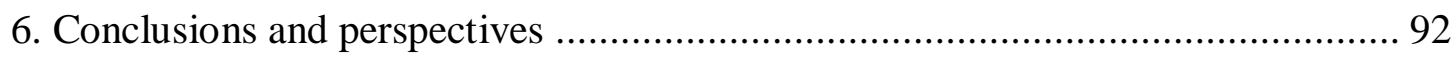


Acknowledgements .



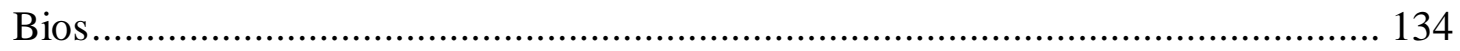




\section{Introduction}

\subsection{Enzymatic (bio)fuel cells (EFCs), general considerations}

The uneven geographical distribution of fuels associated with the increasingly serious effects of environmental pollution provides the driving force for the pursuit of green and sustainable energy sources. To this end, fuel cells are considered environmentally friendly electrochemical devices to directly convert chemical energy into electrical energy without intermediate steps. ${ }^{1}$ In general, conventional fuel cells use noble metals (e.g. platinum, ruthenium, palladium, etc.) and/or their alloys as catalysts for the oxidation of pure fuels (e.g. hydrogen, methanol) at the anode and the reduction of the oxidant (e.g. oxygen) at the cathode, which work in optimized basic and/or acid electrolytes, resulting in a very high efficiency. However, noble metals are costly and, more importantly, are non-renewable resources only available in few countries in the world. The use of electrolytes at extremes of $\mathrm{pH}$, accompanying with the requirement for expensive membranes to separate reactions into individual compartments, poses additional challenges.

In addition to the need for clean and renewable energy, recent rapid advances in bio-integrated implantable or portable electronic devices underline the urgent need to develop technologies that can harvest energy from biological sources. $^{2}$ A range of potential applications in microelectronic, biomedical, and sensor devices have inspired research in energy conversion systems utilizing sources such as body heat, muscle stretching, blood flow, walking or running, etc. ${ }^{3}$ However, low levels of biocompatibility and durability pose potential health and safety concerns, raising significant challenges in the successful development of such devices.

EFCs are a subclass of fuel cells employing redox enzymes as catalysts ${ }^{4-9}$. The concept of an EFC was first described by Yahiro and co-workers in $1964 .^{10}$ Depending on emerging possible applications, EFCs have been designed in various configurations that may be quite 
different from the traditional fuel cell stacks. However, they all retain the same key components. Similar to other fuel cells, EFCs consist of a two-electrode cell separated by a proton conducting medium, which can also be an electrolyte (Figure 1): using appropriate redox enzymes, fuels are oxidized at the bioanode, electrons flow through the external electric circuit to the biocathode, where the oxidants, usually oxygen ${ }^{11}$ or peroxides ${ }^{12}$, are reduced to water. Using bioelectrocatalysts, EFCs have several advantages. Firstly, the catalyst is renewable. Redox enzymes can be extracted from a wide range of living organisms in a renewable manner. Secondly, fuels can be diverse. In principle, sugars ${ }^{13}$, alcohols ${ }^{14}$, organic $\operatorname{acids}^{15}$, hydrogen ${ }^{16}$, and mixtures of these materials that can be digested by living organisms, can be used as fuels for EFCs. Thirdly, the operational conditions are very mild and safe. The properties of enzymatic reactions enable EFCs to operate at physiological $\mathrm{pH}$, room temperature and ambient pressure, although the recent use of stable extremophilic enzymes offers the possibility to work at temperatures of up to $85{ }^{\circ} \mathrm{C}$ or at a $\mathrm{pH}$ value as low as $2 .{ }^{17,18}$ In addition, redox enzymes provide exceptional specificity towards their natural substrates, thus allowing the assembly of the bioanode and biocathode in a single membrane-less cell and the miniaturization of EFCs. ${ }^{19}$ Another consequence of high enzyme specificity is that EFCs can use fuels without the need for intensive purification steps. Finally, EFCs can be considered as disposable systems as the components can potentially be biologically degraded. These properties demonstrate the potential use of EFCs in next-generation green power source for a range of applications.



Figure 1. Schematic drawing of a typical EFC consisting of a bioanode and a biocathode. 
Bioelectrocatalysis, in which the electrons involved in an enzymatic reaction are collected at an electrode surface, is a key element of EFCs. Due to the size and structure of the enzymes, electron transfer (ET) within the enzyme and between the enzyme and the electrode is specific. In general, the electron transfer mechanisms between enzymes and electrodes are classified into two types: mediated electron transfer (MET) and direct electron transfer (DET) $)^{8,20,21}$. In a MET-type system, extrinsic redox-active compounds such as ferrocene ${ }^{22}$, methyl viologen, ABTS are used as redox mediators to shuttle electrons between the enzyme cofactor (for example, glucose oxidase (GOx) uses flavin adenine dinucleotide (FAD) as the cofactor) and the electrode ${ }^{23}$. In this case, the redox enzyme catalyzes the oxidation or reduction of the mediator as a co-substrate. The reverse transformation (regeneration) of the mediator occurs on the electrode surface. The use of small, low molecular weight electron mediators that require low overpotentials can be beneficial as they can enable rapid rates of electron transfer between an enzyme and an electrode with low power losses. However, the cost, stability, selectivity and ability to exchange electron in the immobilized state of such mediators must also be considered. In contrast, in a DET-type system, fast electron transfer to or from a solid electrode occurs through an intrinsic electron relay system in the protein ${ }^{20}$ (e.g. iron-sulfur clusters $^{24,25}$, heme groups ${ }^{26,27}$ or copper sites ${ }^{28,29}$ ).

\subsection{Potential applications of EFCs}

The early development of EFCs focussed on obtaining electrical energy mainly through the oxidation of glucose or other organic fuels in living organisms, in order to drive implantable electronic devices. The power output of an average human body is approximately $100 \mathrm{~W}$, and the constant presence and availability of the fuel from the body provides sufficient support for running $\mathrm{EFCs}^{30}$. The oxygen or other oxidant supply for the biocathode in such EFCs is important as EFCs are implanted in a relatively closed system. Since the first total surgical implantation of a EFC in a rat in $2010,{ }^{31}$ several recent studies on implantable EFCs have been reported. In particular, biocathodes modified by chitosan implanted in a rat can exhibit a stability of up to 167 days ${ }^{32}$. Cyborg lobsters with partially implanted EFCs were able to 
power an electrical watch and a pacemaker ${ }^{33}$, while a fully implanted EFC in rats can power a light-emitting diode (LED), or a digital thermometer ${ }^{34}$. Mountable EFCs using trehalose in insect hemolymph were also successfully demonstrated ${ }^{35}$. However, special attention should be focused on solving sterilization and biocompatibility issues, as well as their poor operational stabilities, before such implantable or mountable EFCs can become practical, especially when used in human patients ${ }^{36-40}$.

During the past decades, significant improvements in the power output and stability of EFCs have been achieved, paving the way for the use of EFCs to power portable electronic devices such as music players, cellphones, sensors, and even laptops. Many studies have demonstrated the use of EFCs as power sources for LEDs or for digital clocks ${ }^{41-43}$. In 2007, Sony demonstrated that a music player could be powered by a stack of EFCs. Later, they demonstrated the operation of a toy car using a glucose-fueled EFC. However, there are considerable challenges to the development of these devices as cost effective power sources that can be manufactured on a large scale ${ }^{44}$.

Another potential application of EFCs is emerging with the rapid development of wearable electronic devices that are shaping our life in healthcare, communication, entertainment, etc. Such wearable electronic devices can potentially be powered by EFCs operating using external fuels, or directly from fuel in the body ${ }^{45,46}$. In contrast to implantable EFCs, non-invasive EFCs have attracted considerable interest ${ }^{47}$. Several wearable EFCs have been constructed using lactate in sweat or in tears as the fuel and generate reasonable power outputs $^{48,49}$. Such wearable EFCs need to be flexible in order to withstand frequently bending or folding. A number of layer-by-layer or printed EFCs have been developed that exhibit good flexibility, high performances, and a potential of low fabrication $\operatorname{costs}^{50,51}$. In the following sub-section, the main challenges of these EFCs will be discussed and the particular limitations of each application will be described. 

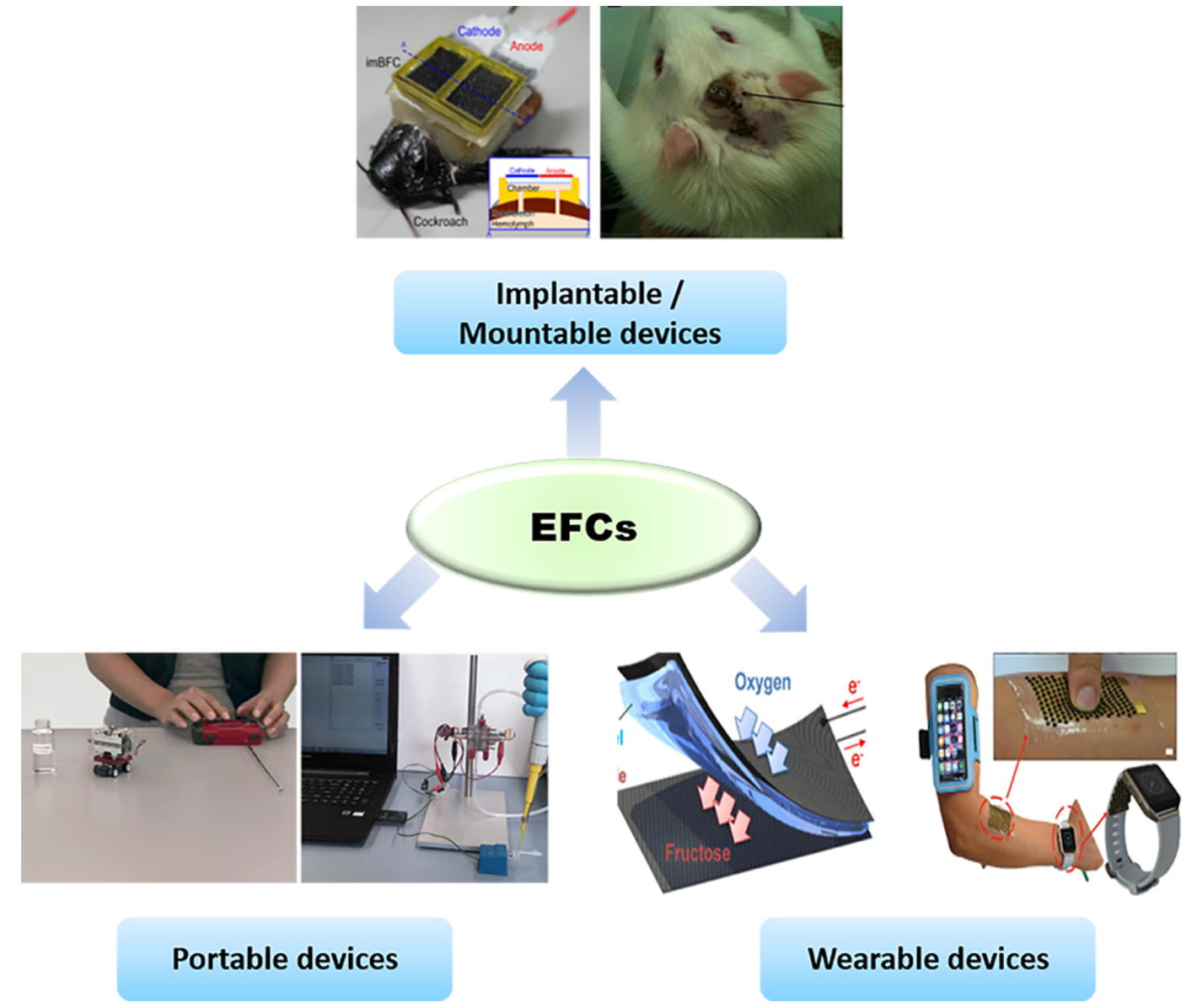

Figure 2. Possible applications of EFCs activating implantable, portable and wearable devices. Reprinted with permission ${ }^{34,35,50,52-54}$ Copyright 2013, 2016, 2018 Elsevier; Copyright 2009, 2017 Royal Society of Chemistry; Copyright 2013 Nature Publishing Group.

\subsection{Identification of main challenges in EFCs}

The development of EFCs faces four significant challenges: inability to completely oxidize fuels, low power density, poor operational stability, and limited voltage output. Although each of these challenges can be addressed from multiple aspects, some potential solutions are too complex to implement and may also be detrimental factors in terms of other aspects of cell performance. Due to the complexity of these challenges, a systematic analysis should be made to identify the key reasons behind each challenge and to then carefully evaluate multiple possible solutions ${ }^{55}$. 
Firstly, most EFCs employ one or two oxidoreductases. Depending on the fuel, complete oxidation cannot be achived, leading to low efficiency and energy density, critical parameters for all power sources. As one of the principle advantages of EFCs mentioned above, sugars or alcohols can store much higher energy per weight or volume than most secondary batteries ${ }^{56}$. However, exploitation of such energy storage potential requires a series of cascade reactions that oxidize the fuel in a step by step manner. The use of one or two enzymes makes it impossible to implement the complete oxidation of a fuel. As a consequence, the cost of fuels, inhibition (by products or intermediates), the difficulties in reusing cells, and decreased power output pose significant obstacles to the successful development of EFCs.

The issue of low power output of EFCs has been a major problem that constrains potential use to applications ${ }^{57}$. Compared with metal-catalyzed fuel cells or lithium-ion batteries, the power output produced from EFCs is significantly lower. The power densities of the majority of EFCs lie in the range of $1-1000 \mu \mathrm{W} \mathrm{cm}$, with few surpassing $1 \mathrm{~mW} \mathrm{~cm}^{-2}$ (Table 1). A principal reason for this is that the active site of enzyme is buried inside a large insulating protein moiety. Typically, a $0.5 \mathrm{mg} \mathrm{cm}^{-2} \mathrm{Pt}$ loading on the electrode of a metal-catalyzed fuel cell represents $2.5 \mu \mathrm{mol}$ of catalyst $\mathrm{cm}^{-2}$, while the catalyst loading for a GOx or laccase (Lac)-immobilized electrode is only at the level of $10^{-6}$ to $10^{-1} \mu \mathrm{mol} \mathrm{cm}{ }^{-2} 58,59$ in an EFC depending on the electrode of choice. The overall reaction rate per volume or area, in terms of power density, for enzyme biocatalysts is decreased by orders of magnitude. In addition, the availability of the fuel may become a limiting factor in power generation, especially for implantable EFCs, which may have limited oxidant supply ${ }^{60}$. Tackling this issue requires a combinatorial strategy of engineering electrode materials, enzymes, and their interfaces as well as smart configuration design ${ }^{4}$. 
Table 1. Full EFCs with a maximum power density $\left(\mathrm{P}_{\max }\right)$ greater than $1 \mathrm{~mW} \mathrm{~cm}$

\begin{tabular}{|c|c|c|c|c|c|c|c|}
\hline \multicolumn{8}{|c|}{ Glucose/ $\mathrm{O}_{2}$ EFCs } \\
\hline No. & Bioanode & Biocathode & Note & $\begin{array}{l}\mathrm{P}_{\max } \\
(\mathrm{mW} \\
\left.\mathrm{cm}^{-2}\right)\end{array}$ & $\begin{array}{l}\text { OCV } \\
(\mathrm{V})\end{array}$ & Stability & Ref. \\
\hline 1 & $\begin{array}{l}\mathrm{CF} / \mathrm{GDH} / \mathrm{DI} / \mathrm{VK}_{3} / \mathrm{NADH}(1.5 \mathrm{~mm} \\
\text { thick); } \\
\text { MET; } \\
400 \mathrm{mM} \text { glucose }\end{array}$ & $\begin{array}{l}\mathrm{CF} / \mathrm{K}_{3}\left[\mathrm{Fe}(\mathrm{CN})_{6}\right] / \mathrm{BOD} ; \\
\text { MET; } \\
\text { Air-breathing }\end{array}$ & $\begin{array}{l}\text { Two-compartm } \\
\text { ent; limited by } \\
\text { anode }\end{array}$ & 1.45 & 0.8 & $\begin{array}{l}\text { Continuous } \\
\text { operation over } 2 \\
\text { h }\end{array}$ & 52 \\
\hline 2 & $\begin{array}{l}\mathrm{CF} / \mathrm{GDH} / \mathrm{DI} / \mathrm{ANQ} / \mathrm{NADH} ; \\
\mathrm{MET} ; \\
400 \mathrm{mM} \text { glucose }\end{array}$ & $\begin{array}{l}\mathrm{CF} / \mathrm{K}_{3}\left[\mathrm{Fe}(\mathrm{CN})_{6}\right] / \mathrm{BOD} ; \\
\mathrm{MET} \\
\text { Air-breathing }\end{array}$ & $\begin{array}{l}\text { Two-compartm } \\
\text { ent; limited by } \\
\text { cathode }\end{array}$ & 3 & 0.8 & $\mathrm{n} / \mathrm{a}$ & 61 \\
\hline 3 & $\begin{array}{l}\text { MWCNTs-PEDOT yarn/Os-complex } \\
\text { modified polymer(I)/GOx; } \\
\text { MET; } \\
60 \mathrm{mM} \text { glucose }\end{array}$ & $\begin{array}{l}\text { MWCNTs-PEDOT } \\
\text { yarn/Os-complex modified } \\
\text { polymer(II)/BOD; } \\
\text { MET; } \\
\mathrm{O}_{2} \text {-saturated }\end{array}$ & $\begin{array}{l}\text { One-compartm } \\
\text { ent; limited by } \\
\text { cathode }\end{array}$ & 2.18 & 0.7 & $\begin{array}{l}83 \% \text { remaining } \\
\text { after } 24 \mathrm{~h}\end{array}$ & 62 \\
\hline 4 & $\begin{array}{l}\mathrm{HPC} / \mathrm{AQ} 2 \mathrm{~S} / \mathrm{DI} / \mathrm{NAD}^{+} / \mathrm{GDH} ; \\
\mathrm{MET} ; \\
800 \mathrm{mM} \text { glucose }\end{array}$ & $\begin{array}{l}\mathrm{CF} / \mathrm{K}_{3}\left[\mathrm{Fe}(\mathrm{CN})_{6}\right] / \mathrm{BOD} ; \\
\mathrm{MET} \\
\text { Air-breathing }\end{array}$ & $\begin{array}{l}\text { Two-compartm } \\
\text { ent; limited by } \\
\text { anode }\end{array}$ & 1 & 0.8 & $\begin{array}{l}\text { Can be used } \\
\text { for }>10 \text { cycles }\end{array}$ & 63 \\
\hline 5 & $\begin{array}{l}\text { GCE/MWCNTs/NQ-4-LPEI/GDH; } \\
\text { MET; }\end{array}$ & $\begin{array}{l}\text { CP/anthracene-MWCNTs/ } \\
\text { BOD; }\end{array}$ & $\begin{array}{l}\text { One-compartm } \\
\text { ent; limited by }\end{array}$ & 2.3 & 0.86 & $\begin{array}{l}\text { Potential } \\
\text { decreased from }\end{array}$ & 64 \\
\hline
\end{tabular}


Stirred $100 \mathrm{mM}$ glucose

6

MWCNTs/NQ/GOx/catalase;

MET;

$50 \mathrm{mM}$ glucose

7 MWCNTs/GOx/catalase(3 mm

thick);

MET;

$50 \mathrm{mM}$ glucose

\begin{tabular}{|c|c|c|c|c|c|c|c|}
\hline & \multicolumn{7}{|c|}{$\mathrm{H}_{2} / \mathrm{O}_{2} \mathrm{EFCs}$} \\
\hline & Bioanode & Bio-/cathode & Note & $\begin{array}{l}\mathrm{P}_{\max } \\
(\mathrm{mW} \\
\left.\mathrm{cm}^{-2}\right)\end{array}$ & $\begin{array}{l}\text { OCV } \\
(\mathrm{V})\end{array}$ & Stability & Ref. \\
\hline 8 & $\begin{array}{l}\mathrm{CNF} / \text { Aquifex aeolicus }(\mathrm{Aa})[\mathrm{NiFe}] \\
\text { hydrogenase; } \\
\text { DET; } \\
\mathrm{H}_{2} \text {-saturated }\end{array}$ & $\begin{array}{l}\text { CNF/Bacillus pumilus }(B p) \\
\text { BOD; } \\
\text { DET; } \\
\mathrm{O}_{2} \text {-saturated }\end{array}$ & $\begin{array}{l}\text { Two-compartm } \\
\text { ent; limited by } \\
\text { cathode }\end{array}$ & $\begin{array}{l}1.5 \text { at } \\
60^{\circ} \mathrm{C}\end{array}$ & 1.06 & $\begin{array}{l}\text { Decreased by } \\
60 \% \text { at } 0.5 \mathrm{~V} \\
\text { after } 24 \mathrm{~h}\end{array}$ & 65 \\
\hline 9 & $\begin{array}{l}\text { WPCC/KB/ Desulfovibrio } \\
\text { vulgaris }(D v)[\mathrm{NiFe}] \text { hydrogenase; } \\
\text { DET; } \\
\mathrm{H}_{2} \text { diffusion electrode }\end{array}$ & $\begin{array}{l}\text { WPCC/KB/Myrothecium } \\
\text { verrucaria }(M v) \mathrm{BOD} ; \\
\text { DET; } \\
\text { Air-breathing }\end{array}$ & $\begin{array}{l}\text { Dual } \\
\text { gas-diffusion } \\
\text { type; } \\
\text { One-compartm } \\
\text { ent; limited by }\end{array}$ & 6.1 & 1.12 & $\mathrm{n} / \mathrm{a}$ & 16 \\
\hline
\end{tabular}

0.86 to $0.71 \mathrm{~V}$

after $24 \mathrm{~h}$

operation

$\mathrm{O}_{2}$-saturated anode
MWCNTs/Lac;

DET
$60 \%$ decrease

over 7 days'

storage

One-compartm

1.3

0.95

Stable for 1

month ent

$\begin{array}{llll}\begin{array}{l}\text { One-compartm } \\ \text { ent; limited by } \\ \text { anode }\end{array} & 1.54 & 0.76 & \begin{array}{l}60 \% \text { decrease } \\ \text { over 7 days' } \\ \text { storage }\end{array} \\ \text { One-compartm } & 1.3 & 0.95 & \begin{array}{l}\text { Stable for 1 } \\ \text { month }\end{array}\end{array}$


cathode

10

CMC/E. coli hydrogenase-1;

DET

$11 \mathrm{CF} / \mathrm{CNTs} / \mathrm{A} a[\mathrm{NiFe}]$ hydrogenase;

DET;

$\mathrm{H}_{2}$-saturated

12

$\mathrm{WPCC} / \mathrm{KB} / D v[\mathrm{NiFe}]$ hydrogenase;

DET;

$100 \% \mathrm{H}_{2}$

13 Carbon cloth $/ D v[\mathrm{NiFe}]$

hydrogenase/

$\mathrm{P}\left(\mathrm{N}_{3}\right.$ MA-BA-GMA)-vio/

P(GMA-BA-PEGMA)-vio;

MET;

$100 \% \mathrm{H}_{2}$
$\mathrm{CMC} / M v \mathrm{BOD}$;

DET

CF/CNTs/BpBOD;

DET;

$\mathrm{O}_{2}$-saturated

$\mathrm{WPCC} / \mathrm{KB} / \mathrm{M} v \mathrm{BOD}$;

DET;

$100 \% \mathrm{O}_{2}$

Carbon cloth $/ M v \mathrm{BOD}$;

DET;

$100 \% \mathrm{O}_{2}$

In a $78 \%$
$\mathrm{H}_{2}-22 \%$ air
mixture;
One-compartm
ent; limited by
cathode

1.67 (per

anode

area)

1.068

Retained 90\%

output after

continuously

working for $24 \mathrm{~h}$

Two-compartm

ent; limited by

anode

Dual

gas-diffusion;

not a real EFC

assembly

Dual

gas-diffusion;

One-compartm

ent; limited by

anode
1.7 at

$50{ }^{\circ} \mathrm{C}$

8.4

$1.025 \%$ loss after 17

h operation

$1.14 \mathrm{n} / \mathrm{a}$

3.6

1.13 Retained 46\%

69

output after $24 \mathrm{~h}$

continuous

operation 


\begin{tabular}{|c|c|c|c|c|c|c|c|}
\hline & Bioanode & Bio-/cathode & Note & $\begin{array}{l}\mathrm{P}_{\max } \\
(\mathrm{mW} \\
\left.\mathrm{cm}^{-2}\right)\end{array}$ & $\begin{array}{l}\text { OCV } \\
(\mathrm{V})\end{array}$ & Stability & Ref. \\
\hline 14 & $\begin{array}{l}\mathrm{CP} / \mathrm{CCG} / \mathrm{FDH} ; \\
\mathrm{DET} ; \\
500 \mathrm{mM} \text { fructose }\end{array}$ & $\begin{array}{l}\mathrm{CP} / \mathrm{KB} / M v \mathrm{BOD} \\
\mathrm{DET} \\
\text { Air-breathing }\end{array}$ & $\begin{array}{l}\text { DET-type EFC; } \\
\text { One-compartm } \\
\text { ent; limited by } \\
\text { anode }\end{array}$ & 2.6 & 0.79 & $\mathrm{n} / \mathrm{a}$ & 70 \\
\hline 15 & $\begin{array}{l}\text { CNTs/FDH; } \\
\text { DET; } \\
200 \mathrm{mM} \text { fructose }\end{array}$ & $\begin{array}{l}\mathrm{CNTs} / \mathrm{Lac} \\
\mathrm{DET} \\
\mathrm{O}_{2} \text {-saturated }\end{array}$ & $\begin{array}{l}\text { One-compartm } \\
\text { ent; limited by } \\
\text { cathode }\end{array}$ & 1.8 & 0.77 & $\begin{array}{l}\text { Retained } 84 \% \\
\text { output after } 24 \mathrm{~h} \\
\text { continuous } \\
\text { operation }\end{array}$ & 71 \\
\hline 16 & $\begin{array}{l}\text { GCE/MWCNTs/CPPy/FDH; } \\
\text { DET; } \\
100 \mathrm{mM} \text { fructose }\end{array}$ & $\begin{array}{l}\text { GCE/MWCNTs/CPPy/AB } \\
\text { TS/Lac; } \\
\text { MET; } \\
\mathrm{O}_{2} \text {-saturated }\end{array}$ & $\begin{array}{l}\text { One-compartm } \\
\text { ent; limited by } \\
\text { cathode }\end{array}$ & 2.1 & 0.59 & $\begin{array}{l}60 \% \text { loss after } 1 \\
\text { week operation }\end{array}$ & 72 \\
\hline \multicolumn{8}{|c|}{ Formate/ $\mathrm{O}_{2} \mathrm{EFCs}$} \\
\hline & Bioanode & Bio-/cathode & Note & $\begin{array}{l}\mathrm{P}_{\max } \\
(\mathrm{mW} \\
\left.\mathrm{cm}^{-2}\right)\end{array}$ & $\begin{array}{l}\text { OCV } \\
(\mathrm{V})\end{array}$ & Stability & Ref. \\
\hline 17 & $\begin{array}{l}\text { NG/AuNPs/FoDH; } \\
5 \mathrm{mM} \mathrm{NAD}^{+} \text {and } 50 \mathrm{mM} \text { formic acid }\end{array}$ & $\begin{array}{l}\text { NG/AuNPs/Lac; } \\
\text { MET; } \\
0.5 \text { mM ABTS }\end{array}$ & $\begin{array}{l}\text { One-compartm } \\
\text { ent; limited by } \\
\text { cathode }\end{array}$ & 1.96 & 0.95 & $\begin{array}{l}\text { Not directly } \\
\text { measured }\end{array}$ & 73 \\
\hline 18 & WPCC/KB/viologen-functionalized & $\mathrm{WPCC} / \mathrm{KB} / M v$ & One-compartm & 12 & 0.78 & $\mathrm{n} / \mathrm{a}$ & 15 \\
\hline
\end{tabular}


polymer/FoDH;

MET;

$300 \mathrm{mM}$ formate
BOD/ABTS;

MET;

$\mathrm{O}_{2}$ diffusion electrode ent;

Thick

electrode;

limited by

anode

\begin{tabular}{|c|c|c|c|c|c|c|c|}
\hline & \multicolumn{7}{|c|}{ Sucrose/ $\mathrm{O}_{2} \mathrm{EFCs}$} \\
\hline & Bioanode & Bio-/cathode & Note & $\begin{array}{l}\mathrm{P}_{\max } \\
(\mathrm{mW} \\
\left.\mathrm{cm}^{-2}\right)\end{array}$ & $\begin{array}{l}\text { OCV } \\
(\mathrm{V})\end{array}$ & Stability & Ref. \\
\hline 19 & $\begin{array}{l}\text { Carbon } \\
\text { felt/CNTs/TTF/GOx/FDH/MUT/INV } \\
; \\
\text { MET; } \\
50 \mathrm{mM} \text { sucrose }\end{array}$ & $\begin{array}{l}\text { Carbon } \\
\text { felt/CNTs/ABTS/BOD; } \\
\text { MET; } \\
\mathrm{O}_{2} \text {-saturated }\end{array}$ & $\begin{array}{l}\text { Deep oxidation } \\
\text { of sucrose }\end{array}$ & 2.9 & 0.69 & $\begin{array}{l}\text { Bioanode } \\
\text { displayed good } \\
\text { stability for } 0.5 \\
\text { h }\end{array}$ & 74 \\
\hline & \multicolumn{7}{|c|}{ Ethanol/ $/ \mathrm{O}_{2} \mathrm{EFCs}$} \\
\hline & Bioanode & Bio-/cathode & Note & $\begin{array}{l}\mathrm{P}_{\max } \\
(\mathrm{mW} \\
\left.\mathrm{cm}^{-2}\right)\end{array}$ & $\begin{array}{l}\text { OCV } \\
(\mathrm{V})\end{array}$ & Stability & Ref. \\
\hline 20 & $\begin{array}{l}\mathrm{MDB} / \mathrm{AuNPs} / \mathrm{gel} / \mathrm{ADH} ; \\
\mathrm{MET} ; \\
1 \mathrm{mM} \mathrm{NAD}^{+} \text {and } 1 \mathrm{mM} \text { ethanol }\end{array}$ & $\begin{array}{l}\text { AuNPs/gel/Lac; } \\
\text { DET; } \\
\text { Air-equilibrated }\end{array}$ & $\begin{array}{l}\text { One-compartm } \\
\text { ent }\end{array}$ & 1.56 & 0.86 & $\begin{array}{l}80 \% \text { loss after } \\
36 \text { days }\end{array}$ & 75 \\
\hline 21 & $\begin{array}{l}\mathrm{PAN} \\
\text { nanofiber/Au/Super-P/ADH/NAD } / \mathrm{D} \\
\mathrm{I} / / \mathrm{VK}_{3} ;\end{array}$ & $\begin{array}{l}\text { PAN } \\
\text { nanofiber/Au/Super-P/Lac/ } \\
\text { ABTS; }\end{array}$ & $\begin{array}{l}\text { Two-compartm } \\
\text { ent; limited by } \\
\text { cathode }\end{array}$ & 1.6 & 0.99 & $\begin{array}{l}\text { Pronounced loss } \\
\text { of } \mathrm{NAD}^{+}\end{array}$ & 76 \\
\hline
\end{tabular}



MET;
MET;
$\sim 69 \mathrm{mM}$ ethanol
$\mathrm{O}_{2}$-saturated

Abbreviations: CF: Carbon fiber; GDH: glucose dehydrogenase; DI: diaphorase; $\mathrm{VK}_{3}$ : vitamin $\mathrm{K}_{3}$; NADH: $\beta$-Nicotinamide adenine dinucleotide disodium salt (reduced form); BOD: bilirubin oxidase; ANQ: 2-amino- 1,4-naphthoquinone; Lac: laccase; MET: mediated electron transfer; MWCNTs: multi-walled carbon nanotubes; PEDOT: poly(3,4-ethylenedioxythiophene); Os-complex modified polymer(I):

poly $\left.\left.(N \text {-vinylimidazole)-[Os(4,4'-dimethoxy-2,2'-bipyridine })_{2} \mathrm{Cl}\right]\right)^{+/ 2+} ;$ Os-complex modified polymer(II): poly(acryl

5 amide)-poly( $N$-vinylimidazole)-[Os(4,4'-dichloro-2,2'-bipyridine $\left.\left.)_{2}\right]\right)^{+/ 2+}$; HPC: hierarchical porous carbon; AQ2S: anthraquinone-2-sulfonate; NQ-4-LPEI: naphthoquinone(NQ)-modified linear polyethyleneimine; CP: carbon paper; CNF: carbon nanofibers; WPCC: water proof carbon paper; KB: Ketjen black; CMC: compacted mesoporous carbon; P(N 3 MA-BA-GMA)-vio: poly(3-azido-propyl methacrylate-co-butyl acrylate-co-glycidyl methacrylate)-viologen; P(GMA-BA-PEGMA)-vio: poly(glycidyl methacrylate-co-butyl acrylate-co-poly(ethylene glycol) methacrylate)-viologen; CCG: carbon cryogel; CPPy: cellulose/polypyrrole composite; NG: nitrogen-doped graphene; FoDH: formate dehydogenase; ABTS: 2,2'-azinobis(3-ethylbenzothiazoline-6-sulfonate);

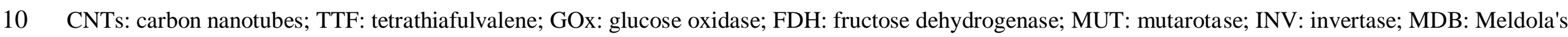
blue; AuNPs: gold nanoparticles; PAN: polyacrylonitrile. 
Moreover, as a typical enzyme-catalyzed system, EFCs suffer from poor operational stability, resulting in short lifetimes and higher costs. ${ }^{77,78}$ Instability arises not just with the enzyme, but also arises from the use of cofactors such as nicotinamide adenine dinucleotide $\left(\mathrm{NAD}^{+}\right)$, adenosine triphosphate (ATP), and coenzyme A (CoA), which are necessary for many redox enzymes, and of other components that include mediators. The complexity of biological systems can pose additional detrimental effects on the stability of EFCs, such as biofouling of the electrode in implantable EFCs, or enzyme inhibition by $\mathrm{O}_{2}$ for $\mathrm{H}_{2} / \mathrm{O}_{2}$ EFCs. In contrast to relatively stable proton exchange membrane fuel cells and metal-based batteries that can last for years, or microbial fuel cells (MFCs) utilising self-reproducing microorganisms that can be reused for months, the majority of EFCs can operate only for hours or days ${ }^{79-81}$.

For almost all reported EFCs, the voltage at which usable power can be extracted is below the minimal requirement to power commercially available electronic devices. This drawback is inevitable as, from a thermodynamic point of view, the maximum redox potential gap between two electrodes in most biological fuel cells (e.g. $\sim 1.18 \mathrm{~V}$ for glucose/ $\mathrm{O}_{2} \mathrm{EFCs}$ with two-electron oxidation of glucose) is much less than that of lithium-based batteries (e.g. 4.2 V) ${ }^{36}$. In many cases, the involvement of electron mediators leads to additional decreases in the voltage output of EFCs. In addition, the actual voltage output of EFCs is decreased by factors such as ohmic and concentration losses. Such losses can also depress the current output, and further reduce the overall power output.

Significant developments in EFCs have occurred since the first report in 1964. A number of reviews on EFCs have recently been published ${ }^{3,7,39,79,82-88}$, the majority of which have focused on either the electrode materials ${ }^{40,87-95}$, enzyme immobilization ${ }^{40,90,96-98}$, bioelectrocatalysis $^{8,11,99-101}$ or their applications ${ }^{39,40,45,47,86,88,102}$. Table 2 summarizes a list of the reviews reported since 2015 on bioelectrodes and EFCs. This review identifies the main scientific challenges hindering the development of EFCs, low energy and power densities, 
poor operational stability as well as limited voltage output, and summarizes the corresponding approaches to solve them.

Table 2. Reviews relevant to bioelectrodes and EFCs since 2015

\begin{tabular}{|c|c|c|}
\hline Topic & Title & Year and ref. \\
\hline \multirow{3}{*}{\multicolumn{3}{|c|}{ Wired Enzymes in Mesoporous Materials: A 2015}} \\
\hline & & \\
\hline & & \\
\hline & Graphene Based Enzymatic Bioelectrodes and & $2015^{93}$ \\
\hline & Biofuel Cells & \\
\hline & 3D Graphene Biocatalysts for Development of & $2015^{104}$ \\
\hline & Enzymatic Biofuel Cells: A Short Review & \\
\hline & Tailoring Biointerfaces for Electrocatalysis & $2016^{105}$ \\
\hline & Magneto-Switchable Electrodes and Electrochemical & $2016^{106}$ \\
\hline & Systems & \\
\hline & Application of Carbon Fibers to Flexible Enzyme & $2016^{81}$ \\
\hline & Electrodes & \\
\hline & Paper Electrodes for Bioelectrochemistry: & $2016^{107}$ \\
\hline & Biosensors and Biofuel Cells & \\
\hline & An Overview of Dealloyed Nanoporous Gold in & $2016^{108}$ \\
\hline & Bioelectrochemistry & \\
\hline & Enzymatic Biofuel Cells on Porous Nanostructures & $2016^{92}$ \\
\hline & Conformational Changes of Enzymes and Aptamers & $2016^{109}$ \\
\hline & Immobilized on Electrodes & \\
\hline & Progress on Implantable Biofuel Cell: Nano-Carbon & $2016^{40}$ \\
\hline & Functionalization for Enzyme Immobilization & \\
\hline & Enhancement & \\
\hline & Enzymatic Reactions in Confined Environments & $2016^{110}$ \\
\hline
\end{tabular}


Biomimetic and Bioinspired Approaches for Wiring $2016^{78}$

Enzymes to Electrode Interfaces

Nanostructured Material-Based Biofuel Cells: 2017

Recent Advances and Future Prospects

Nanostructured Inorganic Materials at Work in 2017

Electrochemical Sensing and Biofuel Cells

Carbon Felt Based-Electrodes for Energy and $2017^{111}$

Environmental Applications: A Review

Advanced Materials for Printed Wearable $2017^{112}$

Electrochemical Devices: A Review

Recent Advance in Fabricating Monolithic 3D $2017^{95}$

Porous Graphene and Their Applications in

Biosensing and Biofuel Cells

Enzyme Immobilization on Nanoporous Gold: A $2017^{113}$

Review

Recent Developments in High Surface Area $2017^{114}$

Bioelectrodes for Enzymatic Fuel Cells

Graphene and Graphene Oxide: Functionalization $2018^{115}$

and Nano-Bio-Catalytic System for Enzyme

Immobilization and Biotechnological Perspective

Molecular Engineering of the Bio/Nano-Interface for $2018^{116}$

Enzymatic Electrocatalysis in Fuel Cells

Buckypaper Bioelectrodes: Emerging Materials for $2018^{117}$

Implantable and Wearable Biofuel Cells

Recent Applications of Bacteriophage-Based 2019

Electrodes: A Mini-Review

Redox polymers

Current Trends in Redox Polymers for Energy and $2016^{119}$ 
Medicine

Redox Polymers in Bioelectrochemistry: Common 2017

Playgrounds and Novel Concepts

Gas diffusion electrodes

Application of Gas Diffusion Electrodes in $2016^{121}$

Bioelectrochemical Syntheses and Energy

Conversion

Gas Diffusion Bioelectrodes

$2017^{102}$

Enzyme engineering

The Use of Engineered Protein Materials in $2016^{122}$

Electrochemical Devices

Enzyme cascades

Oxidative Bioelectrocatalysis: From Natural $2016^{123}$

Metabolic Pathways to Synthetic Metabolons and

Minimal Enzyme Cascades

Enzyme Cascades in Biofuel Cells

$2017^{124}$

Electron transfer

processes

Direct Enzymatic Bioelectrocatalysis: Differentiating $2017^{125}$

Between Myth and Reality

Mathematical Modeling of Nonlinear $2017^{126}$

Reaction-Diffusion Processes in Enzymatic Biofuel

Cells

Protein Bioelectronics: A Review of What We Do $2018^{127}$

and Do Not Know

Controlling Redox Enzyme Orientation at Planar 2018

Electrodes

Electrochemistry of Surface-Confined Enzymes: 2018 ${ }^{129}$ 
Inspiration, Insight and Opportunity for Sustainable

Biotechnology

Direct Electron Transfer of Enzymes Facilitated by $2019^{130}$

Cytochromes

Sugar oxidation

Direct Electron Transfer (DET) Mechanism of FAD $2018^{131}$

Dependent Dehydrogenase Complexes $\sim$ From the

Elucidation of Intra- and Inter-Molecular Electron

Transfer Pathway to the Construction of Engineered

DET Enzyme Complexes

Direct Electron Transfer of Dehydrogenases for $2018^{132}$

Development of 3rd Generation Biosensors and

Enzymatic Fuel Cells

Enzymatic oxidation of

$\mathrm{H}_{2}$

Guiding Principles of Hydrogenase Catalysis 2016 ${ }^{133}$

Instigated and Clarified by Protein Film

Electrochemistry

New Perspectives in Hydrogenase Direct $2017^{134}$

Electrochemistry

Enzymatic reduction of

$\mathrm{O}_{2}$

Recent Progress in Oxygen-Reducing Laccase $2015^{99}$

Biocathodes for Enzymatic Biofuel Cells

Oxygen $\quad$ Electroreduction $\quad$ Versus $2016^{135}$

Bioelectroreduction: Direct Electron Transfer

Approach

Laccase: A Multi-Purpose Biocatalyst at the $2017^{136}$ 
Forefront of Biotechnology

$\mathrm{O}_{2}$ Reduction in Enzymatic Biofuel Cells

Application of Eukaryotic and Prokaryotic Laccases $2018^{138}$

in Biosensor and Biofuel Cells: Recent Advances

and Electrochemical Aspects

Biocapacitor

Biocapacitor: A Novel Principle for Biosensors

$2016^{139}$

Biosupercapacitors

$2017^{140}$

Microfluidic biofuel cells

Generating Electricity on Chips: Microfluidic $2018^{141}$

Biofuel Cells in Perspective

Implantable enzymatic

fuel cells

Tear Based Bioelectronics

$2016^{142}$

Quo Vadis, Implanted Fuel Cell?

$2017^{60}$

Challenges for Successful Implantation of Biofuel $2018^{30}$

Cells

Implantable Energy-Harvesting Devices

$2018^{143}$

Wearable enzymatic fuel

cells

Wearable Biofuel Cells: A Review

$2016^{45}$

Review-Wearable Biofuel Cells: Past, Present and 2017

Future

Wearable Bioelectronics: Enzyme-Based 2018

Body-Worn Electronic Devices

Biofuel Cells - Activation of Micro- and $2018^{44}$

Macro-Electronic Devices

Wearable Biofuel Cells Based on the Classification $2019^{145}$ 
of Enzyme for High Power Outputs and Lifetimes

Self-powered system

Energy Harvesting from the Animal/Human Body $2017^{146}$

for Self-Powered Electronics

Recent Advances in the Construction of Biofuel $2018^{147}$

Cells Based Self-Powered Electrochemical

Biosensors: A Review

Energy-Autonomous Biosensing Platform Using 2018 ${ }^{148}$

Supply-Sensing CMOS Integrated Sensor and

Biofuel Cell for Next-Generation Healthcare Internet

of Things

Self-Powered Bioelectrochemical Devices $\quad 2018^{149}$

Enzymatic Fuel Cells: Towards Self-Powered $2018^{150}$

Implantable and Wearable Diagnostics

Self-Powered Biosensors $2018^{151}$

Enzymatic fuel cells

Enzymatic Biofuel Cells: 30 Years of Critical 2015

Advancements

Recent Advances on Enzymatic Glucose/Oxygen $2016^{8}$

and Hydrogen/Oxygen Biofuel Cells: Achievements

and Limitations

$\mathrm{H}_{2} / \mathrm{O}_{2}$ Enzymatic Fuel Cells: From Proof-of-Concept $2017^{88}$

to Powerful Devices

Beyond the Hype Surrounding Biofuel Cells: What's $2018^{9}$

the Future of Enzymatic Fuel Cells? 


\section{Strategies for achieving high energy density in EFCs}

Like other types of fuel cells, the available energy density of an EFC is dependent on the product of the chemical energy stored in the fuel and the faradaic efficiency. The faradaic efficiency is described by:

$$
\eta_{\mathrm{F}}=\int \mathrm{I} \times \mathrm{dt} /\left(\mathrm{c}_{\text {fuel }} \times \mathrm{V} \times \mathrm{n} \times \mathrm{F}\right)
$$

where $\eta_{\mathrm{F}}=$ faradaic efficiency, $\mathrm{I}=$ current, $\mathrm{t}=$ reaction time, $\mathrm{c}_{\text {fuel }}=$ concentration of fuel, $\mathrm{V}=$ reaction volume, $\mathrm{n}=$ number of electrons generated per fuel, and $\mathrm{F}=$ Faraday constant (96,485 C per mole). Clearly, it is desirable to combine high-energy-density fuels with high faradaic efficiencies to achieve high energy density EFCs.

\subsection{Range of fuels in EFCs}

EFCs harness power from living and renewable biological sources. Compared with traditional rare metal-catalyzed fuel cells that are predominantly powered by hydrogen or methanol, the fuel diversity of EFCs has been greatly broadened to many organic compounds which are common intermediates metabolized in living organisms or are the main components of biomass. Although a wide variety of fuels can be used for EFCs, their energy density, cost, availability, and toxicity all need to be considered.

Hydrogen has one of the highest energy density values per mass and has been widely used in traditional fuel cells. As a clean fuel that can be produced from biomass or water splitting, it can also be used in an EFC catalyzed by hydrogenases. ${ }^{16,88,152-154}$ Storage and distribution of $\mathrm{H}_{2}$ have been the subject of intensive research, enabling the use of $\mathrm{H}_{2}$ in a safe manner. Alternatively, formic acid is a stable hydrogen carrier and has been used to power some EFCs due to its advantages of high volumetric capacity $\left(53 \mathrm{~g} \mathrm{H}_{2} \mathrm{~L}^{-1}\right)$, low toxicity and flammability under ambient conditions. Methanol is another promising alternative to hydrogen as a fuel because it is accessible and easy to transport and store, although it is toxic for human beings if ingested. It has a nearly 3-fold higher volumetric energy density than that of formic acid. 
Furthermore, the theoretical maximum voltage for a methanol/oxygen fuel cell $(1.19 \mathrm{~V})$ is close to that for a $\mathrm{H}_{2} / \mathrm{O}_{2}$ fuel cell $(1.23 \mathrm{~V})^{155}$. Although ethanol is rarely considered as a fuel source in fuel cells, it has some advantages, such as low cost, non-toxicity and wide availability. In addition, ethanol is a renewable energy source that can be generated through fermentation of agricultural products. As another prospective fuel, glycerol has many desirable qualities and is abundant since it is a by-product of biodiesel production. Properties such as low toxicity, low flammability, extremely low vapor pressure and high energy density make glycerol very appealing as an energy source ${ }^{156}$. Pyruvate, a key intermediate from the glycolysis pathway, has also been used as a fuel in EFCs ${ }^{157}$. Finally, it is noteworthy that the most commonly used fuels are sugars as they are inexpensive, abundant, renewable, and safe to use. They can be derived from lignocellulosic biomass (ca. $1 \times 10^{11}$ tons/year globally), which can be locally grown and are more evenly distributed than fossil fuels. Among various sugars, glucose is the most widely used fuel in EFCs, and glucose-based EFCs are particularly suited for implantable applications due to its presence in blood at reasonable concentrations $(\mathrm{mM})$. Many other sugars including xylose, fructose, sucrose, and polysaccharides such as maltodextrin have also been used in $\mathrm{EFCs}^{56,158,159}$.

Full exploitation of the energy stored in a substrate can provide high energy densities, a key advantage of EFCs compared with commonly available batteries. Theoretically, glucose possesses an energy density of $4,125 \mathrm{Wh} \mathrm{L}^{-1}$ releasing 24 electrons per glucose molecule to produce carbon dioxide and water. Hence, the complete enzymatic oxidation of the glucose units of a $15 \%$ maltodextrin solution indicates that the energy-storage density of the EFC can be as high as $596 \mathrm{Ah} \mathrm{kg}^{-1}$, which is an order of magnitude higher than that of lithium-ion batteries and primary batteries ${ }^{42}$. Glycerol has an even higher energy density $\left(6,260 \mathrm{Wh} \mathrm{L}^{-1}\right)$ compared to glucose, or to ethanol $\left(5,442 \mathrm{Wh} \mathrm{L}^{-1}\right)$, methanol $\left(4,047 \mathrm{Wh} \mathrm{L}^{-1}\right)$, making it a very attractive fuel. Notably, pyruvate also has a high energy density $\left(4,594 \mathrm{Wh} \mathrm{L}^{-1}\right)$, and requires fewer enzymes than glucose for complete oxidation. 


\subsection{Enzyme cascades for the deep/complete oxidation of fuels}

When building an EFC, maximizing both energy density and power density is of crucial importance. The majority of EFCs utilizes a single enzyme to perform partial oxidation of a fuel (i.e. glucose, lactate, pyruvate or ethanol), but the complete oxidation of the majority of fuels requires several enzymes to use the energy available in the fuel ${ }^{82}$. As a relevant example, when the degree of catalytic oxidation as well as the maximum allowable fuel concentration are taken into account, the energy density of an ethanol fuel cell based on $20 \% \mathrm{v} / \mathrm{v}$ ethanol with incomplete oxidation to acetic acid decreases from 5,442 to $363 \mathrm{Wh} \mathrm{L}^{-1}{ }^{156}$. Therefore, one of the key issues in developing high-energy-density EFCs is the successful design of multi-enzyme systems that can completely oxidize the fuel in order to increase the overall energy efficiency.

Living cells are able to completely oxidize complex fuels into carbon dioxide and water through the tricarboxylic acid (TCA) cycle, a crucial metabolic pathway ${ }^{160}$. In the cycle, acetyl-CoA is oxidized to carbon dioxide and water, generating the reduced forms of nicotinamide-adenine dinucleotide $(\mathrm{NADH})$ and flavin adenine dinucleotide $\left(\mathrm{FADH}_{2}\right)$ and chemical energy in the form of ATP. Several fuels can be fed into the TCA cycle, and each requires different sets of enzyme cascades. One of these fuels is glucose, which can be oxidized through the glycolysis pathway to pyruvate, which is subsequently oxidized to acetyl-CoA by pyruvate dehydrogenase. Lactate can also enter into the TCA cycle after dehydrogenation by lactate dehydrogenase (LDH). Ethanol has also been used as a substrate by introducing alcohol dehydrogenase $(\mathrm{ADH})$, aldehyde dehydrogenase (AldDH), and S-acetyl CoA synthetase to oxidize ethanol into acetyl-CoA ${ }^{160}$. By mimicking the natural TCA pathway, several EFCs have been developed that can completely oxidize glucose, ethanol, pyruvate, and lactate. For instance, in an ethanol/ $\mathrm{O}_{2} \mathrm{EFC}$, dehydrogenases along with non-energy producing enzymes necessary for the cycle were immobilized in cascades onto a carbon electrode in a tetrabutylammonium bromide modified Nafion membrane, generating an 8.71-fold increase in power density compared to a single enzyme (ADH)-based ethanol/air 
EFC ${ }^{161}$. In another mitochondria-based fuel cell consisting of all the enzymes involved in the TCA cycle, pyruvate was converted to acetyl-CoA by a pyruvate dehydrogenase and further oxidized by the enzyme cascade. A 4.6-fold increase in power density was observed when using intact mitochondria as compared to that using an individual enzyme in the TCA cycle ${ }^{162}$. It should be noted however that the increased power densities obtained in these systems 161,162 are still significantly lower than the theoretically expected values.

In addition to mimic the natural pathways, in vitro synthetic pathways to completely oxidize fuels have been described. The first EFC based on enzyme cascades that can completely transform alcohols was demonstrated in $1998^{155}$, where three NAD-dependent dehydrogenases including $\mathrm{ADH}, \mathrm{AldDH}$ and formate dehydrogenase (FoDH) were employed to fully oxidize methanol to carbon dioxide and water (Figure 3). Six electrons per methanol molecule were collected at the bioanode when $\mathrm{NADH}$ was re-oxidized into $\mathrm{NAD}^{+}$with the assistance of redox mediators. However, this complete oxidation process relied on enzymes in solution rather than immobilized at the electrode surface. Later, Minteer et al. ${ }^{124}$ conducted a series of studies of enzyme immobilization based on this pathway, including encapsulation within hydrophobically modified Nafion ${ }^{163}$ and self-assembled enzymatic hydrogel ${ }^{164}$. An EFC based on the two-step oxidation of ethanol to acetate mediated by ADH and AldDH was described $^{161}$.



Figure 3. The oxidation of methanol to $\mathrm{CO}_{2}$ is catalyzed by NAD-dependent alcohol-(ADH), aldehyde-(AldDH), and formate-dehydrogenases (FoDH) (shown within the box). Regeneration of $\mathrm{NAD}^{+}$is accomplished electro-enzymatically with an enzyme coupled to the anode via a redox mediator. Reprinted with permission ${ }^{155}$ with modification. Copyright 1998 Elsevier. 
Presently, the growth of the biodiesel industry has greatly increased the production of glycerol $^{165}$. As already stated above, employing glycerol as a fuel for bioelectricity generation is a promising route. However, catalysts based on precious metals can only remove four electrons of a total of sixteen electrons available for the complete oxidation of glycerol ${ }^{156}$, leading to a low energy density. In contrast, EFCs have the ability to exploit the energy of glycerol by employing an enzyme cascade to oxidize glycerol in a stepwise pathway. It has been demonstrated that a three-enzyme cascade containing pyrroloquinoline quinone (PQQ)-dependent ADH, AldDHs and oxalate oxidase immobilized within a Nafion membrane can accomplish the complete oxidation of glycerol, with a fuel utilization efficiency up to $98.9 \%{ }^{156}$. More recently, a hybrid enzymatic and abiotic catalytic system that combined free oxalate oxidase and 4-amino-TEMPO was constructed to electrochemically oxidize glycerol at a carbon electrode, collecting as many as 16 electrons per molecule of glycerol ${ }^{166}$.

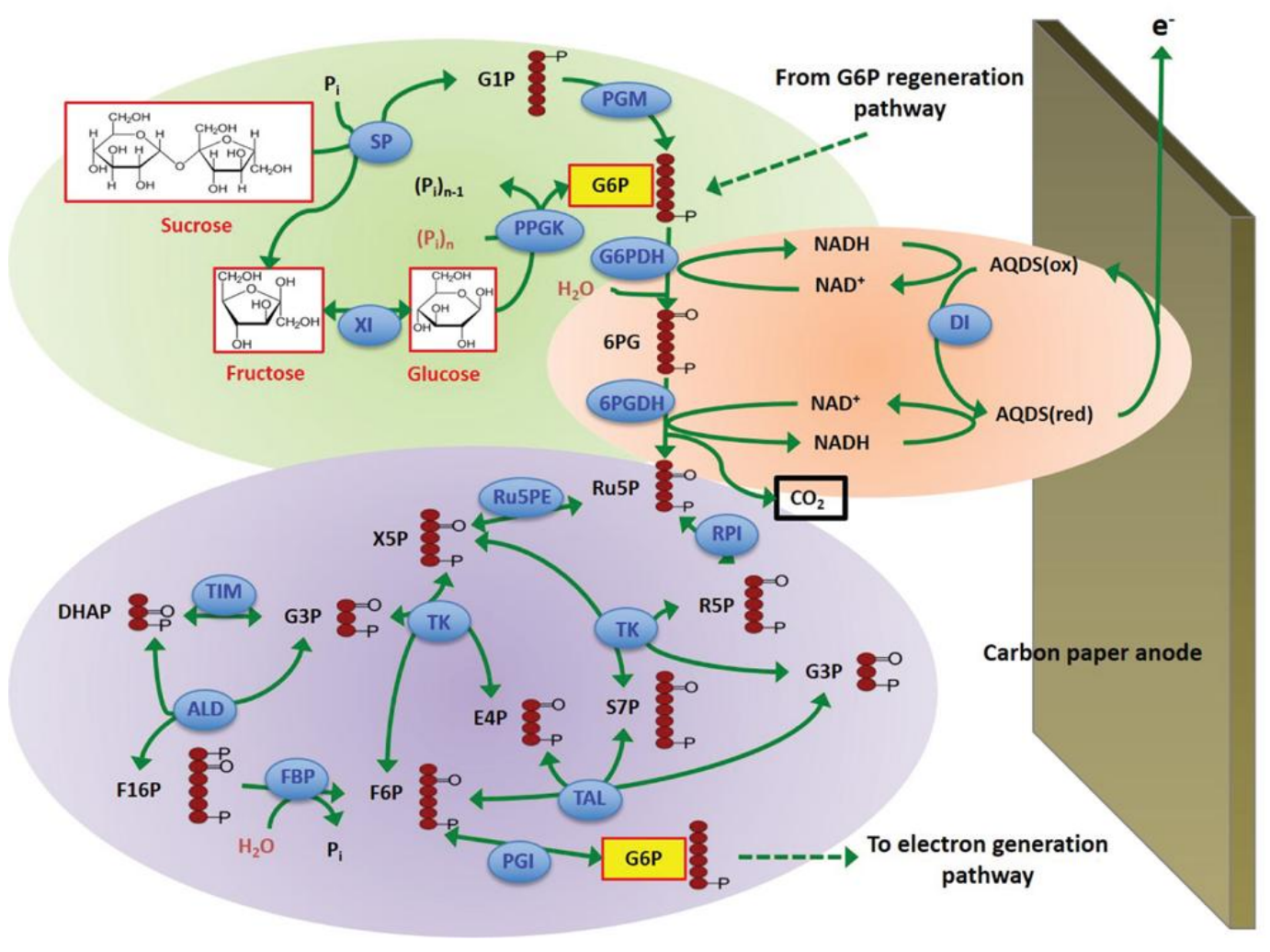


Figure 4. Schematic diagram of the in vitro 15-enzyme pathway in the anode of the EFC that can completely oxidize sucrose, fructose, and glucose at the same time. Reprinted with permission ${ }^{167}$. Copyright 2018 Elsevier.

The majority of glucose-fed EFCs are based on one oxidoreductase (i.e., GOx or NAD-dependent glucose dehydrogenase (GDH)), generating only 2 of total 24 electrons per glucose $^{52,168}$. In order to achieve more complete oxidation of glucose, Gorton et al. developed a highly efficient anode for glucose-based EFCs by combining pyranose dehydrogenase from Agaricus meleagris (AmPDH) and cellobiose dehydrogenase from Myriococcum thermophilum $(\mathrm{MtCDH})$, resulting in up to six electrons being obtained by the oxidation of one glucose molecule ${ }^{169}$. Inspired by the metabolic pathways in living cells to fully oxidize glucose, Minteer et al. ${ }^{170}$ proposed a six-enzyme system at a bioanode to oxidize glucose to $\mathrm{CO}_{2}$. It was however difficult to confirm that the complete oxidation of glucose had occurred because $\mathrm{CO}_{2}$ could be produced from intermediate reactions. Zhu et al. ${ }^{171}$ designed a novel synthetic pathway containing two NAD-dependent dehydrogenases (i.e. glucose-6-phosphate dehydrogenase and 6-phosphogluconate dehydrogenase) to perform the oxidation of glucose, generating four electrons per molecule of glucose. The same authors designed a synthetic enzymatic pathway that was comprised of 13 enzymes in an air-breathing enzymatic fuel cell to completely oxidize the glucose units of maltodextrin and generate nearly 24 electrons per glucose unit ${ }^{42}$. Three functional modules were assembled to oxidize the substrate, transfer electron, and regenerate the intermediate. First, glucose units in maltodextrin were converted to glucose 6-phosphate (G6P) by the enzymes glucan phosphorylase and phosphoglucomutase. Next, during the two-step oxidation of G6P by glucose 6-phosphoate dehydrogenase (G6PDH) and 6-phosphogluconate dehydrogenase $(6 \mathrm{PGDH}), \mathrm{NAD}^{+}$was simultaneously reduced to $\mathrm{NADH}$, which was subsequently re-oxidized by diaphorase (DI), producing two electrons per $\mathrm{NADH}$. Other enzymes were used to convert the 5-carbon intermediate to the 6-carbon G6P. The oxidation and regeneration steps were repeated six times in order to fully oxidize G6P, releasing 24 electrons. As a result, an EFC containing a 15\% (wt/v) maltodextrin solution had 
an energy-storage density of $596 \mathrm{Ah} \mathrm{kg}^{-1}$ and a faradaic efficiency of $92.3 \%$. Nevertheless, this pathway utilized polysaccharides, such as maltodextrin and starch, and cannot be applied directly to glucose. Later, based on a combination of glycolysis and the pentose phosphate pathway, an in vitro synthetic enzymatic pathway was demonstrated to generate close to the theoretically available yield of electrons from glucose. This pathway does not involve ATP, CoA, or membrane proteins. The reaction rate was enhanced after replacing several enzymatic building blocks and introducing a new enzyme, 6-phosphogluconolactonase. Using this new pathway, a high faradaic efficiency of $98.8 \%$ was obtained, with a maximum current density of $6.8 \mathrm{~mA} \mathrm{~cm}{ }^{-2}$. Similarly, an in vitro 15 -enzyme pathway that can co-utilize glucose, sucrose and fructose in EFCs was designed by incorporating the corresponding enzymes in the sugar conversion module. G6P was obtained after several sugar phosphorylation steps and then entered into the oxidation and regeneration modules as described above. The EFC achieved a faradaic efficiency of approximately $95 \%$ for these three sugars and yielded a maximum power density of $1.08 \mathrm{~mW} \mathrm{~cm}^{-2}$ (Figure 4) ${ }^{167}$. This work was the first to demonstrate the use of sugar mixtures as the fuel in EFCs and the achievement of close to the theoretically available energy density. In addition to the hexose fuels mentioned above, xylose as the second largest mono-saccharide and the most abundant pentose in plant biomass, is also a promising sugar fuel. Recently, a reconstituted bacterial pentose phosphate pathway in vitro was confirmed to generate a nearly theoretical yield of electricity from xylose in EFCs for the first time ${ }^{172}$. The complete oxidation of xylose can pave the way for the co-utilization of hexose and pentose in biomass and is a promising method for the production of bioresource-derived electricity ${ }^{172}$.

However, the use of enzyme cascades introduces a number of challenges such as increased complexity. The overall stability of an EFC is limited by the enzyme that possesses the lowest stability (e.g., as low as several hours at room temperature). The operation of EFCs can be compromised since specific enzymes have various optimal temperatures and $\mathrm{pHs}^{42}$. The amount of each immobilized enzyme on the surface of electrodes is limited with a fixed 
number of anchoring points to host the enzymes. Electrode fouling together with enzyme or cofactor degradation is an additional concern in such systems. Therefore, it makes sense to precisely localize enzymes in a sequential manner to increase the overall flux ${ }^{173}$. Enzyme complexes or in vitro metabolons, facilitating the transfer of intermediates between enzymatic steps, have been constructed as an efficient approach to accelerate cascade reactions ${ }^{174}$. Liu et al. ${ }^{175}$ synthesized an enzyme complex by covalently modifying hexokinase (HK) and G6PDH with a poly(Lys) bridge. The enzyme complex was synthetically cross-linked and able to facilitate electrostatic substrate channeling by shortening the lag time required to reach steady state. Another work reported the assembly of an ADH and AldDH enzyme cascade-based bioanode via a protein purification-free approach in a methanol biofuel cell. By using a designed DNA duplex sequence, substrate channeling between active sites of cascade enzymes was observed and the power density of the biofuel cell increased by $73 \%{ }^{176}$. In addition, enzyme complex or metabolon based EFCs have also been considered as a promising alternative to obtain significantly improvement in faradaic efficiency and stability ${ }^{177}$. To understand the mechanisms involved in such cascade reactions, differential electrochemical mass spectrometry could be used to provide relevant information on the products formed at each step ${ }^{178}$.

\section{Strategies for increasing power density in EFCs}

\subsection{Evaluation of different power output results}

The power output of EFCs is a vital criterion to determine which applications can actually be considered. In practice, the polarization curve (voltage-current profile) and the corresponding power output profile can be obtained using four methods ${ }^{179}$ : i) discharge the EFC at specified resistances by connecting the EFC to a resistor and measuring a series of currents and voltages obtained on varying the resistance; ii) potentiodynamic discharge: record the response (voltage-current plots) at a relatively slow sweep rate (typically no more than $1 \mathrm{mV} \mathrm{s}^{-1}$ ); iii) potentiostatic discharge: apply various discharge voltages and record the currents generated; iv) 
galvanostatic discharge: discharge the EFC at various currents and record the associated voltages. Accordingly, the operational stability of an EFC can also be monitored over time using these four methods. Methods i), iii) and iv) are most convenient and the most frequently used for long-term testing.

One of the issues concerned with the evaluation of the performance of EFCs is the definition of their power output. The power output is generally reported as the power density, as is widely used with batteries and other fuel cells. However, arbitrary comparison between power densities reported in the literature can be misleading in EFCs due to significant variations in the type of electrode material, method of enzyme immobilization, enzyme loading, etc.).

The majority of reports on power densities in the literature are based on the projected geometric surface area of the electrodes $(\text { Table } 1)^{11}$. However, such reported power densities do not consider the morphology of the electrode materials nor the loading of immobilized biocatalysts. In the last decade, porous nanostructured materials with high conductivities have been employed as electrodes, due to their large hierarchical porosity, and high surface areas ${ }^{92}$. The power density of EFCs based on porous nanostructured materials increased from $\mu \mathrm{W}$ $\mathrm{cm}^{-2}$ to $\mathrm{mW} \mathrm{cm}^{-2}$, calculated according to the geometric areas of the electrodes (Table 1) $43,62,180,181$. Nonetheless, these values cannot accurately represent the real power densities as due to the high porosity of the electrodes, the real surface areas are much larger than the geometric areas. Just to give one example, a compressed multi-walled carbon nanotube (MWCNT)-based bioelectrode used in a glucose EFC exhibited an electroactive area of 52 $\mathrm{cm}^{2}$ for an interfacial geometrical area of $1.3 \mathrm{~cm}^{2}$, which corresponds to $0.01 \%$ of its BET surface $\operatorname{area}^{59}$. Moreover, calculation of the power density is dependent on the method used to determine the electroactive surfaces area. The electroactive surface area based on capacitance measurements is more accurate than that obtained from voltametric response of redox molecule probes ${ }^{126}$. Additionally, in the case of an EFC utilizing a bioanode and a biocathode with different surface areas, the geometric surface area of the rate-limiting bioelectrode is 
commonly used to calculate the power density. For example, a $\mathrm{H}_{2} / \mathrm{O}_{2} \mathrm{EFC}$ requires a bilirubin oxidase (BOD)-modified cathode with an area of $6 \mathrm{~cm}^{2}$ to match the output of a $1.2 \mathrm{~cm}^{2}$ hydrogenase (Hase)-modified anode ${ }^{182}$. Therefore, balancing of the catalytic performance of a cell requires consideration of the appropriate sizes of the electrodes.

The weight or the volume of an EFC is another critical parameter in the evaluation of performance. The specific power can be determined in $\mathrm{mW} \mathrm{g}^{-1}$ or $\mathrm{mW} \mathrm{cm}^{-3}$, and is particularly suitable for three-dimension structured electrodes ${ }^{9,183}$. In terms of potential applications for portable power sources, the power density normalized to the overall weight is important, although few reports providing these values ${ }^{59}$. As a simple example, a net power of $10-20 \mathrm{~W}$ is required to power a laptop ${ }^{184}$, and a high-power-density-per-gram will help to reduce the weight. For an implantable EFC, the volumetric power density is the most important parameter (more detailed discussion is in Section 3.4). The power output of EFCs needs to be described in a manner that enables direct comparison between different systems ${ }^{59,185}$. The expression of power density in multiple forms is therefore recommended. For example, the power output of an EFC based on the naphthoquinone-mediated oxidation of glucose in a CNT 3D matrix was expressed in $\mathrm{mW} \mathrm{cm}^{-2}, \mathrm{~mW} \mathrm{~mL}^{-1}$ and $\mathrm{mW} \mathrm{g}^{-1}$ simultaneously ${ }^{41}$. The same approach was used for a membrane- and mediator-free glucose $/ \mathrm{O}_{2}$ EFC utilizing novel graphene/single-wall carbon nanotube co-gel electrodes ${ }^{186}$. Reporting the data in this format makes it feasible to compare results in an effective manner.

The number of enzyme molecules involved in the biocatalytic process is an additional key factor for the evaluation and comparison of the performance of bioelectrodes. It is thus necessary to report enzyme loadings applied on the electrode and to estimate the amount of electroactive enzymes. However, this determination is not obvious, and relies on the determination of the non-catalytic signals of enzymes obtained under non-turn-over conditions. Although this information has often been reported for $\mathrm{Lac}^{187,188}$ or $\mathrm{BOD}^{189,190}$, it has only once been reported for hydrogenase ${ }^{67}$. Low enzyme coverage can render such a determination difficult. Even when 
obtained, it is necessary to ensure that the species involved in the baseline signal appearance are identical to those involved in the catalytic measurements. This last condition is only rarely satisfied, however, leading to misleading values. The work from Mazurenko et al., should be noted where the authors rigorously followed the evolution of the non-catalytic signals related to the FeS clusters of the $A a$ Hase with the decrease of the catalytic signal for $\mathrm{H}_{2}$ oxidation, allowing the accurate determination of the amount of enzyme involved in the catalytic process ${ }^{67}$. In addition, it is essential to consider the fuel concentration when comparing the power density (Table 1).

Besides the above-mentioned factors that influence power output, an analysis of the reaction occurring in each compartment of an EFC should be carefully made. A typical bioelectrocatalytic reaction is comprised of i) mass transport of the reactant from the bulk solution to the active site on the solid surface, ii) enzymatic reaction with the reactant, iii) electron transfer between active sites of the enzyme and the electrode, and iv) diffusion of the products into the solution from the solid-liquid interface (Figure 5) ${ }^{77}$. The identification and enhancement of the current density of the limiting bioelectrode in a given system with fixed electrode geometries, either the bioanode or biocathode with the lower net catalytic current density, are crucial ${ }^{77,191}$. In the following sections, we will discuss the general strategies to increase the current density of a single bioelectrode using fast rates of $\mathrm{ET}^{80}$, in addition to mass transport issues and cell configuration design.




Figure 5. Schematic drawing of a typical electrocatalysis reaction using enzymatic or inorganic catalysts highlighting key reaction steps. Reprinted with permission ${ }^{77}$. Copyright 2018 Elsevier.

\subsection{Increasing intrinsic enzyme activities}

The catalytic response of enzymes in solution is generally characterized by the Michaelis-Menten equation $^{192}$ :

$$
\mathrm{V}=\frac{V_{\max }[\mathrm{S}]}{K_{M}+[S]}
$$

where $\mathrm{V}$ is the rate of reaction, $\mathrm{V}_{\max }$ the maximum rate of reaction, $\mathrm{K}_{\mathrm{M}}$ the Michaelis constant (the substrate concentration at which $\mathrm{V}$ is equal to $\mathrm{V}_{\max } / 2$ ). The turnover frequency, $\mathrm{k}_{\text {cat }}$ is defined as:

$$
\mathrm{k}_{\text {cat }}=\frac{V_{\max }}{[\mathrm{E}]_{\mathrm{o}}}
$$

where $[\mathrm{E}]_{\mathrm{o}}$ is the enzyme concentration. These kinetic parameters can be obtained by assaying the enzyme activity ${ }^{193}$. Enzyme activity is widely used as an indicator to compare the biocatalytic efficiency of a single enzyme using various substrates and of different enzymes for the same substrate ${ }^{194}$. The ratio $\mathrm{k}_{\text {cat }} / \mathrm{K}_{\mathrm{M}}$ is independent of the concentration of enzyme and substrate. One international unit (U) of an enzyme is defined as the amount of enzyme that catalyzes the conversion of $1 \mu \mathrm{mol}$ of reactant per min ${ }^{195}$. Accordingly, specific enzyme activity (in $\mathrm{U} \mathrm{mg}^{-1}$ or $\mathrm{U} \mathrm{g}^{-1}$ ) represents the number of units of enzyme per mg or $\mathrm{g}$ of protein. Actual enzyme activity after long-term storage of enzymes should be reported. It should be noted that these activities are defined based on their optimal conditions that may not be the same as the condition of running a specific EFC. In EFCs, attention needs to focus on the enzyme activity at the electrode interface. In many cases involving enzyme immobilization, the actual activity of immobilized enzyme may be not as high as predicted due to enzyme deactivation and mass transfer barriers. It is necessary to consider the number of enzymes per electrode or per cell in order to appropriately compare the performance of different EFC systems.

Naturally occurring enzymes may not possess sufficiently high activities that are required for EFCs. Therefore, substantial efforts have been made on enzyme engineering to improve the 
catalytic activities and rates of electron transfer ${ }^{196}$. Here, we focus on discussing the engineering on GOx, one of the most widely used enzymes in bioelectrodes, to demonstrate general strategies including direct and random site mutagenesis as well as enzyme de-glycosylation that can be used to improve the current density. Schwaneberg et al. employed directed protein evolution in Saccharomyces cerevisiae to screen libraries of mutants. They found a GOx mutant (I115V), close to the FAD centre, with 1.4-1.5 times higher activity for glucose oxidation ${ }^{197}$. A similar approach led to a double mutant GOx (T30S and I94V) that displayed an increased $k_{\text {cat }} / K_{M}{ }^{198}$. Altering expression strains is an additional route to altering the properties of enzymes. On replacing native GOx from Aspergillus niger with Penicillium pinophilum GOx at an Os-complex modified polymer "wired" bioanode, Mano et al. reported an EFC showing an increase in power density from 90 to $280 \mu \mathrm{W} \mathrm{cm}{ }^{-2}{ }^{199}$. This increase was induced by the lower $\mathrm{K}_{\mathrm{M}}(6.2 \mathrm{mM})$ of $P p \mathrm{GOx}$ compared to that of $A n \mathrm{GOx}(20 \mathrm{mM})$, resulting in a bioanode with higher catalytic current in the presence of only $5 \mathrm{mM}$ glucose. A recombinant GOx from Penicillium amagasakiense has been overexpressed in a secreted active form displaying a $\mathrm{k}_{\mathrm{ca}} / \mathrm{K}_{\mathrm{M}}$ in homogeneous glucose solution of $155 \mathrm{mM}^{-1} \mathrm{~s}^{-1}$, which was much higher than that of a AnGOx $\left(38 \mathrm{mM}^{-1} \mathrm{~s}^{-1}\right)^{200}$. Using ferrocene-methanol as a mediator, the electrocatalytic current observed towards glucose oxidation was two-fold higher with the recombinant GOx than with a native one ${ }^{200}$. De-glycosylated GOx in combination with an Os-polymer mediator showed an $18 \%$ increase in current density, which is likely a consequence of the shortened distance between the active site of the enzyme and the redox mediator, as well as improved mediator utilization due to the decreased molecular size after de-glycosylation ${ }^{201}$.

\subsection{Facilitating electron transfer}

The rate of ET plays an important role in determining the power output of many EFCs. According to Marcus's theory, the reorganization energy and the distance between the donor and the acceptor determine the rate of $\mathrm{ET}^{202}$, which decreases by an order of magnitude for every 2.3 $\AA$ increase in distance ${ }^{203}$. An upper threshold of $15 \AA$ is thus required for efficient direct electron transfer (DET) ${ }^{204}$. The structure and conformation of the enzyme may present a cofactor in close 
proximity to the electrode to maximize the rate of ET ${ }^{205}$, so that the current density of the bioelectrode can be enhanced. However, many cofactors and active sites reside inside insulating protein shells, introducing barriers to effective rates of long distance ET. In this section, we start with introducing several important equations and theories of ET. A summary of the common DET-capable enzymes along with the brief description of their structural features is followed. We then discuss strategies, such as electode surface modification for suitable enzyme orientation and enzyme engineering, to bring the enzyme cofactor closer to the electrode surface to facilitate DET for high-current-density bioelectrodes. The difference between DET and MET- based bioelectrodes will be briefly discussed, while MET will be further detailed in Section 5.1 when discussing the EFC voltage.

Protein film voltammetric techniques (PFV) can be used to characterise redox enzymes in detail $^{206,207}$. It describes the noncatalytic situation of DET-capable enzymes that are in (sub-)/monolayer configuration displaying the cofactors based well-defined voltammetry on the electrode surface. The empirical Butler-Volmer equations reveal that interfacial ET rates exponentially increase with the activation potential (driving force) ${ }^{208}$. Convenient forms of the Butler-Volmer expression describing the electrochemical rate constants for a reversible redox reaction $\left(k_{\mathrm{red}}\right.$ and $\left.k_{\mathrm{oxi}}\right)$ of a one-electron couple are:

$$
\begin{aligned}
& k_{\text {red }}=k^{0} \exp \left(-\frac{\alpha n F \eta}{R T}\right) \\
& k_{\text {oxi }}=k^{0} \exp \left(-\frac{(1-\alpha) n F \eta}{R T}\right)
\end{aligned}
$$

where $k^{0}$ is the standard first-order electrochemical rate constant, $\alpha$ is the electron-transfer coefficient, $\eta$ the activation potential, $\mathrm{R}$ the gas constant and T is the absolute temperature. $k^{0}$ can be determined by Laviron's method by plotting the voltammetric peak potential versus the logarithm of the scan rate ${ }^{209}$.

Marcus theory describes the relationship between $k^{0}$ and the tunnelling distance $d$, which is the distance between the electrode surface to the electron's entry/leaving point in the 
DET-capable enzyme ${ }^{208}$ :

$$
k^{0}(d) \infty \exp (-\beta d)
$$

where $\beta$ is a decay constant. A dispersion of tunneling distances (i.e. various enzyme orientations) leads to a dispersion of values of $k^{0}$. The mathematic model developed by Léger et al. can describe the distribution of DET-capable enzyme orientations ${ }^{208,210}$.

The surface coverage $(\Gamma)$ of (sub-)/monolayered DET-active enzymes can be evaluated from the voltammetric peak current $\left(I_{\mathrm{p}}\right)$ obtained for the oxidation/reduction of the co-factor under non-turnover conditions, which is proportional to the enzyme concentration:

$$
I_{p}=\frac{n^{2} F^{2} v A \Gamma}{4 R T}
$$

where $v$ is the scan rate of the voltammetric method and $\mathrm{A}$ is the surface area.

$\Gamma$ is equivalent to $[E]_{0}$ in eq. 3, assuming that all of the electrochemical-addressable enzymes are involved in the catalytic reaction. $k_{\text {cat }}$ can be obtained using the saturated electrocatalytic current $\left(I_{\text {cat }}{ }^{\text {sat }}\right)^{211}:$

$$
k_{c a t}=\frac{I_{c a t}{ }^{s a t}}{F n \Gamma A}
$$

The electrochemical form of the Michaelis-Menten equation is ${ }^{206}$ :

$$
I_{c a t}=\frac{n F A \Gamma k_{c a t}[S]}{[S]+K_{M}}
$$

When the bioelectrode is studied further using rotating-disc voltammetry (RDV), the limiting current $\left(I_{\mathrm{L}}\right)$ of the bioelectrocatalytic current can be described by the Koutecky-Levich approximation:

$$
\frac{1}{I_{L}}=\frac{1}{I_{\text {cat }}}+\frac{1}{I_{\text {Lev }}}+\frac{1}{I_{E}}
$$

where $I_{c a t}$ describes the intrinsic catalytic current of the enzyme, defined by eq.9; $\mathrm{I}_{\mathrm{Lev}}$ which is described by the Levich equation, is dependent on the rate of rotation and is limited by substrate diffusion between the enzyme and bulk solution ${ }^{206} ; I_{\mathrm{E}}$ is determined by the Butler-Volmer equation (eq.4 and 5) describing the rate of interfacial electron transfer between the electrode and the enzyme. For a first order reaction, 


$$
\left|I_{E}\right|=n F A \Gamma k_{E}
$$

where $k_{\mathrm{E}}$ is the heterogeneous electron transfer rate constant (i.e. either $k_{\text {red }}$ in eq.4 or $k_{\text {oxi }}$ in eq.5).

Detailed consideration of the 3D structure of a protein is essential to rationalize the electrode surface functionalization for a prefered $\mathrm{DET}^{128}$. X-ray crystallographic structures of many enzymes are available, and cryo-electron microscopy has the potential to significantly expand the number of structures and in particular of larger enzymes and enzyme complexes ${ }^{212}$. There has been much debate on considering ET via electronic relays within an enzyme subunit as a DET process $^{213}$. As an illustration, electrons generated upon $\mathrm{H}_{2}$ oxidation at the $\mathrm{NiFe}$ active site located in the large subunit of an hydrogenase travel through three FeS clusters in the small subunit of the protein ${ }^{24}$. Another relevant example is $\mathrm{CDH}$ in which the heme domain acts as the electronic relay ${ }^{132}$. In this review, ET between the electrode and the catalytic center of an enzyme via a built-in redox relay is regarded as DET (Figure 6B) ${ }^{125}$. These redox relays are present in a range of sugar oxidizing enzymes used at the bioanode. $\mathrm{CDH}$ is a flavocytochrome composed of a catalytically active flavodehydrogenase domain and a cytochrome domain acting as a built-in ET relay (Figure 6C) ${ }^{214,215}$. Another relevant example is FDH, a flavohemoprotein with three subunits ${ }^{216,217}$ : subunit I with covalently bound FAD showing a pH-dependent formal redox potential, $\mathrm{E}^{\mathrm{o}}$, of $-0.034 \mathrm{~V}$ vs. SHE at $\mathrm{pH} 5.5$ for catalytic oxidation of D-fructose; subunit II containing three heme $\mathrm{c}$ moieties with $\mathrm{E}^{\mathrm{o}}$ of $0.135,0.251$ and $0.537 \mathrm{~V}$ at $\mathrm{pH} 5.5$, the heme with the lowest $\mathrm{E}^{\mathrm{o}}$, suggested to be the exit site for ET pathway ${ }^{218}$; and subunit III, whose function is still unclear and does not carry any redox centers. Bacterial derived, hetero-oligomeric FAD-dependent GDH (FAD-GDH) consisting of a FAD based catalytic subunit, a small chaperone subunit and a multi-haem ET subunit ${ }^{219}$, is also capable of DET. A [3Fe-4S] cluster has been identified in this FAD-GDH type, acting as a ET bridge between FAD and the multi-heme cytochrome c subunit ${ }^{220}$. Quinohaemoprotein-type PQQ-dependent enzymes (e.g. $\mathrm{GDH}^{221,222}, \mathrm{ADH}^{223}$ and $\mathrm{LDH}^{224}$ ) contain PQQ prosthetic group coordinated with the apoenzyme with $\mathrm{Ca}^{2+}$ and heme-c moieties performing as ET relay ${ }^{225}$. As demonstrated by Sode et al., enzyme fusion can be employed to promote DET by introducing a cytochrome domain to the 
catalytic domain of non-quinohemoprotein-type PQQ-GDH ${ }^{226}$ and fungi-derived FAD-GDH ${ }^{227}$, suggesting a powerful strategy that can be expanded to a wide range of DET-capable fusion enzymes. The use of built-in redox relay is also the case with $\mathrm{H}_{2}$ oxidizing enzymes. [NiFe]-hydrogenases catalyzing $\mathrm{H}_{2}$ oxidation possess a [NiFe] catalytic site accompanied with Fe-S clusters distant less than $10 \AA$ to facilitate intra- then intermolecular ET between the catalytic site and either c-type or the b-type cytochromes (Figure 6D) ${ }^{88,228,229}$. On the cathodic side, the copper site $\mathrm{T} 1$ of multicopper oxidases (MCOs) is the site where the natural substrate binds. It is located near the shell of the enzyme allowing ET with the electrode surface. $\mathrm{O}_{2}$ is reduced to $\mathrm{H}_{2} \mathrm{O}$ with four electrons transferred over a short distance $(13 \AA)$ at the combined T2/T3 (binuclear) trinuclear cluster (TNC) (Figure 6A) ${ }^{11,230}$. Immobilization of the enzyme with the electronic relay facing the electrode is necessary for efficient DET to occur. Suitable electrode surfaces are crucial for appropriate enzyme orientation to ensure favorable rates of DET ${ }^{21,154,231-235}$. In this electrode-protein recognition for DET, electrostatic and hydrophobic interactions are mainly involved. In case of electrostatic driven orientation, the distribution of charges on the protein surfaces was proved to be essential for DET ${ }^{236}$. The calculation of the protein dipole moment is thus highly informative in describing favorable enzyme orientations at a charged electrode surface $\mathrm{e}^{234,237}$.

Analysis of the shape of the electrochemical signal, especially using cyclic voltammetry, allows an estimation of the distribution of orientation of the enzymes on the electrode ${ }^{208,236}$. In situ surface techniques can provide additional information about any modification in enzyme orientation or conformation after immobilization on conductive supports. Furthermore, the support material (usually made of gold) used in these techniques can simultaneously act as the electrode and makes it possible to quantify any change of the enzyme conformation and orientation under turnover as a function of applied potential, concentration of substrate, temperature, time etc. ${ }^{128,129}$ Ellipsometry can be used to examine the dielectric properties of thin films, and can provide details of the thickness of the enzyme layer. In situ ellipsometry measurements emphasized that MCOs immobilized on bare gold tend to adopt a flattened 
conformation explaining their deactivation ${ }^{238}$. The same technique showed that monolayers of $M v \mathrm{BOD}$ were formed irrespective of composition of the self-assembled-monolayer used for gold modification, although significantly different catalytic signals were recorded. Such a result is indicative of different orientations of the enzyme, which was confirmed by the electrochemical response. ${ }^{239}$ Surface plasmon resonance and quartz-crystal microbalance (QCM) can be used to quantify the total amount of enzyme immobilized on the electrode, and the variation of this quantity under turnover and as a function of the local environment ${ }^{240}$. Correlation with the electrochemical response can allow the determination of the proportion of DET-oriented enzymes $^{234}$. QCM with dissipation (QCM-D) provides additional information on the viscoelasticity of the deposited element. Using QCM-D, loss of activity of $M v \mathrm{BOD}$ was demonstrated to be related to change in enzyme hydration rather than enzyme desorption ${ }^{241}$. ATR-Fourier-transform infrared (FTIR) spectroscopy is a powerful in situ method which has been used to study changes in the secondary structures of immobilized enzymes. In the case of BODs $^{239}$, or hydrogenases ${ }^{242}$, the conservation of the intensity of the amide I and II bands, fingerprints of the polypeptide backbone, proved that the enzyme's secondary structure was maintained upon immobilization. Alternatively, surface enhanced resonance Raman (SERR) and surface enhanced IR absorption (SEIRA) are sensitive methods to monitor enzyme orientation in the immobilized state. Using SERRS on Lac immobilized on gold nanoparticles, Shleev et al. demonstrated that ET occurred via a pathway through the trinuclear cluster instead of $\mathrm{Cu} \mathrm{T}^{243}$. Heidary et al. were able to correlate enzymatic oxidation of $\mathrm{H}_{2}$ to the orientation of a hydrogenase on $\mathrm{SAMs}^{244}$. Utilizing the surface selection rules, polarization modulation-infrared reflection-adsorption spectroscopy (PM-IRRAS) was successfully used to differentiate hydrogenase orientation as a function of the hydrophobicity of the electrode ${ }^{242}$. Angles of $25^{\circ}$ and $40^{\circ}$ between the normal to the electrode surface and the $\alpha$-helix as a main component of the enzyme, were found on negatively charged and hydrophobic SAMs, respectively. Different enzyme orientation could be correlated with the rate of enzymatic oxidation of $\mathrm{H}_{2}$. A similar study was conducted on $\mathrm{Lac}^{245,246}$, demonstrating that the orientation of beta-sheet moieties controlled the rate of catalysis. Finally, in situ microscopies (atomic force microscopy (AFM), 
scanning tunneling microscopy (STM)) can be used to indicate the location of an enzyme and to also provide conformational information. Studies carried out by Ulstrup's group on nitrate reductase $^{247}$ and De Lacey's group on hydrogenases ${ }^{248}$ and ATP synthase ${ }^{249}$ are particulary relevant. For example, using in situ high-speed AFM, the dynamic motion of the dehydrogenase-cytochrome interdomain of $\mathrm{CDH}$ occurred only in the presence of the substrate, paving the way for improved understanding of the mechanism of catalysis ${ }^{250}$.

This in-depth knowledge acquired thanks to structure examination and in situ coupled techniques guide further enzyme or electrode modifications for enhanced ET rate. Different strategies have been reported in the litterature. For example, a comprehensive study was reported for the immobilization of $M v \mathrm{BOD}$ and $B p \mathrm{BOD}$ on CNTs bearing different surface charges ${ }^{236}$. The respective surface charges and dipole moments of both BODs were shown to determine the optimal electrostatic interactions between enzymes and CNT surfaces for efficient DET. Surface bearing polyaromatic hydrocarbons that mimics the natural substrates of MCOs (e.g. bilirubin for BOD ${ }^{70,251}$ ) were able to orient the enzymes with the appropriate configuration minimizing the distance towards the $\mathrm{T} 1$ site ${ }^{252-254}$. When bilirubin was adsorbed on a carbon black based electrode for BOD immobilization, the standard ET rate constant increased by a factor of 3 with a maximum current density of $5 \mathrm{~mA} \mathrm{~cm}{ }^{-270}$. Coupled with a FDH based bioanode, the fructose $/ \mathrm{O}_{2}$ EFC exhibited a considerable maximal power density $\left(\mathrm{P}_{\max }\right)$ of $2.6 \mathrm{~mW} \mathrm{~cm}{ }^{-2}$. After introduction of pyrenebutyric acid functional groups onto the electrode, the DET current density of a BOD electrode showed ca. 6-fold enhancement over randomly adsorbed system ${ }^{254}$. Some other relevant examples include, electrode surfaces functionalized with 2-carboxy-6-naphtoyl and 4-aminoaryl diazonium salt to favor DET of MvBOD and Trametes hirsuta Lac (ThLac), respectively, due to their different binding conditions surrounding $\mathrm{Cu}$ T1 pockets ${ }^{255,256}$. In particular, naphthoate-modified MWCNTs functionalized by electrografting induce favorable orientation of Magnaporthe orizae BOD (MoBOD) that can surpass MvBOD in terms of both current densities and minimal overpotentials. ${ }^{256}$ Otherwise, hydrophobic interactions have been widely used to enhance DET of Lac taking advantage of its hydrophobic pocket ${ }^{181,257,258}$, 
achieving excellent maximum current densities several $\left(\mathrm{mA} \mathrm{cm}{ }^{-2}\right)$ towards oxygen reduction at $\mathrm{pH}$ 5. For example, the efficient immobilization and orientation of Trametes versicolor Lac ( TvLac) on MWCNT electrodes using adamantane-pyrene derivative, confirmed by electrochemistry, theoretical calculations and quartz crystal microbalance experiments led to maximum current densities of $2.4 \mathrm{~mA} \mathrm{~cm}^{-2} .^{259}$

Besides electrode surface functionalization, enzyme engineering faces a growing interest for specific enzyme wiring for enhanced ET. Trametes $s p$. Lac was designed with a single pyrene group close to the $\mathrm{T} 1 \mathrm{Cu}$ site ${ }^{260}$. Specific interaction via $\pi$-stacking with $\mathrm{CNT}$ sidewalls and host-guest interaction with $\beta$-cyclodextrin, enabled a shortened ET route to the electrode, resulting in high catalytic current densities with a 4.2 fold increase over that obtained with unmodified Lac ${ }^{260}$. One very recent strategy relies on the incorporation of noncanonical amino acids into designed sites of the target enzyme ${ }^{261,262}$. The great advantage is the possibility to graft specific functions at a desired location on the protein. Click chemistry was employed to form a covalent linkage between the alkyne moiety and the electrode surface. The anchoring position on the enzyme and the linker length can be tuned to understand the mechanism of DET ${ }^{262}$. Site-directed mutagenesis was used with $\mathrm{CDH}$ to enable highly site-specific immobilization via introduced cysteine residues on the protein surface and surface-grafted maleimide groups ${ }^{263}$. Covalent binding of the variants close to the heme cofactor showed $60-80 \%$ higher DET current over the physical adsorption approach due to improved enzyme orientation ${ }^{263}$. Although not widely applied but of interest, deglycosylated enzymes permit DET due to their decreased molecular weight/size and thus shortened distances for $\mathrm{ET}^{264}$. Mano et al. reported a deglycosylated $A n G O x$ which preserved its activity with the direct redox reaction of FAD occurring at $-0.687 \mathrm{~V}$ vs. $\mathrm{SHE}^{265}$. PDH carries covalently bound FAD as the cofactor and its deglycosylated form undergoes DET on a graphite electrode ${ }^{266}$. 




C)

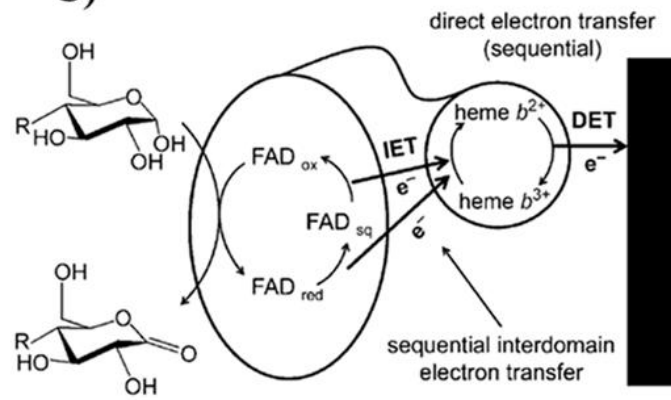

B)

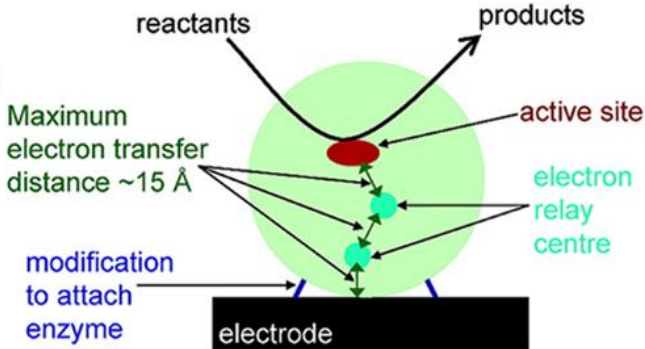

D)

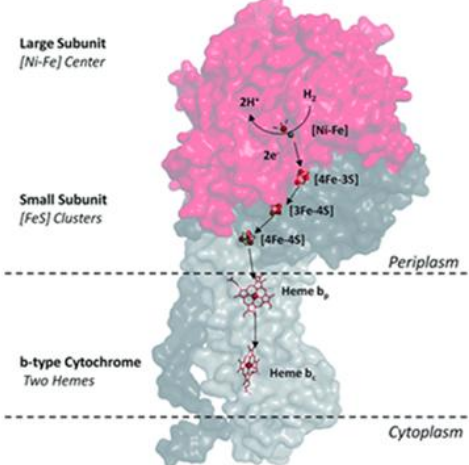

Figure 6. (A) Schematic drawing of the flow of electrons at the active site of MCOs. Reprinted with permission ${ }^{231}$. Copyright 2018 Royal Society of Chemistry. (B) Schematic drawing shows a DET capable enzyme with multi-redox centres immobilized on an electrode surface. Reprinted with permission ${ }^{7}$. Copyright 2018 American Chemical Society. (C) Schematic drawing of direct electron transfer from an aldose via $\mathrm{CDH}$ to an electrode surface. Reprinted with permission ${ }^{214}$. Copyright 2018 Wiley. (D) 3D structure of a membrane-bound [NiFe]-hydrogenase showing the electron pathway involved during physiological DET. Reprinted with permission ${ }^{88}$. Copyright 2018 Royal Society of Chemistry.

Many enzymes that have been identified for use in EFCs cannot be wired in an optimal manner, however, either because the structure is not known to a sufficiently high resolution so that the parameters for an orientation favouring DET are unknown, or because the active site is isolated from the surface by glycosylation or the presence of detergents in the case of membrane enzymes. It is noteworthy for example that native GOx cannot undergo DET at CNT or graphene based electrode ${ }^{267-269}$, as it is heavily glycosylated with an FAD group that is too deeply buried (at least $1.7 \mathrm{~nm}$ from the surface of the protein ${ }^{267}$ ) to allow direct ET. Small redox molecules can serve as exogenous mediators to shuttle the electrons via MET ${ }^{79}$. Redox mediators, which undergo reversible redox reaction, can be physiological redox partners such as cytochromes or ferredoxins, or artificial molecules including ferrocene derivatives, ferricyanide, conducting organic salts (for example, tetrathiafulvalene $(\mathrm{TTF})^{74}$ and mixed-valence viologen salt $^{270}$, etc.), quinone 
compounds, transition-metal complexes, phenothiazine and phenoxazine compounds ${ }^{205}$. To enable ET to occur, the redox potential of the mediator should be higher than that of the cofactor of the oxidizing enzyme (the opposite is true for the reducing enzyme) ${ }^{125}$. The selected mediators should also be stable in both oxidized and reduced form, access the cofactor in an efficient manner and undergo fast and reversible redox reaction on the electrode surface. Enzyme orientation is not a primary issue for MET based bioelectrode construction. For membrane-less EFCs, mediators must be co-immobilized with enzymes together on the electrode. To attain this goal, polymers bearing redox molecules on their backbones ${ }^{120,271,272}$ are widely used, however, raising performance-degrading ${ }^{273}$ and possible toxicity issues due to the potential leakage of the polymer. The possible toxicity issue from the redox polymer leakage has not been well studied and comprehensively understood. Recent reports find that some Os complexes with finely-tuned chelating ligands show comprable cytotoxicity to the clinical drugs ${ }^{274}$. Moreover, most polymer backbones are biocompatible and is unlikely to harm the body even after leakage into the body. The advantages of redox polymers in acting as $\mathrm{O}_{2}$ scavengers will be further discussed in sub-section 5.1. Meanwhile, Gross et al. recently reported the use of freely diffusing redox-active carbohydrate nanoparticles as redox mediators for homogeneous electron transfer with enzymes in solution ${ }^{275}$. This concept was illustrated via a biocathode based on 2,2'-azino-bis(3-ethylbenzothiazoline-6-sulphonic acid) (ABTS)-nanoparticles and BOD. An example of this type of EFC avoiding the need for surface immobilization at the electrode was recently illustrated with GOx and BOD electrically wired by redox organic glyconanoparticles with entrapped quinone and thiazoline redox mediators, respectively. ${ }^{276}$

The comparison between the DET and MET currents for MCOs can be used to determine the percentage of enzymes with an orientation that is unfavorable for $\mathrm{DET}^{236}$. As illustrations, the addition of free ABTS as the redox mediator increased the current density by $20 \%$ and $24 \%$ for Lac and BOD modified electrodes, respectively, emphasizing the optimal control of the proximity of enzymes' cofactor for facilitated $\mathrm{DET}^{255}$. In another study, only approximately $9 \%$ of BOD immobilized on hierarchical carbon felt modified with CNTs was electro-active ${ }^{67}$. It 
should be noted that a freely-diffusing mediator was used in these examples, with potential issues for the rate of diffusion of the mediator itself making it potentially difficult to evaluate the portion of enzyme in an orientation suitable for DET. Furthermore, examination of the DET/MET ratio demonstrated that this poor electroactive proportion was linked to unsuccessful direct enzyme wiring. On the anode side, it has been shown that Os redox polymer encapsulating CDH permitted a higher MET current density than that of DET, which can arise from a lower ratio of DET active enzymes ${ }^{277}$.

For DET-based bioelectrodes, the surface coverage of active enzymes is generally of a monolayer on the electrode. In such cases, modification of the electrode surface and the use of high-surface area electrodes is used to obtain significant currents ${ }^{100}$. In contrast, using mediators allows multilayered enzymes to be "electroactive", resulting in high current densities. A direct proof of this is the successful concept of electrostatic layer-by-layer (LBL) assembly of enzymes with redox polymers ${ }^{278-280}$. Electrostatic LBL assembly of redox enzymes and oppositely charged polyelectrolytes enables rapid ET, tuneable modification layers. However, the faradaic response of redox polymers themselves, alongside with the biocatalytic performance, does not increase linearly with increasing number of assembled layers, indicative of a limitation in the number of layers in order for the outermost layers to be electrochemically addressable, as well as limited mass transfer rates within a relatively thick polymer layer ${ }^{280}$.

A recent report combined orientation and mediation strategies to enhance the performance of a Lac-based biocathode ${ }^{281}$. A molecular wire was synthesized, which contained an enzyme-orientation site (pyrene) to be plugged into the hydrophobic pocket of Lac, an electron redox mediator (ABTS) and a pyrrole monomer to be polymerized onto electrode surface. This combined approach resulted in the highest maximum current density $\left(2.5 \mathrm{~mA} \mathrm{~cm}{ }^{-2}\right)$ in comparison to the optimal oriented $\left(1.4 \mathrm{~mA} \mathrm{~cm} \mathrm{~cm}^{-2}\right)$, mediated $\left.(2 \mathrm{~mA} \mathrm{~cm})^{-2}\right)$ and physically adsorbed approaches $\left(0.6 \mathrm{~mA} \mathrm{~cm}{ }^{-2}\right)$. Coupled with a Pt alloy/C based anode, the optimized hydroge/air fuel cell provided a $\mathrm{P}_{\max }$ of $7.9 \mathrm{~mW} \mathrm{~cm}$ (limited by the cathode). 


\subsection{Employment of nanomaterials}

Implementation of high-specific-surface-area nanomaterials including porous gold and porous carbon electrodes as enzyme supports is a widely used strategy for enhancing current density $^{88,92,103}$. Conductive materials with high surface-to-volume ratios enable high enzyme loading ${ }^{87}$. For DET enzymes, even though they may be randomly distributed in the conductive matrix, 3D nanomaterials provide the opportunity for favourable enzyme wiring for DET ${ }^{282}$. Moreover, the confinement effects of the porous electrodes are of advantage in the efficient coupling of enzymes ${ }^{282,283}$ and redox polymers modified surface ${ }^{283}$, in comparison to planar electrodes (Figure 7).

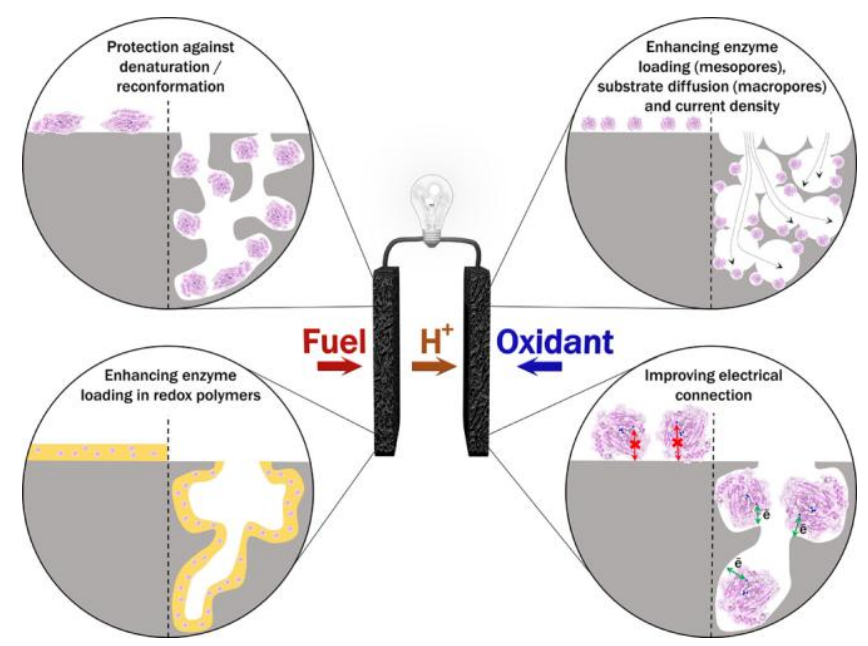

Figure 7. Schematic drawing of the advantages of using nanomaterial-based electrodes for the application of EFC. Reprinted with permission ${ }^{114}$. Copyright 2018 Elsevier.

Porous gold electrodes featuring good electrical conductivity, chemical stability and biocompatibility can be fabricated by dealloying ${ }^{282,284,285}$, Au nanoparticle (AuNP) assembly 75,286-289 , anodization ${ }^{290}$, or hard- ${ }^{291-293}$ and soft- ${ }^{294}$ template methods. Au electrodes can be easily modified with self-assembled monolayers (SAMs) of thiols ${ }^{242,295}$, diazonium grafting ${ }^{283,296,297}$ and electropolymerization ${ }^{298,299}$ for enzyme immobilization to achieve direct and mediated ET. A dealloyed porous gold (thickness: $100 \mathrm{~nm}$, pore size: $30 \mathrm{~nm}$, roughness factor: 9) based EFC utilizing electrodeposited Os-complex modified polymer with BOD and GDH, repectively, showed a $\mathrm{P}_{\max }$ of $1.3 \mu \mathrm{W} \mathrm{cm}{ }^{-2}$ in the presence of $20 \mathrm{mM}$ glucose, in contrast to $0.08 \mu \mathrm{W} \mathrm{cm}$ 
using planar $\mathrm{Au}$ electrodes ${ }^{283}$. When the same amounts of enzyme/Os-complex modified polymer were drop-cast onto electrodes ${ }^{300}$, the $\mathrm{CDH} / \mathrm{BOD}$ based EFC in $5 \mathrm{mM}$ lactose displayed 41 and $13 \mu \mathrm{W} \mathrm{cm}{ }^{-2}$ on dealloyed porous and planar gold electrodes, respectively. Such a difference arises from the larger surface area of the porous electrode. Highly ordered microporous gold electrodes assembled by AuNPs were utilized to immobilize GDH and Lac at a bioanode and biocathode respectively, and exhibited a $\mathrm{P}_{\max }$ of $178 \mu \mathrm{W} \mathrm{cm} \mathrm{cm}^{-2}$ in $30 \mathrm{mM}$ glucose, in comparison with $12.6 \mu \mathrm{W} \mathrm{cm}$ on flat electrodes $^{301}$. This increase in power density was mainly attributed to the higher enzyme loading in microporous gold. Coupling a MWCNT/GDH based bioanode with a 3D microporous gold/Lac biocathode displaying high DET current densities resulted in a $\mathrm{P}_{\max }$ of $56 \mu \mathrm{W} \mathrm{cm}$ in $^{-2} 0 \mathrm{mM}$ glucose, while $7 \mu \mathrm{W} \mathrm{cm}^{-2}$ was obtained when a planar $\mathrm{Au} / \mathrm{Lac}$ biocathode was used ${ }^{286}$. These reports highlighted the enhancement of the power density observed with porous gold electrodes. The pore size, inversely proportional to the specific surface area ${ }^{282,302}$, is an important factor for the performance of the bioelectrode. An optimal DET current for BOD was observed on porous gold electrodes with a pore size of ca. $20 \mathrm{~nm}$ which was large enough to accommodate the enzyme while providing a high surface area for sufficient enzyme loading ${ }^{283}$.

Examination of values of $\mathrm{P}_{\max }$ greater than $1 \mathrm{~mW} \mathrm{~cm}$ (Table 1) indicates that the majority of high-power-density EFCs are based on carbon nanomaterials (CNMs)-based electrodes. CNMs including buckypaper ${ }^{46,117,303}$, carbon felt ${ }^{74}$, carbon cloth, carbon black ${ }^{16}$, CNTs ${ }^{62,72,303-313}$, carbon fiber ${ }^{65,273,314-318}$, graphene ${ }^{73,93,255,319}$, porous carbon ${ }^{11,63,66,320,321}$, carbon nanodots ${ }^{322}$, and their combinations thereof ${ }^{15,35,185,323-327}$ have been widely used for the preparation of bioelectrodes. They enjoy advantages including low cost, affordable industrial scalability, wide operating potential window, chemical stability, hierarchical micro/nanostructures and flexible structures. The high specific surface area of CNMs ensures high loadings of enzymes ${ }^{67}$. For example, the modification of graphite electrode with hydrophobic carbon nanofibers (CNFs, BET surface area: $131 \mathrm{~m}^{2} \mathrm{~g}^{-1}$ ) resulted in a 1500-fold increase in the active area, so that a high current density of 4.5 $\mathrm{mA} \mathrm{cm}{ }^{-2}$ (100-fold increase) was obtained for enzymatic $\mathrm{H}_{2}$ oxidation ${ }^{328}$. Graphene coated 3D 
micropillar arrays were used to immobilize GOx and Lac, respectively, allowing an EFC with a $\mathrm{P}_{\max }$ of $136 \mu \mathrm{W} \mathrm{cm}{ }^{-2}$ in $100 \mathrm{mM}$ glucose, much higher than a bare carbon based cell $(22 \mu \mathrm{W}$ $\left.\mathrm{cm}^{-2}\right)^{329}$. As discussed previously in Section 3.3, varying the surface properties enables the electrostatic interactions between the enzyme and the electrode, resulting in the optimal preferred enzyme orientation for DET, and possible covalent attachment. CNMs are very attractive for that purpose. Versatile surface modification methods are based on diazonium salt reduction ${ }^{255,330-332}$, electropolymerization ${ }^{333,334}$ and pyrene based $\pi$-stacking interactions ${ }^{185,335-337}$. One example is that a $\mathrm{H}_{2} /$ air EFC showed a $\mathrm{P}_{\max }$ of $12 \mu \mathrm{W} \mathrm{cm}{ }^{-2}$ when the hydrogenase and BOD were randomly adsorbed on pyrolytic graphite (PG) electrode, in contrast to $119 \mu \mathrm{W} \mathrm{cm}$ using functionnalized pyrenyl MWCNTs ${ }^{254}$. By replacing PG with SWCNT-COOH, 35 and 300 fold increases in the hydrogenase-bioanode and BOD-biocathode currents, repectively, have been reported ${ }^{338}$. Accordingly, the $\mathrm{P}_{\max }$ of the resultant $\mathrm{H}_{2} / \mathrm{O}_{2} \mathrm{EFC}$ attained to a value of $300 \mu \mathrm{W} \mathrm{cm}{ }^{-2}$, much higher than that for the cell based on PG $\left.(1 \mu \mathrm{W} \mathrm{cm})^{-2}\right)^{338}$.

CNMs are versatile and can be used in various formats ${ }^{81}$, allowing the miniaturization of EFCs towards implantable applications with significant volumetric power densities. Pioneering work by Heller et al. demonstrated a glucose $/ \mathrm{O}_{2} \mathrm{EFC}$ consisting of two $7-\mu \mathrm{m}$ diameter and 2-cm long carbon fibers, which delivered a maximum power density of $137 \mu \mathrm{W} \mathrm{cm}$ (estimated to be 10 $\mu \mathrm{W} \mathrm{cm}$ in volumetric power density normalized to the whole cell size) at $37{ }^{\circ} \mathrm{C}^{339}$. The implantation of this miniaturized EFC into a grape containing more than $30 \mathrm{mM}$ glucose registered a maximum power density of $240 \mu \mathrm{W} \mathrm{cm}{ }^{-2}$ (ca. $18 \mu \mathrm{W} \mathrm{cm}$ in volumetric power density) when the cathode fiber was near the grape $\operatorname{skin}^{340}$. A glucose $/ \mathrm{O}_{2} \mathrm{EFC}$ with a needle bioanode inserting into a rabbit ear using ketjenblack as the electrode material produced a volumetric power density of ca. $42 \mu \mathrm{W} \mathrm{cm}{ }^{-3}$ (estimated by the volume of the sealing tip: 0.01 $\left.\mathrm{cm}^{-3}\right)^{341}$. A functional and implantable glucose/ $\mathrm{O}_{2} \mathrm{EFC}$ in a freely moving rat based on graphite particles electrodes was reported in 2010 , featuring a size of $0.13 \mathrm{~cm}^{-3}$ and a volumetric power

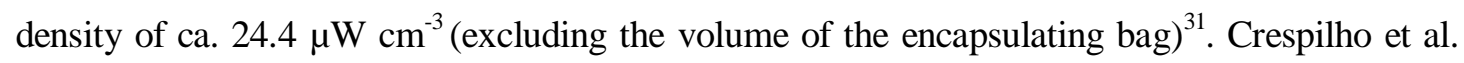
constructed a miniaturize glucose/ $\mathrm{O}_{2}$ EFC made with flexible carbon fiber microelectrodes 
(length ca. $0.05 \mathrm{~cm}$; diameter ca. $10 \mu \mathrm{m}$ ) modified with enzyme and mediator and implanted into the jugular vein of a living rat, yielding a maximum power density of $95 \mu \mathrm{W} \mathrm{cm} \mathrm{cm}^{-2342}$.

Although allowing significantly improved current densities, two main challenges of using CNMs based high-surface-area electrodes need to be addressed. Firstly, the potential toxicity of CNMs ${ }^{343}$ particularly in implantable applications, needs to be considered ${ }^{344}$. Since direct in vivo contact should be circumvented, biocompatible polymers can be used to avoid biofouling ${ }^{341}$. When used in portable devices, the possible dispersion of CNMs into the environment may cause chronic diseases with long-term exposure time ${ }^{345}$. Secondly, fuel diffusion limitations can arise at high enzyme loadings ${ }^{67,346}$. Open, hierarchical porous carbon materials with macropores for improved mass transport and mesopores with nanostructured surface for efficient enzyme-electrode communication are promising ${ }^{320,321}$. A study of the pore size effect of MgO-templated carbon on the performance of $[\mathrm{NiFe}]$-hydrogenase showed that larger pores $(150 \mathrm{~nm})$ afforded enhanced current density than small pores $(35 \mathrm{~nm})$ due to the more favourable enzyme loading in the large pores $^{347}$.

\subsection{Gas diffusion bioelectrode}

Mass transport plays a vital role in the power output of EFCs. For gaseous fuel-powered EFCs, gas diffusion bioelectrodes may overcome the substrate diffusion issue as the consumed substrate (e.g. $\mathrm{H}_{2}$ and $\mathrm{O}_{2}$ ) in the electrolyte will be compensated from the gas phase. The concentration of $\mathrm{O}_{2}$ available in aqueous solutions at room temperature is less than $1 \mathrm{mM}$, limiting power output to only a few $\mathrm{mW} \mathrm{cm}^{-2}{ }^{79}$ for EFCs based on oxygen-reducing cathodes. In the case of hydrogenase-based bioanodes, modeling of substrate diffusion showed that a thickness limited to $100 \mu \mathrm{m}$ of porous carbon material was catalytically active, mainly restricted because of fast substrate depletion in the inner layers ${ }^{67}$. Therefore, the gas diffusion bioelectrode (GDBE) is envisioned as a type of porous electrodes allowing the direct contact with gaseous substrates, eliminating the supply limitations due to the relatively low substrate solubility ${ }^{102}$. The concept of GDBE emanates from developments in conventional fuel cells. A viable GDBE consists of 
(Figure 8): ${ }^{102}$ i) a catalytic layer comprising enzymes, carbon additives for improved conductivity, binders for enhanced attachment and tuning the hydrophobicity/hydrophilicity balance and ET mediators if necessary; ii) a porous and conductive catalytic support layer such as carbon paper ${ }^{348}$, carbon cloth $^{349}$, carbon felt ${ }^{50}$ etc.; iii) a protective layer that is gas permeable and prevents leakage of the liquid electrolyte, for example Nafion ${ }^{\circledR}{ }^{350}$ and polytetrafluoroethylene (PTFE) ${ }^{351,352}$.

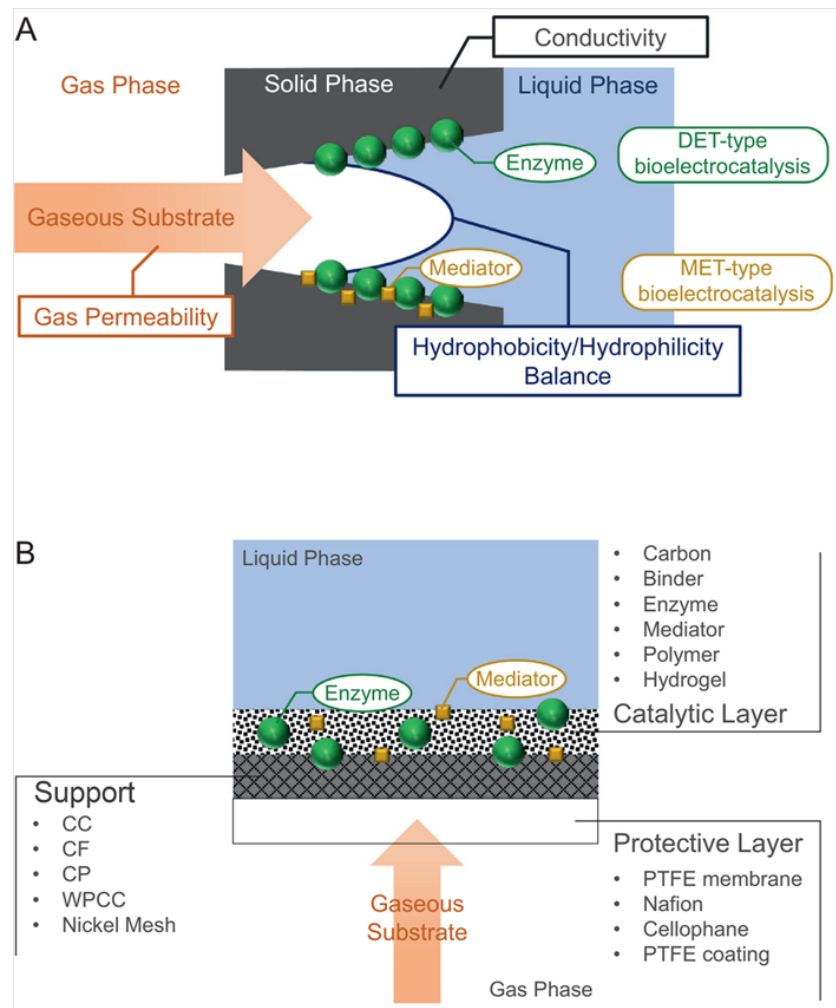

Figure 8. Schematic drawings of (A) bio-triple-phase boundary highlighting relevant properties including gas permeability, electrode conductivity and surface hydrophobicity/hydrophilicity balance; (B) structure and material candidates of a gas diffusion bioelectrode. Reprinted with permission ${ }^{102}$ with modification. Copyright 2018 Elsevier.

Initial attempts in using oxygen-reducing GDBEs were conducted by Tarasevich et al. in 2003 by immobilizing Lac at highly dispersed colloidal graphite or acetylene carbon black ${ }^{353}$. Additional reports using ferricyanide mediated $\mathrm{O}_{2}$ reduction by BOD in $2009^{52}$, a GDBE using multi-copper oxidase undergoing DET in $2009{ }^{348}$, and Lac undergoing DET $^{350}$ have been described. In pioneering work Atanassov's lab described a range of GDBEs based on $M \nu \mathrm{BOD}^{354}$ and $\mathrm{Lac}^{355-357}$ adsorbed on hydrophobized carbon black composite, and Lac on MWCNTs ${ }^{358}$. High catalytic 
current densities were reported based on GDBE not only for $\mathrm{O}_{2}$ reduction but also for other gaseous substrate enzymatic transformation such as $\mathrm{CO}_{2}$ reduction ${ }^{270}$ or $\mathrm{H}_{2}$ oxidation ${ }^{16,68,69}$. The reduction of $\mathrm{N}_{2}$ into ammonia has been possible ${ }^{153}$, which could also be developed into a gas diffusion type electrode. Recent examples demonstrate steady-state catalytic current densities at water-repellent-treated porous carbon felt/MvBOD bioelectrode as high as 24 and $32 \mathrm{~mA} \mathrm{~cm}{ }^{-2}$ using air and oxygen, respectively ${ }^{351}$. A highly gas-permeable water-proof carbon cloth/hollow MWCNTs/MvBOD displayed a DET based catalytic current density of $32 \mathrm{~mA} \mathrm{~cm}$ under atmospheric oxygen conditions ${ }^{153}$. On the anode side, the first use of GDBE for $\mathrm{H}_{2}$ oxidation was published in 2014 based on Hydrogenovibrio marinus [NiFe]-hydrogenase undergoing DET ${ }^{359}$. A $\mathrm{H}_{2} / \mathrm{O}_{2}$ EFC based on DET and dual GDBEs delivered a $\mathrm{P}_{\max }$ of $8.4 \mathrm{~mW} \mathrm{~cm}^{-2}$ at $0.7 \mathrm{~V}$ under quiescent conditions, the highest value ever reported for such device ${ }^{68}$. More recently, a dual GDBE-based $\mathrm{H}_{2} / \mathrm{O}_{2}$ EFC was constructed based on $\mathrm{O}_{2}$-sensitive hydrogenase incorporated in redox polymers and BOD directly wired to carbon cloth $^{69}$. A maximum power density of $3.6 \mathrm{~mW}$ $\mathrm{cm}^{-2}$ was obtained, one of the highest values ever reported for an EFC (Table 1) ${ }^{69}$. Beside EFC applications, GDBEs are also promising for applications in bioelectrosynthesis. The reduction of $\mathrm{CO}_{2}$ to formate using MET-type GDBEs with tungsten-containing FoDH showed a current density of $17 \mathrm{~mA} \mathrm{~cm}^{-2} 360$.

Special care has been paid to the architecture of GDBEs allowing these high catalytic performances. Bio-triple-phase boundary (BTPB) is the interface between a gaseous fuel, liquid electrolyte (buffer solution) and solid electrode, at which the catalytic reaction occurs ${ }^{102}$. The active enzymes need to be hydrated to enable catalytic activity, highlighting the importance of tuning the surface hydrophobicity/hydrophilicity properties ${ }^{102}$. The reduction of $\mathrm{O}_{2}$ at the air-breathing biocathode nicely illustrates the main issues associated with GDBEs. Biocatalytic $\mathrm{O}_{2}$ reduction involves the following steps: i) $\mathrm{O}_{2}$ from the gas phase is dissolved in the thin liquid layer around the enzymes, ii) $\mathrm{O}_{2}$ and protons diffusing from the electrolyte meet at the active site of the enzyme with production of $\mathrm{H}_{2} \mathrm{O}$, iii) excess water is repelled by the hydrophobic surface enabling the reaction to continue. An accumulation of water at the biocathode affects the final 
performance of the GDBEs over time as the flow of gases is impeded. A subtle optimization of the hydration level at the BTPB is of importance to ensure the water content is sufficiently high for efficient proton transfer in the liquid phase, and sufficiently low to ensure adequate gas permeability at the interface. In practice, the hydrophobicity/hydrophilicity balance of GDBEs can be optimized by adjusting the hydrophilic binder/hydrophobic carbon additive ratio ${ }^{68,348}$. Besides biocathode flooding, local $\mathrm{pH}$ change at BTPB also resulted in decreased operational stability of GDBEs ${ }^{102}$, which can be alleviated by using concentrated buffer solutions ${ }^{52}$. Quantitative stability performance of various GDBEs has been described in a recent review ${ }^{102}$. The observed decay in the current density can be related to changes in hydrophobicity/hydrophilicity arising from increased water flooding and decreased gas permeability. Atanassov et al. combined oxygen-reducing GDBEs with paper based lateral-flow microfluidic systems by immobilizing enzymes with carbon based inks on nitrocellulose paper ${ }^{361}$, which broadened the range of applications of EFCs, such as microfluidic paper-EFC stacks ${ }^{362-364}$.

It should be noted that GDBEs are not suitable for implantable EFCs since gaseous substrates are not substantially available in the body, but can be feasible in subcutaneous devices ${ }^{341}$. Future efforts should be devoted to optimizing the utilization levels of the immobilized enzyme, since the widely-used enzyme/binder/additive slurry casting method maybe not sufficient enough. New strategies to engineer the hydrophobicity/hydrophilicity balance of the gas permeability and porosity should be developed. For example, a gold coated porous anodic alumina (PAA), whose surface wettability can be tailored by the properties of self-assembled monolayers, has been recently used for GOx immobilization (Figure 9) ${ }^{365}$. It is found that $\mathrm{O}_{2}$ can participate to the enzymatic reaction directly from the gas phase through the channels, resulting in an 80 -fold increase compared with that of an immersion type electrode.




Figure 9. Schematic drawing of the assembly of an Au/PAA based air diffusion bioelectrode and the testing setup. The surface wettablitiy of the Au electrode is tuned by using two different types of self-assembled monolayers of thiols. Reprinted with permission ${ }^{365}$ with modification. Copyright 2018 American Chemical Society.

\subsection{Fluidic EFCs}

Fluidic configuration is one hydrodynamic strategy to overcome substrate depletion and thus increase the power density. EFCs in implanted medical devices use sugars and oxygen available under physiologically ambient conditions in soft tissue or blood vessels ${ }^{5}$. The surrounding tissue leads to additional resistance for mass transport of reactants and waste products. In contrast, EFCs implanted in blood vessels can be regarded as flow-through devices with mass transport improved in the blood stream which has a flow velocity of $1-10 \mathrm{~cm} \mathrm{~s}^{-15}$. A wide range of recent studies has used a combination of enzymatic microfluidic devices to mimic the flow conditions in blood vessels ${ }^{350,366}$. Initial attempts focused on flowing enzyme ${ }^{367-369}$ and/or cofactor $^{370}$ and/or mediator $^{371}$ solutions into micro-channels, where enzyme immobilization is essential ${ }^{350}$. Immobilization methods such as electrostatic interaction ${ }^{76,372,373}$, covalent bonding ${ }^{294,371}$ and cross-linking ${ }^{374,375}$ will be discussed further in Section 4.1.

One interesting example is a hybrid microfluidic fuel cell based on a GOx bioanode and an air-exposed $\mathrm{Pt} / \mathrm{C}$ cathode ${ }^{375}$. At a flow rate of $0.5 \mathrm{~mL} \mathrm{~h}^{-1}$ (under laminar flow), the fuel cell exhibited a decrease in $\mathrm{P}_{\max }$ from 0.6 to $0.2 \mathrm{~mW} \mathrm{~cm}^{-2}$ when tested in buffer and human blood respectively. The decrease was proposed to be a consequences of higher viscosity and the adsorption of chemical species, including protein fragments, onto the electrode surface. A single compartment lactate $/ \mathrm{O}_{2} \mathrm{EFC}$ in a $\mathrm{pH} 5.6$ buffer containing $40 \mathrm{mM}$ lactate registered a $\mathrm{P}_{\max }$ of $61.2 \mu \mathrm{W} \mathrm{cm}{ }^{-2}$, which was increased to $305 \mu \mathrm{W} \mathrm{cm}{ }^{-2}$ when operated at $3 \mathrm{~mL} \mathrm{~h}^{-1}$ in a microfluidic configuration ${ }^{376}$. The power density increased further on raising the flow rate to $9 \mathrm{~mL} \mathrm{~h}^{-1}$, but levelled off at $12 \mathrm{~mL} \mathrm{~h}^{-1}$ indicating that the response is likely due to flow-induced instabilities in the enzyme immobilization, as explained by the authors ${ }^{376}$. Porous carbon paper has been introduced into a co-laminar microfluidic ethanol/ $\mathrm{O}_{2} \mathrm{EFC}^{377}$. In this case, a low flow rate $(50 \mu \mathrm{L}$ 
$\left.\min ^{-1}\right)$ generated higher power density than that from a higher flow rate $\left(100 \mu \mathrm{L} \mathrm{min}{ }^{-1}\right)$, a result that can arise from the longer residence times in the enzyme layer at lower flow rates.

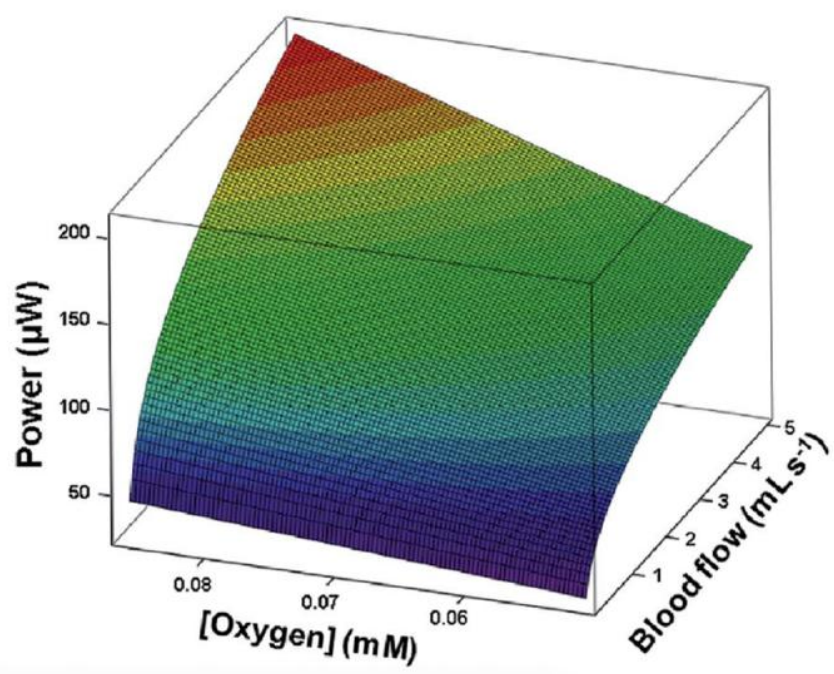

Figure 10. The calculated dependence of power output on $\mathrm{O}_{2}$ concentration and blood flow rate in a channel mimicking a human blood vessel. Reprinted with permission ${ }^{378}$. Copyright 2018 Royal Society of Chemistry.

Pankratov et al. prepared a tubular graphite electrode with inner and outer diameters of 1.00 and $3.01 \mathrm{~mm}$, respectively, sizes that resemble that of a vein ${ }^{378}$. CDH and BOD were used to modify the inner tubular surface to form a bioanode and biocathode respectively. The tube was operated ex vivo with a laminar flow of blood from a human volunteer under homeostatic conditions. Experimental data, as well as theoretical calculations, showed that the power density of such an EFC was dependent on fuel concentration and blood flow rate (Figure 10).

Detachment arising from hydrodynamic flow, however, hindered the long-term operation of the bioelectrode, highlighting the importance of robust anchoring of enzymes to electrode surfaces. Specifically, poly(ethylene glycol) diglycidyl ether (PEGDGE) cross-linked enzyme layers could be washed off by the fluid at a flow rate of $0.2 \mathrm{~mL} \mathrm{~h}^{-1} 371$. In contrast, covalently bound enzymes appeared to be much more resistant towards flow at $2 \mathrm{~mL} \mathrm{~h}^{-1} 371$. The response of a hybrid fuel cell using a GOx/MWCNTs anode and a Pt/carbon cathode was tested in human blood at a flow rate of $0.5 \mathrm{~mL} \mathrm{~h}^{-1}$, decreased by over $65 \%$ after $3 \mathrm{~h}$ of operation ${ }^{375}$. Gonzalez-Guerrero et al. 
described a pyrolyzed photoresist film (PPF) electrode based EFC employing a ferrocenium-based PEI linked GOx anode and MWCNTs/Lac cathode that displayed a decreased in power density of $50 \%$ after $24 \mathrm{~h}$ of operation at a flow rate of $4.2 \mathrm{~mL} \mathrm{~h}^{-1}$ in buffer solution ${ }^{374}$.

The Reynolds number is a measurement of degree of convective mixing of co-laminar streams ${ }^{350}$. In microfluidic channels, the Reynolds number is low, and mixing of the adjacent flowing streams is limited to a very thin interfacial width. Careful adjustment of the dimensions of these microchannels can avoid the need for a physical barrier to separate the fuel and the oxidant. Ion-exchange membranes are typically used to avoid mixing of $\mathrm{H}_{2}$ and $\mathrm{O}_{2}$ and to limit exposure to $\mathrm{O}_{2}$ inactivation of hydrogenases ${ }^{88}$. A Y-shape microfluidic channel ${ }^{369}$ with co-laminar flows of $\mathrm{H}_{2}$ and $\mathrm{O}_{2}$ enriched solution could eliminate the need for separation membranes, while ensuring rapid rates of transport of the substrates.

Other hydrodynamic environments introduced by magnetic stirring ${ }^{273}$, electrode rotation ${ }^{230,287}$ and flow cell ${ }^{33,294}$ are widely used to compensate for diffusion limitations in order to increase the power output of a EFC. However, the additional power, sometimes even greater than that generated by the EFC itself, is usually required, making these stirred and rotated EFCs impractical.

\subsection{Combined EFCs/(super)capacitor devices}

The energy generated in EFCs can be stored in energy storage devices in a 'stationary' mode. Rechargeable batteries and supercapacitors are the most widely studied energy storage devices, and can store and release electrical energy by electrochemical reactions ${ }^{379}$. Supercapacitors, also known as electrochemical capacitors, utilize the electrical double layer capacitance (EDLC) via ion adsorption or pseudocapacitance attained during reversible faradaic reactions ${ }^{379}$. Unlike EFCs suffering from low power density and stability, supercapacitors possess high specific power density and long lifetime. A range of reports have combined EFCs with (super)capacitors ${ }^{140}$. 
Initial attempts focussed on the external connection of EFCs with capacitors or supercapacitors. The coupled capacitor element accumulates the charge generated by the EFCs in the circuit, which can be released by output pulses with much higher power densities than that possible by EFCs themselves. Skunik-Nuckowska et al. used MWCNT based supercapacitors in parallel as complementary power units coupled with a Lac cathode and zinc anode based biobattery. The biobattery alone delivered a power output of $1.3 \mu \mathrm{W}$, in comparison to $8.5 \mathrm{~mW}$ from the biobattery/supercapacitor hybrid system ${ }^{380}$. Sode et al. developed the concept of a "BioCapacitor" by integrating an EFC with a charge pump/capacitor combination (Figure 11) ${ }^{139,381}$. The capacitor was gradually charged and then discharged to power the electric device ${ }^{139}$. The high-power levels generated resulted in very short discharge intervals. A similar setup was used to design a wireless sensor fed by a $\mathrm{H}_{2} / \mathrm{O}_{2} \mathrm{EFC}{ }^{382}$. The concentration of fuel determines the rate of charge/discharge of the biocapacitor, which enables a self-powered biosensor to determine the concentration of the fuel via the rate of charge/discharge of the capacitor ${ }^{139}$. Liu et al. coupled two series-connected glucose/ $\mathrm{O}_{2}$ EFCs consisting of a buckypaper/MWCNT/FAD-GDH bioanode and a buckypaper/MWCNT/Lac biocathode with a flexible all-solid-state supercapacitor ${ }^{303}$. The self-charged system achieved a $\mathrm{P}_{\max }$ of $608 \mu \mathrm{W} \mathrm{cm}{ }^{-2}$ when the capacitor was discharged, $90 \%$ higher than the value for series-connected EFCs.



Figure 11. Schematic drawing of the principle of a biocapacitor. A charge pump can scale up the voltage of the EFC and a capacitor is used to store the electrical energy. The stored electric 
energy can be discharged from the capacitor to activate a device (e.g. a LED bulb) when the capacitor voltage attains to the set value. Reprinted with permission ${ }^{139}$. Copyright 2018 Elsevier.

Closer examination of the configuration of EFCs and supercapacitors indicate that i) two electrodes hosting active materials (namely the anode and cathode) connected to the external circuit; ii) an electrolyte solution with conducting ions; iii) a separator if necessary to prevent possible short-circuit. Carbon nanomaterials that are widely used for the preparation of bioelectrodes have significantly high EDLC. Agnès et al. developed an EFC consisting of a compressed porous CNT matrix modified GOx bioanode and a Lac based biocathode, delivering $3 \mathrm{~mA}$ and $2 \mathrm{~mW}$ pulses with a short duration of $10 \mathrm{~ms}$ per $10 \mathrm{~s}$ for 5 days in the presence of glucose and $\mathrm{O}_{2}{ }^{383}$. In this case, the electricity generated by the EFC was stored continuously in the EDLC of CNTs. In parallel, Pankratov et al. reported a hybrid device based on flat graphite foil electrodes, with one face bearing an EFC using an AuNPs-CDH bioanode and an AuNPs-BOD biocathode, and the other face configured with capacitive materials (CNT/polyaniline) ${ }^{384}$. It displayed an initial power output of $1.2 \mathrm{~mW} \mathrm{~cm}^{-2}$ at a voltage of $0.38 \mathrm{~V}$ which is 170 times higher than that of the EFBC alone.

It should be clarified that a biodevice simultaneously functionning as an EFC and a supercapacitor is different from systems based on the external connection of an EFC to a (super)capacitor. The former can be termed a hybrid EFC/supercapacitor device, which can be prepared from bifunctional electrodes ${ }^{140,385}$. The integration of such systems enables miniaturization. The self-powered capacitor functions in a sequence of charging and discharging ${ }^{283,386}$. Under charging conditions, the cell is at open-circuit, and the open-circuit potential (OCP) between the two electrodes gradually increases to the open-circuit voltage (OCV) of the EFC, as a result of the higher potential on the biocathode catalyzing oxygen reduction and the lower potential at the bioanode catalyzing the oxidation of fuels. Such a potential difference drives the polarization of the capacitive biocathode and bioanode. In other words, the capacitive cell is electrostatically charged by the biocatalytically induced potential difference. In the discharge step, the accumulated charge can be released at a fixed resistance ${ }^{384}$ or current 
density ${ }^{283}$. Based on such a methodology, Kizling et al. reported a fructose $/ \mathrm{O}_{2}$ EFC/supercapacitor hybrid device composed of a cellulose/polypyrrole/FDH bioanode $e^{387,388}$ and a naphthylated CNTs/Lac biocathode ${ }^{388}$. Three biodevices in a series could generate pulses for 45 $\mathrm{s}$ with potentials above $1 \mathrm{~V}$. Villarrubia et al. prepared a buckypaper based glucose $/ \mathrm{O}_{2}$ EFC/supercapacitor hybrid device ${ }^{364}$, which could be self-charged and discharged by a range of current densities as high as $4 \mathrm{~mA} \mathrm{~cm}{ }^{-2}$ for $0.01 \mathrm{~s}$ with a $\mathrm{P}_{\max }$ of $0.87 \mathrm{~mW} \mathrm{~cm}$ (absolute $^{-2}$ maximum power: $10.6 \mathrm{~mW}), 10$ times higher than that of the EFC itself.

In addition to carbon nanomaterials with high EDLC, the pseudocapacitance behavior of redox polymers has also been examined. Knoche et al. prepared a hybrid device consisting of a carbon felt/MWCNT/dimethylferrocene-modified linear poly(ethylenimine) $\left(\mathrm{FcMe}_{2}\right.$-LPEI)/FAD-GDH bioanode and a biocathode based on a carbon felt/anthracene terminated MWCNT/BOD ${ }^{389}$. The $\mathrm{FcMe}_{2}$-LPEI redox polymer served as mediator, enzyme immobilization matrix and as a supercapacitor whose pseudo-capacitance increased with polymer loading. The device generated $1 \mathrm{~mA}$ pulses for $1 \mathrm{~s}$ with a power output of $1 \mathrm{~mW}$ energy. Pankratov et al. developed a capacitive EFC using the same Os-complex modified polymer on a GDH anode and a BOD cathode ${ }^{378}$. The capacitance of the polymer was used for energy storage with an OCP up to $0.45 \mathrm{~V}$, which could be discharged with 8 -fold higher power output than that obtained in steady state ${ }^{378}$. Xiao et al. doped an Os-complex modified polymer based FAD-GDH bioanode and a BOD cathode with poly(3,4-ethylenedioxythiophene) (PEDOT) that showed enhanced capacitance ${ }^{283}$. The hybrid device was charged by the internal glucose $/ \mathrm{O}_{2} \mathrm{EFC}$ and discharged as a supercapacitor at various current densities up to $2 \mathrm{~mA} \mathrm{~cm}{ }^{-2}$ registering a $\mathrm{P}_{\max }$ of $609 \mu \mathrm{W} \mathrm{cm}{ }^{-2}$, a 468 -fold increase when compared to that from the EFC itself. Connection of three devices in series produced 10 $\mu \mathrm{A}$ for $0.5 \mathrm{~ms}$ at a frequency of $0.2 \mathrm{~Hz}$, mimicking the power requirement of a cardiac pacemaker. Interestingly, Alsaoub et al. demonstrated that the pseudo-capacitance of Os-complex modified polymers can be used in a so-called "biosupercapacitor" which can be discharged ${ }^{390}$. However, such a biodevice, comprised of a high-potential Os complex modified 
polymer for glucose oxidation and a low one for oxygen reduction, is not an EFC as it cannot provide a usable OCV.

To briefly summarize this section, unconventional configurations of EFCs enable additional functionalities. However, as already discussed, the output voltage is still limited by the OCV of the EFC. The arbitrary combination of EFC and supercapacitor may be problematic, as shared electrodes configuration may reduce the efficiency of EFCs due to diffusion limitation. Insulating biomolecules are unlikely to be very beneficial for high-performance supercapacitors, although there are some reports indicating that proteins could contribute additional capacitance ${ }^{391}$. Additional research is needed to understand the mechanism of operation ${ }^{392,393}$ and to develop practical applications ${ }^{392}$ of hybrid devices.

\section{Strategies for improving stability in EFCs}

\subsection{Enzyme immobilization approaches}

The primary consideration of immobilization upon the bioelectrode's operational stability is to avoid enzyme detachment and other co-factors from the electrode ${ }^{76}$. Once immobilized, enzymes usually exhibit extended lifetime compared to those in solution ${ }^{394}$. Rigidification of the structure of the enzyme can enhance enzyme stability ${ }^{395}$. In EFCs, enzymes can be immobilized onto solid electrode surfaces by a range of approaches that include physical adsorption, covalent binding, entrapment and cross-linking ${ }^{6,40,90,96,396}$ (Figure 12). 


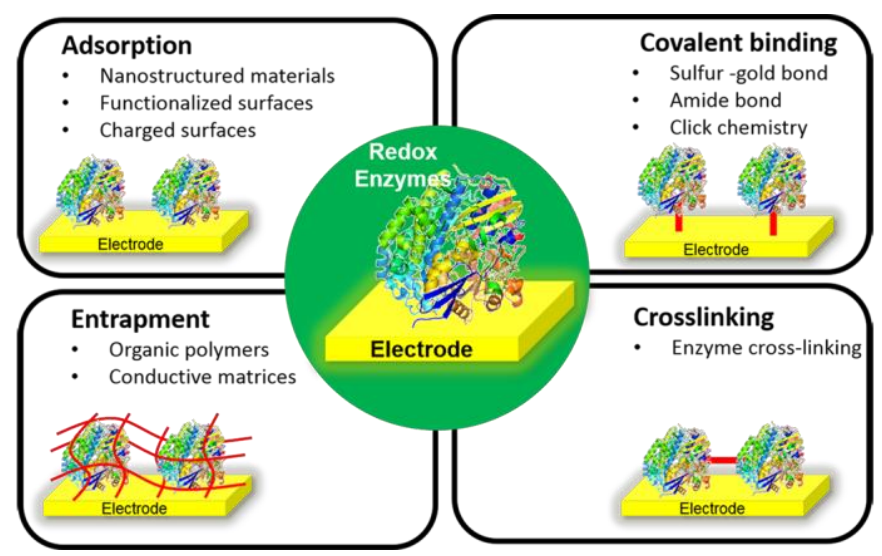

Figure 12. Scheme of various enzyme immobilization methods for bioelectrode fabrication.

Physical adsorption is generally considered as the simplest and mildest technique for enzyme immobilization, whereby enzymes are adsorbed directly onto electrode surfaces. However, enzymes physically adsorbed onto bare metal electrode surfaces often suffer from reduced operating lifetimes. ${ }^{238}$ The self-assembly of a monolayer of thiols on the electrode surface may protect the enzymes from denaturation caused by metal-enzyme interactions ${ }^{397}$ or at the electrode solution interface ${ }^{398}$. On the contrary, in comparison with planar electrodes, bioelectrodes fabricated by adsorbing enzymes at nanomaterials often exhibit not only improved electrocatalytic activity but also improved operational stability. ${ }^{399-401}$ The improved activity and stability of nano-structure-based bioelectrode was ascribed to the 3D structure of the electrode, providing a mild microenvironment to effectively avoid enzyme desorption and denaturation. ${ }^{402}$ A glucose $/ \mathrm{O}_{2} \mathrm{EFC}$ prepared by the physical adsorption of GOx and BOD onto hierarchical metal-oxide mesoporous electrodes showed only $10 \%$ loss in voltage output after 30-h continuous operation. ${ }^{403}$ Furthermore, the effect of pore size of porous electrodes on the stability of bioelectrode was investigated $231,347,404,405$. AaHase adsorbed onto MgO-templated carbon (MgOC) with pore size of $35 \mathrm{~nm}$ (larger than the size of $A a$ Hase $c a .17 .7 \pm 1.3 \mathrm{~nm}$ ), exhibited a half-time of $81 \mathrm{~h}$ at $50{ }^{\circ} \mathrm{C}$, much longer than that of $\mathrm{AaHase}$ in solution $(7 \mathrm{~h})\left(\right.$ Figure 13). ${ }^{347} \mathrm{~A}$ dialysis membrane (cut-off-molecular-weight of $12-14 \mathrm{kDa}$ ) was placed on the electrodes to prevent enzyme leakage to the solution during the measurement. The enhanced stability was ascribed to interactions between the pore materials and enzymes which decreased the conformational flexibility of the enzymes. ${ }^{347}$ 

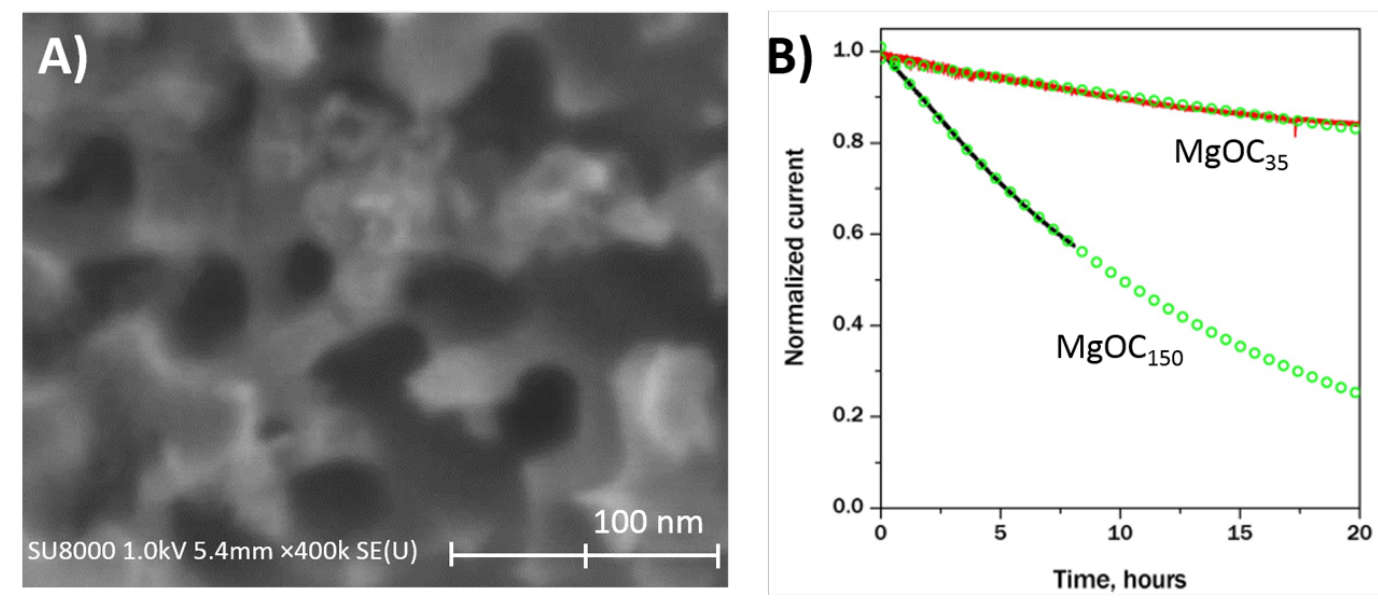

Figure 13. A) Field emission scanning electron microscopic images of $\mathrm{MgOC}_{35}$. B) Normalized response of $\mathrm{AaHase-modified} \mathrm{(red} \mathrm{line)} \mathrm{MgOC}_{35}$ and (black line) $\mathrm{MgOC}_{150}$ at $50{ }^{\circ} \mathrm{C}$ in $\mathrm{pH} 7$ phospahte buffer $(0.1 \mathrm{M}) . \mathrm{MgOC}_{35}$ and $\mathrm{MgOC}_{150}$ represent a $\mathrm{MgO}$-tempted carbon with pore size (diameter) of ca. 35 and ca. $150 \mathrm{~nm}$ repectively. Reprinted with permission ${ }^{347}$ with modification, Copyright 2018 Elsevier.

The surface properties of electrodes, such as hydrophobicity, surface charge or functionalized groups, can influence the stability of bioelectrodes ${ }^{406} . T v$ Lac adsorbed onto 1-pyrenebutyric acid adamantyl amide functionalized MWCNTs (ADA-MWCNTs) remained 66\% of the initial bioelectrocatalytic activity after 1 month $^{259}$. However, $T \nu$ Lac on pristine MWCNT exihibited rapid decrease in catalytic currents to less than 5\% of the initial value after 20 days (Figure 14). In this case, modifier with polycyclic aromatic structure can bind to the active center pocket of the enzymes, diminishing the desorption of the enzyme and reducing the conformational changes of enzymes ${ }^{259,407,70,408}$. A thermostable $\mathrm{H}_{2} / \mathrm{O}_{2}$ EFC was assembled with an AaHase-based bioanode and a $B p B O D-b a s e d$ biocathode, which were fabricated by physically adsorbing $A a \mathrm{Hase}$ or $\mathrm{BpBOD}$ onto 1-pyrenemethylamine hydrochloride functionalized CNT electrodes, retained $95 \%$ of the initial power after $17 \mathrm{~h}$ continuous operation ${ }^{67}$. These reports suggest that functionalized surfaces of electrodes are likely to improve the stability of bioelectrodes by enhancing intermolecular interaction between enzymes and electrodes as well as reducing the conformational disruption ${ }^{70,181,257,409-411}$. Conformational changes of the enzymes during operation of EFCs is an area that needs investigation. 



Figure 14. A) Schematic of $T \nu \mathrm{Lac}$ oriented adsorption on 1-pyrenebutyric acid adamantyl amide functionalized MWCNTs (ADA-MWCNT). B) Long-term stability of TvLAC at pristine MWCNTs (black) and ADA-MWCNTs (blue). Measurements were carried out by performing a daily $1 \mathrm{~h}$ discharge at $0.2 \mathrm{~V}$ in stirred oxygen-saturated $\mathrm{Mc}$ Ilvaine buffer $\mathrm{pH}$ 5. Reprinted with permission ${ }^{259}$ with modification. Copyright 2016 American Chemical Society.

Covalent binding is a typical and effective enzyme immobilization method. Peripheral amino or carboxylate groups on enzymes are feasible positions for covalent linkage. One common approach to anchor enzymes onto electrode surfaces is usually based on the formation of amide bonds between carboxylic groups and amino groups ${ }^{230,234,253,286,412-414}$, which are typically mediated by carboxylate activating reagents such as 1-ethyl-3(3-dimethylaminopropyl)carbodiimide (EDC). With the aid of $\mathrm{EDC}$, the $M v \mathrm{BOD}$ covalently bound to 6-mercaptohexanoic acid functionalized Au electrode retained more than $80 \%$ of the intial current after $4000 \mathrm{~s}$ continued measurement, while only $20 \%$ of the intial current was remained at the $M v \mathrm{BOD}$ physically adsorbed 6-mercaptohexanoic acid functionalized $\mathrm{Au}$ electrode (without EDC in this case). ${ }^{234}$ (Figure 15A, B) The covalent binding between enzymes and electrodes is expected, for example, to prevent the enzyme orientation changes, which, however, affect the interfacial ET and then the lifetime of bioelectrodes. Besides, "click chemistry" is a convenient method to covalently bind redox enzymes to electrode surfaces. ${ }^{263} \mathrm{~A}$ thiol-maleimide click reaction between $M o B O D$ variant S362C and maleimide-functionalized MWCNT was employed to construct a stable $\mathrm{O}_{2}$ bioanode, which retained ca. $95 \%$ of the initial current after 3 days storage, with ca. $30 \%$ of electrocatalytic current retained after 3 hours storage 
for an electrode prepared by simply physical adsorption ${ }^{415}$. (Figure 15 C, D) Compared to physical adsorption, covalent binding provides stronger degrees of interaction between the enzymes and the electrode surface, leading to higher stability of the bioelectrodes ${ }^{416,417}$. However, it should be noted that the enzyme activity can decrease to certain extent upon immobilization $416,418,419$


Figure 15. A) Scheme of electrode modification with $M v \mathrm{BOD}$ via an amide bond. B) Chronoamperometry of $M v \mathrm{BOD}$ at 6-mercaptohexanoic acid functionalized Au electrode with (blue) and without (black) the aid of EDC. C) Scheme of electrode modification with MoBOD variant S362C via maleimide/thiol bond; D) Maximum electrocatalytic activities of the maleimidemodified GC/MWCNT electrodes covalently modified with MoBOD variant S362C (black line) and adsorbed wild type BOD (red line) as a function of the length of storage. Reprinted with permission ${ }^{234,415}$ with modification. Copyright 2016, 2019 American Chemistry Society.

It is a useful method to entrap enzymes into polymer matrices or inorganic frameworks on the electrode surface for enzyme immobilization, which can reduce the amount of leaching while 
avoiding denaturation and conformational changes to the enzyme. Sol-gel based methods have been frequently reported to entrap enzymes with high activity and stability ${ }^{420-424}$. Entrapping redox enzymes into membrane-like matrices, such as lipid, agarose and DNA-hydrogel, which can mimic the natural environment of enzymes, retains enzymes in functionally active forms ${ }^{425-428}$. However, such matrices usually suffer from poor conductivity, and cannot be used directly for bioelectrocatalysis. As a result, materials with high conductivity such as CNTs, and redox mediators such as ferricyanide and ABTS, are usually co-encapsulated. A stable current response was obtained at a bioelectrode fabricated by coating a mixture of chitosan, Lac and MWCNT for over 60 days' continuous measurement, with more than $70 \%$ of the initial current retained upon storage for six months. ${ }^{429}$

On the other hand, by directly using conductive materials including CNTs as matrices for encapsulation of enzymes, stable bioelectrodes with high electroactivity can be realized..$^{59,71,430,431}$ Cosnier and coworkers reproted a glucose/ $\mathrm{O}_{2} \mathrm{EFC}$ by combining a GOx-based bioanode and Lac-based biocathode, prepared by mixing GOx or Lac with CNT, that showed only a slight (4\%) decrease in the maximum power density after 30 days storage in buffer solution (Figure 16) ${ }^{59}$. Recently, as novel matrices, metal organic frameworks (MOFs) have been reported to entrap enzymes with long lifetimes ${ }^{432-436}$. A stable $\mathrm{O}_{2}$ reduction enzyme electrode was prepared by mixing Lac with ABTS, mesoporous Fe(III) trimesate nanoparticle and carbon blacks $^{433}$. With improved operating lifetimes, MOFs-based enzyme-electrodes are potentially of interest in the construction of EFCs. 


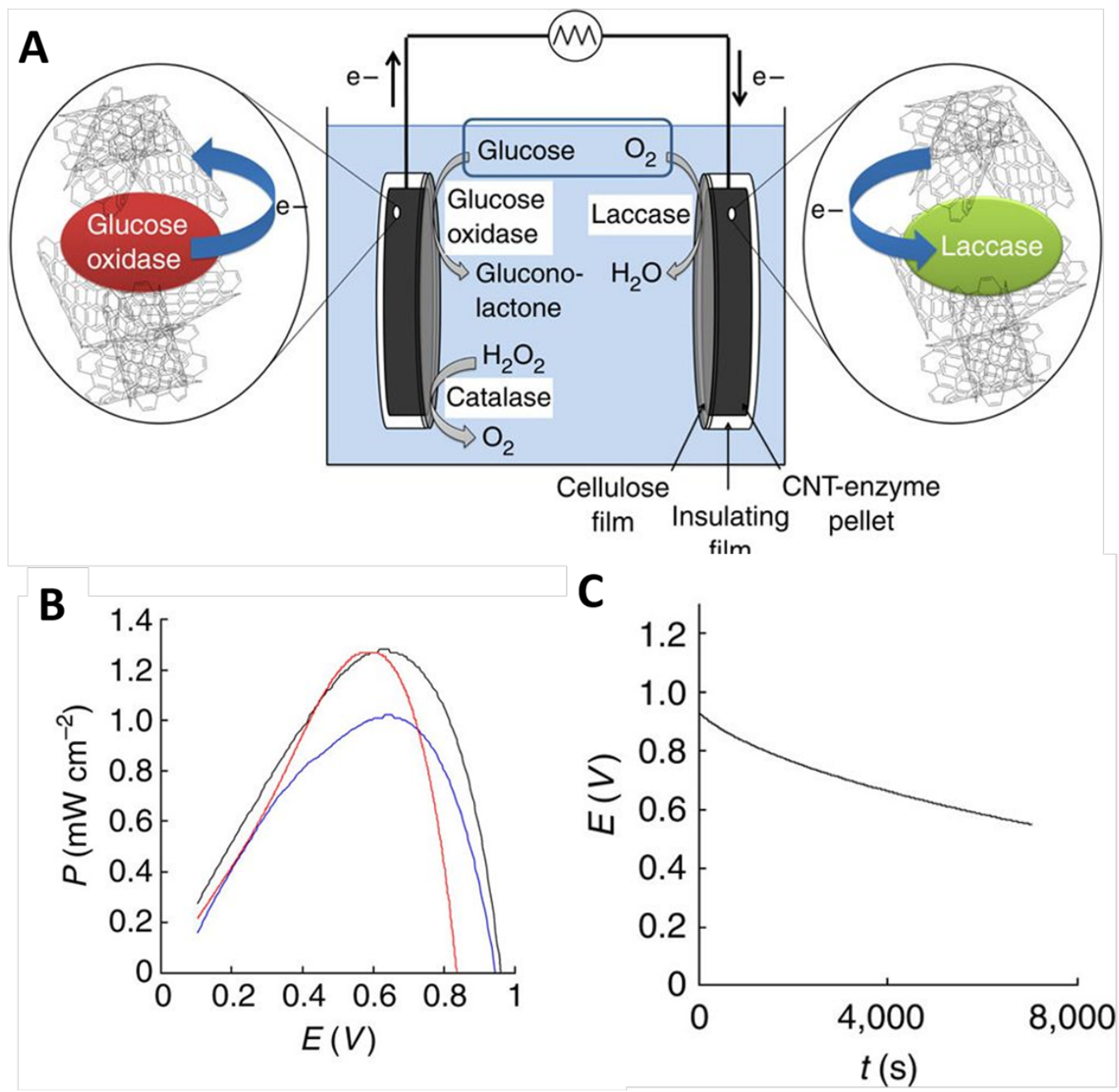

Figure 16. A) Schematic of a glucose/ $\mathrm{O}_{2}$ EFC by entrapping GOx and Lac into CNTs covered with cellulose film. B) Dependence of power density on operating voltage in $0.05 \mathrm{~mol} \mathrm{~L}^{-1}$ glucose solution before (black) and after one month (red). Blue curve: dependence of power density on operating voltage in $5 \times 10^{-3} \mathrm{~mol} \mathrm{~L}^{-1}$ glucose.C) Dependence of voltage on time for continuous discharge under $200 \mu \mathrm{A} \mathrm{cm}^{-2}$ in $0.05 \mathrm{~mol} \mathrm{~L}^{-1}$ glucose solution. Experiments carried out in air-saturated phosphate buffer $0.1 \mathrm{~mol} \mathrm{~L}^{-1}, \mathrm{pH} 7.0$ (bioanode: catalase/GOx ratio 1:1, biocathode: $20 \%$ laccase). Reprinted with permission ${ }^{59}$. Copyright 2011 Nature Publishing Group.

Cross-linking is a simple and effective method to immobilize enzymes on electrode. High stability should be expected because the process is based on bi- or multi-functional reagent ligands, forming rigid enzymatic aggregation, reducing leaching of the enzyme and improving the stability. ${ }^{437,438}$ A stable peroxidase layer at ketjen black surface for DET-type bioelectrocatalysis has been reported by using glutaraldehyde as a cross-linker. In the absence of 
glutaraldehyde, however, the catalytic current decreased with time and was lower than that in the presence of glutaraldehyde. ${ }^{439}$ On the other hand, considering that the enzyme and cross-linking agents can form covalent bonds, the stability of the resultant enzymatic bioelectrodes can be improved $^{288,440-442}$. With the aid of PEGDGE, a MET-type EFC using covalently anchored GOx and BOD, respectively at amino group-derivatized bioanode and biocathode, retained $70 \%$ of the initial maximum power after $24 \mathrm{~h}$, while just $10 \%$ retention was observed for the EFC based on underivatized graphite ${ }^{442}$. Furthermore, to avoid uncontrollable cross-linking reaction, Schuhmann's group proposed an electrochemically induced cross-linking strategy to improve the operational stability of enzymatic bioelectrodes by locally entrapping enzymes into polymers on electrode surfaces ${ }^{43-446}$. With high stability, reusability as well as high volumetric activities, cross-linking represents an alternative to conventional immobilization approaches on solid surface. ${ }^{447}$ However, a heterogeneous enzyme orientation distribution may induce slower ET rates specially in case of the use of cross-linkers.

\subsection{Tuning enzyme properties}

\subsubsection{Employing extremophile enzymes}

Biodiversity offers a large variety of microorganisms growing in extreme environments such as extreme $\mathrm{pH}^{18}$, high salinity ${ }^{448}$ or extreme temperatures. Exploiting enzymes from these microorganisms is still in its enfancy in the domain of EFCs, but may enhance the capabilities of EFCs to operate under broader operating conditions, while also potentially improving the stability of the enzymes. The discovery and use of thermostable enzymes from thermophilic microorganisms are of great importance to increase enzyme stability and potentially decrease enzyme production cost by simplifying enzyme purification process and extending enzyme duration in the area of biomanufacturing ${ }^{449}$. In EFCs, the same idea has been adopted to maintain the stability of the enzymatic catalysts and extend the lifetime of the fuel cells. Ohsaka et al. demonstrated the successful application of thermophilic GDH and laccase in EFCs that could be operated at elevated temperatures $\left(76{ }^{\circ} \mathrm{C}\right)^{450}$. Lojou et al. constructed an EFC employing a 
hyperthermophilic $\mathrm{O}_{2}$-tolerant hydrogenase and a thermostable BOD with a maximum power output of $2.3 \mathrm{~mW}$ at $50{ }^{\circ} \mathrm{C}$. The device delivered $15.8 \mathrm{~mW}$ h of of power after $17 \mathrm{~h}$ of continuous operation $^{67}$. An efficient membraneless air-breathing/ $\mathrm{H}_{2}$ EFC was assembled by Cosnier's group relying on the same thermostable enzymes ${ }^{65,349}$. Comparison of two bioanodes constructed with the hyperthermophilic Aquifex aeolicus and the mesophilic Ralstonia eutropha hydrogenases clearly demonstrated the higher stability of the former not only at elevated temperature but also at room temperature ${ }^{335}$. Another $\mathrm{NiFe}$ hydrogenase from the hyperthermophilic bacterium Pyroccocus furiosus showed a remaining activity at $80^{\circ} \mathrm{C}$ upon exposure to $\mathrm{O}_{2}$, highlighting combined resistances of these extremophile enzymes ${ }^{451}$. A Sulfolobus kodaii alcohol dehydrogenase was investigated for use as a biocatalyst for electrochemical applications by Ohno et al. The constructed bioanode can maintain a high current density at even $80{ }^{\circ} \mathrm{C}$, with a 12 -fold increase over that at room temperature ${ }^{17}$. Recently, Zhang's group developed several synthetic enzymatic pathway-catalyzed EFCs comprised of multiple thermostable enzymes cloned from various thermophiles and capable of deeply or completely oxidizing sugar fuels. Such EFCs could be operated at $50{ }^{\circ} \mathrm{C}$ and exhibit an 8 -fold increase in power density compared to those based on mesophilic enzymes ${ }^{171,452}$.

\subsubsection{Protein engineering for better stability}

As discussed in previous sections, protein engineering approaches are mainly used to improve rates of the ET between enzymes and electrodes ${ }^{453}$, tuning the substrate specificity of enzymes ${ }^{454}$, as well as creating scaffolds for enzyme immobilization ${ }^{105}$. General protein engineering strategies for biocatalysts in EFCs have been summarized in detail in two recent reviews ${ }^{196,455}$. More attention here will be paid on increasing the poor stability of EFCs. In detail, protein engineering can enable the stabilization of enzymes, by modifying the enzymes' structures in order to introduce more strong bonds, remove unfavorable steric effects, and remove potential degradation sites ${ }^{456}$. For example, a homodimeric pyrrolquinoline quinone GDH from Acinetobacter calcoaceticus was engineered by changing a single amino acid to cysteine, so that the lifetime of the EFC was greatly extended to $152 \mathrm{~h}$, more than 6-fold that of the EFC 
employing the wild-type. Enhanced disulfide bond formation of the active enzyme dimer may explain this result ${ }^{457}$. Additional work demonstrated that the thermal stability could be increased by introducing a hydrophobic interaction in the interface of the two subunits in this enzyme through two amino acid substitutions ${ }^{458}$. Direct evolution has been applied to Saccharomyces cerevisiae Lac by introducing mutations in the second coordination sphere of $\mathrm{T} 1$ to increase the resistance to chloride ions, making it more suitable to be used for EFCs working in physiological fluids ${ }^{459}$. It was also reported that protein oligomerisation is a potential means of increasing the stability of the bioelectrode ${ }^{460}$.

In addition to increasing the stability of engineered enzymes, other studies have focused on replacing expensive nicotinamide-based cofactors $\left(\mathrm{NAD}^{+}\right.$or $\left.\mathrm{NADP}^{+}\right)$required in MET-based EFCs with inexpensive and stable biomimics. In order to use these biomimetic cofactors, the cofactor preference of the respective oxidoreductase has to be altered. For example, Banta et al. engineered an alcohol dehydrogenase from Pyrococcus furiosus to utilize the biomimic cofactor nicotinamide mononucleotide $(\mathrm{NMN}){ }^{461}$. Compared to natural cofactors, such biomimic cofactors are smaller with faster rates of diffusion. In addition to increases in stability, gains in the performance of the NMN-mediated EFC were observed possibly due to improved rates of cofactor diffusion, despite a decreased turnover rate of the engineered enzyme. Zhang et al. changed the cofactor specificity of 6-phosphogluconate dehydrogenase from its natural cofactor NADP to NAD through a rational design strategy. The best mutant exhibited a $\sim 60,000$-fold reversal of the cofactor selectivity from NADP to NAD, and the associated EFC possessed an increase in power density and enhanced stability at high temperature ${ }^{462}$. Cofactor engineering not only can address the issue of unstable natural cofactors used in EFCs, but can also improve rates of mass transfer and the overall cost of EFCs. 


\subsection{Microbial surface displayed enzymes as biocatalysts to enhance EFCs' stability}

\subsubsection{Microbial surface display}

Microbial surface display refers to the biotechnology of introducing foreign peptides or proteins of interest on the surfaces of microbes by fusing them with appropriate anchoring protein motifs ${ }^{463,464}$, which is capable of maintaining their relatively independent spatial structure and biological activity. The microbial surface display system is usually composed of a passenger protein (target protein), an anchor protein (carrier protein) and host microbes (Figure 17). To date, varying anchor proteins such as ice nucleation protein (INP) ${ }^{465}$, Lpp-OmpA ${ }^{466}, \mathrm{EstA}^{467}$, and $\mathrm{OmpC}^{468}, \mathrm{OmpA}^{469}$ have been used. Microbial surface display is classified into phage display, yeast display and bacterial display, which enables foreign peptides or proteins to directly interact with substrate without passing through the outer membrane by means of genetic engineering. Moreover, this strategy can help to improve the stability of displayed proteins due to the immobilization on the surface of biomaterial support $^{470,471}$. So far, microbial surface display has been widely applied in live-vaccines ${ }^{472}$,

peptide or protein library screening ${ }^{473}$, bioadsorbents ${ }^{474}$, whole-cell biocatalysts ${ }^{475}$ and biosensors ${ }^{158,476}$. 
A)

Passenger

)

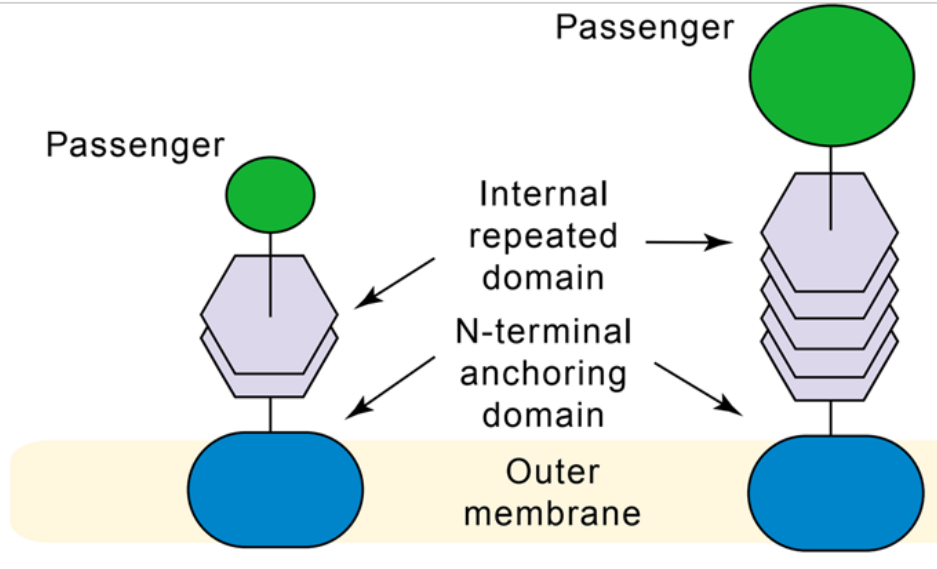

B)

Poly-histidine linker



Figure 17. A) Schematic representation of cell surface display system using INP, which is an example of the N-terminal fusion method. The INP is the most stable and useful carrier to express foreign proteins as large as $60 \mathrm{kDa}$. B) Cell-surface display system using E. coli outer membrane protein $\mathrm{C}$, which is a representative example of sandwich fusion method. In this system, poly-histidine (poly-His) peptides of up to 162 amino acids could be inserted into the seventh external loop (L7) of OmpC and could be efficiently exposed on the E. coli cell surface. Reprinted with permission. ${ }^{463}$ Copyright 2003 Elsevier.

\subsubsection{Efficient EFCs based on microbial surface displayed enzyme as biocatalysts}

As the entire microbe is used as the whole-cell biocatalyst, the enzymes expressed on the cell surface can exhibit improved stability when compared to that of free enzymes ${ }^{472-475}$. Thus, EFCs using whole-cell biocatalyst are expected to improve their performance, in particular in 
long-term operational stability. Additionally, without the need for enzyme purification, the enzyme-expressing whole-cell based biocatalysts have also been used for the construction of $\mathrm{EFCs}^{477-479}$. Alfonta's group displayed GOx on yeast cell surface using a-agglutinin as an anchor motif for EFC construction ${ }^{477}$. The randomly distributed GOx showed interesting advantages over unmodified yeast or purified GOx. Under the same condition, the engineered yeast based EFC showed an increased OCV of ca. $0.88 \mathrm{~V}$ in comparison to ca. $0.73 \mathrm{~V}$ for purified GOx. The improved performance was probably derived from the synergistic effect of both the GOx on the yeast surface and the imobilization of the metabolic output of the yeast cells for power production. It should be mentioned here that the microbe mainly serves as a stabilizing element ${ }^{479}$, different from MFCs which utilize an entire microorganism (also called electricigens) to convert the chemical energy of organic matter for electricity ${ }^{400-482}$. Recently, dehydrogenases have attracted significant attention as the reactions are not affected by the presence of oxygen. Liu et al. have described a number of reports on dehydrogenases, with xylose dehydrogenase $(\mathrm{XDH})^{483}, \mathrm{GDH}^{484,485}$, glutamate dehydrogenase ${ }^{486}$ and $\mathrm{FoDH}^{487}$ being successfully displayed on the surface of E. Coli. Biosensors ${ }^{483,486,488-491}$ and one-compartment biofuel cells ${ }^{286,492,493}$ have been prepared using these surface displayed dehydrogenases. Direct energy conversion from xylose was successfully achieved using XDH surface displayed E. coli (XDH-bacteria) based EFC, composed of XDH-bacteria immobilized on poly(brilliant cresyl blue)(PBCB)/MWCNTs modified glassy carbon electrode (GCE) (XDH-bacteria/PBCB/MWCNTs/GCE) based bioanode and a free BOD based biocathode ${ }^{492}$. Under optimized condition, a $\mathrm{P}_{\max }$ of $63 \mu \mathrm{W} \mathrm{cm}{ }^{-2}$ at $0.44 \mathrm{~V}$ was obtained, $60 \%$ higher than that of the free enzyme based $\mathrm{EFC}^{492}$ (Figure 18). It is noteworthy that this EFC could retain $85 \%$ of its maximal power after $12 \mathrm{~h}$ of continuous operation. A rationally designed XDH mutant NA-1 with improved thermostability was anchored on the bacterial surface $^{493}$. After $12 \mathrm{~h}$ operation, $88 \%$ of its maximal power was retained ${ }^{493}$. In another report, a bacterial surface displayed GDH mutant (mutant-GDH-bacteria) was immobilized onto MWCNTs as bioanode ${ }^{286}$. This EFC showed a $\mathrm{P}_{\max }$ of $55.8 \mu \mathrm{W} \mathrm{cm} \mathrm{cm}^{-2}$ at $0.45 \mathrm{~V}$ and an OCV of $0.80 \mathrm{~V}$ in $10 \mathrm{mM}$ glucose. The as-fabricated EFC retained $84 \%$ of $\mathrm{P}_{\max }$ even after continuous 
operation for $55 \mathrm{~h}$, benefitting from the high stability of the bacterial displayed GDH mutant $^{286}$.


Figure 18. A) Schematic illustration of a XDH-bacteria/PBCB/MWCNTs/GCE based bioanode. B) Dependence of the power density on the cell voltage of a free-XDH/ $\mathrm{PBCB} / \mathrm{MWCNTs} / \mathrm{GCE}$ bioanode based EFC in the absence (a) and the presence of $30 \mathrm{mM}$ xylose solution (b); free-XDH/PBCB/MWCNTs/GCE bioanode based EFC in the absence (c), and the presence of $30 \mathrm{mM}$ xylose solution (d); and XDH-bacteria/PBCB/MWCNTs/GCE bioanode based EFC in 0.1M PBS quiescent solution containing $30 \mathrm{mM}$ xylose and $10 \mathrm{mM}$ $\mathrm{NAD}^{+}$under $\mathrm{O}_{2}$-saturated condition (e). Reprinted with permission ${ }^{492}$. Copyright 2013 Elsevier.

Sequential enzymes refer to two or more enzymes involved in catalyzing cascade reactions sequentially and coordinately, for example, glucoamylase (GA)/GOx, ADH/FDH, and GOx/ horseradish peroxidase (HRP). Recently, EFCs based on sequential enzymes raised great interests. For instance, a membraneless starch $/ \mathrm{O}_{2} \mathrm{EFC}$ based on bioanode by co-immobilizing commercial GA and GOx ${ }^{494}$ as well as white rice/ $\mathrm{O}_{2} \mathrm{EFC}$ based on the multi-immobilization of GOx, alpha amylase and GA on a carbon paste electrode ${ }^{495}$, was developed. Nevertheless, it is complicated to co-immobilize two or three enzymes at the same time, as the spatial orientation of the enzymes cannot be controlled. Alfonta et al. displayed GA and GOx on yeast surface, respectively, to obtain GA-yeast and GOx-yeast, and then constructed a two-chamber $\mathrm{EFC}^{496}$. However, the $\mathrm{P}_{\max }$ was only about $3 \mu \mathrm{W} \mathrm{cm}{ }^{-2}$, probably due to the low catalytic efficiency arising from the spatial barrier between GA and GOx. The same group 
further co-expressed $\mathrm{ADH}$ and formaldehyde dehydrogenase (FormDH) on yeast cells using cohesin-dockerin interactions ${ }^{173}$. Subsequently, an EFC was fabricated using the displayed $\mathrm{ADH} /$ FormDH cascade based bioanodes and copper oxidase based biocathodes, however, a low power density $\left(<3 \mu \mathrm{W} \mathrm{cm}{ }^{-2}\right)$ was achieved ${ }^{173}$ because the surface patterning of the enzymes together with their orientation were not considered. It should be mentioned here that, the controlled co-displayed cascade enzymes should be superior to randomly displayed cascade enzymes as the enzyme cascades assembled on the cell would enable reactants to transfer between active sites of the enzymes efficiently, which makes great sense in biocatalysis and bioelectro-synthesis.

\subsection{Strategies for enzyme protection against $\mathrm{O}_{2}$ and reactive oxygen species (ROS)}

While oxygen is the oxidant mainly used as the cathodic substrate in EFCs where it is reduced to water, it can seriously affect the performance of the anode in EFCs if operating in a single compartment configuration. Reactive oxygen species (ROS, such as $\mathrm{O}_{2}{ }^{-}$), produced by the incomplete reduction of $\mathrm{O}_{2}$, can seriously affect the activity of enzymes. The majority of sugar/ $\mathrm{O}_{2}$ based EFCs rely on flavoprotein oxidases (e.g. GOx and lactate oxidase (LOx)) carrying a flavin cofactor tethered in the protein that utilizes $\mathrm{O}_{2}$ as an electron acceptor, producing $\mathrm{H}_{2} \mathrm{O}_{2}$ in the process ${ }^{497,498}$. The response of biosensors ${ }^{499}$ that rely on DET or MET generally does not detect $\mathrm{H}_{2} \mathrm{O}_{2}$ which can have a deleterious effect on the enzymes in the system ${ }^{500,501}$. Scodeller et al. found that exogenous peroxide reduced the electrocatalytic $\mathrm{O}_{2}$ reduction current at an Os-complex modified polymer mediated Trametes trogii Lac biocathode by ca. 20\% 502. $\mathrm{H}_{2} \mathrm{O}_{2}$ irreversibly inhibited the activity of a biocathode with immobilized Myrothecium $s p$. BOD, whereas a reversible deleterious effect was found with $T \nu \mathrm{Lac}{ }^{503}$. The underling mechanism of inhibition is still unclear.

In the case of implantable EFCs, the generation of $\mathrm{H}_{2} \mathrm{O}_{2}$ is also undesirable as it is toxic to the surrounding tissue ${ }^{3}$. Removal of $\mathrm{H}_{2} \mathrm{O}_{2}$ can be achieved via catalytic decomposition by catalase $31,34,41,383$ on the bioanode. It should be noted that there are EFCs based on $\mathrm{H}_{2} \mathrm{O}_{2}$-reducing 
biocathodes ${ }^{504-507}$. For example, GOx can catalyze the oxidation of glucose producing $\mathrm{H}_{2} \mathrm{O}_{2}$, which is electroenzymatically reduced into water by immobilized peroxidases ${ }^{333}$.

Re-engineering of oxygen-sensitive flavoprotein oxidases reduces the effect of oxygen ${ }^{508}$. The conversion of $\mathrm{O}_{2}$ into $\mathrm{H}_{2} \mathrm{O}_{2}$ involves two electrons and two protons transferred from the reduced flavin ${ }^{509}$. The active site binding pocket of AnGOx contains Glu412, His516, His559, and FAD ${ }^{510}$. His516 in the active site of native GOx is protonated and positively charged and is likely responsible for the electrostatic stabilization of the transition state for stepwise single-electron transfer between $\mathrm{FADH}^{-}$and $\mathrm{O}_{2}{ }^{511}$. The replacement of His516 by alanine by site-directed mutagenesis resulted in a 217-fold decrease in $\mathrm{k}_{\mathrm{cat}} / \mathrm{K}_{\mathrm{M}}\left(\mathrm{O}_{2}\right)$ at $\mathrm{pH} 5^{511}$. Gutierrez et al. identified four oxygen/mediator (quinone diimine) activity related positions in $A n \mathrm{GOx}$, which were close to the FAD domain and situated at the oxygen entry ${ }^{512}$. Simultaneous site saturation at the four positions by two rounds of directed evolution and ultra high-throughput screening resulted in a 37-fold decreased oxygen dependency, while retaining the catalytic efficiency for redox mediators and thermostability ${ }^{512}$. Sode et al. analyzed an oxygen-binding structural model of $P a \mathrm{GOx}$ and predicted that eight functional residues were involved in the oxidative half reaction ${ }^{513}$. Mutagenesis analysis by alanine substitution of these residues and subsequent activity assays indicated that the Ser114Ala mutant possessed the highest dehydrogenase performance with a $31 \%$ decrease in oxidase activity ${ }^{513}$. Bimutation at Ser114 and Phe334 in mutated PaGOx resulted in a 11-fold decrease in activity towards oxygen in comparison with the wild-type counterpart ${ }^{514}$. To simultaneously decrease the $\mathrm{O}_{2}$ sensitivity and maintain high activity towards glucose with artificial mediators, a double mutation was performed upon Val564, which is a nonpolar site to guide oxygen binding, and Lys $424{ }^{515}$, which allows enhancement of the electron transfer rate between $\mathrm{Os}$ redox polymer and $\mathrm{PaGOx}{ }^{516}$. The methodology to predict the oxygen access pathway to screen for mutants has been employed with other flavoprotein oxidases. For example, Aerococcus viridans lactate oxidase bearing a A96L mutant showed a significant decrease in oxidase activity using molecular oxygen as the electron acceptor, accompanied with a slight increase in activity using ferricyanide as the mediator ${ }^{517}$. 
Alternatively, oxygen-insensitive dehydrogenase modified bioanodes can avoid the undesirable issues arising by $\mathrm{H}_{2} \mathrm{O}_{2}{ }^{84}$. NAD-dependent $\mathrm{GDH}$ has been widely used for biosensors and EFCs based on the successful reduction of the overpotential for the regeneration of $\mathrm{NAD}^{+}$. However, the cofactor is not tightly bound to the enzyme limiting its application for implantable devices. The utilization of $\mathrm{NAD}^{+}$as a cofactor is also constrained by the complicated electrochemical regeneration of $\mathrm{NAD}^{+}$as the cofactor itself undergoes irreversible oxidation ${ }^{518}$. GDH using PQQ as the bound cofactor holds promise for use in an EFC ${ }^{221,499,519-522}$. DET of PQQ-GDH can be achieved by means of suitable enzyme immobilization ${ }^{221,520,523}$. FAD-dependent GDH (FAD-GDH) has been widely utilized in EFCs ${ }^{283,524-533}$. Milton et al. found that a GOx based membrane-less EFC initially had a higher power density than a FAD-GDH based EFC, while the FAD-GDH based EFC possessed better operating stability (after $24 \mathrm{~h}$ continuous operation) ${ }^{525}$. This confirms the negative effects of GOx bioanodes producing $\mathrm{H}_{2} \mathrm{O}_{2}$ on $\mathrm{BOD}^{525}$ and $\mathrm{Lac}^{530}$ biocathodes. $\mathrm{PDH}^{266,306,534-537}$ and $\mathrm{CDH}$ are other options for oxygen-inert bioanodes. $\mathrm{CDH}$ can catalyze several carbohydrates (glucose, lactose and cellobiose), and is promising as a versatile bioanode catalyst to simultaneously oxidize various fuels ${ }^{277}$. PDH shows a broad substrate specificity including glucose, xylose, galactose etc., and can catalyze the oxidation of sugar anomers at the C-2 and C-3 carbons of the sugar ${ }^{538}$.

Other enzymes are highly sensitive to $\mathrm{O}_{2}$ themselves, which is the case of most hydrogenases which are inactivated in the presence of $\mathrm{O}_{2}$, limiting the large-scale development of $\mathrm{H}_{2} / \mathrm{O}_{2}$ EFCs to replace Pt based catalysts suffering from scarcity and inhibition ${ }^{88}$. [NiFe] hydrogenases are the most efficient hydrogenases for $\mathrm{H}_{2}$ oxidation. Many studies have been made to produce $\mathrm{O}_{2}$-tolerant mutants, but none of these mutants are sufficiently resistant to be used as bioanodes $88,134,228$. One strategy is to purify oxygen tolerant hydrogenases, such as the membrane-bound [NiFe]-hydrogenases isolated from the bacteria Ralstonia eutropha, E. coli or Aquifex aeolicus ${ }^{65,67,338}$. The tolerance of these hydrogenases has been mainly ascribed to a [4Fe-3S] cluster in close proximity to the active site different from the cluster found in the sensitive hydrogenases, and able to provide the extra electrons required to reduce $\mathrm{O}_{2}$ into water as soon as it attacks the 
active site. However, even when using these $\mathrm{O}_{2}$-tolerant hydrogenases, inactivation by $\mathrm{O}_{2}$ occurs, although this is a reversible and fast process. A strategy to refill electrons to deactivated hydrogenase was proposed by Armstrong and coworkers, using an additional bioanode ${ }^{539}$. Nevertheless, a membrane separator was necessary to avoid cross diffusion of $\mathrm{O}_{2}$, and inactivation of the hydrogenases. Effectively, ROSs formed due to oxygen reduction at the carbon surface held at low potentials were found however to deactivate hydrogenase irreversibly ${ }^{335}$. Upper layers of 3D porous carbon matrix are believed to help to scavenge ROSs before they reach enzymes inside the pores ${ }^{67}$. It found that the hydrogenase encapsulated inside a 3 D porous matrix displays 4-6 times more stability against ROS than that on a $2 \mathrm{D}$ electrode.

To prevent the oxygen-induced damage on $\mathrm{O}_{2}$ sensitive hydrogenases, the employment of a "redox hydrogel shield" has been recently proposed by Schuhmann and Lubitz et al. (Figure 19) ${ }^{540}$. A specifically designed viologen-based redox polymer with a low potential catalyzes oxygen reduction at the polymer surface, thus preventing the inner enzyme modification layer from $\mathrm{O}_{2}$ damage and high-potential deactivation. Further, detailed characterization and numerical simulation were applied to reveal the underlying protection mechanism ${ }^{379}$. Protection has been successfully achieved for $[\mathrm{NiFe}]{ }^{540,541},[\mathrm{FeFe}]^{542}$ and $\left[\mathrm{NiFeSe}{ }^{444,543}\right.$ hydrogenases. However, the effect of byproducts such as superoxide and hydrogen peroxide that are derived from partial oxygen reduction should be taken into account ${ }^{542}$. Similar methodologies can be extended to develop a double layered lactose biosensor comprised of an inner $\mathrm{CDH}$ and outer GOx layer separately ${ }^{544}$. The outer GOx layer can remove a high concentration of glucose up to $140 \mathrm{mM}$, that is also the substrate of $\mathrm{CDH}$, enabling the system to operate as a reliable lactose sensor. 




Figure 19. Schematic drawing of the double protection of hydrogenases by a viologen based redox hydrogel shield. Active and inactive hydrogenases are indicted by open and filled circles, respectively. Assumed steady-state concentration curves of reduced viologen (blue solid line), $\mathrm{H}_{2}$ (green dash line) and $\mathrm{O}_{2}$ (red dash line) are shown. Reprinted with permission ${ }^{540}$. Copyright 2014 Nature Publishing Group.

\subsection{Anti-biofouling of implantable glucose $/ \mathrm{O}_{2}$ EFCs}

Implantable glucose/ $\mathrm{O}_{2}$ EFCs in blood suffer from biofouling process involving adsorption of layers of proteins and whole cells etc. that can impair the rate of diffusion of glucose and thus reduce the power output. Electrodes can be chemically modified with anti-biofouling layers that are hydrophilic (such as ethylene oxide functioning groups) or zwitterionics ${ }^{545,546}$. A range of coating membranes including $\mathrm{Nafion} \circledast$, cellulose acetate, chitosan, fibronectin and poly(styrene-sulphonate)/poly(l-lysine) have been evaluated for their ability to reduce levels of biofouling, using albumin in solution ${ }^{547}$. Fibronectin showed the best anti-biofouling effects with no significant differences in the voltammetric waves of $\left[\mathrm{Ru}\left(\mathrm{NH}_{3}\right)_{6}\right]^{3+}$ after exposing to albumin. The use of an anti-biofouling conductive polymer, poly(sulfobetaine-3,4-ethylenedioxythiophene) 
(PSBEDOT) which can be used to immobilize GOx is of interest ${ }^{548}$. PSBEDOT bears zwitterionic sulfobetaine side chains, resulting in a significant anti-biofouling electrode with only $8.4 \%$ protein adsorption in $100 \%$ human blood plasma compared to a control electrode without zwitterionic side chains (PEDOT). The electrochemical response to glucose in human blood plasma at a PSBEDOT-GOx based electrode was twice of a PEDOT-GOx electrode. It should be noted that modifications with anti-biofouling polymers may hinder the rate of ET and the diffusion of the substrate. Alternatively, nanoporous structured electrodes with similar pore sizes to the macromolecules (such as albumin) can repel proteins ${ }^{549}$, leaving the inner pores available for small molecules (such as redox mediators and fuels).

Blood clotting, occurring when placing foreign EFCs in the blood circulation, causes significant disturbance for the glucose transport. It requires the bioelectrodes to be biocompatible causing no inflammatory reactions when implanted in extra-cellular fluids between organs ${ }^{39}$. It's more challenging to make a hemocompatible surface to be implanted in the blood avoiding to destruct blood components ${ }^{39}$. Cosnier's group utilized dialysis bags to wrap carbon-based electrodes to prevent the leakage of immobilized species which were then placed in a Dacron ${ }^{\circledR}$ sleeve to improve biocompatibility ${ }^{31,34}$. However, the employment of dialysis bags requires a large volume EFC. The coating of biocompatible polymer layers, e.g. chitosan ${ }^{550}$ and collagen, etc. is another route ${ }^{8}$. Miyake et al. introduced a 2-methacryloyloxyethyl phosphorylcholine (MPC)-polymer coating to make carbon electrodes biocompatible ${ }^{341}$, without which obvious blood clotting was observed after $2 \mathrm{~h}$ immersion in blood. A needle-type glucose/ $\mathrm{O}_{2} \mathrm{EFC}$ in a rabbit vein displayed a power output of $0.42 \mu \mathrm{W}$ at $0.56 \mathrm{~V}$, while the cell without a MPC coating had ca. $40 \%$ lower in power ${ }^{341}$. The decreased performance was likely attributed to the presence of blood clots.

Cadet et al. tested Os-complex modified polymer mediated glucose/ $\mathrm{O}_{2}$ EFCs in 30 anonymized and disease-free whole blood samples ${ }^{273}$. A cellulose dialysis bag was placed on the EFCs. Comparison of the faradaic signal from the Os complexes in buffer and in blood showed that 
both possessed well-defined redox waves, with a $27 \mathrm{mV}$ larger peak separation in blood. This was explained by interferences caused by endogenous human blood constituents, a reversible process as the electrochemical waves were recovered by transferring the electrodes from blood to buffer. The lower catalytic response of the bioelectrodes in blood was mainly attributed to mass transport limitation as both currents increased with stirring rate. Ascorbate interference ${ }^{551}$ upon the biocathode was not observed ${ }^{273}$, which may be explained by the high selectivity of the bioelectrode with the Os-complex modified polymer. Over the course of $6 \mathrm{~h}$ continuous operation in blood ${ }^{273}$, the dialysis bag protected both enzymes, retaining twice the response of the unprotected system.

Non-invasive EFCs (Figure 20) operating in saliva ${ }^{552-554}$, sweat ${ }^{48}$ and tear ${ }^{142,506}$ are of interest as activators for wearable medical devices. Unlike implantable EFCs, non-invasive devices do not come into contact with blood and do not involve skin piercing, tissue damage or cause pain. Such biodevices are typically not exposed to the immune system so that tissue inflammatory responses can be avoided. They are also called "wearable EFCs" ${ }^{47}$, can be easily discarded and replaced and generally are flexible structures, with adequate oxygen supply. An interesting example is a contact lens supported microelectronic systems for glucose concentration monitoring in tears that was proposed in $2013{ }^{555}$. Xiao et al. reported a flexible lactate/ $\mathrm{O}_{2} \mathrm{EFC}$ on nanoporous gold electrodes that was mounted onto commercially available contact lenses and produced electricity for more than $5.5 \mathrm{~h}$ in a solution of artificial tears ${ }^{556}$. Other examples are tattoo ${ }^{48}$ and textile ${ }^{49}$ based EFCs producing electricity from human sweat based lactate. However, this approach is still not an effective solution for powering implantable medical devices. Another emerging group of skin borne EFCs are those using solid-state hydrogel electrolytes with preloaded sugars, which can generate biopower when the human subject is not perspiring ${ }^{50,557-560}$. 


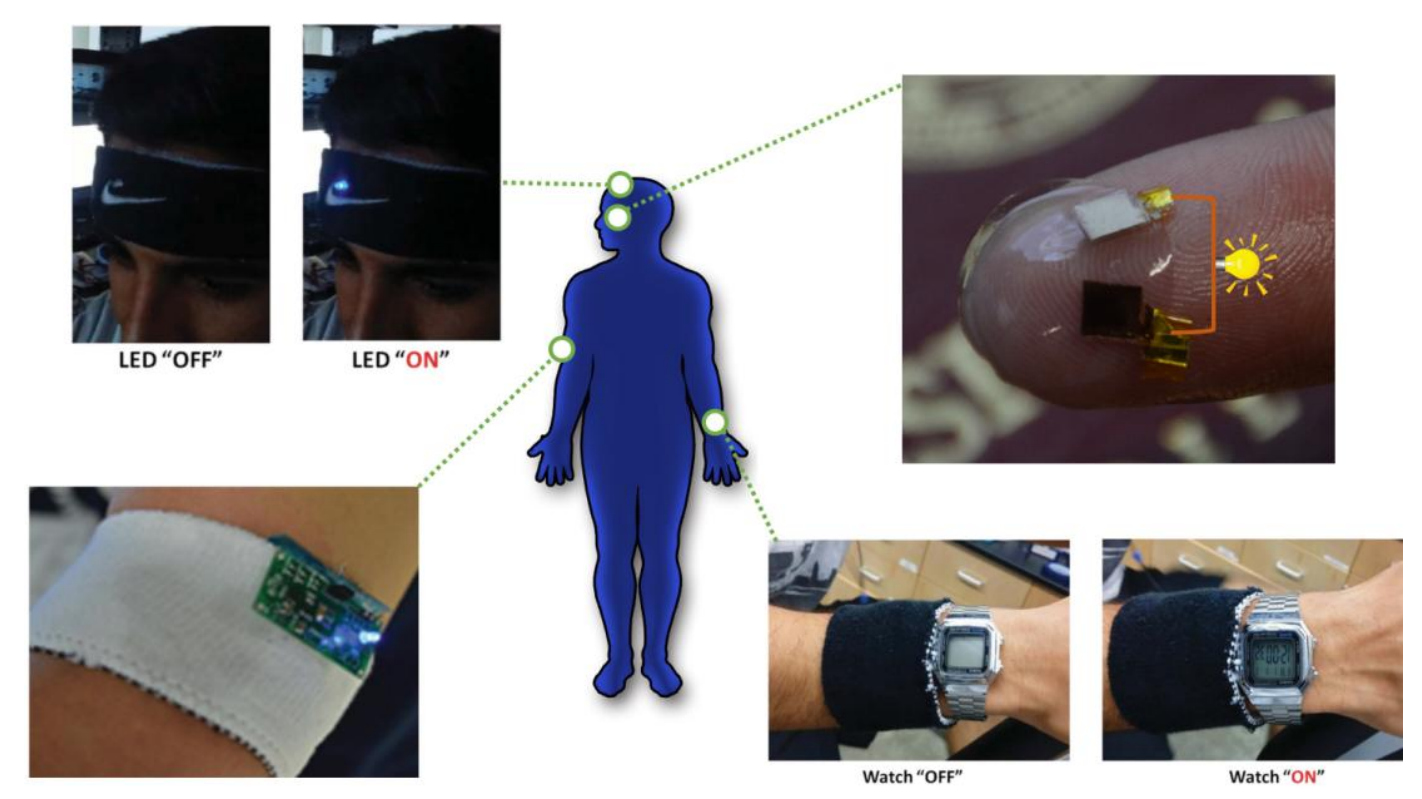

Figure 20. Various wearable lactate EFCs that are possible to be fueled with lactate in tears or sweats. Reprinted with permission ${ }^{49,54,556}$. Copyright 2014, 2017 Royal Society of Chemistry; Copyright 2018 American Chemical Society.

\section{Approaches for the improvement of EFCs' cell voltage}

An additional critical challenge of EFCs is that their output voltages are generally incompatible with the values required to operate commercially available microelectronic devices (1-3 $\left.\mathrm{V}^{143,561}\right)$, although transistors requiring an operating voltage of $0.5 \mathrm{~V}$ and even lower have been developed 562-564. The OCV of a biofuel cell is limited by the thermodynamic values for the species used as fuel and oxidant. In the standard state, the relationship between the standard Gibbs free energy change $\Delta \mathrm{G}^{0}\left(\mathrm{~kJ} \mathrm{~mol}^{-1}\right)$ and $\mathrm{E}^{\mathrm{o}}(\mathrm{V})$ can be expressed by the equation ${ }^{565}$ :

$$
\left|\Delta \mathrm{G}^{0}\right|=n \mathrm{FE}^{0}
$$

$\left|\Delta G^{0}\right|$ of biochemical reactants have been summarized by Alberty et al. ${ }^{566}$ For example, a glucose/ $\mathrm{O}_{2} \mathrm{EFC}$ using GOx or GDH as bioanode catalysts undergoes an overall reaction:

$$
\beta-\mathrm{D}-\text { Glucose }\left(\mathrm{C}_{6} \mathrm{H}_{12} \mathrm{O}_{6}\right)+\frac{1}{2} \mathrm{O}_{2} \rightarrow \mathrm{D}-\text { Gluconolactone }\left(\mathrm{C}_{6} \mathrm{H}_{10} \mathrm{O}_{6}\right)+\mathrm{H}_{2} \mathrm{O}
$$

As $\left|\Delta \mathrm{G}^{0}\right|$ for eq. 13 is $227.23 \mathrm{~kJ} \mathrm{~mol}^{-1}$ at $25^{\circ} \mathrm{C}, \mathrm{pH} 7$ and $0.1 \mathrm{M}$ glucose, $\mathrm{n}=2$, the value of $\mathrm{E}_{\text {cell }}^{\mathrm{o}}$ is $1.18 \mathrm{~V}$. 
The typical polarization curve of an EFC (Figure 21B) presents a wide range of information such as the experimental OCV and maximum cell current/current density. In practice, the registered $\mathrm{OCV}$ of an EFC is much lower than $\mathrm{E}^{\mathrm{o}}$ due to the presence of three types of potential losses, namely kinetic $\left(\eta_{\text {act }}\right)$, ohmic losses $\left(I \sum R\right)$ and mass transport losses $\left(\eta_{\text {diff }}\right)$, in the system. The relationship between registered $\mathrm{OCV}$ and $\mathrm{E}^{\mathrm{o}}$ can be determined by ${ }^{11}$ :

$$
\mathrm{OCV}=\mathrm{E}^{o}-\eta_{\text {act }}-\mathrm{I} \sum \mathrm{R}-\eta_{\text {diff }}
$$

where $\eta_{\text {act }}$ is the overpotential required to overcome energy barriers on the electrode-electrolyte interfaces; $\eta_{\text {act }}=\eta_{\text {act,a }}+\eta_{\text {act, }, \text {, }}$, where the subscripts a and $\mathrm{c}$ indicate the anodic and cathodic reactions, respectively; $\sum \mathrm{R}$ is the sum of all resistances associated with current $\mathrm{I}$ that flows through the electrodes, electrolyte and various interconnections; $\eta_{\text {diff }}$ is the mass transport based overpotential due to reactant diffusion limitations. Three characteristic regions, distinguished by the different governing overpotentials $\left(\eta_{\text {act }}, I \sum R\right.$ and $\left.\eta_{\text {diff }}\right)$, can be found in a typical polarization curve (Figure 21B $)^{7}$.

In region a) governed by $\eta_{\text {act }}$ where the reactants (fuels and oxidants) are abundant and the current is low, the rate of reaction is solely controlled by the rate of heterogenous ET. The current $I$ can be expressed using the Butler-Volmer equation ${ }^{567}$ :

$$
I=A i_{0}\left(\exp \left(-\frac{\alpha n F \eta_{a c t, c}}{R T}\right)-\exp \left(\frac{(1-\alpha) n F \eta_{a c t, a}}{R T}\right)\right)
$$

Where $i_{0}$ is the exchange current density.

In the high overpotential region $(>118 / \mathrm{n} \mathrm{mV})$, the Butler-Volmer equation can be simplified to the Tafel equation ${ }^{567}$ :

$$
\eta_{a c t}=b \log _{10}\left(\frac{i}{i_{0}}\right)
$$

where $i$ is the current density; $b$ is the Tafel slope $\left(\mathrm{mV} \mathrm{dec}^{-1}\right)$. Eq. 16 allows the determination of $i_{0}$ and $b^{568}$. Further, the rate of electron transfer rate $\left(k_{\mathrm{et}}\right)$ can be obtained from:

$$
i_{0}=n F A k_{e t}[S]
$$


Visually, the measured OCV can be read from the power density profile or the polarization curve (Figure 21), which is consistent with the difference between the onset potential for the oxidation of the fuel and the reduction of the oxidant, respectively ${ }^{7}$. Although the term "onset potential" is quite fuzzy due to the difficulties in defining the exact starting points for electrochemical oxidation or reduction ${ }^{11}$, it can be obtained, in practice, by comparing the potential-current profiles of bioelectrodes in the presence and absence of the substrate (Figure 21A $)^{11,569}$. Thus, the measured OCV can also be expressed as ${ }^{80}$ :

$$
\begin{aligned}
& \mathrm{OCV}=\mathrm{E}_{c}^{\text {onset }}-\mathrm{E}_{a}^{\text {onset }}-\mathrm{I} \sum \mathrm{R}_{e} \\
& \mathrm{OCV}=\left(\mathrm{E}_{c}-\eta_{c}\right)-\left(\mathrm{E}_{a}-\eta_{a}\right)-\mathrm{I} \sum \mathrm{R}_{e}
\end{aligned}
$$

where $\mathrm{R}_{\mathrm{e}}$ is the resistance, $\mathrm{E}_{\mathrm{c}}{ }^{\text {onset }}$ and $\mathrm{E}_{\mathrm{a}}{ }^{\text {onset }}$ are the observed onset potentials for the cathode and anode, $\mathrm{E}_{\mathrm{c}}$ and $\mathrm{E}_{\mathrm{a}}$ are the thermodynamic onset potentials at the cathode and anode, respectively, $\eta_{\mathrm{b}}$ and $\eta_{\mathrm{a}}$ are the overpotentials for cathode and anode, respectively. Eqs. 18 and 19 suggest the strategies to maximize OCV of a single EFC via bring the starting potentials of both bioanode and biocathode closer to those of the enzymes/cofactors ${ }^{4}$.

For biocathodes, MCOs based bioelectrodes undergoing DET with low overpotentials are widely adopted. Fungal Lac possesses a much higher redox potential (up to $0.78 \mathrm{~V}$ vs. SHE ${ }^{29}$ ) for the $\mathrm{T} 1 \mathrm{Cu}$ site than that of $\mathrm{BOD}$ (ca. $0.67 \mathrm{~V}$ vs. SHE ${ }^{21}$ ). BOD exhibits higher activity at physiological conditions (i.e. neutral $\mathrm{pH}$ ) and is less sensitive to chloride ions at neutral $\mathrm{pH}$, making it a better candidate for implantable EFCs. Lac is usually inhibited by chloride ions and is active in the $\mathrm{pH}$ range 4-5, making it a suitable choice for non-implantable applications. On the bioanode side, NAD-dependent dehydrogenase can present a low onset potential due to the low formal potential of $\mathrm{NAD}^{+}\left(\mathrm{E}^{\mathrm{o}^{\prime}}\right.$ NADH/NAD+: $-0.33 \mathrm{~V}$ vs. SHE $\left.{ }^{570}\right)$. FAD-dependent dehydrogenases ( $\mathrm{E}_{\text {FADH2/FAD }}^{\mathrm{o}}:-0.18 \mathrm{~V}^{571}$ ) are preferred over PQQ ( $\mathrm{E}_{\text {PQQH2/PQQ: }}^{\mathrm{o}^{\prime}} 0.12 \mathrm{~V}$ vs. SHE $\left.{ }^{572}\right)$ due to the lower redox potential. While $\mathrm{O}_{2}$-sensitive $[\mathrm{NiFe}]$ hydrogenases present a very low overpotential for $\mathrm{H}_{2}$ oxidation, the $\mathrm{O}_{2}$-tolerant membrane ones oxidize $\mathrm{H}_{2}$ at potentials around $150 \mathrm{mV}$ higher. 
Nevertheless, $\mathrm{H}_{2} / \mathrm{O}_{2}$ EFCs based on $\mathrm{O}_{2}$-tolerant hydrogenases and BOD possess OCV greater than $1.1 \mathrm{~V}^{88}$.



Electrocatalytic reduction of oxidant at the cathode

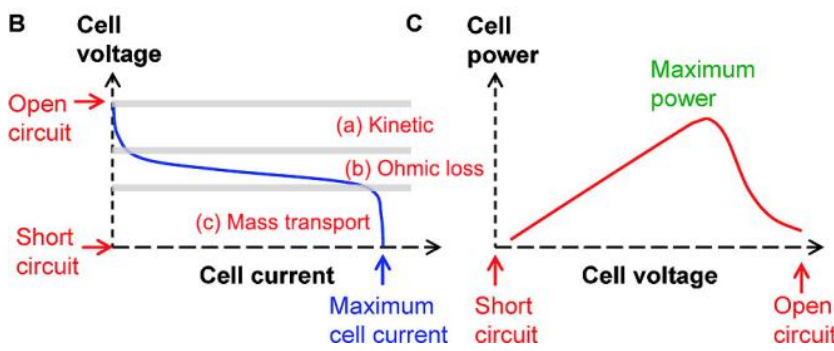

Figure 21. (A) Polarisation curves of a bioanode and biocathode. (B) Voltage-current profile (B) and power density-voltage profile of an EFC. Key parameters of an EFC are highlighted. Reprinted with permission ${ }^{7}$. Copyright 2018 American Chemical Society.

\subsection{Mediator optimization}

EFCs based on DET bioelectrocatalysis on both the anode and cathode without the involvement of mediators are promising as they avoid any possible toxicity effects of the mediator, in particular in the use of implantable EFCs ${ }^{125,573}$. They generally display a higher OCV than those based on MET. However, the following examples show that MET can generate higher OCVs. In an example of a FDH modified electrode, the presence of ubiquinone as the mediator with a redox potential in between those of FAD (-0.034 V vs. SHE at pH 5.5) and heme $(0.135 \mathrm{~V}$ vs. SHE at $\mathrm{pH}$ 5.5) enables transfer of electrons directly from FAD directly rather than via the heme 218. In other words, due to the lower energy barrier to be overcome, the external low-redox-potential mediators substitute the role of the "built-in mediator" (heme) in communicating with FAD catalytic center, leading to lower overpotentials. Similarly, when using an Os redox polymer with a lower potential than that of heme ${ }^{277}, M t \mathrm{CDH}$ modified electrode had 
a $150 \mathrm{mV}$ lower onset potential for MET than that of DET. These examples emphasize the importance of engineering the redox potential of mediators for enzymes undergoing MET.

ET between the enzyme and the mediator is driven by the mediator-induced overpotential $\left(\Delta \mathrm{E}_{\mathrm{et}}\right)$, i.e. the difference between the redox potential of the enzyme catalytic active center and the mediator ${ }^{574}$. According to Marcus theory, the rate constant $\left(\mathrm{k}_{\mathrm{et}}\right)$ between an enzyme and mediator is given by ${ }^{325}$

$$
\mathrm{k}_{e t}=\mathrm{Z} \exp \left[-\frac{\left(\lambda-n \mathrm{~F} \Delta \mathrm{E}_{e t}\right)^{2}}{4 \lambda R T}\right]
$$

where $\mathrm{Z}$ is the frequency factor, $\lambda$ is the molecular reorganization free energy, $\mathrm{R}$ is the gas constant, $\mathrm{T}$ is the absolute temperature. Mathematically, the relationship between $\mathrm{k}_{\mathrm{et}}$ and $\Delta \mathrm{E}_{\mathrm{et}}$ displays a quadratic behaviour, with a region where $\mathrm{k}_{\mathrm{et}}$ increases with $\Delta \mathrm{E}_{\mathrm{et}}$ (normal region) and an inverted region where $\mathrm{k}_{\mathrm{et}}$ decreases with increasing $\Delta \mathrm{E}_{\mathrm{et}}$. Typically, the inverted region is not observed, which is likely due to the fact that at high $\Delta \mathrm{E}_{\text {et }}$ the biocatalytic reaction becomes mass-transport limited ${ }^{574} . \Delta \mathrm{E}_{\mathrm{et}}$ should be as high as possible to enhance the current density, but that can result in higher overpotentials, lowering the OCV. Improvements in the OCV and power density of a mediated EFC are mutually exclusive ${ }^{64}$, thus, the value of $\Delta \mathrm{E}_{\mathrm{et}}$ should be optimised to yield both a high current density and a high OCV.

In practice, an efficient combination of redox mediator and enzyme requires optimization experiments ${ }^{325,575}$. The co-immobilization of redox mediator and enzyme is essential for implantable EFCs using MET based bioelectrodes to avoid leakage. Redox polymers introduced by Heller et al. ${ }^{271,576,577}$ are the most important group of mediators for the construction of EFCs 578. Redox polymers also act as the host matrix to immobilize enzymes via electrostatic interaction, entrapment and/or chemically cross-linking, resulting in a catalytic film permeable to the fuels and necessary ions ${ }^{271}$. Polymer backbones bearing organometallic groups (e.g. Os complex ${ }^{578}$, ferrocene ${ }^{64,389,579,580}$, cobaltocene ${ }^{581}$ ), organic dyes (e.g. viologen ${ }^{540,581}$, phenothiazine ${ }^{507,582}$ ) and quinone ${ }^{64,583,584}$ have been synthesized for mediated bioelectrodes. The 
utilization of redox polymers allows electrical connection of multilayered enzymes, irrespective of enzyme orientation, leading to higher current output. The formal potentials of redox polymers (Figure 22) are determined primarily by the type and the nature of the covalently bound redox couples, ${ }^{120,271,585}$ and redox polymers based bioelectrodes with optimized redox potentials can be fabricated by using the appropriately designed redox species. ${ }^{50,586-588}$.

Bartlett and Pratt developed a comprehensive model of the diffusion and kinetic effects within a uniform layer containing both immobilized GOx and mediator on an electrode surface ${ }^{589}$, which can be used to understand the limiting factors in redox-polymer based bioelectrodes. Experimental variables including enzyme loading, film thickness, substrate concentration, mediator concentration and electrode potential can be considered using this approach. The summary case diagrams can be used to predict the electrochemical response of an electrode under specified experimental conditions. It thus important, although difficult, to accurately determine the effective enzyme and mediator concentrations on the electrode.



Figure 22. The range of redox potentials of enzyme cofactors and common mediators. Reprinted with permission from ref ${ }^{120}$. Copyright 2018 Elsevier.

An example is Os-complex based redox polymers, whose formal potential can be adjusted by using different ligands. ${ }^{11,586,590,591}$ Schuhmann's group has reported a series of Os-complex modified polymers with redox potentials, for example, close to $0.2 \mathrm{~V}$ vs. SHE. ${ }^{587,592}$ Consequently, glucose $/ \mathrm{O}_{2}$ EFCs with OCVs of $0.50 \sim 0.54 \mathrm{~V}$ were developed. In addition, EFCs with improved OCVs of $0.6 \sim 0.8 \mathrm{~V}$ could be achieved by combination of 
phenothiazine- or quinone derivative-modified redox polymer based bioanodes. ${ }^{64}$ For more negative redox potentials, viologen based redox polymers can be used, for example with a redox potential of -0.3 vs. SHE (Figure 23). ${ }^{540} \mathrm{H}_{2} / \mathrm{O}_{2}$ EFCs with high OCVs of $\sim 1 \mathrm{~V}$ were fabricated by combination of such viologen-based polymer MET-type $\mathrm{H}_{2}$ bioanodes and DET-type $\mathrm{O}_{2}$ biocathodes. Various ligands were synthesized to tune the redox potentials of the hydrogels ${ }^{593}$. A similar viologen polymer-modified bioelectrode has been reported by Kano's group for formate oxidation by $\mathrm{FDH}$, and a formate/ $\mathrm{O}_{2} \mathrm{EFC}$ with an OCV of $1.2 \mathrm{~V}$ was recorded. ${ }^{15}$ Compared to the DET-type bioelectrocatalysis and MET-type bioelectrocatalysis using free mediators, redox polymer-based bioelectrocatalysis possess advantages such as rapid rates of ET, low levels of mediators and/or enzyme leakage. From this viewpoint, selection or development of redox polymers with specific properties, for example, low redox potential, high biocompatibility and stability, good permeablility for mass transfer of substrate and product through the film (i.e. tunable polymer film thickness), as well as high affinity to enzymes, have significant potential.
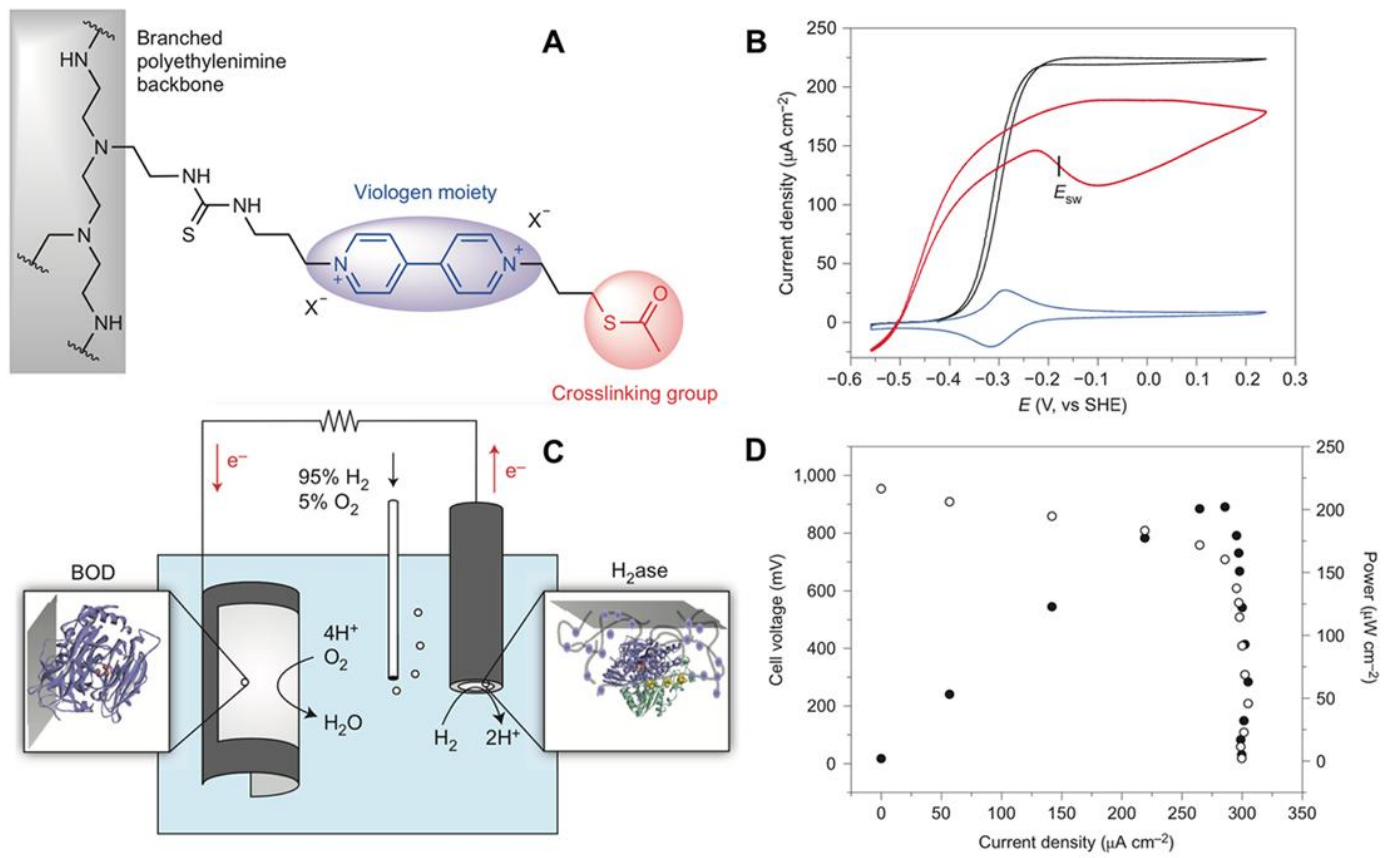

Figure 23. Viologen-redox polymer-based $\mathrm{H}_{2} / \mathrm{O}_{2}$ biofuel cell. A) Chemical structure of viologen-modified polymer; B) Cyclic voltammograms of a [NiFe] hydrogenase from Desulfovibrio vulgaris Miyazaki F/polymer electrode under $\mathrm{H}_{2}$ (black) and $\mathrm{CO}$ (blue) and a covalently modified electrode with the hydrogenase in DET configuration (red). Experimental 
conditions: electrode rotation rate of 2,000 rpm, $\mathrm{pH} 7.0,40{ }^{\circ} \mathrm{C}, 1 \mathrm{mV} \mathrm{s}^{-1}$ and 1 bar of $\mathrm{H}_{2}$ (black and red traces), $20 \mathrm{mV} \mathrm{s}^{-1}$ and 1 bar of $\mathrm{CO}$ (blue trace). C) Schematic diagram of a single compartment EFC, $\mathrm{H}_{2} / \mathrm{O}_{2}$ mixed feed, hydrogenase-coated anode and oversized $\mathrm{O}_{2}$-reducing BOD-coated cathode. D) Cell voltage (open circles) and power density (filled circles) versus current density for the $\mathrm{H}_{2} / \mathrm{O}_{2}$ EFCs. Reproduced with permission from ref ${ }^{540}$. Copyright 2014 Nature Publishing Group.

Gallaway et al. combined experimental data with numerical modeling to examine the influence of $\Delta \mathrm{E}_{\mathrm{et}}$ of a series of Os redox polymers on $T \nu \mathrm{Lac}$ catalyzed oxygen reduction at $\mathrm{pH} 4{ }^{574}$. When $\Delta \mathrm{E}_{\text {et }}$ was lower than $300 \mathrm{mV}$, a larger $\Delta \mathrm{E}_{\mathrm{et}}$ significantly enhanced the power output. The optimum $\Delta \mathrm{E}_{\mathrm{et}}$ to obtain maximum power from an $\mathrm{EFC}$ using a non-limiting anode with an onset potential of $0 \mathrm{~V}$ vs. SHE was $0.17 \mathrm{~V}$. Zafar et al. studied the effects of using five different Os-complex modified polymers with redox potentials over the range -0.07 to $+0.36 \mathrm{~V}$ vs. SHE on the performance of a mediated $A m \mathrm{PDH}$ bioanode ${ }^{535} . \Delta \mathrm{E}_{\mathrm{et}}$ and the structural properties including flexibility and length of the tether were crucial for the overall performance. The results indicated that an Os-complex modified polymer with a moderately high $\Delta \mathrm{E}_{\mathrm{et}}$, companying with a long tether between the Os complex and the backbone with a greatly enhanced ET collision frequency, gave higher current densities. An Os-complex modified polymer with a redox potential of $0.14 \mathrm{~V}$ vs. SHE, that is slightly (ca. $20 \mathrm{mV}$ ) higher than that of the bound FAD of AmPDH (-0.17 V), was selected to be optimal mediator in terms of high current density and low onset potential ${ }^{535}$. On comparing six different Os-complex modified polymers with redox potentials ranging from -0.02 to $0.49 \mathrm{~V}$ vs. SHE for Glomerella cingulata FAD-GDH ${ }^{590}$, two Os-complex modified polymers with redox potentials of 0.31 and $0.42 \mathrm{~V}$ vs. SHE yielded the highest current densities. The above reports imply that a moderate $\Delta \mathrm{E}_{\mathrm{et}}$ is responsible for the high current density, allowing for optimization of OCV further. Heller suggested a $\Delta \mathrm{E}_{\mathrm{et}}$ of $50 \mathrm{mV}$ for implantable glucose $/ \mathrm{O}_{2}$ EFCs using both mediated bioanodes and biocathode in order to obtained practical OCVs ${ }^{578}$. Based on such a design, Heller's group reported a glucose $/ \mathrm{O}_{2} \mathrm{EFC}$ presenting an OCV of ca. $1 \mathrm{~V}$ and a $\mathrm{P}_{\max }$ of $350 \mu \mathrm{W} \mathrm{cm}{ }^{-2}$ in an air-saturated $15 \mathrm{mM}$ glucose solution at $\mathrm{pH} 5$ ${ }^{578}$. It consisted of a GOx bioanode mediated by an Os-complex modified polymer with a 
13-atom-long flexible tether (ca. 0 V vs. SHE) ${ }^{594}$, and a Lac biocathode with an Os-complex modified polymer bearing 8-atom-long tethers (ca. $0.75 \mathrm{~V}$ vs. SHE). It should be noted that $\mathrm{O}_{2}$ can to be reduced at the low-redox-potential Os-complex modified polymer producing $\mathrm{H}_{2} \mathrm{O}_{2}{ }^{595}$ and thus the interference of $\mathrm{O}_{2}$ on the mediator itself ${ }^{540}$ should also be considered.

Minteer et al. compared soluble 1,2- and 1,4- naphthoquinone (NQ) mediated FAD-GDH bioanodes and found that 1,2-NQ derivatives had larger catalytic current densities, which can be explained by the high values of $\Delta \mathrm{E}_{\mathrm{et}}{ }^{64}$. The obtained current densities between different NQ species with different structural reorganization or enzymatic affinity effects were not comparable. On grafting 1,2- and 1,4-NQ-epoxy groups onto linear LPEI, the NQ-2-LPEI showed a lower mediated bioelectrocatalytic response in comparison to that of NQ-4-LPEI. The NQ-4-LPEI/GDH-FDH bioanode displayed an onset potential of ca. -0.01 V vs. SHE. In combination with a non-limiting carbon felt/BOD biocathode, the resultant EFC registered an OCV of ca. $0.87 \mathrm{~V}$ and a $\mathrm{P}_{\max }$ of $2.3 \pm 0.2 \mathrm{~mW} \mathrm{~cm}{ }^{-2}$ in air-saturated $100 \mathrm{mM}$ glucose at $\mathrm{pH} 6.5^{64}$.

\subsection{Serial connection}

Unlike microbial fuel cells which often encounter voltage reversal when stacked, EFCs do not have this issue ${ }^{596}$. Serial assembly of conventional fuel cells can be employed with EFCs to amplify the output voltage, while the connection in parallel can enable increases in current density ${ }^{182,597}$. Sakai reported a carbon fibre based glucose/ $\mathrm{O}_{2} \mathrm{EFC}$ with $\mathrm{NAD}^{+}$-dependent GDH, BOD and mediators co-immobilized showing a $\mathrm{P}_{\max }$ of $1.45 \pm 0.24 \mathrm{~mW} \mathrm{~cm}^{-2}$ at $0.3 \mathrm{~V}$ and a OCV of $0.8 \mathrm{~V}$ in the presence of $400 \mathrm{mM}$ glucose ${ }^{52}$. A stacked cell of two individual EFCs allowed the successful operation of a radio-controlled car (16.5 g) and a memory-type Walkman continuously for more than $2 \mathrm{~h}$. A microfluidic biobattery utilizing NAD ${ }^{+}$-dependent $\mathrm{ADH}$ and $\mathrm{Pt} / \mathrm{C}$ at the bioanode and cathode, respectively, generated an OCV of $0.93 \mathrm{~V}$ which was increased to $1.44 \mathrm{~V}$ on connecting two cells in series ${ }^{598} . \mathrm{A} \mathrm{H}_{2} / \mathrm{O}_{2} \mathrm{EFC}$ composed of two stacks of four cells in parallel with OCV and $\mathrm{P}_{\max }$ of $2.09 \mathrm{~V}$ and $7.84 \mathrm{~mW}$, respectively, was used to power an electronic clock and red LEDs for $8 \mathrm{~h}$ with no decrease in light intensity ${ }^{182}$. Miyake et al. reported 
a laminated stack of EFCS consisting of fructose oxidizing bioanode fabrics, air-breathing biocathode fabrics and a sandwiched hydrogel layer containing fructose ${ }^{50}$. A triple-layer stack produced an OCV of $2.09 \mathrm{~V}$, a 2.8 -fold increase over that of a single set cell $(0.74 \mathrm{~V})$ and a $\mathrm{P}_{\max }$ of $0.64 \mathrm{~mW}$ at $1.21 \mathrm{~V}$, that was able to power LEDs. Paper based EFCs are cost-effective as disposable devices ${ }^{19}$. A screen-printed circular-type EFC system, composed of a series of 5 individual cells with a single cell OCV of $0.57 \mathrm{~V}$, generated an OCV of $2.65 \mathrm{~V}$ and illuminated an LED directly ${ }^{599}$.

The overall performance of interconnected EFC in serial is limited by the weakest EFC. Preparation of the stack needs to be carefully controlled and reproducible, especially with regard to material preparation and to the immobilization of the enzymes. Moreover, the serial-connection of EFCs with metal leads requires that individual EFC be isolated properly to avoid short-circuits introduced by ion-conductive electrolytes. MacVittie et al. prepared a buckypaper supported EFC composed of a PQQ-GDH bioanode and a Lac biocathode achieving an OCV of $0.54 \mathrm{~V}^{33}$. Two EFCs implanted in a serial-configuration in separate claws of a lobster showed an OCV of only ca. $0.5-0.6 \mathrm{~V}$. The potential of the serially connected EFCs was limited due to the ionic conductivity in the same body. Serial connection of two lobsters bearing EFCs resulted in a voltage of ca. $1 \mathrm{~V}$. A fluidic system comprised of five EFCs connected in series was able to generate an OCV of ca. $3 \mathrm{~V}$ sufficient to activate a pacemaker. Similarly, an implantable glucose $/ \mathrm{O}_{2} \mathrm{EFC}$ in a clam registered an OCV of ca. $300-400 \mathrm{mV}$ and the serial connection of 3 "electrified" clams afforded an OCV of ca. $800 \mathrm{mV}^{36}$. Due to the above-mentioned constraint caused by the ionic conductivity, serial configuration has been primarily used for in vitro experimentation. As a solution, superhydrophobic surface may help to build ionic isolation between signal cells ${ }^{597}$. Three glucose/ $\mathrm{O}_{2}$ EFCs (OCV: $0.35 \mathrm{~V}$ ) were series-connected on a fluidic chip and air valves were introduced between cells by a lotus leaf-like superhydrophobic structure. The possible output voltage was ca. $1 \mathrm{~V}$. 


\subsection{Employment of external boost converter}

The output voltage of an EFC can be boosted by externally connecting a charge pump as a DC-DC converter ${ }^{600}$. For example, a voltage-doubler operates by charging of two capacitors in parallel separately followed by discharge in series. Many examples in the recent litterature illustrate this concept. In 2013, Southcott et al. prepared a fluidic glucose $/ \mathrm{O}_{2} \mathrm{EFC}$ with an $\mathrm{OCV}$ of $0.47 \mathrm{~V}$ in a serum solution that mimick the human blood circulatory system ${ }^{601}$. A single EFC was connected to a combination of a charge pump with a DC-DC converter, which increased the voltage from 0.3 to $2 \mathrm{~V}$ and from 2 to $3 \mathrm{~V}$, respectively. The resultant device enabled the continuous operation of a commercial pacemaker ${ }^{601}$. Coupling of a glucose $/ \mathrm{O}_{2}$ biobattery with a charge pump and a capacitor resulted in $1.8 \mathrm{~V}$ electric pulses at different intervals determined by the fuel concentration ${ }^{381}$. A commercial BQ25504 boost converter could amplify an input voltage in the range of $0.3-0.5 \mathrm{~V}$ up to $3 \mathrm{~V}^{34}$. The EFC/boost converter/capacitor assembly enabled a glucose $/ \mathrm{O}_{2}$ EFC implanted in rats with an OCV of $0.57 \mathrm{~V}$ to intermittently power a digital thermometer (power consumption: $50 \mu \mathrm{A}$ at $1.5 \mathrm{~V}$ ) and a LED (4.1 mA at $2.9 \mathrm{~V})$. The output of other reported glucose/ $\mathrm{O}_{2}$ EFCs could be amplified using similar boosting systems (OCV from $0.6 \mathrm{~V}$ to $2.3 \mathrm{~V}$ ) to power a wireless transmitter ${ }^{323}$, from $0.3 \mathrm{~V}$ to $1.8 \mathrm{~V}$ to power a $\mathrm{LED}^{324}$ and from $0.145 \mathrm{~V}$ to $2.586 \mathrm{~V}$ for a glucometer ${ }^{561}$. Those amplified voltage output can be used directly to activate microelectronic devices.

Lactate/ $\mathrm{O}_{2}$ EFCs consuming sweat and tear lactate are of interest to activate wearable medical devices. A power unit composed of an EFC/voltage booster couple can be easily combined into wearable devices. For example, two lactate $/ \mathrm{O}_{2}$ biobatteries with an $\mathrm{OCV}$ of $0.67 \mathrm{~V}$ in parallel were able to generate $6 \mu \mathrm{W}$ at $0.376 \mathrm{~V}$, which was scaled up to $3.2 \mathrm{~V}$ to periodically to illuminate a blue LED bubble requiring $2.5 \mathrm{~V}$ and $0.5 \mathrm{~mA}^{49}$. A lactate/ $\mathrm{O}_{2} \mathrm{EFC}$ with an $\mathrm{OCV}$ of $0.87 \mathrm{~V}$ was used to provide the operational voltage of an electronic watch (ca. $3 \mathrm{~V}){ }^{506}$. A biobattery using real sweat lactate with an OCV of $0.5 \mathrm{~V}$ was coupled with a DC-DC converter/capacitor circuit to produce a $3.5 \mathrm{~V}$ pulse with a width of $53 \mathrm{~s}^{54}$. 
EFCs based on other fuels have also been reported. A lactose $/ \mathrm{O}_{2} \mathrm{EFC}$ with an OCV of $0.73 \mathrm{~V}$ has been integrated with a voltage amplifier and a capacitor ${ }^{289}$, which was coupled into wireless carbohydrate and oxygen biosensor platforms with a threshold of $44 \mu \mathrm{A}$ and $0.57 \mathrm{~V}$. Three fructose $/ \mathrm{O}_{2}$ EFCs with an $\mathrm{OCV}$ of $0.7 \mathrm{~V}$ in series generated $2 \mathrm{~mW}$ and $2 \mathrm{~V}$, which was integrated with a minipotentiostat containing a DC-DC converter with an output voltage of $4 \mathrm{~V}^{388}$. The integrated device enabled an oxygen sensor allowing ten measurements in the pulse mode without any disturbances. $\mathrm{A} \mathrm{H}_{2} / \mathrm{O}_{2} \mathrm{EFC}$ registering an $\mathrm{OCV}$ of $1.12 \mathrm{~V}$ can be boosted over $6 \mathrm{~V}$ to power a wireless device sending data every $25 \mathrm{~s}$ in a course of 7 hours continuous operation ${ }^{382}$.

It can be concluded that most reports utilized the DC-DC converter/capacitor junction with a pulse function. Only few reports have claimed that they can power an external device continuously ${ }^{601}$. It should be noted that part of the generated power is consumed by the DC-DC converter as a price of the voltage boost, posing extra demand on EFC's output power ${ }^{561}$. The commercial BQ25504 boost converter requires a net current input from 10 to $100 \mu \mathrm{A}{ }^{34}$, requiring a high-current-density EFC. Otherwise a larger size electrode is required, hindering the miniaturization of the implantable power source. The need for an external circuit increases the size of the devices, making device encapsulation more complex.

\section{Conclusions and perspectives}

EFCs are expected to be one of the next-generation energy conversion systems because they utilize bioavailable, renewable and diverse biocatalysts and biosourced fuels, operate under mild and safe conditions, and possess high theoretical energy-conversion efficiencies. In this review, we discuss four main obstacles, namely low energy density, power density, stability and output voltage, that hinder the successful development of EFCs and summarize a range of potential solutions. In spite of their high activity, the high specificity of enzymes typically restricts the ability of an enzyme to catalyze just a single reaction, leading to low fuel utilization efficiency and thus low power densities in single-enzyme based EFCs. A rationally designed bioanode 
consisting of enzyme cascades or multi-step pathways has been proposed to improve the overall energy density. Additionally, approaches that utilise engineered enzymes to increase their catalytic performance, "wiring" enzyme with favourable orientation to facilitate improved rates of direct electron transfer, utilising nanomaterials to achieve high enzyme loadings, smart design of electrodes and cell for enhanced mass transfer, as well as constructing EFC and biocapacitor hybrid devices, have all been developed for high power density. A range of approaches ranging from enzyme immobilization to biochemical engineering have been investigated to extend the lifetime of EFCs. Microbial surface displayed enzymes, which are anchored on a cell surface mimicking the micro-environment that enzymes function in nature, are expected to provide enzymes with long term operational stability. Improved cell voltages have been realized by well-designed bioelectrode (MET or DET) with low overpotentials, series connection of cells, or external voltage boosters.

It should be noted that these obstacles are identified from the point of view of the measurable performance of EFCs'. Many of the strategies mentioned above can simultaneously address more than one practical issues. For example, enzyme cascades can also be used to improve the power density of an EFC while achieving the complete oxidation of the fuel ${ }^{124}$. Enzyme immobilization also plays a key role in increasing the power density of various DET-type EFCs as it is important to appropriately orient DET-capable enzymes to minimise the distances of electron transfer between enzymes and electrodes ${ }^{21}$. These combined strategies can generate synergistic effects to enhance the performance of EFCs and should be addressed in combination rather than individually.

In addition to increasing performance metrics of EFCs, expanding their functionalities is highly promising to enhance the practicability. As already mentioned, self-powered biosensors employing EFCs to function simultaneously as a power source and as a sensor offer the possibility to fabricate instrument-free (at least potentiostat-free) diagnostic systems ${ }^{86,151,602}$. A self-powered biosensor is generally based on the preparation of an EFC generating power that is 
proportional to the concentration of the analyte, which can be the fuel $\mathrm{l}^{324,443,507,603,604}$, inhibitor $^{605-607}$, activator, ${ }^{608}$ biorecognition element ${ }^{609-611}$ for enzymes used in the EFC. This type of biosensor is promising due to features of portability, miniaturization and low-cost. Operational stability issue can be overcome by fabricating disposable devices.

Rather than employing EFCs to power existing devices that require high power and voltage, new concepts, i.e. self-power bioelectronics ${ }^{149}$, which utilize EFCs directly to achieve specific functions can be more feasible for practical applications. Unlike batteries requiring careful encapsulation to avoid the direct contact of the battery active materials and the body, EFCs possess the merit of ease-of-miniaturization as the bioelectrodes can be used directly in the body. A recently reported EFC/supercapacitor system can function as a pulse generator to mimic a cardiac pacemaker delivering $10 \mu \mathrm{A}$ pulses for $0.5 \mathrm{~ms}$ at a frequency of $0.2 \mathrm{~Hz}^{283}$. This is different from previous attempts to use EFCs to power a commercial pacemaker ${ }^{33}$, which required a minimum voltage input of $3 \mathrm{~V}$. EFC based controlled drug release is an emerging area of interest. In preliminary studies, an iontophoretic system using buit-in EFCs allowed transdermal release of compounds into the skin ${ }^{558}$ and to heal skin wounds ${ }^{557}$. It should be possible to use implantable EFCs to generate electric stimuli to trigger in vivo release of drugs ${ }^{612}$. Recent work ${ }^{613-615}$ by Katz et al. using bioelectrodes for insulin release is of interest.

Enzymatic electrosynthesis ${ }^{616}$ in an EFC, or self-powered bioelectrosynthesis, enables simultaneous electrosynthesis of valuable chemicals and energy harvest. Rather than using an external high-power output, self-powered bioelectrosynthesis can enable the production of valuable chemicals circumventing external electricity input ${ }^{617}$. Minteer et al. reported the bioelectrocatalytic reduction of $\mathrm{N}_{2}$ to $\mathrm{NH}_{3}$ as the biocathode of a $\mathrm{H}_{2}$-fuelled $\mathrm{EFC}^{153}$. This spontaneous process to produce ammonia is of interest to explore alternatives to the Haber-Bosch process. A $\mathrm{H}_{2} /$ heptanal $\mathrm{EFC}$ reported recently revealed the ability to produce alkanes from aldehydes and alcohols ${ }^{152}$, opening the prospect of using EFCs to prepare renewable biofuels. Zhu et al. developed a self-powered system by combining an EFC and an enzymatic 
electrosynthesis cell and demonstrated the high-efficient production of 1-3,4-dihydroxyphenylalanine powered by glucose oxidation, suggesting that EFCs can be a promising power source for the synthesis of valuable chemicals and pharmaceuticals ${ }^{618}$.

Although there are significant obstacles to the development of EFCs, great opportunities to overcome these issues for practical applications are under investigation. Given that multidisplanar efforts have been taken to this prosperous topic, the time to transfer the lab-scale EFCs to real-life devices is not expected to be far away.

\section{Acknowledgements}

This work was financially supported by National Natural Science Foundation of China (Nos. 81673172, 21475144, 21275152 and 91227116 to A.L., Nos. 21706273, 21878324 to Z.Z.), Major Program of Shandong Province Natural Science Foundation (ZR2018ZC0125) to A.L., the CAS Pioneer Hundred Talent Program (Type C, reference \# 2016-081) to Z.Z., Platform Chimie NanoBio ICMG FR 2607 (PCN-ICMG) to S.C., and ANR (ENZYMOR-ANR-16-CE05- 0024) to E.L.. X.X. acknowledges a Government of Ireland Postgraduate Scholarship (GOIPG/2014/659) and a H. C. Ørsted COFUND fellowship.

\section{References}

(1) Carrette, L.; Friedrich, K. A.; Stimming, U. Fuel Cells-Fundamentals and Applications. Fuel Cells 2001, 1, 5-39.

(2) Windmiller, J. R.; Wang, J. Wearable Electrochemical Sensors and Biosensors: A Review. Electroanalysis 2013, 25, 29-46.

(3) Katz, E.; MacVittie, K. Implanted Biofuel Cells Operating in vivo - Methods, Applications and Perspectives - Feature Article. Energy Environ. Sci. 2013, 6, 2791-2803.

(4) Rasmussen, M.; Abdellaoui, S.; Minteer, S. D. Enzymatic Biofuel Cells: 30 Years of Critical Advancements. Biosens. Bioelectron. 2015, 76, 91-102.

(5) Calabrese Barton, S.; Gallaway, J.; Atanassov, P. Enzymatic Biofuel Cells for Implantable and Microscale Devices. Chem. Rev. 2004, 104, 4867-4886.

(6) Kim, J.; Jia, H.; Wang, P. Challenges in Biocatalysis for Enzyme-Based Biofuel Cells. Biotechnol. Adv. 2006, 24, 296-308.

(7) Cracknell, J. A.; Vincent, K. A.; Armstrong, F. A. Enzymes as Working or Inspirational 
Electrocatalysts for Fuel Cells and Electrolysis. Chem. Rev. 2008, 108, 2439-2461.

(8) Cosnier, S.; J. Gross, A.; Le Goff, A.; Holzinger, M. Recent Advances on Enzymatic Glucose/Oxygen and Hydrogen/Oxygen Biofuel Cells: Achievements and Limitations. J. Power Sources 2016, 325, 252-263.

(9) Cosnier, S.; Gross, A. J.; Giroud, F.; Holzinger, M. Beyond the Hype Surrounding Biofuel Cells: What's the Future of Enzymatic Fuel Cells? Curr. Opin. Electrochem. 2018, 148-155.

(10) Yahiro, A. T.; Lee, S. M.; Kimble, D. O. Bioelectrochemistry: I. Enzyme Utilizing Bio-Fuel Cell Studies. Biochim. Biophys. Acta 1964, 88, 375-383.

(11) Suzuki, A.; Mano, N.; Tsujimura, S. Lowering the Potential of Electroenzymatic Glucose Oxidation on Redox Hydrogel-Modified Porous Carbon Electrode. Electrochim. Acta 2017, 232, 581-585.

(12) Agnès, C.; Reuillard, B.; Le Goff, A.; Holzinger, M.; Cosnier, S. A Double-Walled Carbon Nanotube-Based Glucose $/ \mathrm{H}_{2} \mathrm{O}_{2}$ Biofuel Cell Operating under Physiological Conditions. Electrochem. Commun. 2013, 34, 105-108.

(13) Kamitaka, Y.; Tsujimura, S.; Setoyama, N.; Kajino, T.; Kano, K. Fructose/Dioxygen Biofuel Cell Based on Direct Electron Transfer-Type Bioelectrocatalysis. Phys. Chem. Chem. Phys. 2007, 9, 1793-1801.

(14) Ramanavicius, A.; Kausaite, A.; Ramanaviciene, A. Enzymatic Biofuel Cell Based on Anode and Cathode Powered by Ethanol. Biosens. Bioelectron. 2008, 24, 767-772.

(15) Sakai, K.; Kitazumi, Y.; Shirai, O.; Takagi, K.; Kano, K. High-Power Formate/Dioxygen Biofuel Cell Based on Mediated Electron Transfer Type Bioelectrocatalysis. ACS Catal. 2017, 7, 5668-5673.

(16) Xia, H.-q.; So, K.; Kitazumi, Y.; Shirai, O.; Nishikawa, K.; Higuchi, Y.; Kano, K. Dual Gas-Diffusion Membrane- and Mediatorless Dihydrogen/Air-Breathing Biofuel Cell Operating at Room Temperature. J. Power Sources 2016, 335, 105-112.

(17) Kontani, A.; Masuda, M.; Matsumura, H.; Nakamura, N.; Yohda, M.; Ohno, H. A Bioanode Using Thermostable Alcohol Dehydrogenase for an Ethanol Biofuel Cell Operating at High Temperatures. Electroanalysis 2014, 26, 682-686.

(18) Wang, X.; Roger, M.; Clement, R.; Lecomte, S.; Biaso, F.; Abriata, L. A.; Mansuelle, P.; Mazurenko, I.; Giudici-Orticoni, M. T.; Lojou, E.et al. Electron Transfer in an Acidophilic Bacterium: Interaction between a Diheme Cytochrome and a Cupredoxin. Chem. Sci. 2018, 4879-4891.

(19) Zhang, L.; Zhou, M.; Wen, D.; Bai, L.; Lou, B.; Dong, S. Small-Size Biofuel Cell on Paper. Biosens. Bioelectron. 2012, 35, 155-159.

(20) Ghindilis, A. L.; Atanasov, P.; Wilkins, E. Enzyme-Catalyzed Direct Electron Transfer: Fundamentals and Analytical Applications. Electroanalysis 1997, 9, 661-674.

(21) Falk, M.; Blum, Z.; Shleev, S. Direct Electron Transfer Based Enzymatic Fuel Cells. Electrochim. Acta 2012, 82, 191-202.

(22) Cass, A. E. G.; Davis, G.; Francis, G. D.; Hill, H. A. O.; Aston, W. J.; Higgins, I. J.; Plotkin, E. V.; Scott, L. D. L.; Turner, A. P. F. Ferrocene-Mediated Enzyme Electrode for Amperometric Determination of Glucose. Anal. Chem. 1984, 56, 667-671.

(23) Kano, K.; Ikeda, T. Fundamentals and Practices of Mediated Bioelectrocatalysis. Anal. Sci. 2000, 16, 1013-1021.

(24) Vincent, K. A.; Parkin, A.; Armstrong, F. A. Investigating and Exploiting the Electrocatalytic 
Properties of Hydrogenase. Chem. Rev. 2007, 107, 4366-4413.

(25) Volbeda, A.; Charon, M.-H.; Piras, C.; Hatachikian, E. C.; Frey, M.; Fontecilla-Camps, J. C. Crystal Sturcture of Nickel-Iron Hydrogenase from Desulfovbrio gigas. Nature 1995, 373, 580-587.

(26) Hibino, Y.; Kawai, S.; Kitazumi, Y.; Shirai, O.; Kano, K. Mutation of Heme c Axial Ligands in $D$-Fructose Dehydrogenase for Investigation of Electron Transfer Pathways and Reduction of Overpotential in Direct Electron Transfer-Type Bioelectrocatalysis. Electrochem. Commun. 2016, 67, 43-46.

(27) Kawai, S.; Yakushi, T.; Matsushita, K.; Kitazumi, Y.; Shirai, O.; Kano, K. The Electron Transfer Pathway in Direct Electrochemical Communication of Fructose Dehydrogenase with Electrodes. Electrochem. Commun. 2014, 38, 28-31.

(28) Kamitaka, Y.; Tsujimura, S.; Kataoka, K.; Sakurai, T.; Ikeda, T.; Kano, K. Effects of Axial Ligand Mutation of the Type I Copper Site in Bilirubin Oxidase on Direct Electron Transfer-Type Bioelectrocatalytic Reduction of Dioxygen. J. Electroanal. Chem. 2007, 601, 119-124.

(29) Shleev, S.; Tkac, J.; Christenson, A.; Ruzgas, T.; Yaropolov, A. I.; Whittaker, J. W.; Gorton, L. Direct Electron Transfer between Copper-Containing Proteins and Electrodes. Biosens. Bioelectron. 2005, 20, 2517-2554.

(30) Zebda, A.; Alcaraz, J.-P.; Vadgama, P.; Shleev, S.; Minteer, S. D.; Boucher, F.; Cinquin, P.; Martin, D. K. Challenges for Successful Implantation of Biofuel Cells. Bioelectrochem. 2018, 124, 57-72.

(31) Cinquin, P.; Gondran, C.; Giroud, F.; Mazabrard, S.; Pellissier, A.; Boucher, F.; Alcaraz, J.-P.; Gorgy, K.; Lenouvel, F.; Mathé, S.et al. A Glucose Biofuel Cell Implanted in Rats. PLOS ONE 2010, 5 , e10476.

(32) El Ichi, S.; Zebda, A.; Alcaraz, J. P.; Laaroussi, A.; Boucher, F.; Boutonnat, J.; Reverdy-Bruas, N.; Chaussy, D.; Belgacem, M. N.; Cinquin, P.et al. Bioelectrodes Modified with Chitosan for Long-Term Energy Supply from the Body. Energy Environ. Sci. 2015, 8, 1017-1026.

(33) MacVittie, K.; Halamek, J.; Halamkova, L.; Southcott, M.; Jemison, W. D.; Lobel, R.; Katz, E. From "Cyborg" Lobsters to a Pacemaker Powered by Implantable Biofuel Cells. Energy Environ. Sci. 2013, 6, 81-86.

(34) Zebda, A.; Cosnier, S.; Alcaraz, J.-P.; Holzinger, M.; Le Goff, A.; Gondran, C.; Boucher, F.; Giroud, F.; Gorgy, K.; Lamraoui, H. Single Glucose Biofuel Cells Implanted in Rats Power Electronic Devices. Sci. Rep. 2013, 3, 1516.

(35) Shoji, K.; Akiyama, Y.; Suzuki, M.; Nakamura, N.; Ohno, H.; Morishima, K. Biofuel Cell Backpacked Insect and Its Application to Wireless Sensing. Biosens. Bioelectron. 2016, 78, 390-395.

(36) Szczupak, A.; Halamek, J.; Halamkova, L.; Bocharova, V.; Alfonta, L.; Katz, E. Living Battery-Biofuel Cells Operating in vivo in Clams. Energy Environ. Sci. 2012, 5, 8891-8895.

(37) Rasmussen, M.; Ritzmann, R. E.; Lee, I.; Pollack, A. J.; Scherson, D. An Implantable Biofuel Cell for a Live Insect. J. Am. Chem. Soc. 2012, 134, 1458-1460.

(38) Castorena-Gonzalez, J. A.; Foote, C.; MacVittie, K.; Halámek, J.; Halámková, L.; Martinez-Lemus, L. A.; Katz, E. Biofuel Cell Operating in vivo in Rat. Electroanalysis 2013, 25, 1579-1584.

(39) Cosnier, S.; Le Goff, A.; Holzinger, M. Towards Glucose Biofuel Cells Implanted in Human Body for Powering Artificial Organs: Review. Electrochem. Commun. 2014, 38, 19-23.

(40) Babadi, A. A.; Bagheri, S.; Hamid, S. B. Progress on Implantable Biofuel Cell: Nano-Carbon 
Functionalization for Enzyme Immobilization Enhancement. Biosens. Bioelectron. 2016, 79, 850-860.

(41) Reuillard, B.; Le Goff, A.; Agnes, C.; Holzinger, M.; Zebda, A.; Gondran, C.; Elouarzaki, K.; Cosnier, S. High Power Enzymatic Biofuel Cell Based on Naphthoquinone-Mediated Oxidation of Glucose by Glucose Oxidase in a Carbon Nanotube 3D Matrix. Phys. Chem. Chem. Phys. 2013, 15, 4892-4896.

(42) Zhu, Z.; Kin Tam, T.; Sun, F.; You, C.; Percival Zhang, Y. H. A High-Energy-Density Sugar Biobattery Based on a Synthetic Enzymatic Pathway. Nat. Commun. 2014, 5, 3026.

(43) Kang, Z.; Jiao, K.; Cheng, J.; Peng, R.; Jiao, S.; Hu, Z. A Novel Three-Dimensional Carbonized PANI1600@CNTs Network for Enhanced Enzymatic Biofuel Cell. Biosens. Bioelectron. 2018, 101, 60-65.

(44) Gamella, M.; Koushanpour, A.; Katz, E. Biofuel Cells-Activation of Micro- and Macro-Electronic Devices. Bioelectrochem. 2018, 119, 33-42.

(45) Bandodkar, A. J.; Wang, J. Wearable Biofuel Cells: A Review. Electroanalysis 2016, 28, 1188-1200.

(46) Yu, Y.; Zhai, J.; Xia, Y.; Dong, S. Single Wearable Sensing Energy Device Based on Photoelectric Biofuel Cells for Simultaneous Analysis of Perspiration and Illuminance. Nanoscale 2017, 9, 11846-11850.

(47) Bandodkar, A. J. Review_-Wearable Biofuel Cells: Past, Present and Future. J. Electrochem. Soc. 2017, 164, H3007-H3014.

(48) Jia, W.; Valdés-Ramírez, G.; Bandodkar, A. J.; Windmiller, J. R.; Wang, J. Epidermal Biofuel Cells: Energy Harvesting from Human Perspiration. Angew. Chem. Int. Ed. 2013, 52, 7233-7236.

(49) Jia, W.; Wang, X.; Imani, S.; Bandodkar, A. J.; Ramirez, J.; Mercier, P. P.; Wang, J. Wearable Textile Biofuel Cells for Powering Electronics. J. Mater. Chem. A 2014, 2, 18184-18189.

(50) Miyake, T.; Haneda, K.; Yoshino, S.; Nishizawa, M. Flexible, Layered Biofuel Cells. Biosens. Bioelectron. 2013, 40, 45-49.

(51) Desmaële, D.; Renaud, L.; Tingry, S. A Wireless Sensor Powered by a Flexible Stack of Membraneless Enzymatic Biofuel Cells. Sens. Actuat. B: Chem. 2015, 220, 583-589.

(52) Sakai, H.; Nakagawa, T.; Tokita, Y.; Hatazawa, T.; Ikeda, T.; Tsujimura, S.; Kano, K. A High-Power Glucose/Oxygen Biofuel Cell Operating under Quiescent Conditions. Energy Environ. Sci. 2009, 2, 133-138.

(53) Majdecka, D.; Draminska, S.; Janusek, D.; Krysinski, P.; Bilewicz, R. A Self-Powered Biosensing Device with an Integrated Hybrid Biofuel Cell for Intermittent Monitoring of Analytes. Biosens. Bioelectron. 2018, 102, 383-388.

(54) Bandodkar, A. J.; You, J.-M.; Kim, N.-H.; Gu, Y.; Kumar, R.; Mohan, A. M. V.; Kurniawan, J.; Imani, S.; Nakagawa, T.; Parish, B.et al. Soft, Stretchable, High Power Density Electronic Skin-Based Biofuel Cells for Scavenging Energy from Human Sweat. Energy Environ. Sci. 2017, 10, 1581-1589.

(55)Zhu, Z.; Tam, T.; Zhang, Y. H. P. In Fundamentals and Application of New Bioproduction Systems; Zeng, A.-P., Ed.; Springer Berlin Heidelberg, 2013; Vol. 137.

(56) Sokic-Lazic, D.; Arechederra, R. L.; Treu, B. L.; Minteer, S. D. Oxidation of Biofuels: Fuel Diversity and Effectiveness of Fuel Oxidation through Multiple Enzyme Cascades. Electroanalysis 2010, 22, 757-764.

(57) Pinyou, P.; Conzuelo, F.; Sliozberg, K.; Vivekananthan, J.; Contin, A.; Pöller, S.; Plumeré, N.; 
Schuhmann, W. Coupling of an Enzymatic Biofuel Cell to an Electrochemical Cell for Self-Powered Glucose Sensing with Optical Readout. Bioelectrochem. 2015, 106, $22-27$.

(58) Merle, G.; Brunel, L.; Tingry, S.; Cretin, M.; Rolland, M.; Servat, K.; Jolivalt, C.; Innocent, C.; Seta, P. Electrode Biomaterials Based on Immobilized Laccase. Application for Enzymatic Reduction of Dioxygen. Mater. Sci. Eng. C 2008, 28, 932-938.

(59) Zebda, A.; Gondran, C.; Le Goff, A.; Holzinger, M.; Cinquin, P.; Cosnier, S. Mediatorless High-Power Glucose Biofuel Cells Based on Compressed Carbon Nanotube-Enzyme Electrodes. Nat. Commun. 2011, 2, 370.

(60) Shleev, S. Quo Vadis, Implanted Fuel Cell? ChemPlusChem 2017, 82, 522-539.

(61) Tokita, Y.; Nakagawa, T.; Sakai, H.; Sugiyama, T.; Matsumoto, R.; Hatazawa, T. Sony's Biofuel Cell. ECS Trans. 2008, 13, 89-97.

(62) Kwon, C. H.; Lee, S.-H.; Choi, Y.-B.; Lee, J. A.; Kim, S. H.; Kim, H.-H.; Spinks, G. M.; Wallace, G. G.; Lima, M. D.; Kozlov, M. E.et al. High-Power Biofuel Cell Textiles from Woven Biscrolled Carbon Nanotube Yarns. Nat. Commun. 2014, 5, 3928.

(63) Fujita, S.; Yamanoi, S.; Murata, K.; Mita, H.; Samukawa, T.; Nakagawa, T.; Sakai, H.; Tokita, Y. A Repeatedly Refuelable Mediated Biofuel Cell Based on a Hierarchical Porous Carbon Electrode. Sci. Rep. 2014, 4, 4937.

(64) Aquino Neto, S.; Hickey, D. P.; Milton, R. D.; De Andrade, A. R.; Minteer, S. D. High Current Density PQQ-Dependent Alcohol and Aldehyde Dehydrogenase Bioanodes. Biosens. Bioelectron. 2015, $72,247-254$.

(65) de Poulpiquet, A.; Ciaccafava, A.; Gadiou, R.; Gounel, S.; Giudici-Orticoni, M. T.; Mano, N.; Lojou, E. Design of a $\mathrm{H}_{2} / \mathrm{O}_{2}$ Biofuel Cell Based on Thermostable Enzymes. Electrochem. Commun. 2014, 42, 72-74.

(66) Xu, L.; Armstrong, F. A. Optimizing the Power of Enzyme-Based Membrane-Less Hydrogen Fuel Cells for Hydrogen-Rich $\mathrm{H}_{2}$-Air Mixtures. Energy Environ. Sci. 2013, 6, 2166-2171.

(67) Mazurenko, I.; Monsalve, K.; Infossi, P.; Giudici-Orticoni, M.-T.; Topin, F.; Mano, N.; Lojou, E. Impact of Substrate Diffusion and Enzyme Distribution in 3D-Porous Electrodes: A Combined Electrochemical and Modelling Study of a Thermostable $\mathrm{H}_{2} / \mathrm{O}_{2}$ Enzymatic Fuel Cell. Energy Environ. Sci. 2017, 10, 1966-1982.

(68) So, K.; Kitazumi, Y.; Shirai, O.; Nishikawa, K.; Higuchi, Y.; Kano, K. Direct Electron Transfer-Type Dual Gas Diffusion $\mathrm{H}_{2} / \mathrm{O}_{2}$ Biofuel Cells. J. Mater. Chem. A 2016, 4, 8742-8749.

(69) Szczesny, J.; Marković, N.; Conzuelo, F.; Zacarias, S.; Pereira, I. A. C.; Lubitz, W.; Plumeré, N.; Schuhmann, W.; Ruff, A. A Gas Breathing Hydrogen/Air Biofuel Cell Comprising a Redox Polymer/Hydrogenase-Based Bioanode. Nat. Commun. 2018, 9, 4715.

(70) So, K.; Kawai, S.; Hamano, Y.; Kitazumi, Y.; Shirai, O.; Hibi, M.; Ogawa, J.; Kano, K. Improvement of a Direct Electron Transfer-Type Fructose/Dioxygen Biofuel Cell with a Substrate-Modified Biocathode. Phys. Chem. Chem. Phys. 2014, 16, 4823-4829.

(71) Miyake, T.; Yoshino, S.; Yamada, T.; Hata, K.; Nishizawa, M. Self-Regulating Enzyme-Nanotube Ensemble Films and Their Application as Flexible Electrodes for Biofuel Cells. J. Am. Chem. Soc. 2011, $133,5129-5134$.

(72) Kizling, M.; Stolarczyk, K.; Tammela, P.; Wang, Z.; Nyholm, L.; Golimowski, J.; Bilewicz, R. Bioelectrodes Based on Pseudocapacitive Cellulose/Polypyrrole Composite Improve Performance of 
Biofuel cell. Bioelectrochem. 2016, 112, 184-190.

(73) Gai, P.; Ji, Y.; Chen, Y.; Zhu, C.; Zhang, J.; Zhu, J.-J. A Nitrogen-Doped Graphene/Gold Nanoparticle/Formate Dehydrogenase Bioanode for High Power Output Membrane-Less Formic Acid/O $\mathrm{O}_{2}$ Biofuel Cells. Analyst 2015, 140, 1822-1826.

(74) Handa, Y.; Yamagiwa, K.; Ikeda, Y.; Yanagisawa, Y.; Watanabe, S.; Yabuuchi, N.; Komaba, S. Fabrication of Carbon-Felt-Based Multi-Enzyme Immobilized Anodes to Oxidize Sucrose for Biofuel Cells. ChemPhysChem 2014, 15, 2145-2151.

(75) Deng, L.; Shang, L.; Wen, D.; Zhai, J.; Dong, S. A Membraneless Biofuel Cell Powered by Ethanol and Alcoholic Beverage. Biosens. Bioelectron. 2010, 26, 70-73.

(76) Selloum, D.; Tingry, S.; Techer, V.; Renaud, L.; Innocent, C.; Zouaoui, A. Optimized Electrode Arrangement and Activation of Bioelectrodes Activity by Carbon Nanoparticles for Efficient Ethanol Microfluidic Biofuel Cells. J. Power Sources 2014, 269, 834-840.

(77) Masa, J.; Schuhmann, W. Electrocatalysis and Bioelectrocatalysis - Distinction without a Difference. Nano Energy 2016, 29, 466-475.

(78) Saboe, P. O.; Conte, E.; Farell, M.; Bazan, G. C.; Kumar, M. Biomimetic and Bioinspired Approaches for Wiring Enzymes to Electrode Interfaces. Energy Environ. Sci. 2016, 10, 14-41.

(79) Moehlenbrock, M. J.; Minteer, S. D. Extended Lifetime Biofuel Cells. Chem. Soc. Rev. 2008, 37, 1188-1196.

(80) Luz, R. A.; Pereira, A. R.; de Souza, J. C.; Sales, F. C.; Crespilho, F. N. Enzyme Biofuel Cells: Thermodynamics, Kinetics and Challenges in Applicability. ChemElectroChem 2014, 1, 1751-1777.

(81) Pereira, A. R.; de Souza, J. C. P.; Iost, R. M.; Sales, F. C. P. F.; Crespilho, F. N. Application of Carbon Fibers to Flexible Enzyme Electrodes. J. Electroanal. Chem. 2016, 780, 396-406.

(82) Cooney, M. J.; Svoboda, V.; Lau, C.; Martin, G.; Minteer, S. D. Enzyme Catalysed Biofuel Cells. Energy Environ. Sci. 2008, 1, 320-337.

(83) Ha, S.; Wee, Y.; Kim, J. Nanobiocatalysis for Enzymatic Biofuel Cells. Top. Catal. 2012, 55, 1181-1200.

(84) Leech, D.; Kavanagh, P.; Schuhmann, W. Enzymatic Fuel Cells: Recent Progress. Electrochim. Acta 2012, 84, 223-234.

(85) Zhou, M.; Wang, J. Biofuel Cells for Self-Powered Electrochemical Biosensing and Logic Biosensing: A Review. Carbon 2012, 24, 197-209.

(86) Zhou, M. Recent Progress on the Development of Biofuel Cells for Self-Powered Electrochemical Biosensing and Logic Biosensing: A Review. Electroanalysis 2015, 27, 1786-1810.

(87) Zhao, C.-e.; Gai, P.; Song, R.; Chen, Y.; Zhang, J.; Zhu, J.-J. Nanostructured Material-Based Biofuel Cells: Recent Advances and Future Prospects. Chem. Soc. Rev. 2017, 46, 1545-1564.

(88) Mazurenko, I.; Wang, X.; de Poulpiquet, A.; Lojou, E. $\mathrm{H}_{2} / \mathrm{O}_{2}$ Enzymatic Fuel Cells: From Proof-of-Concept to Powerful Devices. Sustainable Energy Fuels 2017, 1, 1475-1501.

(89) Cosnier, S.; Holzinger, M.; Le Goff, A. Recent Advances in Carbon Nanotube-Based Enzymatic Fuel Cells. Front. Bioeng. Biotechnol. 2014, 2, 45.

(90) de Poulpiquet, A.; Ciaccafava, A.; Lojou, E. New Trends in Enzyme Immobilization at Nanostructured Interfaces for Efficient Electrocatalysis in Biofuel Cells. Electrochim. Acta 2014, 126, 104-114.

(91) Holade, Y.; Tingry, S.; Servat, K.; Napporn, T.; Cornu, D.; Kokoh, K. Nanostructured Inorganic 
Materials at Work in Electrochemical Sensing and Biofuel Cells. Catalysts 2017, 7, 31.

(92) Wen, D.; Eychmüller, A. Enzymatic Biofuel Cells on Porous Nanostructures. Small 2016, 12, 4649-4661.

(93) Karimi, A.; Othman, A.; Uzunoglu, A.; Stanciu, L.; Andreescu, S. Graphene Based Enzymatic Bioelectrodes and Biofuel Cells. Nanoscale 2015, 7, 6909-6923.

(94) Liu, Y.; Du, Y.; Li, C. M. Direct Electrochemistry Based Biosensors and Biofuel Cells Enabled with Nanostructured Materials. Electroanalysis 2013, 25, 815-831.

(95) Qiu, H. J.; Guan, Y.; Luo, P.; Wang, Y. Recent Advance in Fabricating Monolithic 3D Porous Graphene and Their Applications in Biosensing and Biofuel Cells. Biosens. Bioelectron. 2017, 89, 85-95.

(96) Yang, X.-Y.; Tian, G.; Jiang, N.; Su, B.-L. Immobilization Technology: A Sustainable Solution for Biofuel Cell Design. Energy Environ. Sci. 2012, 5, 5540-5563.

(97) Ammam, M. Electrochemical and Electrophoretic Deposition of Enzymes: Principles, Differences and Application in Miniaturized Biosensor and Biofuel Cell Electrodes. Biosens. Bioelectron. 2014, 58, 121-131.

(98) Yates, N. D. J.; Fascione, M. A.; Parkin, A. Methodologies for "Wiring" Redox Proteins/Enzymes to Electrode Surfaces. Chem. Eur. J. 2018, 24, 12164-12182.

(99) Le Goff, A.; Holzinger, M.; Cosnier, S. Recent Progress in Oxygen-Reducing Laccase Biocathodes for Enzymatic Biofuel Cells. Cell Mol Life Sci. 2015, 72, 941-952.

(100) Meredith, M. T.; Minteer, S. D. Biofuel Cells: Enhanced Enzymatic Bioelectrocatalysis. Annu. Rev. Anal. Chem. 2012, 5, 157-179.

(101) Betancor, L.; Johnson, G. R.; Luckarift, H. R. Stabilized Laccases as Heterogeneous Bioelectrocatalysts. ChemCatChem 2013, 5, 46-60.

(102) So, K.; Sakai, K.; Kano, K. Gas Diffusion Bioelectrodes. Curr. Opin. Electrochem. 2017, 5, 173-182.

(103) Catalano, P. N.; Wolosiuk, A.; Soler-Illia, G. J. A. A.; Bellino, M. G. Wired Enzymes in Mesoporous Materials: A Benchmark for Fabricating Biofuel Cells. Bioelectrochem. 2015, 106, 14-21.

(104) Mostafavi, S. M. 3D Graphene Biocatalysts for Development of Enzymatic Biofuel Cells: A Short Review. J. Nanoanalysis 2015, 2, 57-62.

(105) Milton, R. D.; Wang, T.; Knoche, K. L.; Minteer, S. D. Tailoring Biointerfaces for Electrocatalysis. Langmuir 2016, 32, 2291-2301.

(106) Katz, E. Magneto-Switchable Electrodes and Electrochemical Systems. Electroanalysis 2016, 28, 904-919.

(107) Desmet, C.; Marquette, C. A.; Blum, L. J.; Doumèche, B. Paper Electrodes for Bioelectrochemistry: Biosensors and Biofuel Cells. Biosens. Bioelectron. 2016, 76, 145-163.

(108) Xiao, X.; Si, P.; Magner, E. An Overview of Dealloyed Nanoporous Gold in Bioelectrochemistry. Bioelectrochem. 2016, 109, 117-126.

(109) Tello, A.; Cao, R.; Marchant, M. J.; Gomez, H. Conformational Changes of Enzymes and Aptamers Immobilized on Electrodes. Bioconjugate Chem. 2016, 27, 2581-2591.

(110) Küchler, A.; Yoshimoto, M.; Luginbühl, S.; Mavelli, F.; Walde, P. Enzymatic Reactions in Confined Environments. Nat. Nanotechnol. 2016, 11, 409.

(111)Huong Le, T. X.; Bechelany, M.; Cretin, M. Carbon Felt Based-Electrodes for Energy and 
Environmental Applications: A Review. Carbon 2017, 122, 564-591.

(112) Kim, J.; Kumar, R.; Bandodkar, A. J.; Wang, J. Advanced Materials for Printed Wearable Electrochemical Devices: A Review. Adv. Electron. Mater. 2017, 3, 1600260.

(113) Stine, K. J. Enzyme Immobilization on Nanoporous Gold: A Review. Biochem. Insights 2017, 10, 1178626417748607.

(114) Mazurenko, I.; de Poulpiquet, A.; Lojou, E. Recent Developments in High Surface Area Bioelectrodes for Enzymatic Fuel Cells. Curr. Opin. Electrochem. 2017, 5, 74-84.

(115) Adeel, M.; Bilal, M.; Rasheed, T.; Sharma, A.; Iqbal, H. M. N. Graphene and Graphene Oxide: Functionalization and Nano-Bio-Catalytic System for Enzyme. Int. J. Biol. Macromol. 2018, $120,1430-1440$.

(116) Le Goff, A.; Holzinger, M. Molecular Engineering of the Bio/Nano-Interface for Enzymatic Electrocatalysis in Fuel Cells. Sustainable Energy Fuels 2018, 2, 2555-2566.

(117) Gross, A. J.; Holzinger, M.; Cosnier, S. Buckypaper Bioelectrodes: Emerging Materials for Implantable and Wearable Biofuel Cells. Energy Environ. Sci. 2018, 11, 1670-1687.

(118) Janczuk-Richter, M.; Marinović, I.; Niedziółka-Jönsson, J.; Szot-Karpińska, K. Recent Applications of Bacteriophage-Based Electrodes: A Mini-Review. Electrochem. Commun. 2019, 99, $11-15$.

(119) Casado, N.; Hernández, G.; Sardon, H.; Mecerreyes, D. Current Trends in Redox Polymers for Energy and Medicine. Prog. Polym. Sci. 2016, 52, 107-135.

(120) Ruff, A. Redox Polymers in Bioelectrochemistry: Common Playgrounds and Novel Concepts. Curr. Opin. Electrochem. 2017, 5, 66-73.

(121) Horst, A. E. W.; Mangold, K.-M.; Holtmann, D. Application of Gas Diffusion Electrodes in Bioelectrochemical Syntheses and Energy Conversion. Biotechnol. Bioeng. 2016, 113, 260-267.

(122) Renner, J. N.; Minteer, S. D. The Use of Engineered Protein Materials in Electrochemical Devices. Exp. Biol. Med. 2016, 241, 980-985.

(123) Minteer, S. D. Oxidative Bioelectrocatalysis: From Natural Metabolic Pathways to Synthetic Metabolons and Minimal Enzyme Cascades. Biochim. Biophys. Acta Bioenerg. 2016, 1857, 621-624.

(124) Macazo, F. C.; Minteer, S. D. Enzyme Cascades in Biofuel Cells. Curr. Opin. Electrochem. 2017, 5, 114-120.

(125) Milton, R. D.; Minteer, S. D. Direct Enzymatic Bioelectrocatalysis: Differentiating between Myth and Reality. J. R. Soc. interface 2017, 14, 20170253.

(126) Rajendran, L.; Kirthiga, M.; Laborda, E. Mathematical Modeling of Nonlinear Reaction-Diffusion Processes in Enzymatic Biofuel Cells. Curr. Opin. Electrochem. 2017, 1, 121-132.

(127) Bostick, C. D.; Mukhopadhyay, S.; Pecht, I.; Sheves, M.; Cahen, D.; Lederman, D. Protein Bioelectronics: A Review of What We Do and Do Not Know. Rep. Prog. Phys. 2018, 81, 026601.

(128) Hitaishi, V.; Clement, R.; Bourassin, N.; Baaden, M.; de Poulpiquet, A.; Sacquin-Mora, S.; Ciaccafava, A.; Lojou, E. Controlling Redox Enzyme Orientation at Planar Electrodes. Catalysts 2018, 8, 192.

(129) Jenner, L. P.; Butt, J. N. Electrochemistry of Surface-Confined Enzymes: Inspiration, Insight and Opportunity for Sustainable Biotechnology. Curr. Opin. Electrochem. 2018, 8, 81-88.

(130) Ma, S.; Ludwig, R. Direct Electron Transfer of Enzymes Facilitated by Cytochromes. ChemElectroChem 2019, 6, 958-975. 
(131) Yamashita, Y.; Lee, I.; Loew, N.; Sode, K. Direct Electron Transfer (DET) Mechanism of FAD Dependent Dehydrogenase Complexes $\sim$ From the Elucidation of Intra- and Inter-Molecular Electron Transfer Pathway to the Construction of Engineered DET Enzyme Complexes . Curr. Opin. Electrochem. 2018, 12, 92-100.

(132) Bollella, P.; Gorton, L.; Antiochia, R. Direct Electron Transfer of Dehydrogenases for Development of 3rd Generation Biosensors and Enzymatic Fuel Cells. Sensors 2018, 18, 1319.

(133) Armstrong, F. A.; Evans, R. M.; Hexter, S. V.; Murphy, B. J.; Roessler, M. M.; Wulff, P. Guiding Principles of Hydrogenase Catalysis Instigated and Clarified by Protein Film Electrochemistry. Acc. Chem. Res. 2016, 49, 884-892.

(134) Sensi, M.; del Barrio, M.; Baffert, C.; Fourmond, V.; Léger, C. New Perspectives in Hydrogenase Direct Electrochemistry. Curr. Opin. Electrochem. 2017, 5, 135-145.

(135) Shleev, S.; Andoralov, V.; Pankratov, D.; Falk, M.; Aleksejeva, O.; Blum, Z. Oxygen Electroreduction Versus Bioelectroreduction: Direct Electron Transfer Approach. Electroanalysis 2016, 28, 2270-2287.

(136) Mate, D. M.; Alcalde, M. Laccase: A Multi-Purpose Biocatalyst at the Forefront of Biotechnology. Microb. Biotechnol. 2017, 10, 1457-1467.

(137) Mano, N.; de Poulpiquet, A. $\mathrm{O}_{2}$ Reduction in Enzymatic Biofuel Cells. Chem. Rev. 2017, 118, 2392-2468.

(138) Zhang, Y.; Lv, Z.; Zhou, J.; Xin, F.; Ma, J.; Wu, H.; Fang, Y.; Jiang, M.; Dong, W. Application of Eukaryotic and Prokaryotic Laccases in Biosensor and Biofuel Cells: Recent Advances and Electrochemical Aspects. Appl. Microbiol. Biotechnol. 2018, 102, 10409-10423.

(139) Sode, K.; Yamazaki, T.; Lee, I.; Hanashi, T.; Tsugawa, W. BioCapacitor: A Novel Principle for Biosensors. Biosens. Bioelectron. 2016, 76, 20-28.

(140) Shleev, S.; González-Arribas, E.; Falk, M. Biosupercapacitors. Curr. Opin. Electrochem. 2017, 5, 226-233.

(141) Yang, Y.; Liu, T.; Tao, K.; Chang, H. Generating Electricity on Chips: Microfluidic Biofuel Cells in Perspective. Ind. Eng. Chem. Res. 2018, 57, 2746-2758.

(142) Pankratov, D.; González-Arribas, E.; Blum, Z.; Shleev, S. Tear Based Bioelectronics. Electroanalysis 2016, 28, 1250-1266.

(143) Shi, B.; Li, Z.; Fan, Y. Implantable Energy-Harvesting Devices. Adv. Mater. 2018, 0, 1801511

(144) Kim, J.; Jeerapan, I.; Sempionatto, J. R.; Barfidokht, A.; Mishra, R. K.; Campbell, A. S.; Hubble, L. J.; Wang, J. Wearable Bioelectronics: Enzyme-Based Body-Worn Electronic Devices. Acc. Chem. Res. 2018, 51, 2820-2828.

(145) Huang, X.; Zhang, L.; Zhang, Z.; Guo, S.; Shang, H.; Li, Y.; Liu, J. Wearable Biofuel Cells Based on the Classification of Enzyme for High Power Outputs and Lifetimes. Biosens. Bioelectron. 2019, 124-125, 40-52.

(146) Dagdeviren, C.; Li, Z.; Wang, Z. L. Energy Harvesting from the Animal/Human Body for Self-Powered Electronics. Annu. Rev. Biomed. Eng. 2017, 19, 85-108.

(147) Fu, L.; Liu, J.; Hu, Z.; Zhou, M. Recent Advances in the Construction of Biofuel Cells Based Self-powered Electrochemical Biosensors: A Review. Electroanalysis 2018, 30, 2535-2550.

(148) Niitsu, K. Energy-Autonomous Biosensing Platform Using Supply-Sensing CMOS 
Integrated Sensor and Biofuel Cell for Next-Generation Healthcare Internet of Things. Jpn. J. Appl. Phys. 2018, 57, 1002A1005.

(149) Conzuelo, F.; Ruff, A.; Schuhmann, W. Self-Powered Bioelectrochemical Devices. Curr. Opin. Electrochem. 2018, 156-163.

(150) Gonzalez-Solino, C.; Lorenzo, D. M. Enzymatic Fuel Cells: Towards Self-Powered Implantable and Wearable Diagnostics. Biosensors 2018, 8.

(151) Grattieri, M.; Minteer, S. D. Self-Powered Biosensors. ACS Sens. 2018, 3, 44-53.

(152) Abdellaoui, S.; Macazo Florika, C.; Cai, R.; De Lacey Antonio, L.; Pita, M.; Minteer Shelley, D. Enzymatic Electrosynthesis of Alkanes by Bioelectrocatalytic Decarbonylation of Fatty Aldehydes. Angew. Chem. Int. Ed. 2017, 57, 2404-2408.

(153) Milton, R. D.; Cai, R.; Abdellaoui, S.; Leech, D.; De Lacey, A. L.; Pita, M.; Minteer, S. D. Bioelectrochemical Haber-Bosch Process: An Ammonia-Producing $\mathrm{H}_{2} / \mathrm{N}_{2}$ Fuel Cell. Angew. Chem. Int. Ed. 2017, 56, 2680-2683.

(154) Gentil, S.; Che Mansor, S. M.; Jamet, H.; Cosnier, S.; Cavazza, C.; Le Goff, A. Oriented Immobilization of [NiFeSe] Hydrogenases on Covalently and Noncovalently Functionalized Carbon Nanotubes for H2/Air Enzymatic Fuel Cells. ACS Catal. 2018, 8, 3957-3964.

(155) Palmore, G. T. R.; Bertschy, H.; Bergens, S. H.; Whitesides, G. M. A Methanol/Dioxygen Biofuel Cell that Uses $\mathrm{NAD}(+)$-Dependent Dehydrogenases as Catalysts: Application of an Electro-Enzymatic Method to Regenerate Nicotinamide Adenine Dinucleotide at Low Overpotentials. $J$. Electroanal. Chem. 1998, 443, 155-161.

(156) Arechederra, R. L.; Minteer, S. D. Complete Oxidation of Glycerol in an Enzymatic Biofuel Cell. Fuel Cells 2009, 9, 63-69.

(157) Sokic-Lazic, D.; Minteer, S. D. Pyruvate/Air Enzymatic Biofuel Cell Capable of Complete Oxidation. Electrochem. Solid-State Lett. 2009, 12, F26-F28.

(158) Li, L.; Liang, B.; Li, F.; Shi, J.; Mascini, M.; Lang, Q.; Liu, A. Co-Immobilization of Glucose Oxidase and Xylose Dehydrogenase Displayed Whole Cell on Multiwalled Carbon Nanotube Nanocomposite Films Modified Electrode for Simultaneous Voltammetric Detection of $D$-Glucose and D-Xylose. Biosens. Bioelectron. 2013, 42, 156-162.

(159) Zhu, Z.; Wang, Y.; Minteer, S. D.; Percival Zhang, Y. H. Maltodextrin-Powered Enzymatic Fuel Cell Through a Non-Natural Enzymatic Pathway. J. Power Sources 2011, 196, 7505-7509.

(160) Sokic-Lazic, D.; Minteer, S. D. Citric Acid Cycle Biomimic on a Carbon Electrode. Biosens. Bioelectron. 2008, 24, 939-944.

(161) Akers, N. L.; Moore, C. M.; Minteer, S. D. Development of Alcohol $/ \mathrm{O}_{2}$ Biofuel Cells Using Salt-Extracted Tetrabutylammonium Bromide/Nafion Membranes to Immobilize Dehydrogenase Enzymes. Electrochim. Acta 2005, 50, 2521-2525.

(162) Arechederra, R. L.; Boehm, K.; Minteer, S. D. Mitochondrial Bioelectrocatalysis for Biofuel Cell Applications. Electrochim. Acta 2009, 54, 7268-7273.

(163) Addo, P. K.; Arechederra, R. L.; Minteer, S. D. Evaluating Enzyme Cascades for Methanol/Air Biofuel Cells Based on NAD'-Dependent Enzymes. Electroanal 2010, 22, 807-812.

(164) Kim, Y. H.; Campbell, E.; Yu, J.; Minteer, S. D.; Banta, S. Complete Oxidation of Methanol in Biobattery Devices Using a Hydrogel Created from Three Modified Dehydrogenases. Angew. Chem. Int. Ed. 2013, 52, 1437-1440. 
Nitride-Graphene for the Selective Electro-Oxidation of Glycerol in Alkaline Solution. ACS Catal. 2015, 5, 3174-3180.

(166) Hickey, D. P.; McCammant, M. S.; Giroud, F.; Sigman, M. S.; Minteer, S. D. Hybrid Enzymatic and Organic Electrocatalytic Cascade for the Complete Oxidation of Glycerol. J. Am. Chem. Soc. 2014, 136, 15917-15920.

(167) Zhu, Z.; Ma, C.; Percival Zhang, Y. H. Co-Utilization of Mixed Sugars in an Enzymatic Fuel Cell Based on an in vitro Enzymatic Pathway. Electrochim. Acta 2018, 263, 184-191.

(168) Cosnier, S.; Shan, D.; Ding, S. N. An Easy Compartment-Less Biofuel Cell Construction Based on the Physical Co-Inclusion of Enzyme and Mediator Redox within Pressed Graphite Discs. Electrochem. Commun. 2010, 12, 266-269.

(169) Tasca, F.; Gorton, L.; Kujawa, M.; Patel, I.; Harreither, W.; Peterbauer, C. K.; Ludwig, R.; Nöll, G. Increasing the Coulombic Efficiency of Glucose Biofuel Cell Anodes by Combination of Redox Enzymes. Biosens. Bioelectron. 2010, 25, 1710-1716.

(170) Xu, S.; Minteer, S. D. Enzymatic Biofuel Cell for Oxidation of Glucose to $\mathrm{CO}_{2}$. ACS Catal. 2011, 2, 91-94.

(171) Zhu, Z. G.; Sun, F. F.; Zhang, X. Z.; Zhang, Y. H. P. Deep Oxidation of Glucose in Enzymatic Fuel Cells Through a Synthetic Enzymatic Pathway Containing a Cascade of Two Thermostable Dehydrogenases. Biosens. Bioelectron. 2012, 36, 110-115.

(172) Wu, R.; Ma, C.; Zhang, Y. H. P.; Zhu, Z. Complete Oxidation of Xylose for Bioelectricity Generation by Reconstructing a Bacterial Xylose Utilization Pathway in vitro. ChemCatChem 2018, 10, $1-7$.

(173) Szczupak, A.; Aizik, D.; Moraïs, S.; Vazana, Y.; Barak, Y.; Bayer, E. A.; Alfonta, L. The Electrosome: A Surface-Displayed Enzymatic Cascade in a Biofuel Cell's Anode and a High-Density Surface-Displayed Biocathodic Enzyme. Nanomaterials 2017, 7, 153.

(174) Wheeldon, I.; Minteer, S.; Banta, S.; Barton, S.; Atanassov, P.; Sigman, M. Substrate Channelling as an Approach to Cascade Reactions. Nat. Chem. 2016, 8, 299-309.

(175) Liu, Y. C.; Hickey, D. P.; Guo, J. Y.; Earl, E.; Abdellaoui, S.; Milton, R. D.; Sigman, M. S.; Minteer, S. D.; Barton, S. C. Substrate Channeling in an Artificial Metabolon: A Molecular Dynamics Blueprint for an Experimental Peptide Bridge. ACS Catal. 2017, 7, 2486-2493.

(176) Xia, L.; Nguyen, K. V.; Holade, Y.; Han, H.; Dooley, K.; Atanassov, P.; Banta, S.; Minteer, S. D. Improving the Performance of Methanol Biofuel Cells Utilizing an Enzyme Cascade Bioanode with DNA-Bridged Substrate Channeling. ACS Energy Lett. 2017, 2, 1435-1438.

(177) Moehlenbrock, M. J.; Toby, T. K.; Waheed, A.; Minteer, S. D. Metabolon Catalyzed Pyruvate/Air Biofuel Cell. J. Am. Chem. Soc. 2010, 132, 6288-6289.

(178) de Souza, J. C. P.; Silva, W. O.; Lima, F. H. B.; Crespilho, F. N. Enzyme Activity Evaluation by Differential Electrochemical Mass Spectrometry. Chem. Commun. 2017, 53, 8400-8402.

(179) Zhao, F.; Slade, R. C. T.; Varcoe, J. R. Techniques for the study and development of microbial fuel cells: an electrochemical perspective. Chem. Soc. Rev. 2009, 38, 1926-1939.

(180) Trifonov, A.; Tel-Vered, R.; Fadeev, M.; Willner, I. Electrically Contacted Bienzyme-Functionalized Mesoporous Carbon Nanoparticle Electrodes: Applications for the Development of Dual Amperometric Biosensors and Multifuel-Driven Biofuel Cells. Adv. Energy 
Mater. 2015, 5, 1401853.

(181) Meredith, M. T.; Minson, M.; Hickey, D.; Artyushkova, K.; Glatzhofer, D. T.; Minteer, S. D. Anthracene-Modified Multi-Walled Carbon Nanotubes as Direct Electron Transfer Scaffolds for Enzymatic Oxygen Reduction. ACS Catal. 2011, 1, 1683-1690.

(182) Xu, L.; Armstrong, F. A. Pushing the Limits for Enzyme-Based Membrane-Less Hydrogen Fuel Cells - Achieving Useful Power and Stability. RSC Adv. 2015, 5, 3649-3656.

(183) Kizling, M.; Dzwonek, M.; Olszewski, B.; Bącal, P.; Tymecki, Ł.; Więckowska, A.; Stolarczyk, K.; Bilewicz, R. Reticulated Vitreous Carbon as a Scaffold for Enzymatic Fuel Cell Designing. Biosens. Bioelectron. 2017, 95, 1-7.

(184) Fadzillah, D. M.; Kamarudin, S. K.; Zainoodin, M. A.; Masdar, M. S. Critical Challenges in the System Development of Direct Alcohol Fuel Cells as Portable Power Supplies: An Overview. Int. J. Hydrogen Energy 2019, 44, 3031-3054.

(185) Gross, A. J.; Chen, X.; Giroud, F.; Abreu, C.; Le Goff, A.; Holzinger, M.; Cosnier, S. A High Power Buckypaper Biofuel Cell: Exploiting 1,10-Phenanthroline-5,6-Dione with FAD-Dependent Dehydrogenase for Catalytically-Powerful Glucose Oxidation. ACS Catal. 2017, 7, 4408-4416.

(186) Campbell, A. S.; Jeong, Y. J.; Geier, S. M.; Koepsel, R. R.; Russell, A. J.; Islam, M. F. Membrane/Mediator-Free Rechargeable Enzymatic Biofuel Cell Utilizing Graphene/Single-Wall Carbon Nanotube Cogel Electrodes. ACS Appl. Mater. Interfaces 2015, 7, 4056-4065.

(187) Shao, B. B.; Liu, Z. F.; Zeng, G. M.; Liu, Y.; Yang, X.; Zhou, C. Y.; Chen, M.; Liu, Y. J.; Jiang, Y. L.; Yan, M. Immobilization of Laccase on Hollow Mesoporous Carbon Nanospheres: Noteworthy Immobilization, Excellent Stability and Efficacious for Antibiotic Contaminants Removal. J. Hazard. Mater. 2019, 362, 318-326.

(188) Sadeghi, S.; Fooladi, E.; Malekaneh, M. A New Amperometric Biosensor Based on $\mathrm{Fe}_{3} \mathrm{O}_{4} /$ Polyaniline/Laccase/Chitosan Biocomposite-Modified Carbon Paste Electrode for Determination of Catechol in Tea Leaves. Appl. Biochem. Biotechnol. 2015, 175, 1603-1616.

(189) Itoh, T.; Shibuya, Y.; Yamaguchi, A.; Hoshikawa, Y.; Tanaike, O.; Tsunoda, T.; Hanaoka, T. A.; Hamakawa, S.; Mizukami, F.; Hayashi, A.et al. High-Performance Bioelectrocatalysts Created by Immobilization of an Enzyme into Carbon-Coated Composite Membranes with Nano-Tailored Structures. J. Mater. Chem. A 2017, 5, 20244-20251.

(190) Campbell, A. S.; Jose, M. V.; Marx, S.; Cornelius, S.; Koepsel, R. R.; Islam, M. F.; Russell, A. J. Improved Power Density of an Enzymatic Biofuel Cell with Fibrous Supports of High Curvature. RSC Adv. 2016, 6, 10150-10158.

(191) Shukla, A.; Suresh, P.; Berchmans, S.; Rajendran, A. Biological Fuel Cells and Their Applications. Curr. Sci. 2004, 87, 455-468.

(192) Michaelis, L.; Menten, M. L. The Kinetics of the Inversion Effect. Biochem. Z. 1913, 49, 333-369.

(193) Bisswanger, H. Enzyme Assays. Perspect. Sci. 2014, 1, 41-55.

(194) Koshland, D. E. The Application and Usefulness of the Ratio $\mathrm{k}_{\mathrm{cat}} / \mathrm{K}_{\mathrm{M}}$. Bioorg. Chem. 2002, 30, 211-213.

(195) Laidler, K. J. Symbolism and Terminology in Chemical Kinetics. Pure Appl. Chem. 1981, 53, 753-771.

(196) Güven, G.; Prodanovic, R.; Schwaneberg, U. Protein Engineering-An Option for Enzymatic 
Biofuel Cell Design. Electroanalysis 2010, 22, 765-775.

(197) Zhu, Z.; Momeu, C.; Zakhartsev, M.; Schwaneberg, U. Making Glucose Oxidase Fit for Biofuel Cell Applications by Directed Protein Evolution. Biosens. Bioelectron. 2006, 21, 2046-2051.

(198) Zhu, Z; Wang, M.; Gautam, A.; Nazor, J.; Momeu, C.; Prodanovic, R.; Schwaneberg, U.

Directed Evolution of Glucose Oxidase from Aspergillus niger for Ferrocenemethanol-Mediated Electron Transfer. Biotechnol. J. 2007, 2, 241-248.

(199) Mano, N. A $280 \mu \mathrm{W} \mathrm{cm}{ }^{-2}$ Biofuel Cell Operating at Low Glucose Concentration. Chem. Commun. 2008, 0, 2221-2223.

(200) Courjean, O.; Mano, N. Recombinant Glucose Oxidase from Penicillium amagasakiense for Efficient Bioelectrochemical Applications in Physiological Conditions. J. Biotechnol. 2011, 151, 122-129.

(201) Prévoteau, A.; Courjean, O.; Mano, N. Deglycosylation of Glucose Oxidase to Improve Biosensors and Biofuel Cells. Electrochem. Commun. 2010, 12, 213-215.

(202) Marcus, R. A. Electron Transfer Reactions in Chemistry: Theory and Experiment (Nobel Lecture). Angew. Chem. Int. Ed. 1993, 32, 1111-1121.

(203) Mayo, S. L.; Ellis, W. R.; Crutchley, R. J.; Gray, H. B. Long-Range Electron Transfer in Heme Proteins. Science 1986, 233, 948-952.

(204) Page, C. C.; Moser, C. C.; Chen, X.; Dutton, P. L. Natural Engineering Principles of Electron Tunnelling in Biological Oxidation-Reduction. Nature 1999, 402, 47-52.

(205) Wang, J. Electrochemical Glucose Biosensors. Chem. Rev. 2008, 108, 814-825.

(206) Sucheta, A.; Cammack, R.; Weiner, J.; Armstrong, F. A. Reversible Electrochemistry of Fumarate Reductase Immobilized on an Electrode Surface. Direct Voltammetric Observations of Redox Centers and Their Participation in Rapid Catalytic Electron Transport. Biochemistry 1993, 32, 5455-5465.

(207) Armstrong, F. A. Recent Developments in Dynamic Electrochemical Studies of Adsorbed Enzymes and Their Active Sites. Curr. Opin. Chem. Biol. 2005, 9, 110-117.

(208) Léger, C.; Bertrand, P. Direct Electrochemistry of Redox Enzymes as a Tool for Mechanistic Studies. Chem. Rev. 2008, 108, 2379-2438.

(209) Laviron, E. General Expression of the Linear Potential Sweep Voltammogram in the Case of Diffusionless Electrochemical Systems. J. Electroanal. Chem. Interfacial Electrochem. 1979, 101, 19-28.

(210) Léger, C.; Jones, A. K.; Albracht, S. P. J.; Armstrong, F. A. Effect of a Dispersion of Interfacial Electron Transfer Rates on Steady State Catalytic Electron Transport in [NiFe]-hydrogenase and Other Enzymes. J. Phys. Chem. B 2002, 106, 13058-13063.

(211) Zayats, M.; Katz, E.; Willner, I. Electrical Contacting of Flavoenzymes and NAD(P)+-Dependent Enzymes by Reconstitution and Affinity Interactions on Phenylboronic Acid Monolayers Associated with Au-Electrodes. J. Am. Chem. Soc. 2002, 124, 14724-14735.

(212) Agip, A.-N. A.; Blaza, J. N.; Fedor, J. G.; Hirst, J. Mammalian Respiratory Complex I Through the Lens of Cryo-EM. Annu. Rev. Biophys. 2019, 48, 165-184.

(213) Burrows, A. L.; Guo, L. H.; Hill, H. A.; McLendon, G.; Sherman, F. Direct Electrochemistry of Proteins. Investigations of Yeast Cytochrome $\mathrm{c}$ Mutants and Their Complexes with Cytochrome $\mathrm{b}_{5}$ 
Eur. J. Biochem. 1991, 202, 543-549.

(214) Ludwig, R.; Harreither, W.; Tasca, F.; Gorton, L. Cellobiose Dehydrogenase: A Versatile Catalyst for Electrochemical Applications. ChemPhysChem 2010, 11, 2674-2697.

(215) Ludwig, R.; Ortiz, R.; Schulz, C.; Harreither, W.; Sygmund, C.; Gorton, L. Cellobiose Dehydrogenase Modified Electrodes: Advances by Materials Science and Biochemical Engineering. Anal. Bioanal. Chem. 2013, 405, 3637-3658.

(216) Ameyama, M.; Shinagawa, E.; Matsushita, K.; Adachi, O. D-Fructose Dehydrogenase of Gluconobacter Industrius: Purification, Characterization, and Application to Enzymatic Microdetermination of $D$-Fructose. J. Bacteriol. 1981, 145, 814-823.

(217) Xia, H.-q.; Hibino, Y.; Kitazumi, Y.; Shirai, O.; Kano, K. Interaction between D-Fructose Dehydrogenase and Methoxy-Substituent-Functionalized Carbon Surface to Increase Productive Orientations. Electrochim. Acta 2016, 218, 41-46.

(218) Kizling, M.; Bilewicz, R. Fructose Dehydrogenase Electron Transfer Pathway in Bioelectrocatalytic Reactions. ChemElectroChem 2017, 5, 166-174.

(219) Yamashita, Y.; Ferri, S.; Huynh, M. L.; Shimizu, H.; Yamaoka, H.; Sode, K. Direct Electron Transfer Type Disposable Sensor Strip for Glucose Sensing Employing an Engineered FAD Glucose Dehydrogenase. Enzyme Microb. Technol. 2013, 52, 123-128.

(220) Shiota, M.; Yamazaki, T.; Yoshimatsu, K.; Kojima, K.; Tsugawa, W.; Ferri, S.; Sode, K. An Fe-S Cluster in the Conserved Cys-Rich Region in the Catalytic Subunit of FAD-Dependent Dehydrogenase Complexes. Bioelectrochemistry 2016, 112, 178-183.

(221) Babanova, S.; Matanovic, I.; Chavez, M. S.; Atanassov, P. Role of Quinones in Electron Transfer of PQQ-Glucose Dehydrogenase Anodes-Mediation or Orientation Effect. J. Am. Chem. Soc. 2015, 137, 7754-7762.

(222) Ivnitski, D.; Atanassov, P.; Apblett, C. Direct Bioelectrocatalysis of PQQ-Dependent Glucose Dehydrogenase. Electroanalysis 2007, 19, 1562-1568.

(223) Torimura, M.; Kano, K.; Ikeda, T.; Ueda, T. Spectroelectrochemical Characterization of Quinohemoprotein Alcohol Dehydrogenase from Gluconobacter suboxydans. Chem. Lett. 1997, 26, 525-526.

(224) Treu, B. L.; Minteer, S. D. Isolation and Purification of PQQ-Dependent Lactate Dehydrogenase from Gluconobacter and Use for Direct Electron Transfer at Carbon and Gold Electrodes. Bioelectrochemistry 2008, 74, 73-77.

(225) Laurinavicius, V.; Razumiene, J.; Ramanavicius, A.; Ryabov, A. D. Wiring of PQQ-Dehydrogenases. Biosens. Bioelectron. 2004, 20, 1217-1222.

(226) Okuda, J.; Sode, K. PQQ Glucose Dehydrogenase with Novel Electron Transfer Ability. Biochem. Biophys. Res. Commun. 2004, 314, 793-797.

(227) Ito, K.; Okuda-Shimazaki, J.; Mori, K.; Kojima, K.; Tsugawa, W.; Ikebukuro, K.; Lin, C.-E.; La Belle, J.; Yoshida, H.; Sode, K. Designer Fungus FAD Glucose Dehydrogenase Capable of Direct Electron Transfer. Biosens. Bioelectron. 2019, 123, 114-123.

(228) Lubitz, W.; Ogata, H.; Rüdiger, O.; Reijerse, E. Hydrogenases. Chem. Rev. 2014, 114, 4081-4148.

(229) Lojou, É.; Luo, X.; Brugna, M.; Candoni, N.; Dementin, S.; Giudici-Orticoni, M. T. Biocatalysts for Fuel Cells: Efficient Hydrogenase Orientation for $\mathrm{H}_{2}$ Oxidation at Electrodes 
Mmodified with Carbon Nanotubes. J. Biol. Inorg. Chem. 2008, 13, 1157-1167.

(230) Gutiérrez-Sánchez, C.; Pita, M.; Vaz-Domínguez, C.; Shleev, S.; De Lacey, A. L. Gold Nanoparticles as Electronic Bridges for Laccase-Based Biocathodes. J. Am. Chem. Soc. 2012, 134, 17212-17220.

(231) Solomon, E. I.; Augustine, A. J.; Yoon, J. $\mathrm{O}_{2}$ Reduction to $\mathrm{H}_{2} \mathrm{O}$ by the multicopper oxidases. Dalton Trans. 2008, 0, 3921-3932.

(232) Quintanar, L.; Stoj, C.; Taylor, A. B.; Hart, P. J.; Kosman, D. J.; Solomon, E. I. Shall We Dance? How A Multicopper Oxidase Chooses Its Electron Transfer Partner. Acc. Chem. Res. 2007, 40, 445-452.

(233) Sakurai, T.; Kataoka, K. Basic and Applied Features of Multicopper Oxidases, CueO, Bilirubin Oxidase, and Laccase. Chem. Rec. 2007, 7, 220-229.

(234) Gutierrez-Sanchez, C.; Ciaccafava, A.; Blanchard, P. Y.; Monsalve, K.; Giudici-Orticoni, M. T.; Lecomte, S.; Lojou, E. Efficiency of Enzymatic $\mathrm{O}_{2}$ Reduction by Myrothecium Verrucaria Bilirubin Oxidase Probed by Surface Plasmon Resonance, PMIRRAS, and Electrochemistry. ACS Catal. 2016, 6, 5482-5492.

(235) Karyakin, A. A. Principles of Direct (Mediator Free) Bioelectrocatalysis. Bioelectrochemistry 2012, 88, 70-75.

(236) Mazurenko, I.; Monsalve, K.; Rouhana, J.; Parent, P.; Laffon, C.; Goff, A. L.; Szunerits, S.; Boukherroub, R.; Giudici-Orticoni, M.-T.; Mano, N.et al. How the Intricate Interactions between Carbon Nanotubes and Two Bilirubin Oxidases Control Direct and Mediated $\mathrm{O}_{2}$ Reduction. ACS Appl. Mater. Interfaces 2016, 8, 23074-23085.

(237) Oteri, F.; Ciaccafava, A.; Poulpiquet, A. d.; Baaden, M.; Lojou, E.; Sacquin-Mora, S. The Weak, Fluctuating, Dipole Moment of Membrane-Bound Hydrogenase from Aquifex aeolicus Accounts for Its Adaptability to Charged Electrodes. Phys. Chem. Chem. Phys. 2014, 16, 11318-11322.

(238) Pankratov, D.; Sotres, J.; Barrantes, A.; Arnebrant, T.; Shleev, S. Interfacial Behavior and Activity of Laccase and Bilirubin Oxidase on Bare Gold Surfaces. Langmuir 2014, 30, 2943-2951.

(239) Hitaishi, V. P.; Mazurenko, I.; Harb, M.; Clément, R.; Taris, M.; Castano, S.; Duché, D.; Lecomte, S.; Ilbert, M.; de Poulpiquet, A.et al. Electrostatic-Driven Activity, Loading, Dynamics, and Stability of a Redox Enzyme on Functionalized-Gold Electrodes for Bioelectrocatalysis. ACS Catal. 2018, 8, 12004-12014.

(240) Nam, D. H.; Zhang, J. Z.; Andrei, V.; Kornienko, N.; Heidary, N.; Wagner, A.; Nakanishi, K.; Sokol, K. P.; Slater, B.; Zebger, I.et al. Solar Water Splitting with a Hydrogenase Integrated in Photoelectrochemical Tandem Cells. Angew. Chem. Int. Ed. 2018, 57, 10595-10599.

(241) Singh, K.; McArdle, T.; Sullivan, P. R.; Blanford, C. F. Sources of Activity Loss in the Fuel Cell Enzyme Bilirubin Oxidase. Energy Environ. Sci. 2013, 6, 2460-2464.

(242) Ciaccafava, A.; Infossi, P.; Ilbert, M.; Guiral, M.; Lecomte, S.; Giudici-Orticoni, M. T.; Lojou, E. Electrochemistry, AFM, and PM-IRRA Spectroscopy of Immobilized Hydrogenase: Role of a Hydrophobic Helix in Enzyme Orientation for Efficient $\mathrm{H}_{2}$ Oxidation. Angew. Chem. Int. Ed. 2012, 51, 953-956.

(243) Dagys, M.; Laurynėnas, A.; Ratautas, D.; Kulys, J.; Vidžiūnaitė, R.; Talaikis, M.; Niaura, G.; Marcinkevičienė, L.; Meškys, R.; Shleev, S. Oxygen Electroreduction Catalysed by Laccase Wired to Gold Nanoparticles via the Trinuclear Copper Cluster. Energy Environ. Sci. 2017, 10, 498-502. 
(244) Heidary, N.; Utesch, T.; Zerball, M.; Horch, M.; Millo, D.; Fritsch, J.; Lenz, O.; von Klitzing, R.; Hildebrandt, P.; Fischer, A.et al. Orientation-Controlled Electrocatalytic Efficiency of an Adsorbed Oxygen-Tolerant Hydrogenase. PLOS ONE 2015, 10, e0143101.

(245) Olejnik, P.; Palys, B.; Kowalczyk, A.; Nowicka, A. M. Orientation of Laccase on Charged Surfaces. Mediatorless Oxygen Reduction on Amino- and Carboxyl-Ended Ethylphenyl Groups. $J$. Phys. Chem. C 2012, 116, 25911-25918.

(246) Olejnik, P.; Pawłowska, A.; Pałys, B. Application of Polarization Modulated Infrared Reflection Absorption Spectroscopy for Electrocatalytic Activity Studies of Laccase Adsorbed on Modified Gold Electrodes. Electrochim. Acta 2013, 110, 105-111.

(247) Hao, X.; Zhang, J.; Christensen, H. E. M.; Wang, H.; Ulstrup, J. Electrochemical Single-Molecule AFM of the Redox Metalloenzyme Copper Nitrite Reductase in Action. ChemPhysChem 2012, 13, 2919-2924.

(248) Gutiérrez-Sánchez, C.; Olea, D.; Marques, M.; Fernández, V. M.; Pereira, I. A. C.; Vélez, M.; De Lacey, A. L. Oriented Immobilization of a Membrane-Bound Hydrogenase onto an Electrode for Direct Electron Transfer. Langmuir 2011, 27, 6449-6457.

(249) Gutiérrez-Sanz, Ó.; Natale, P.; Márquez, I.; Marques, M. C.; Zacarias, S.; Pita, M.; Pereira, I. A. C.; López-Montero, I.; De Lacey, A. L.; Vélez, M. $\mathrm{H}_{2}$-Fueled ATP Synthesis on an Electrode: Mimicking Cellular Respiration. Angew. Chem. Int. Ed. 2016, 55, 6216-6220.

(250) Harada, H.; Onoda, A.; Uchihashi, T.; Watanabe, H.; Sunagawa, N.; Samejima, M.; Igarashi, K.; Hayashi, T. Interdomain Flip-Flop Motion Visualized in Flavocytochrome Cellobiose Dehydrogenase using High-Speed Atomic Force Microscopy During Catalysis. Chem. Sci. 2017, 8 , 6561-6565.

(251) Cracknell, J. A.; McNamara, T. P.; Lowe, E. D.; Blanford, C. F. Bilirubin Oxidase from Myrothecium verrucaria: X-Ray Determination of the Complete Crystal Structure and a Rational Surface Modification for Enhanced Electrocatalytic $\mathrm{O}_{2}$ Reduction. Dalton Trans. 2011, 40, 6668-6675. (252) Lalaoui, N.; Le Goff, A.; Holzinger, M.; Cosnier, S. Fully Oriented Bilirubin Oxidase on Porphyrin-Functionalized Carbon Nanotube Electrodes for Electrocatalytic Oxygen Reduction. Chem. Eur. J. 2015, 21, 16868-16873.

(253) Vaz-Dominguez, C.; Campuzano, S.; Rüdiger, O.; Pita, M.; Gorbacheva, M.; Shleev, S.; Fernandez, V. M.; De Lacey, A. L. Laccase Electrode for Direct Electrocatalytic Reduction of $\mathrm{O}_{2}$ to $\mathrm{H}_{2} \mathrm{O}$ with High-Operational Stability and Resistance to Chloride Inhibition. Biosens. Bioelectron. 2008, 24, 531-537.

(254) Krishnan, S.; Armstrong, F. A. Order-of-Magnitude Enhancement of an Enzymatic Hydrogen-Air Fuel Cell based on Pyrenyl Carbon Nanostructures. Chem. Sci. 2012, 3, 1015-1023.

(255) Di Bari, C.; Goñi-Urtiaga, A.; Pita, M.; Shleev, S.; Toscano, M. D.; Sainz, R.; De Lacey, A. L. Fabrication of High Surface Area Graphene Electrodes with High Performance Towards Enzymatic Oxygen Reduction. Electrochim. Acta 2016, 191, 500-509.

(256) Gentil, S.; Carriere, M.; Cosnier, S.; Gounel, S.; Mano, N.; Le Goff, A. Direct Electrochemistry of Bilirubin Oxidase from Magnaporthe orizae on Covalently-Functionalized MWCNT for the Design of High-Performance Oxygen-Reducing Biocathodes. Chem. Eur. J. 2018, 24, 8404-8408.

(257) Blanford, C. F.; Heath, R. S.; Armstrong, F. A. A Stable Electrode for High-Potential, 
Electrocatalytic $\mathrm{O}_{2}$ Reduction Based on Rational Attachment of a Blue Copper Oxidase to a Graphite Surface. Chem. Commun. 2007, 0, 1710-1712.

(258) Blanford, C. F.; Foster, C. E.; Heath, R. S.; Armstrong, F. A. Efficient Electrocatalytic Oxygen Reduction by the 'Blue' Copper Oxidase, Laccase, Directly Attached to Chemically Modified Carbons. Faraday Discuss. 2009, 140, 319-335.

(259) Lalaoui, N.; David, R.; Jamet, H.; Holzinger, M.; Le Goff, A.; Cosnier, S. Hosting Adamantane in the Substrate Pocket of Laccase: Direct Bioelectrocatalytic Reduction of $\mathrm{O}_{2}$ on Functionalized Carbon Nanotubes. ACS Catal. 2016, 6, 4259-4264.

(260) Lalaoui, N.; Rousselot-Pailley, P.; Robert, V.; Mekmouche, Y.; Villalonga, R.; Holzinger, M.; Cosnier, S.; Tron, T.; Le Goff, A. Direct Electron Transfer between a Site-Specific Pyrene-Modified Laccase and Carbon Nanotube/Gold Nanoparticle Supramolecular Assemblies for Bioelectrocatalytic Dioxygen Reduction. ACS Catal. 2016, 6, 1894-1900.

(261) Guan, D.; Kurra, Y.; Liu, W.; Chen, Z. A Click Chemistry Approach to Site-Specific Immobilization of a Small Laccase Enables Efficient Direct Electron Transfer in a Biocathode. Chem. Commun. 2015, 51, 2522-2525.

(262) Schlesinger, O.; Pasi, M.; Dandela, R.; Meijler, M. M.; Alfonta, L. Electron Transfer Rate Analysis of a Site-Specifically Wired Copper Oxidase. Phys. Chem. Chem. Phys. 2018, 20, 6159-6166.

(263) Al-Lolage, F. A.; Meneghello, M.; Ma, S.; Ludwig, R.; Bartlett, P. N. A Flexible Method for the Stable, Covalent Immobilization of Enzymes at Electrode Surfaces. ChemElectroChem 2017, 4, 1528-1534.

(264) Kalisz, H. M.; Hecht, H.-J.; Schomburg, D.; Schmid, R. D. Crystallization and Preliminary X-ray Diffraction Studies of a Deglycosylated Glucose Oxidase from Aspergillus niger. J. Mol. Biol. 1990, 213, 207-209.

(265) Courjean, O.; Gao, F.; Mano, N. Deglycosylation of Glucose Oxidase for Direct and Efficient Glucose Electrooxidation on a Glassy Carbon Electrode. Angew. Chem. Int. Ed. 2009, 48, 5897-5899.

(266) Yakovleva, M. E.; Killyéni, A.; Ortiz, R.; Schulz, C.; MacAodha, D.; Conghaile, P. Ó.; Leech, D.; Popescu, I. C.; Gonaus, C.; Peterbauer, C. K.et al. Recombinant Pyranose Dehydrogenase-A Versatile Enzyme Possessing Both Mediated and Direct Electron Transfer. Electrochem. Commun. 2012, 24, 120-122.

(267) Bartlett, P. N.; Al-Lolage, F. A. There is No Evidence to Support Literature Claims of Direct Electron Transfer (DET) for Native Glucose Oxidase (GOx) at Carbon Nanotubes or Graphene. $J$. Electroanal. Chem. 2018, 819, 26-37.

(268) Wilson, G. S. Native Glucose Oxidase Does Not Undergo Direct Electron Transfer. Biosens. Bioelectron. 2016, 82, vii-viii.

(269) Wooten, M.; Karra, S.; Zhang, M.; Gorski, W. On the Direct Electron Transfer, Sensing, and Enzyme Activity in the Glucose Oxidase/Carbon Nanotubes System. Anal. Chem. 2014, 86, 752-757.

(270) Adachi, T.; Kitazumi, Y.; Shirai, O.; Kano, K. Construction of a Bioelectrochemical Formate Generating System from Carbon Dioxide and Dihydrogen. Electrochem. Commun. 2018, 97, 73-76.

(271) Heller, A. Electron-Conducting Redox Hydrogels: Design, Characteristics and Synthesis. Curr. Opin. Chem. Biol. 2006, 10, 664-672.

(272) Cosnier, S.; Holzinger, M. Electrosynthesized Polymers for Biosensing. Chem. Soc. Rev. 
2011, 40, 2146-2156.

(273) Cadet, M.; Gounel, S.; Stines-Chaumeil, C.; Brilland, X.; Rouhana, J.; Louerat, F.; Mano, N. An Enzymatic Glucose/ $\mathrm{O}_{2}$ Biofuel Cell Operating in Human Blood. Biosens. Bioelectron. 2016, 83, 60-67.

(274) van Rijt, S. H.; Sadler, P. J. Current Applications and Future Potential for Bioinorganic Chemistry in the Development of Anticancer Drugs. Drug Discov. Today 2009, 14, 1089-1097.

(275) Gross, A. J.; Chen, X.; Giroud, F.; Travelet, C.; Borsali, R.; Cosnier, S. Redox-Active Glyconanoparticles as Electron Shuttles for Mediated Electron Transfer with Bilirubin Oxidase in Solution. J. Am. Chem. Soc. 2017, 139, 16076-16079.

(276) Hammond, J. H.; Gross, A. J.; Giroud, F.; Travelet, C.; Redouane, B.; Cosnier, S. Solubilized Enzymatic Fuel Cell (SEFC) for Quasi-Continuous Operation Exploiting Carbohydrate Block Copolymer Glyconanoparticle Mediators. ACS Energy Lett. 2019, 4, 142-148.

(277) Tasca, F.; Gorton, L.; Harreither, W.; Haltrich, D.; Ludwig, R.; Nöll, G. Highly Efficient and Versatile Anodes for Biofuel Cells Based on Cellobiose Dehydrogenase from Myriococcum thermophilum. J. Phys. Chem. C 2008, 112, 13668-13673.

(278) Hodak, J.; Etchenique, R.; Calvo, E. J.; Singhal, K.; Bartlett, P. N. Layer-by-Layer Self-Assembly of Glucose Oxidase with a Poly(allylamine)ferrocene Redox Mediator. Langmuir 1997, 13, 2708-2716.

(279) Flexer, V.; Calvo, E. J.; Bartlett, P. N. The Application of the Relaxation and Simplex Method to the Analysis of Data for Glucose Electrodes Based on Glucose Oxidase Immobilised in an Osmium Redox Polymer. J. Electroanal. Chem. 2010, 646, 24-32.

(280) Godman, N. P.; DeLuca, J. L.; McCollum, S. R.; Schmidtke, D. W.; Glatzhofer, D. T. Electrochemical Characterization of Layer-By-Layer Assembled Ferrocene-Modified Linear Poly(ethylenimine)/Enzyme Bioanodes for Glucose Sensor and Biofuel Cell Applications. Langmuir 2016, 32, 3541-3551.

(281) Elouarzaki, K.; Cheng, D.; Fisher, A. C.; Lee, J.-M. Coupling Orientation and Mediation Strategies for Efficient Electron Transfer in Hybrid Biofuel Cells. Nat. Energy 2018, 3, 574-581.

(282) Siepenkoetter, T.; Salaj-Kosla, U.; Xiao, X.; Belochapkine, S.; Magner, E. Nanoporous Gold Electrodes with Tuneable Pore Sizes for Bioelectrochemical Applications. Electroanalysis 2016, 28, 2415-2423.

(283) Siepenkoetter, T.; Salaj-Kosla, U.; Xiao, X.; Conghaile, P. Ó.; Pita, M.; Ludwig, R.; Magner, E. Immobilization of Redox Enzymes on Nanoporous Gold Electrodes: Applications in Biofuel Cells. ChemPlusChem 2017, 82, 553-560.

(284) Scanlon, M. D.; Salaj-Kosla, U.; Belochapkine, S.; MacAodha, D.; Leech, D.; Ding, Y.; Magner, E. Characterization of Nanoporous Gold Electrodes for Bioelectrochemical Applications. Langmuir 2011, 28, 2251-2261.

(285) Ding, Y.; Kim, Y. J.; Erlebacher, J. Nanoporous Gold Leaf: “Ancient Technology”/Advanced Material. Adv. Mater. 2004, 16, 1897-1900.

(286) Hou, C.; Yang, D.; Liang, B.; Liu, A. Enhanced Performance of a Glucose/ $\mathrm{O}_{2}$ Biofuel Cell Assembled with Laccase-Covalently Immobilized Three-Dimensional Macroporous Gold Film-Based Biocathode and Bacterial Surface Displayed Glucose Dehydrogenase-Based Bioanode. Anal. Chem. 
2014, $86,6057-6063$.

(287) Murata, K.; Kajiya, K.; Nakamura, N.; Ohno, H. Direct Electrochemistry of Bilirubin Oxidase on Three-Dimensional Gold Nanoparticle Electrodes and Its Application in a Biofuel Cell. Energy Environ. Sci. 2009, 2, 1280-1285.

(288) Wang, X.; Falk, M.; Ortiz, R.; Matsumura, H.; Bobacka, J.; Ludwig, R.; Bergelin, M.; Gorton, L.; Shleev, S. Mediatorless Sugar/Oxygen Enzymatic Fuel Cells Based on Gold Nanoparticle-Modified Electrodes. Biosens. Bioelectron. 2012, 31, 219-225.

(289) Falk, M.; Alcalde, M.; Bartlett, P. N.; De Lacey, A. L.; Gorton, L.; Gutierrez-Sanchez, C.; Haddad, R.; Kilburn, J.; Leech, D.; Ludwig, R.et al. Self-Powered Wireless Carbohydrate/Oxygen Sensitive Biodevice Based on Radio Signal Transmission. PLOS ONE 2014, 9, e109104.

(290) Nishio, K.; Masuda, H. Anodization of Gold in Oxalate Solution to Form a Nanoporous Black Film. Angew. Chem. Int. Ed. 2011, 50, 1603-1607.

(291) R. Szamocki; S. Reculusa; S. Ravaine; P. N. Bartlett; A. Kuhn; R. Hempelmann. Tailored Mesostructuring and Biofunctionalization of Gold for Increased Electroactivity. Angew. Chem. Int. Ed. 2006, 45, 1317-1321.

(292) Karajić, A.; Reculusa, S.; Heim, M.; Garrigue, P.; Ravaine, S.; Mano, N.; Kuhn, A. Bottom-up Generation of Miniaturized Coaxial Double Electrodes with Tunable Porosity. Adv. Mater. Interfaces 2015, 2, 1500192.

(293) Boland, S.; Leech, D. A Glucose/Oxygen Enzymatic Fuel Cell Based on Redox Polymer and Enzyme Immobilisation at Highly-Ordered Macroporous Gold Electrodes. Analyst 2012, 137, 113-117. (294) du Toit, H.; Di Lorenzo, M. Continuous Power Generation from Glucose with Two Different Miniature Flow-Through Enzymatic Biofuel Cells. Biosens. Bioelectron. 2015, 69, 199-205.

(295) Xiao, X.; Li, H.; Zhang, K.; Si, P. Examining the Effects of Self-Assembled Monolayers on Nanoporous Gold Based Amperometric Glucose Biosensors. Analyst 2014, 139, 488-494.

(296) Pita, M.; Gutierrez-Sanchez, C.; Toscano, M. D.; Shleev, S.; De Lacey, A. L. Oxygen Biosensor Based on Bilirubin Oxidase Immobilized on a Nanostructured Gold Electrode. Bioelectrochemistry 2013, 94, 69-74.

(297) Siepenkoetter, T.; Salaj-Kosla, U.; Magner, E. The Immobilization of Fructose Dehydrogenase on Nanoporous Gold Electrodes for the Detection of Fructose. ChemElectroChem 2017, 4, 905-912.

(298) Gao, Z.; Binyamin, G.; Kim, H. H.; Barton, S. C.; Zhang, Y.; Heller, A. Electrodeposition of Redox Polymers and Co-Electrodeposition of Enzymes by Coordinative Crosslinking. Angew. Chem. Int. Ed. 2002, 41, 810-813.

(299) Xiao, X.; Wang, M. e.; Li, H.; Si, P. One-Step Fabrication of Bio-Functionalized Nanoporous Gold/Poly (3, 4-Ethylenedioxythiophene) Hybrid Electrodes for Amperometric Glucose Sensing. Talanta 2013, 115, 1054-1059.

(300) Salaj-Kosla, U.; Scanlon, M. D.; Baumeister, T.; Zahma, K.; Ludwig, R.; Conghaile, P. Ó.; MacAodha, D.; Leech, D.; Magner, E. Mediated Electron Transfer of Cellobiose Dehydrogenase and Glucose Oxidase at Osmium Polymer-Modified Nanoporous Gold Electrodes. Anal. Bioanal. Chem. 2013, 405, 3823-3830.

(301) Deng, L.; Wang, F.; Chen, H.; Shang, L.; Wang, L.; Wang, T.; Dong, S. A Biofuel Cell with 
Enhanced Performance by Multilayer Biocatalyst Immobilized on Highly Ordered Macroporous Electrode. Biosens. Bioelectron. 2008, 24, 329-333.

(302) Chen, L. Y.; Fujita, T.; Chen, M. W. Biofunctionalized Nanoporous Gold for Electrochemical Biosensors. Electrochim. Acta 2012, 67, 1-5.

(303) Hou, C.; Liu, A. An Integrated Device of Enzymatic Biofuel Cells and Supercapacitor for Both Efficient Electric Energy Conversion and Storage. Electrochim. Acta 2017, 245, 303-308.

(304) Yu, Y.; Han, Y.; Xu, M.; Zhang, L.; Dong, S. Automatic Illumination Compensation Device Based on a Photoelectrochemical Biofuel Cell Driven by Visible Light. Nanoscale 2016, 8, 9004-9008. (305) Reid, R. C.; Jones, S. R.; Hickey, D. P.; Minteer, S. D.; Gale, B. K. Modeling Carbon Nanotube Connectivity and Surface Activity in a Contact Lens Biofuel Cell. Electrochim. Acta 2016, 203, 30-40.

(306) Ó Conghaile, P.; Falk, M.; MacAodha, D.; Yakovleva, M. E.; Gonaus, C.; Peterbauer, C. K.; Gorton, L.; Shleev, S.; Leech, D. Fully Enzymatic Membraneless Glucose|Oxygen Fuel Cell That Provides $0.275 \mathrm{~mA} \mathrm{~cm}^{-2}$ in $5 \mathrm{mM}$ Glucose, Operates in Human Physiological Solutions, and Powers Transmission of Sensing Data. Anal. Chem. 2016, 88, 2156-2163.

(307) Giroud, F.; Sawada, K.; Taya, M.; Cosnier, S. 5,5-Dithiobis(2-Nitrobenzoic Acid) Pyrene Derivative-Carbon Nanotube Electrodes for NADH Electrooxidation and Oriented Immobilization of Multicopper Oxidases for the Development of Glucose/ $\mathrm{O}_{2}$ Biofuel Cells. Biosens. Bioelectron. 2017, 87, 957-963.

(308) Yoshino, S.; Miyake, T.; Yamada, T.; Hata, K.; Nishizawa, M. Molecularly Ordered Bioelectrocatalytic Composite Inside a Film of Aligned Carbon Nanotubes. Adv. Energy Mater. 2013, 3, 60-64.

(309) Muguruma, H.; Iwasa, H.; Hidaka, H.; Hiratsuka, A.; Uzawa, H. Mediatorless Direct Electron Transfer between Flavin Adenine Dinucleotide-Dependent Glucose Dehydrogenase and Single-Walled Carbon Nanotubes. ACS Catal. 2017, 7, 725-734.

(310) Holzinger, M.; Le Goff, A.; Cosnier, S. Carbon Nanotube/Enzyme Biofuel Cells. Electrochim. Acta 2012, 82, 179-190.

(311) Zhong, Z.; Qian, L.; Tan, Y.; Wang, G.; Yang, L.; Hou, C.; Liu, A. A High-Performance Glucose/Oxygen Biofuel Cell Based on Multi-Walled Carbon Nanotube Films with Electrophoretic Deposition. J. Electroanal. Chem. 2018, 823, 723-729.

(312) Xia, H. Q.; Kitazumi, Y.; Shirai, O.; Ozawa, H.; Onizuka, M.; Komukai, T.; Kano, K. Factors Affecting the Interaction Between Carbon Nanotubes and Redox Enzymes in Direct Electron Transfer-Type Bioelectrocatalysis. Bioelectrochem. 2017, 118, 70-74.

(313) Bai, L.; Jin, L.; Han, L.; Dong, S. Self-Powered Fluorescence Controlled Wwitch Systems Based on Biofuel Cells. Energy Environ. Sci. 2013, 6, 3015-3021.

(314) Gumeci, C.; Do, D.; Barton, S. C. Electrospun Carbon Nanofibers as Supports for Bioelectrodes. Electrocatalysis 2017, 8, 321-328.

(315) Komori, K.; Huang, J.; Mizushima, N.; Ko, S.; Tatsuma, T.; Sakai, Y. Controlled Direct Electron Transfer Kinetics of Fructose Dehydrogenase at Cup-Stacked Carbon Nanofibers. Phys. Chem. Chem. Phys. 2017, 19, 27795-27800.

(316) Senthamizhan, A.; Balusamy, B.; Uyar, T. Glucose Sensors Based on Electrospun Nanofibers: A Review. Anal. Bioanal. Chem. 2016, 408, 1285-1306. 
Bourourou, M.; Holzinger, M.; Elouarzaki, K.; Le Goff, A.; Bossard, F.; Rossignol, C.; Djurado, E.; Martin, V.; Curtil, D.; Chaussy, D.et al. Laccase Wiring on Free-Standing Electrospun Carbon Nanofibres Using a Mediator Plug. Chem. Commun. 2015, 51, 14574-14577.

(318) Sim, H. J.; Lee, D. Y.; Kim, H.; Choi, Y.-B.; Kim, H.-H.; Baughman, R. H.; Kim, S. J. Stretchable Fiber Biofuel Cell by Rewrapping Multiwalled Carbon Nanotube Sheets. Nano Lett. 2018, $18,5272-5278$.

(319) Filip, J.; Tkac, J. Is Graphene Worth Using in Biofuel Cells? Electrochim. Acta 2014, 136, 340-354.

(320) Tsujimura, S.; Murata, K.; Akatsuka, W. Exceptionally High Glucose Current on a Hierarchically Structured Porous Carbon Electrode with "Wired" Flavin Adenine Dinucleotide-Dependent Glucose Dehydrogenase. J. Am. Chem. Soc. 2014, 136, 14432-14437.

(321) Funabashi, H.; Takeuchi, S.; Tsujimura, S. Hierarchical Meso/Macro-Porous Carbon Fabricated from Dual $\mathrm{MgO}$ Templates for Direct Electron Transfer Enzymatic Electrodes. Sci. Rep. 2017, 7, 45147.

(322) Wu, G.; Gao, Y.; Zhao, D.; Ling, P.; Gao, F. Methanol/Oxygen Enzymatic Biofuel Cell Using Laccase and NAD+-Dependent Dehydrogenase Cascades as Biocatalysts on Carbon Nanodots Electrodes. ACS Appl. Mater. Interfaces 2017, 9, 40978-40986.

(323) MacVittie, K.; Conlon, T.; Katz, E. A Wireless Transmission System Powered by an Enzyme Biofuel Cell Implanted in an Orange. Bioelectrochemistry 2015, 106, 28-33.

(324) Slaughter, G.; Kulkarni, T. A Self-Powered Glucose Biosensing System. Biosens. Bioelectron. 2016, 78, 45-50.

(325) Hickey, D. P.; Milton, R. D.; Rasmussen, M.; Abdellaoui, S.; Nguyen, K.; Minteer, S. D. In Electrochemistry; The Royal Society of Chemistry, 2016; Vol. 13.

(326) Koushanpour, A.; Guz, N.; Gamella, M.; Katz, E. Biofuel Cell Based on Carbon Fiber Electrodes Functionalized with Graphene Nanosheets. ECS J. Solid State Sci. Technol. 2016, 5, M3037-M3040.

(327) Ouyang, J.; Liu, Z.; Han, Y.; Zeng, K.; Sheng, J.; Deng, L.; Liu, Y.-N. Fabrication of Surface Protein-Imprinted Biofuel Cell for Sensitive Self-Powered Glycoprotein Detection. ACS Appl. Mater. Interfaces 2016, 8, 35004-35011.

(328) Poulpiquet, A. d.; Marques-Knopf, H.; Wernert, V.; Giudici-Orticoni, M. T.; Gadiou, R.; Lojou, E. Carbon Nanofiber Mesoporous Films: Efficient Platforms for Bio-Hydrogen Oxidation in Biofuel Cells. Phys. Chem. Chem. Phys. 2014, 16, 1366-1378.

(329) Song, Y.; Chen, C.; Wang, C. Graphene/Enzyme-Encrusted Three-Dimensional Carbon Micropillar Arrays for Mediatorless Micro-Biofuel Cells. Nanoscale 2015, 7, 7084-7090.

(330) Lalaoui, N.; Holzinger, M.; Le Goff, A.; Cosnier, S. Diazonium Functionalisation of Carbon Nanotubes for Specific Orientation of Multicopper Oxidases: Controlling Electron Entry Points and Oxygen Diffusion to the Enzyme. Chem. Eur. J. 2016, 22, 10494-10500.

(331) Sosna, M.; Stoica, L.; Wright, E.; Kilburn, J. D.; Schuhmann, W.; Bartlett, P. N. Mass Transport Controlled Oxygen Reduction at Anthraquinone Modified 3D-CNT Electrodes with Immobilized Trametes hirsuta Laccase. Phys. Chem. Chem. Phys. 2012, 14, 11882-11885.

(332) Ghanem, M. A.; Kocak, I.; Al-Mayouf, A.; AlHoshan, M.; Bartlett, P. N. Covalent Modification of Carbon Nanotubes with Anthraquinone by Electrochemical Grafting and Solid Phase 
Synthesis. Electrochim. Acta 2012, 68, 74-80.

(333) Elouarzaki, K.; Bourourou, M.; Holzinger, M.; Le Goff, A.; Marks, R. S.; Cosnier, S. Freestanding HRP-GOx Redox Buckypaper as an Oxygen-Reducing Biocathode for Biofuel Cell Applications. Energy Environ. Sci. 2015, 8, 2069-2074.

(334) Habermüller, K.; Ramanavicius, A.; Laurinavicius, V.; Schuhmann, W. An Oxygen-Insensitive Reagentless Glucose Biosensor Based on Osmium-Complex Modified Polypyrrole. Electroanalysis 2000, 12, 1383-1389.

(335) Monsalve, K.; Mazurenko, I.; Gutierrez-Sanchez, C.; Ilbert, M.; Infossi, P.; Frielingsdorf, S.; Giudici-Orticoni, M. T.; Lenz, O.; Lojou, E. Impact of Carbon Nanotube Surface Chemistry on Hydrogen Oxidation by Membrane-Bound Oxygen-Tolerant Hydrogenases. ChemElectroChem 2016, 3 , 2179-2188.

(336) Trohalaki, S.; Pachter, R.; Luckarift, H. R.; Johnson, G. R. Immobilization of the Laccases from Trametes versicolor and Streptomyces coelicolor on Single-Wall Carbon Nanotube Electrodes: A Molecular Dynamics Study. Fuel Cells 2012, 12, 656-664.

(337) Singh, M.; Holzinger, M.; Biloivan, O.; Cosnier, S. 3D-Nanostructured Scaffold Electrodes Based on Single-Walled Carbon Nanotubes and Nanodiamonds for High Performance Biosensors. Carbon 2013, 61, 349-356.

(338) Ciaccafava, A.; De Poulpiquet, A.; Techer, V.; Giudici-Orticoni, M. T.; Tingry, S.; Innocent, C.; Lojou, E. An Innovative Powerful and Mediatorless $\mathrm{H}_{2 /} \mathrm{O}_{2}$ Biofuel Cell Based on an Outstanding Bioanode. Electrochem. Commun. 2012, 23, 25-28.

(339) Chen, T.; Barton, S. C.; Binyamin, G.; Gao, Z.; Zhang, Y.; Kim, H.-H.; Heller, A. A Miniature Biofuel Cell. J. Am. Chem. Soc. 2001, 123, 8630-8631.

(340) Mano, N.; Mao, F.; Heller, A. Characteristics of a Miniature Compartment-less Glucose- $-\mathrm{O}_{2}$ Biofuel Cell and Its Operation in a Living Plant. J. Am. Chem. Soc. 2003, 125, 6588-6594.

(341) Miyake, T.; Haneda, K.; Nagai, N.; Yatagawa, Y.; Onami, H.; Yoshino, S.; Abe, T.; Nishizawa, M. Enzymatic Biofuel Cells Designed for Direct Power Generation from Biofluids in Living Organisms. Energy Environ. Sci. 2011, 4, 5008-5012.

(342) Sales, F. C. P. F.; Iost, R. M.; Martins, M. V. A.; Almeida, M. C.; Crespilho, F. N. An Intravenous Implantable Glucose/Dioxygen Biofuel Cell with Modified Flexible Carbon Fiber Electrodes. Lab Chip 2013, 13, 468-474.

(343) Orecchioni, M.; Ménard-Moyon, C.; Delogu, L. G.; Bianco, A. Graphene and the Immune System: Challenges and Potentiality. Adv. Drug Deliv. Rev. 2016, 105, 163-175.

(344) Magrez, A.; Kasas, S.; Salicio, V.; Pasquier, N.; Seo, J. W.; Celio, M.; Catsicas, S.; Schwaller, B.; Forró, L. Cellular Toxicity of Carbon-Based Nanomaterials. Nano Lett. 2006, 6, 1121-1125.

(345) Du, J.; Wang, S.; You, H.; Zhao, X. Understanding the Toxicity of Carbon Nanotubes in the Environment is Crucial to the Control of Nanomaterials in Producing and Processing and the Assessment of Health Risk for Human: A Review. Environ. Toxicol. Pharmacol. 2013, 36, 451-462.

(346) Flexer, V.; Brun, N.; Backov, R.; Mano, N. Designing Highly Efficient Enzyme-Based Carbonaceous Foams Electrodes for Biofuel Cells. Energy Environ. Sci. 2010, 3, 1302-1306.

(347) Mazurenko, I.; Clément, R.; Byrne-Kodjabachian, D.; de Poulpiquet, A.; Tsujimura, S.; Lojou, E. Pore Size Effect of MgO-Templated Carbon on Enzymatic $\mathrm{H}_{2}$ Oxidation by the Hyperthermophilic Hydrogenase from Aquifex aeolicus. J. Electroanal. Chem. 2018, 812, 221-226. 
(348) Kontani, R.; Tsujimura, S.; Kano, K. Air Diffusion Biocathode with CueO as Electrocatalyst Adsorbed on Carbon Particle-Modified Electrodes. Bioelectrochemistry 2009, 76, 10-13.

(349) Lalaoui, N.; de Poulpiquet, A.; Haddad, R.; Le Goff, A.; Holzinger, M.; Gounel, S.; Mermoux, M.; Infossi, P.; Mano, N.; Lojou, E.et al. A membraneless air-breathing hydrogen biofuel cell based on direct wiring of thermostable enzymes on carbon nanotube electrodes. Chem. Commun. 2015, 51, 7447-7450.

(350) Shleev, S.; Shumakovich, G.; Morozova, O.; Yaropolov, A. Stable 'Floating' Air Diffusion Biocathode Based on Direct Electron Transfer Reactions Between Carbon Particles and High Redox Potential Laccase. Fuel Cells 2010, 10, 726-733.

(351) Nakagawa, T.; Mita, H.; Kumita, H.; Sakai, H.; Tokita, Y.; Tsujimura, S. Water-Repellent-Treated Enzymatic Electrode for Passive Air-Breathing Biocathodic Reduction of Oxygen. Electrochem. Commun. 2013, 36, 46-49.

(352) So, K.; Onizuka, M.; Komukai, T.; Kitazumi, Y.; Shirai, O.; Kano, K. Binder/Surfactant-Free Biocathode with Bilirubin Oxidase for Gas-Diffusion-Type System. Electrochem. Commun. 2016, 66, 58-61.

(353) Tarasevich, M. R.; Bogdanovskaya, V. A.; Kapustin, A. V. Nanocomposite Material Laccase/Dispersed Carbon Carrier for Oxygen Electrode. Electrochem. Commun. 2003, 5, 491-496.

(354) Gupta, G.; Lau, C.; Rajendran, V.; Colon, F.; Branch, B.; Ivnitski, D.; Atanassov, P. Direct Electron Transfer Catalyzed by Bilirubin Oxidase for Air Breathing Gas-Diffusion Electrodes. Electrochem. Commun. 2011, 13, 247-249.

(355) Gupta, G.; Lau, C.; Branch, B.; Rajendran, V.; Ivnitski, D.; Atanassov, P. Direct Bio-Electrocatalysis by Multi-Copper Oxidases: Gas-Diffusion Laccase-Catalyzed Cathodes for Biofuel Cells. Electrochim. Acta 2011, 56, 10767-10771.

(356) Rincón, R. A.; Lau, C.; Luckarift, H. R.; Garcia, K. E.; Adkins, E.; Johnson, G. R.; Atanassov, P. Enzymatic Fuel Cells: Integrating Flow-Through Anode and Air-Breathing Cathode into a Membrane-Less Biofuel Cell Design. Biosens. Bioelectron. 2011, 27, 132-136.

(357) Higgins, S. R.; Lau, C.; Atanassov, P.; Minteer, S. D.; Cooney, M. J. Hybrid Biofuel Cell: Microbial Fuel Cell with an Enzymatic Air-Breathing Cathode. ACS Catal. 2011, 1, 994-997.

(358) Lau, C.; Adkins, E. R.; Ramasamy, R. P.; Luckarift, H. R.; Johnson, G. R.; Atanassov, P. Design of Carbon Nanotube-Based Gas-Diffusion Cathode for $\mathrm{O}_{2}$ Reduction by Multicopper Oxidases. Adv. Energy Mater. 2012, 2, 162-168.

(359) So, K.; Kitazumi, Y.; Shirai, O.; Kurita, K.; Nishihara, H.; Higuchi, Y.; Kano, K. Gas-Diffusion and Direct-Electron-Transfer-Type Bioanode for Hydrogen Oxidation with Oxygen-Tolerant [NiFe]-Hydrogenase as an Electrocatalyst. Chem. Lett. 2014, 43, 1575-1577.

(360) Sakai, K.; Kitazumi, Y.; Shirai, O.; Takagi, K.; Kano, K. Efficient Bioelectrocatalytic $\mathrm{CO}_{2}$ Reduction on Gas-Diffusion-Type Biocathode with Tungsten-Containing Formate Dehydrogenase. Electrochem. Commun. 2016, 73, 85-88.

(361) Ciniciato, G. P. M. K.; Lau, C.; Cochrane, A.; Sibbett, S. S.; Gonzalez, E. R.; Atanassov, P. Development of Paper Based Electrodes: From Air-Breathing to Paintable Enzymatic Cathodes. Electrochim. Acta 2012, 82, 208-213.

(362) Narváez Villarrubia, C. W.; Lau, C.; Ciniciato, G. P. M. K.; Garcia, S. O.; Sibbett, S. S.; Petsev, D. N.; Babanova, S.; Gupta, G.; Atanassov, P. Practical Electricity Generation from a Paper 
Based Biofuel Cell Powered by Glucose in Ubiquitous Liquids. Electrochem. Commun. 2014, 45, 44-47.

(363) Lau, C.; Moehlenbrock, M. J.; Arechederra, R. L.; Falase, A.; Garcia, K.; Rincon, R.; Minteer, S. D.; Banta, S.; Gupta, G.; Babanova, S.et al. Paper Based Biofuel Cells: Incorporating Enzymatic Cascades for Ethanol and Methanol Oxidation. Int. J. Hydrogen Energy 2015, 40, 14661-14666.

(364) Narvaez Villarrubia, C. W.; Soavi, F.; Santoro, C.; Arbizzani, C.; Serov, A.; Rojas-Carbonell, S.; Gupta, G.; Atanassov, P. Self-Feeding Paper Based Biofuel Cell/Self-Powered Hybrid $\mu$-Supercapacitor Integrated System. Biosens. Bioelectron. 2016, 86, 459-465.

(365) Mi, L.; Yu, J.; He, F.; Jiang, L.; Wu, Y.; Yang, L.; Han, X.; Li, Y.; Liu, A.; Wei, W.et al. Boosting Gas Involved Reactions at Nanochannel Reactor with Joint Gas-Solid-Liquid Interfaces and Controlled Wettability. J. Am. Chem. Soc. 2017, 139, 10441-10446.

(366) Moore, C. M.; Minteer, S. D.; Martin, R. S. Microchip-Based Ethanol/Oxygen Biofuel Cell. Lab Chip 2005, 5, 218-225.

(367) Lim, K. G.; Palmore, G. T. R. Microfluidic Biofuel Cells: The Influence of Electrode Diffusion Layer on Performance. Biosens. Bioelectron. 2007, 22, 941-947.

(368) Zebda, A.; Renaud, L.; Cretin, M.; Innocent, C.; Pichot, F.; Ferrigno, R.; Tingry, S. Electrochemical Performance of a Glucose/Oxygen Microfluidic Biofuel Cell. J. Power Sources 2009, 193, 602-606.

(369) Zebda, A.; Renaud, L.; Cretin, M.; Innocent, C.; Ferrigno, R.; Tingry, S. Membraneless microchannel glucose biofuel cell with improved electrical performances. Sens. Actuat. B: Chem. 2010, 149, 44-50.

(370) Togo, M.; Takamura, A.; Asai, T.; Kaji, H.; Nishizawa, M. An Enzyme-Based Microfluidic Biofuel Cell Using Vitamin $\mathrm{K}_{3}$-Mediated Glucose Oxidation. Electrochim. Acta 2007, 52, 4669-4674.

(371) Beneyton, T.; Wijaya, I. P. M.; Salem, C. B.; Griffiths, A. D.; Taly, V. Membraneless Glucose/ $\mathrm{O}_{2}$ Microfluidic Biofuel Cells Using Covalently Bound Enzymes. Chem. Commun. 2013, 49, 1094-1096.

(372) Abreu, C.; Nedellec, Y.; Ondel, O.; Buret, F.; Cosnier, S.; Le Goff, A.; Holzinger, M. Glucose Oxidase Bioanodes for Glucose Conversion and $\mathrm{H}_{2} \mathrm{O}_{2}$ Production for Horseradish Peroxidase Biocathodes in a Flow Through Glucose Biofuel Cell Design. J. Power Sources 2018, 392, 176-180.

(373) Abreu, C.; Nedellec, Y.; Gross, A. J.; Ondel, O.; Buret, F.; Goff, A. L.; Holzinger, M.; Cosnier, S. Assembly and Stacking of Flow-Through Enzymatic Bioelectrodes for High Power Glucose Fuel Cells. ACS Appl. Mater. Interfaces 2017, 9, 23836-23842.

(374) Gonzalez-Guerrero, M. J.; Esquivel, J. P.; Sanchez-Molas, D.; Godignon, P.; Munoz, F. X.; del Campo, F. J.; Giroud, F.; Minteer, S. D.; Sabate, N. Membraneless Glucose/O $\mathrm{O}_{2}$ Microfluidic Enzymatic Biofuel Cell Using Pyrolyzed Photoresist Film Electrodes. Lab Chip 2013, 13, 2972-2979.

(375) Dector, A.; Escalona-Villalpando, R. A.; Dector, D.; Vallejo-Becerra, V.; Chávez-Ramírez, A. U.; Arriaga, L. G.; Ledesma-García, J. Perspective Use of Direct Human Blood as an Energy Source in Air-Breathing Hybrid Microfluidic Fuel Cells. J. Power Sources 2015, 288, 70-75.

(376) Escalona-Villalpando, R. A.; Reid, R. C.; Milton, R. D.; Arriaga, L. G.; Minteer, S. D.; Ledesma-García, J. Improving the Performance of Lactate/Oxygen Biofuel Cells Using a Microfluidic Design. J. Power Sources 2017, 342, 546-552. 
Porous Electrodes into Microfluidic Co-Laminar Enzymatic Biofuel Cells: Toward Higher Enzyme Loadings for Enhanced Performance. Microelectron. Eng. 2016, 165, 23-26.

(378) Pankratov, D.; Ohlsson, L.; Gudmundsson, P.; Halak, S.; Ljunggren, L.; Blum, Z.; Shleev, S. Ex vivo Electric Power Generation in Human Blood Using an Enzymatic Fuel Cell in a Vein Replica. RSC Adv. 2016, 6, 70215-70220.

(379) Winter, M.; Brodd, R. J. What Are Batteries, Fuel Cells, and Supercapacitors? Chem. Rev. 2004, 104, 4245-4270.

(380) Skunik-Nuckowska, M.; Grzejszczyk, K.; Stolarczyk, K.; Bilewicz, R.; Kulesza, P. J. Integration of Supercapacitors with Enzymatic Biobatteries toward More Effective Pulse-Powered Use in Small-Scale Energy Harvesting Devices. J. Appl. Electrochem. 2014, 44, 497-507.

(381) Hanashi, T.; Yamazaki, T.; Tsugawa, W.; Ferri, S.; Nakayama, D.; Tomiyama, M.; Ikebukuro, K.; Sode, K. BioCapacitor-A Novel Category of Biosensor. Biosens. Bioelectron. 2009, 24, 1837-1842.

(382) Monsalve, K.; Mazurenko, I.; Lalaoui, N.; Le Goff, A.; Holzinger, M.; Infossi, P.; Nitsche, S.;

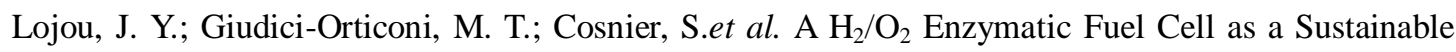
Power for a Wireless Device. Electrochem. Commun. 2015, 60, 216-220.

(383) Agnes, C.; Holzinger, M.; Le Goff, A.; Reuillard, B.; Elouarzaki, K.; Tingry, S.; Cosnier, S. Supercapacitor/Biofuel Cell Hybrids Based on Wired Enzymes on Carbon Nanotube Matrices: Autonomous Reloading after High Power Pulses in Neutral Buffered Glucose Solutions. Energy Environ. Sci. 2014, 7, 1884-1888.

(384) Pankratov, D.; Blum, Z.; Suyatin, D. B.; Popov, V. O.; Shleev, S. Self-Charging Electrochemical Biocapacitor. ChemElectroChem 2014, 1, 343-346.

(385) Pankratov, D.; Blum, Z.; Shleev, S. Hybrid Electric Power Biodevices. ChemElectroChem 2014, 1, 1798-1807.

(386) Santoro, C.; Soavi, F.; Serov, A.; Arbizzani, C.; Atanassov, P. Self-Powered Supercapacitive Microbial Fuel Cell: The Ultimate Way of Boosting and Harvesting Power. Biosens. Bioelectron. 2016, $78,229-235$.

(387) Kizling, M.; Stolarczyk, K.; Kiat, J. S. S.; Tammela, P.; Wang, Z.; Nyholm, L.; Bilewicz, R. Pseudocapacitive Polypyrrole-Nanocellulose Composite for Sugar-Air Enzymatic Fuel Cells. Electrochem. Commun. 2015, 50, 55-59.

(388) Kizling, M.; Draminska, S.; Stolarczyk, K.; Tammela, P.; Wang, Z.; Nyholm, L.; Bilewicz, R. Biosupercapacitors for Powering Oxygen Sensing Devices. Bioelectrochemistry 2015, 106, Part A, 34-40.

(389) Knoche, K. L.; Hickey, D. P.; Milton, R. D.; Curchoe, C. L.; Minteer, S. D. Hybrid Glucose/ $\mathrm{O}_{2}$ Biobattery and Supercapacitor Utilizing a Pseudocapacitive Dimethylferrocene Redox Polymer at the Bioanode. ACS Energy Lett. 2016, 1, 380-385.

(390) Alsaoub, S.; Ruff, A.; Conzuelo, F.; Ventosa, E.; Ludwig, R.; Shleev, S.; Schuhmann, W. An Intrinsic Self-Charging Biosupercapacitor Comprised of a High-Potential Bioanode and a Low-Potential Biocathode. ChemPlusChem 2017, 82, 576-583.

(391) Mosa, I. M.; Pattammattel, A.; Kadimisetty, K.; Pande, P.; El-Kady, M. F.; Bishop, G. W.; Novak, M.; Kaner, R. B.; Basu, A. K.; Kumar, C. V.et al. Ultrathin Graphene-Protein Supercapacitors 
for Miniaturized Bioelectronics. Adv. Energy Mater. 2017, 7, 1700358.

(392) Xiao, X.; Conghaile, P. Ó.; Leech, D.; Ludwig, R.; Magner, E. A Symmetric Supercapacitor/Biofuel Cell Hybrid Device Based on Enzyme-Modified Nanoporous Gold: An Autonomous Pulse Generator. Biosens. Bioelectron. 2017, 90, 96-102.

(393) Shen, F.; Pankratov, D.; Pankratova, G.; Toscano, M. D.; Zhang, J.; Ulstrup, J.; Chi, Q.; Gorton, L. Supercapacitor/Biofuel Cell Hybrid Device Employing Biomolecules for Energy Conversion and Charge Storage. Bioelectrochem. 2019, 128, 94-99.

(394) Ahmad, R.; Sardar, M. Enzyme Immobilization: An Overview on Nanoparticles as Immobilization Matrix. Biochem. Anal. Biochem. 2015, 4, 1000178.

(395) Mateo, C.; Palomo, J. M.; Fernandez-Lorente, G.; Guisan, J. M.; Fernandez-Lafuente, R. Improvement of Enzyme Activity, Stability and Selectivity via Immobilization Techniques. Enzyme Microb. Technol. 2007, 40, 1451-1463.

(396) Lojou, E. Hydrogenases as Catalysts for Fuel Cells: Strategies for Efficient Immobilization at Electrode Interfaces. Electrochim. Acta 2011, 56, 10385-10397.

(397) Climent, V.; Zhang, J.; Friis, E. P.; Østergaard, L. H.; Ulstrup, J. Voltammetry and Single-Molecule in Situ Scanning Tunneling Microscopy of Laccases and Bilirubin Oxidase in Electrocatalytic Dioxygen Reduction on Au(111) Single-Crystal Electrodes. J. Phys. Chem. C 2011, $116,1232-1243$.

(398) Sugimoto, Y.; Kitazumi, Y.; Tsujimura, S.; Shirai, O.; Yamamoto, M.; Kano, K. Electrostatic Interaction between an Enzyme and Electrodes in the Electric Double Layer Examined in a View of Direct Electron Transfer-Type Bioelectrocatalysis. Biosens. Bioelectron. 2015, 63, 138-144.

(399) Salaj-Kosla, U.; Poller, S.; Schuhmann, W.; Shleev, S.; Magner, E. Direct Electron Transfer of Trametes hirsuta Laccase Adsorbed at Unmodified Nanoporous Gold Electrodes. Bioelectrochemistry 2013, 91, 15-20.

(400) Krikstolaityte, V.; Barrantes, A.; Ramanavicius, A.; Arnebrant, T.; Shleev, S.; Ruzgas, T. Bioelectrocatalytic Reduction of Oxygen at Gold Nanoparticles Modified with Laccase. Bioelectrochemistry 2014, 95, 1-6.

(401) Dagys, M.; Haberska, K.; Shleev, S.; Arnebrant, T.; Kulys, J.; Ruzgas, T. Laccase-Gold Nanoparticle Assisted Bioelectrocatalytic Reduction of Oxygen. Electrochem. Commun. 2010, 12, 933-935.

(402) Qiu, H.; Xu, C.; Huang, X.; Ding, Y.; Qu, Y.; Gao, P. Adsorption of Laccase on the Surface of Nanoporous Gold and Direct Electron Transfer between Them. J. Phys. Chem. C 2008, 112, 14781-14785.

(403) Bellino, M. G.; Soler-Illia, G. J. A. A. Nano-Designed Enzyme-Functionalized Hierarchical Metal-Oxide Mesoporous Thin Films: En Route to Versatile Biofuel Cells. Small 2014, 10, 2834-2839. (404) Funabashi, H.; Murata, K.; Tsujimura, S. Effect of Pore Size of MgO-Templated Carbon on the Direct Electrochemistry of $D$-Fructose Dehydrogenase. Electrochemistry 2015, 83, 372-375.

(405) Takahashi, H.; Li, B.; Sasaki, T.; Miyazaki, C.; Kajino, T.; Inagaki, S. Catalytic Activity in Organic Solvents and Stability of Immobilized Enzymes Depend on the Pore Size and Surface Characteristics of Mesoporous Silica. Chem. Mater. 2000, 12, 3301-3305.

(406) Lamberg, P.; Hamit-Eminovski, J.; Toscano, M. D.; Eicher-Lorka, O.; Niaura, G.; Arnebrant, T.; Shleev, S.; Ruzgas, T. Electrical Activity of Cellobiose Dehydrogenase Adsorbed on Thiols: 
Influence of Charge and Hydrophobicity. Bioelectrochemistry 2017, 115, 26-32.

(407) Bourourou, M.; Elouarzaki, K.; Lalaoui, N.; Agnes, C.; Le Goff, A.; Holzinger, M.; Maaref, A.; Cosnier, S. Supramolecular Immobilization of Laccase on Carbon Nanotube Electrodes Functionalized with (Methylpyrenylaminomethyl)Anthraquinone for Direct Electron Reduction of Oxygen. Chemistry 2013, 19, 9371-9375.

(408) Xia, H.-q.; Kitazumi, Y.; Shirai, O.; Kano, K. Enhanced Direct Electron Transfer-Type Bioelectrocatalysis of Bilirubin Oxidase on Negatively Charged Aromatic Compound-Modified Carbon Electrode. J. Electroanal. Chem. 2016, 763, 104-109.

(409) Matanovic, I.; Babanova, S.; Chavez, M. S.; Atanassov, P. Protein-Support Interactions for Rationally Designed Bilirubin Oxidase Based Cathode: A Computational Study. J. Phys. Chem. B 2016, 120,3634-3641.

(410) Tasca, F.; Harreither, W.; Ludwig, R.; Gooding, J. J.; Gorton, L. Cellobiose Dehydrogenase Aryl Diazonium Modified Single Walled Carbon Nanotubes: Enhanced Direct Electron Transfer through a Positively Charged Surface. Anal. Chem. 2011, 83, 3042-3049.

(411) Minson, M.; Meredith, M. T.; Shrier, A.; Giroud, F.; Hickey, D.; Glatzhofer, D. T.; Minteer, S. D. High Performance Glucose/ $\mathrm{O}_{2}$ Biofuel Cell: Effect of Utilizing Purified Laccase with Anthracene-Modified Multi-Walled Carbon Nanotubes. J. Electrochem. Soc. 2012, 159, G166-G170.

(412) Pellissier, M.; Barrière, F.; Downard, A. J.; Leech, D. Improved Stability of Redox Enzyme Layers on Glassy Carbon Electrodes via Covalent Grafting. Electrochem. Commun. 2008, 10, 835-838.

(413) Shim, J.; Kim, G.-Y.; Moon, S.-H. Covalent Co-Immobilization of Glucose Oxidase and Ferrocenedicarboxylic Acid for an Enzymatic Biofuel Cell. J. Electroanal. Chem. 2011, 653, 14-20.

(414) Di Bari, C.; Shleev, S.; De Lacey, A. L.; Pita, M. Laccase-Modified Gold Nanorods for Eelectrocatalytic Reduction of Oxygen. Bioelectrochemistry 2016, 107, 30-36.

(415) Al-Lolage, F. A.; Bartlett, P. N.; Gounel, S.; Staigre, P.; Mano, N. Site-Directed Immobilization of Bilirubin Oxidase for Electrocatalytic Oxygen Reduction. ACS Catal. 2019, 9, 2068-2078.

(416) Soozanipour, A.; Taheri-Kafrani, A.; Landarani Isfahani, A. Covalent Attachment of Xylanase on Functionalized Magnetic Nanoparticles and Determination of Its Activity and Stability. Chem. Eng. J. 2015, 270, 235-243.

(417) Balistreri, N.; Gaboriau, D.; Jolivalt, C.; Launay, F. Covalent Immobilization of Glucose Oxidase on Mesocellular Silica Foams: Characterization and Stability Towards Temperature and Organic Solvents. J. Mol. Catal. B: Enzym. 2016, 127, 26-33.

(418) Ghanem, M. A.; Chrétien, J.-M.; Pinczewska, A.; Kilburn, J. D.; Bartlett, P. N. Covalent Modification of Glassy Carbon Surface with Organic Redox Probes through Diamine Linkers Using Electrochemical and Solid-Phase Synthesis Methodologies. J. Mater. Chem. 2008, 18, 4917-4927.

(419) Sosna, M.; Boer, H.; Bartlett, P. N. A His-Tagged Melanocarpus albomyces Laccase and its Electrochemistry upon Immobilisation on NTA-Modified Electrodes and in Conducting Polymer Films. ChemPhysChem 2013, 14, 2225-2231.

(420) Choi, O.; Kim, B. C.; An, J. H.; Min, K.; Kim, Y. H.; Um, Y.; Oh, M. K.; Sang, B. I. A Biosensor Based on the Self-Entrapment of Glucose Oxidase within Biomimetic Silica Nanoparticles Induced by a Fusion Enzyme. Enzyme Microb. Technol. 2011, 49, 441-445.

(421) Naik, R. R.; Tomczak, M. M.; Luckarift, H. R.; Spain, J. C.; Stone, M. O. Entrapment of 
Enzymes and Nanoparticles Using Biomimetically Synthesized Silica. Chem. Commun. 2004, 0, 1684-1685.

(422) Liu, C.; Alwarappan, S.; Chen, Z.; Kong, X.; Li, C. Z. Membraneless Enzymatic Biofuel Cells Based on Graphene Nanosheets. Biosens. Bioelectron. 2010, 25, 1829-1833.

(423) Ivnitski, D.; Artyushkova, K.; Rincon, R. A.; Atanassov, P.; Luckarift, H. R.; Johnson, G. R. Entrapment of Enzymes and Carbon Nanotubes in Biologically Synthesized Silica: Glucose Oxidase-catalyzed Direct Electron Transfer. Small 2008, 4, 357-364.

(424) Franco, A.; Cebrian-Garcia, S.; Rodriguez-Padron, D.; Puente-Santiago, A. R.; Munoz-Batista, M. J.; Caballero, A.; Balu, A.; Romero, A.; Luque, R. Encapsulated Laccases As Effective Electrocatalysts for Oxygen Reduction Reactions. ACS Sustainable Chem. Eng. 2018, 6, 11058.

(425) Grippo, V.; Pawłowska, J.; Biernat, J. F.; Bilewicz, R. Synergic Effect of Naphthylated Carbon Nanotubes and Gold Nanoparticles on Catalytic Performance of Hybrid Films Containing Bilirubin Oxidase for the Dioxygen Reduction. Electroanalysis 2017, 29, 103-109.

(426) Wu, G.; Yao, Z.; Fei, B.; Gao, F. An Enzymatic Ethanol Biosensor and Ethanol/Air Biofuel Cell Using Liquid-Crystalline Cubic Phases as Hosting Matrices to Co-Entrap Enzymes and Mediators. J. Electrochem. Soc. 2017, 164, G82-G86.

(427) Wang, S.-F.; Chen, T.; Zhang, Z.-L.; Pang, D.-W.; Wong, K.-Y. Effects of Hydrophilic Room-Temperature Ionic Liquid 1-Butyl-3-Methylimidazolium Tetrafluoroborate on Direct Electrochemistry and Bioelectrocatalysis of Heme Proteins Entrapped in Agarose Hydrogel Films. Electrochem. Commun. 2007, 9, 1709-1714.

(428) Nguyen, K. V.; Minteer, S. D. Investigating DNA Hydrogels as a New Biomaterial for Enzyme Immobilization in Biobatteries. Chem. Commun. 2015, 51, 13071-13073.

(429) El Ichi, S.; Zebda, A.; Laaroussi, A.; Reverdy-Bruas, N.; Chaussy, D.; Naceur Belgacem, M.; Cinquin, P.; Martin, D. K. Chitosan Improves Stability of Carbon Nanotube Biocathodes for Glucose Biofuel Cells. Chem. Commun. 2014, 50, 14535-14538.

(430) Reuillard, B.; Abreu, C.; Lalaoui, N.; Le Goff, A.; Holzinger, M.; Ondel, O.; Buret, F.; Cosnier, S. One-Year Stability for a Glucose/Oxygen Biofuel Cell Combined with $\mathrm{pH}$ Reactivation of the Laccase/Carbon Nanotube Biocathode. Bioelectrochemistry 2015, 106, 73-76.

(431) Tahar, A. B.; Szymczyk, A.; Tingry, S.; Vadgama, P.; Zelsmann, M.; Tsujumura, S.; Cinquin, P.; Martin, D.; Zebda, A. One-Year stability of Glucose Dehydrogenase Confined in a 3D Carbon Nanotube Electrode with Coated Poly-Methylene Green: Application as Bioanode for a Glucose Biofuel Cell. J. Electroanal. Chem. 2019, DOI:https://doi.org/10.1016/j.jelechem.2019.04.029 https://doi.org/10.1016/j.jelechem.2019.04.029.

(432) Lian, X.; Fang, Y.; Joseph, E.; Wang, Q.; Li, J.; Banerjee, S.; Lollar, C.; Wang, X.; Zhou, H. C. Enzyme-MOF (Metal-Organic Framework) Composites. Chem. Soc. Rev. 2017, 46, 3386-3401.

(433) Patra, S.; Sene, S.; Mousty, C.; Serre, C.; Chausse, A.; Legrand, L.; Steunou, N. Design of Laccase-Metal Organic Framework-Based Bioelectrodes for Biocatalytic Oxygen Reduction Reaction. ACS Appl. Mater. Interfaces 2016, 8, 20012-20022.

(434) Gkaniatsou, E.; Sicard, C.; Ricoux, R.; Mahy, J.-P.; Steunou, N.; Serre, C. Metal-Organic Frameworks: A Novel Host Platform for Enzymatic Catalysis and Detection. Mater. Horiz. 2017, 4, $55-63$. 

Enzyme Encapsulation in Metal-Organic Frameworks for Applications in Catalysis. CrystEngComm 2017, 19, 4082-4091.

(436) Liu, X.; Qi, W.; Wang, Y.; Su, R.; He, Z. A Facile Strategy for Enzyme Immobilization with Highly Stable Hierarchically Porous Metal-Organic Frameworks. Nanoscale 2017, 9, 17561-17570.

(437) Cabana, H.; Jones, J. P.; Agathos, S. N. Preparation and Characterization of Cross-Linked Laccase Aggregates and Their Application to the Elimination of Endocrine Disrupting Chemicals. $J$. Biotechnol. 2007, 132, 23-31.

(438) Barbosa, O.; Ortiz, C.; Berenguer-Murcia, Á.; Torres, R.; Rodrigues, R. C.; Fernandez-Lafuente, R. Glutaraldehyde in Bio-Catalysts Design: a Useful Crosslinker and a Versatile Tool in Enzyme Immobilization. RSC Adv. 2014, 4, 1583-1600.

(439) Xia, H. Q.; Kitazumi, Y.; Shirai, O.; Kano, K. Direct Electron Transfer-Type Bioelectrocatalysis of Peroxidase at Mesoporous Carbon Electrodes and Its Application for Glucose Determination Based on Bienzyme System. Anal. Sci. 2017, 33, 839-844.

(440) Bahar, T. Preparation of a Ferrocene Mediated Bioanode for Biofuel Cells by MWCNTs, Polyethylenimine and Glutaraldehyde: Glucose Oxidase Immobilization and Characterization. Asia-Pac. J. Chem. Eng. 2016, 11, 981-988.

(441) Beneyton, T.; El Harrak, A.; Griffiths, A. D.; Hellwig, P.; Taly, V. Immobilization of CotA, an Extremophilic Laccase from Bacillus subtilis, on Glassy Carbon Electrodes for Biofuel Cell Applications. Electrochem. Commun. 2011, 13, 24-27.

(442) Rengaraj, S.; Kavanagh, P.; Leech, D. A Comparison of Redox Polymer and Enzyme Co-Immobilization on Carbon Electrodes to Provide Membrane-Less Glucose/ $\mathrm{O}_{2}$ Enzymatic Fuel Cells with Improved Power Output and Stability. Biosens. Bioelectron. 2011, 30, 294-299.

(443) Lopez, F.; Zerria, S.; Ruff, A.; Schuhmann, W. An $\mathrm{O}_{2}$ Tolerant Polymer/Glucose Oxidase Based Bioanode as Basis for a Self-Powered Glucose Sensor. Electroanalysis 2018, 30, 1311-1318.

(444) Ruff, A.; Szczesny, J.; Zacarias, S.; Pereira, I. A. C.; Plumeré, N.; Schuhmann, W. Protection and Reactivation of the [NiFeSe] Hydrogenase from Desulfovibrio vulgaris Hildenborough under Oxidative Conditions. ACS Energy Lett. 2017, 2, 964-968.

(445) Pöller, S.; Koster, D.; Schuhmann, W. Stabilizing Redox Polymer Films by Electrochemically Induced Crosslinking. Electrochem. Commun. 2013, 34, 327-330.

(446) Marquitan, M.; Bobrowski, T.; Ernst, A.; Wilde, P.; Clausmeyer, J.; Ruff, A.; Schuhmann, W. Miniaturized Amperometric Glucose Sensors Based on Polymer/ Enzyme Modified Carbon Electrodes in the Sub-Micrometer Scale. J. Electrochem. Soc. 2018, 165, G3008-G3014.

(447) Matijošytė, I.; Arends, I. W. C. E.; de Vries, S.; Sheldon, R. A. Preparation and Use of Cross-Linked Enzyme Aggregates (CLEAs) of Laccases. J. Mol. Catal. B: Enzym. 2010, 62, 142-148.

(448) Dumorne, K.; Cordova, D. C.; Astorga-Elo, M.; Renganathan, P. Extremozymes: A Potential Source for Industrial Applications. J. Microbiol. Biotech. 2017, 27, 649-659.

(449) Zhang, Y. H. P. Production of Biocommodities and Bioelectricity by Cell-Free Synthetic Enzymatic Pathway Biotransformations: Challenges and Opportunities. Biotechnol. Bioeng. 2010, 105, 663-677.

(450) Wang, X. Y.; Li, D.; Watanabe, T.; Shigemori, Y.; Mikawa, T.; Okajima, T.; Mao, L. Q.; 
Ohsaka, T. A Glucose/O ${ }_{2}$ Biofuel Cell Using Recombinant Thermophilic Enzymes. Int. J. Electrochem. Sc. 2012, 7, 1071-1078.

(451) Kwan, P.; McIntosh, C. L.; Jennings, D. P.; Hopkins, R. C.; Chandrayan, S. K.; Wu, C.-H.; Adams, M. W. W.; Jones, A. K. The [NiFe]-Hydrogenase of Pyrococcus furiosus Exhibits a New Type of Oxygen Tolerance. J. Am. Chem. Soc. 2015, 137, 13556-13565.

(452) Zhu, Z; Zhang, Y. H. P. In Vitro Metabolic Engineering of Bioelectricity Generation by the Complete Oxidation of Glucose. Metab. Eng. 2017, 39, 110-116.

(453) Campbell, A. S.; Murata, H.; Carmali, S.; Matyjaszewski, K.; Islam, M. F.; Russell, A. J. Polymer-Based Protein Engineering Grown Ferrocene-Containing Redox Polymers Improve Current Generation in an Enzymatic Biofuel Cell. Biosens. Bioelectron. 2016, 86, 446-453.

(454) Rodrigues, R. C.; Ortiz, C.; Berenguer-Murcia, A.; Torres, R.; Fernandez-Lafuente, R. Modifying Enzyme Activity and Selectivity by Immobilization. Chem. Soc. Rev. 2013, 42, 6290-6307.

(455) Wong, T. S.; Schwaneberg, U. Protein Engineering in Bioelectrocatalysis. Curr. Opin. Biotechnol. 2003 14, 590-596.

(456) Eijsink, V. G. H.; Gaseidnes, S.; Borchert, T. V.; van den Burg, B. Directed Evolution of Enzyme Stability. Biomol. Eng. 2005, 22, 21-30.

(457) Yuhashi, N.; Tomiyama, M.; Okuda, J.; Igarashi, S.; Ikebukuro, K.; Sode, K. Development of a Novel Glucose Enzyme Fuel Cell System Employing Protein Engineered PQQ Glucose Dehydrogenase. Biosens. Bioelectron. 2005, 20, 2145-2150.

(458) Tanaka, S.; Igarashi, S.; Ferri, S.; Sode, K. Increasing Stability of Water-Soluble PQQ Glucose Dehydrogenase by Increasing Hydrophobic Interaction at Dimeric Interface. BMC Biochem. 2005, 6, 1 .

(459) Mate, Diana M.; Gonzalez-Perez, D.; Falk, M.; Kittl, R.; Pita, M.; De Lacey, Antonio L.; Ludwig, R.; Shleev, S.; Alcalde, M. Blood Tolerant Laccase by Directed Evolution. Chem. Biol. 2013, 20, 223-231.

(460) Pereira, A. R.; Luz, R. A. S.; Lima, F. C. D. A.; Crespilho, F. N. Protein Oligomerization Based on Brønsted Acid Reaction. ACS Catal. 2017, 7, 3082-3088.

(461) Campbell, E.; Meredith, M.; Minteer, S. D.; Banta, S. Enzymatic Biofuel Cells Utilizing a Biomimetic Cofactor. Chem. Commun. 2012, 48, 1898-1900.

(462) Chen, H.; Zhu, Z. G.; Huang, R.; Zhang, Y. H. P. Coenzyme Engineering of a Hyperthermophilic 6-Phosphogluconate Dehydrogenase from $\mathrm{NADP}^{+}$to $\mathrm{NAD}^{+}$with Its Application to Biobatteries. Sci. Rep. 2016, 6, 36311.

(463) Lee, S. Y.; Choi, J. H.; Xu, Z. Microbial Cell-Surface Display. Trends Biotechnol. 2003, 21, 45-52.

(464) Van Bloois, E.; Winter, R. T.; Kolmar, H.; Fraaije, M. W. Decorating Microbes: Surface Display of Proteins on Escherichia coli. Trends Biotechnol. 2011, 29, 79-86.

(465) Shimazu, M.; Mulchandani, A.; Chen, W. Cell Surface Display of Organophosphorus Hydrolase Using Ice Nucleation Protein. Biotechnol. Prog. 2001, 17, 76-80.

(466) Yang, C.; Zhu, Y.; Yang, J.; Liu, Z.; Qiao, C.; Mulchandani, A.; Chen, W. Development of an Autofluorescent Whole-Cell Biocatalyst by Displaying Dual Functional Moieties on Escherichia coli Cell Surfaces and Construction of a Coculture with Organophosphate-Mineralizing Activity. Appl. Microbiol. Biotechnol. 2008, 74, 7733-7739. 

Pseudomonas and Burkholderia Lipases Using a Translocator Domain of EstA Autotransporter on the Cell Surface of Escherichia coil. J. Biotechnol. 2010, 146, 126-129.

(468) Samuelson, P.; Gunneriusson, E.; Nygren, P. A.; Stahl, S. Display of Proteins on Bacteria. J. Biotechnol. 2002, 96, 129-154.

(469) Daugherty, P. S. Protein Engineering with Bacterial Display. Curr. Opin. Struct. Biol. 2007, $17,474-480$.

(470) Jahns, A. C.; Rehm, B. H. A. Relevant uses of surface proteins - display on self-organized biological structures. Microb. Biotechnol. 2012, 5, 188-202.

(471) Tang, X.; Liang, B.; Yi, T.; Manco, G.; IlariaPalchetti; Liu, A. Cell Surface Display of Organophosphorus Hydrolase for Sensitive Spectrophotometric Detection of $P$-Nitrophenol Substituted Organophosphates. Enzyme Microb. Technol. 2014, 55, 107-112.

(472) Lee, J.-S.; Shin, K.-S.; Pan, J.-G.; Kim, C.-J. Surface-Displayed Viral Antigens on Salmonella carrier Vaccine. Nat. Biotech. 2000, 18, 645-648.

(473) Binder, U.; Matschiner, G.; Theobald, I.; Skerra, A. High-Throughput Sorting of an Anticalin Library via EspP-Mediated Functional Display on the Escherichia coli Cell Surface. J. Mol. Biol. 2010, 400, 783-802.

(474) Saleem, M.; Brim, H.; Hussain, S.; Arshad, M.; Leigh, M. B.; Zia ul, h. Perspectives on Microbial Cell Surface Display in Bioremediation. Biotechnol. Adv. 2008, 26, 151-161.

(475) Jin, Z.; Han, S.-Y.; Zhang, L.; Zheng, S.-P.; Wang, Y.; Lin, Y. Combined Utilization of Lipase-Displaying Pichia pastoris Whole-Cell Biocatalysts to Improve Biodiesel Production in Co-Solvent Media. Bioresour. Technol. 2013, 130, 102-109.

(476) Liang, B.; Wang, G.; Yan, L.; Ren, H.; Feng, R.; Xiong, Z.; Liu, A. Functional Cell Surface Displaying of Acetylcholinesterase for Spectrophotometric Sensing Organophosphate Pesticide. Sens. Actuat. B: Chem. 2019, 279, 483-489.

(477) Amir, L.; Carnally, S. A.; Rayo, J.; Rosenne, S.; Melamed Yerushalmi, S.; Schlesinger, O.; Meijler, M. M.; Alfonta, L. Surface Display of a Redox Enzyme and Its Site-Specific Wiring to Gold Electrodes. J. Am. Chem. Soc. 2013, 135, 70-73.

(478) Simon Fishilevich; Liron Amir; Yearit Fridman; Amir Aharoni; Alfonta, L. Surface Display of Redox Enzymes in Microbial Fuel Cells. J. Am. Chem. Soc. 2009, 131, 12052-12053.

(479) Alfonta, L. Genetically Engineered Microbial Fuel Cells. Electroanalysis 2010, 22, 822-831.

(480) Osman, M. H.; Shah, A. A.; Walsh, F. C. Recent Progress and Continuing Challenges in Bio-Fuel Cells. Part I: Enzymatic Cells. Biosens. Bioelectron. 2011, 26, 3087-3102.

(481) Xie, X.; Ye, M.; Hsu, P.-C.; Liu, N.; Criddle, C. S.; Cui, Y. Microbial Battery for Efficient Energy Recovery. Proc. Natl. Acad. Sci. 2013, 110, 15925-15930.

(482) Herrero-Hernandez, E.; Smith, T. J.; Akid, R. Electricity Generation from Wastewaters with Starch as Carbon Source Using a Mediatorless Microbial Fuel Cell. Biosens. Bioelectron. 2013, 39, 194-198.

(483) Liang, B.; Li, L.; Mascin, M.; Liu, A. Construction of Xylose Dehydrogenase Displayed on the Surface of Bacteria Using Ice Nucleation Protein for Sensitive D-Xylose Detection. Anal. Chem. 2012, 84, 275-282.

(484) Liang, B.; Li, L.; Tang, X.; Lang, Q.; Wang, H.; Li, F.; Shi, J.; Shen, W.; Palchetti, I.; 
Mascini, M.et al. Microbial Surface Display of Glucose Dehydrogenase for Amperometric Glucose Biosensor. Biosens. Bioelectron. 2013, 45, 19-24.

(485) Liang, B.; Lang, Q.; Tang, X.; Liu, A. Simultaneously Improving Stability and Specificity of Cell Surface Displayed Glucose Dehydrogenase Mutants to Construct Whole-Cell Biocatalyst for Glucose Biosensor Application. Bioresour. Technol. 2013, 147, 492-498.

(486) Liang, B.; Zhang, S.; Lang, Q.; Song, J.; Han, L.; Liu, A. Amperometric L-Glutamate Biosensor Based on Bacterial Cell-Surface Displayed Glutamate Dehydrogenase. Anal. Chim. Acta 2015, 884, 83-89.

(487) Liu, A.; Liang, B.; Feng, R. Microbial Surface Displaying Formate Dehydrogenase and Its Application in Optical Detection of Formate. Enzyme Microb. Technol. 2016, 91, 59-65.

(488) Wang, H.; Lang, Q.; Li, L.; Liang, B.; Tang, X.; Kong, L.; Mascini, M.; Liu, A. Yeast Surface Displaying Glucose Oxidase as Whole-Cell Biocatalyst: Construction, Characterization, and Its Electrochemical Glucose Sensing Application. Anal. Chem. 2013, 85, 6107-6112.

(489) Lang, Q.; Wang, F.; Yin, L.; Liu, M.; Petrenko, V. A.; Liu, A. Specific Probe Selection from Landscape Phage Display Library and Its Application in Enzyme-Linked Immunosorbent Assay of Free Prostate-Specific Antigen. Anal. Chem. 2014, 86, 2767-2774.

(490) Han, L.; Liu, A. Novel Cell-Inorganic Hybrid Catalytic Interfaces with Enhanced Enzymatic Activity and Stability for Sensitive Biosensing of Paraoxon. ACS Appl. Mater. Interfaces 2017, 9, 6894-6901.

(491) Han, L.; Xia, H.; Yin, L.; Petrenko, V. A.; Liu, A. Selected Landscape Phage Probe as Selective Recognition Interface for Sensitive Total Prostate-Specific Antigen Immunosensor. Biosens. Bioelectron. 2018, 106, 1-6.

(492) Xia, L.; Liang, B.; Li, L.; Tang, X.; Palchetti, I.; Mascini, M.; Liu, A. Direct Energy Conversion from Xylose Using Xylose Dehydrogenase Surface Displayed Bacteria Based Enzymatic Biofuel Cell. Biosens. Bioelectron. 2013, 44, 160-163.

(493) Feng, R.; Liang, B.; Hou, C.; Han, D.; Han, L.; Lang, Q.; Liu, A.; Han, L. Rational Design of Xylose Dehydrogenase for Improved Thermostability and Its Application in Development of Efficient Enzymatic Biofuel Cell. Enzyme Microb. Technol. 2016, 84, 78-85.

(494) Lang, Q.; Yin, L.; Shi, J.; Li, L.; Xia, L.; Liu, A. Co-Immobilization of Glucoamylase and Glucose Oxidase for Electrochemical Sequential Enzyme Electrode for Starch Biosensor and Biofuel Cell. Biosens. Bioelectron. 2014, 51, 158-163.

(495) Yamamoto, K.; Matsumoto, T.; Shimada, S.; Tanaka, T.; Kondo, A. Starchy Biomass-Powered Enzymatic Biofuel Cell Based on Amylases and Glucose Oxidase Multi-Immobilized Bioanode. New Biotech. 2013, 30, 531-535.

(496) Bahartan, K.; Amir, L.; Israel, A.; Lichtenstein, R. G.; Alfonta, L. In situ Fuel Processing in a Microbial Fuel Cell. ChemSusChem 2012, 5, 1820-1825.

(497) Dijkman, W. P.; de Gonzalo, G.; Mattevi, A.; Fraaije, M. W. Flavoprotein Oxidases: Classification and Applications. Appl. Microbiol. Biotechnol. 2013, 97, 5177-5188.

(498) Gadda, G. Oxygen Activation in Flavoprotein Oxidases: The Importance of Being Positive. Biochemistry 2012, 51, 2662-2669.

(499) Prévoteau, A.; Mano, N. How the Reduction of $\mathrm{O}_{2}$ on Enzymes and/or Redox Mediators Affects the Calibration Curve of "Wired" Glucose Oxidase and Glucose Dehydrogenase Biosensors. 
Electrochim. Acta 2013, 112, 318-326.

(500) Wilson, R.; Turner, A. P. F. Glucose Oxidase: An Ideal Enzyme. Biosens. Bioelectron. 1992, 7, 165-185.

(501) Brusova, Z.; Gorton, L.; Magner, E. Comment on "Direct Electrochemistry and Electrocatalysis of Heme Proteins Entrapped in Agarose Hydrogel Films in Room-Temperature Ionic Liquids”. Langmuir 2006, 22, 11453-11455.

(502) Scodeller, P.; Carballo, R.; Szamocki, R.; Levin, L.; Forchiassin, F.; Calvo, E. J. Layer-by-Layer Self-Assembled Osmium Polymer-Mediated Laccase Oxygen Cathodes for Biofuel Cells: The Role of Hydrogen Peroxide. J. Am. Chem. Soc. 2010, 132, 11132-11140.

(503) Milton, R. D.; Giroud, F.; Thumser, A. E.; Minteer, S. D.; Slade, R. C. T. Bilirubin Oxidase Bioelectrocatalytic Cathodes: The Impact of Hydrogen Peroxide. Chem. Commun. 2014, 50, 94-96.

(504) Willner, I.; Arad, G.; Katz, E. A Biofuel Cell Based on Pyrroloquinoline Quinone and Microperoxidase-11 Monolayer-Functionalized Electrodes. Bioelectrochem. Bioenerg. 1998, 44, 209-214.

(505) Katz, E.; Lioubashevski, O.; Willner, I. Magnetic Field Effects on Bioelectrocatalytic Reactions of Surface-Confined Enzyme Systems: Enhanced Performance of Biofuel Cells. J. Am. Chem. Soc. 2005, 127, 3979-3988.

(506) Koushanpour, A.; Gamella, M.; Katz, E. A Biofuel Cell Based on Biocatalytic Reactions of Lactate on Both Anode and Cathode Electrodes - Extracting Electrical Power from Human Sweat. Electroanalysis 2017, 29, 1602-1611.

(507) Ruff, A.; Pinyou, P.; Nolten, M.; Conzuelo, F.; Schuhmann, W. A Self-Powered Ethanol Biosensor. ChemElectroChem 2017, 4, 890-897.

(508) Cheng, F; Zhu, L.; Schwaneberg, U. Directed Evolution 2.0: Improving and Deciphering Enzyme Properties. Chem. Commun. 2015, 51, 9760-9772.

(509) Klinman, J. P. How Do Enzymes Activate Oxygen without Inactivating Themselves? Acc. Chem. Res. 2007, 40, 325-333.

(510) Petrović, D.; Frank, D.; Kamerlin, S. C. L.; Hoffmann, K.; Strodel, B. Shuffling Active Site Substate Populations Affects Catalytic Activity: The Case of Glucose Oxidase. ACS Catal. 2017, 7, 6188-6197.

(511) Roth, J. P.; Klinman, J. P. Catalysis of Electron Transfer During Activation of $\mathrm{O}_{2}$ by the Flavoprotein Glucose Oxidase. Proc. Natl. Acad. Sci. 2003, 100, 62-67.

(512) Arango Gutierrez, E.; Mundhada, H.; Meier, T.; Duefel, H.; Bocola, M.; Schwaneberg, U. Reengineered Glucose Oxidase for Amperometric Glucose Determination in Diabetes Analytics. Biosens. Bioelectron. 2013, 50, 84-90.

(513) Horaguchi, Y.; Saito, S.; Kojima, K.; Tsugawa, W.; Ferri, S.; Sode, K. Construction of Mutant Glucose Oxidases with Increased Dye-Mediated Dehydrogenase Activity. Int. J. Mol. Sci. 2012, 13.

(514) Horaguchi, Y.; Saito, S.; Kojima, K.; Tsugawa, W.; Ferri, S.; Sode, K. Engineering Glucose Oxidase to Minimize the Influence of Oxygen on Sensor Response. Electrochim. Acta 2014, 126, 158-161.

(515) Suraniti, E.; Courjean, O.; Gounel, S.; Tremey, E.; Mano, N. Uncovering and Redesigning a Key Amino Acid of Glucose Oxidase for Improved Biotechnological Applications. Electroanalysis 
2013, 25, 606-611.

(516) Tremey, E.; Stines-Chaumeil, C.; Gounel, S.; Mano, N. Designing an $\mathrm{O}_{2}$-Insensitive Glucose

Oxidase for Improved Electrochemical Applications. ChemElectroChem 2017, 4, 2520-2526.

(517) Hiraka, K.; Kojima, K.; Lin, C.-E.; Tsugawa, W.; Asano, R.; La Belle, J. T.; Sode, K. Minimizing the Effects of Oxygen Interference on L-Lactate Sensors by a Single Amino Acid Mutation in Aerococcus Viridans L-Lactate Oxidase. Biosens. Bioelectron. 2018, 103, 163-170.

(518) Gorton, L.; Domı; x; nguez, E. Electrocatalytic Oxidation of $\mathrm{NAD}(\mathrm{P}) \mathrm{H}$ at Mediator Modified Electrodes. Rev. Mol. Biotechnol. 2002, 82, 371-392.

(519) Durand, F.; Stines-Chaumeil, C.; Flexer, V.; André, I.; Mano, N. Designing a Highly Active Soluble PQQ-Glucose Dehydrogenase for Efficient Glucose Biosensors and Biofuel Cells. Biochem. Biophys. Res. Commun. 2010, 402, 750-754.

(520) Schubart, I. W.; Göbel, G.; Lisdat, F. A Pyrroloquinolinequinone-Dependent Glucose Dehydrogenase (PQQ-GDH)-Electrode with Direct Electron Transfer Based on Polyaniline Modified Carbon Nanotubes for Biofuel Cell Application. Electrochim. Acta 2012, 82, 224-232.

(521) Giroud, F.; Milton, R. D.; Tan, B.-X.; Minteer, S. D. Simplifying Enzymatic Biofuel Cells: Immobilized Naphthoquinone as a Biocathodic Orientational Moiety and Bioanodic Electron Mediator. ACS Catal. 2015, 5, 1240-1244.

(522) Tanne, C.; Göbel, G.; Lisdat, F. Development of a (PQQ)-GDH-Anode Based on MWCNT-Modified Gold and its Application in a Glucose/ $\mathrm{O}_{2}-$ Biofuel Cell. Biosens. Bioelectron. 2010, $26,530-535$.

(523) Flexer, V.; Durand, F.; Tsujimura, S.; Mano, N. Efficient Direct Electron Transfer of PQQ-Glucose Dehydrogenase on Carbon Cryogel Electrodes at Neutral pH. Anal. Chem. 2011, 83, 5721-5727.

(524) Xiao, X.; Conghaile, P. Ó.; Leech, D.; Ludwig, R.; Magner, E. An Oxygen-Independent and Membrane-Less Glucose Biobattery/Supercapacitor Hybrid Device. Biosens. Bioelectron. 2017, 98, 421-427.

(525) Milton, R. D.; Giroud, F.; Thumser, A. E.; Minteer, S. D.; Slade, R. C. T. Glucose Oxidase Progressively Lowers Bilirubin Oxidase Bioelectrocatalytic Cathode Performance in Single-Compartment Glucose/Oxygen Biological Fuel Cells. Electrochim. Acta 2014, 140, 59-64.

(526) Yehezkeli, O.; Tel-Vered, R.; Raichlin, S.; Willner, I. Nano-engineered Flavin-Dependent Glucose Dehydrogenase/Gold Nanoparticle-Modified Electrodes for Glucose Sensing and Biofuel Cell Applications. ACS Nano 2011, 5, 2385-2391.

(527) Tsujimura, S.; Kojima, S.; Kano, K.; Ikeda, T.; Sato, M.; Sanada, H.; Omura, H. Novel FAD-Dependent Glucose Dehydrogenase for a Dioxygen-Insensitive Glucose Biosensor. Biosci. Biotechnol. Biochem. 2006, 70, 654-659.

(528) Okuda-Shimazaki, J.; Kakehi, N.; Yamazaki, T.; Tomiyama, M.; Sode, K. Biofuel Cell System Employing Thermostable Glucose Dehydrogenase. Biotechnol. Lett. 2008, 30, 1753-1758.

(529) Zafar, M. N.; Beden, N.; Leech, D.; Sygmund, C.; Ludwig, R.; Gorton, L. Characterization of Different FAD-Dependent Glucose Dehydrogenases for Possible Use in Glucose-Based Biosensors and Biofuel Cells. Anal. Bioanal. Chem. 2012, 402, 2069-2077.

(530) Milton, R. D.; Giroud, F.; Thumser, A. E.; Minteer, S. D.; Slade, R. C. T. Hydrogen Peroxide 
Produced by Glucose Oxidase Affects the Performance of Laccase Cathodes in Glucose/Oxygen Fuel Cells: FAD-Dependent Glucose Dehydrogenase as a Replacement. Phys. Chem. Chem. Phys. 2013, 15, 19371-19379.

(531) Milton, R. D.; Lim, K.; Hickey, D. P.; Minteer, S. D. Employing FAD-Dependent Glucose Dehydrogenase Within a Glucose/Oxygen Enzymatic Fuel Cell Operating in Human Serum. Bioelectrochemistry 2015, 106, Part A, 56-63. Osadebe, I.; Leech, D. Effect of Multi-Walled Carbon Nanotubes on Glucose Oxidation by

Glucose Oxidase or a Flavin-Dependent Glucose Dehydrogenase in Redox-Polymer-Mediated Enzymatic Fuel Cell Anodes. ChemElectroChem 2014, 1, 1988-1993.

(533) Iwasa, H.; Hiratsuka, A.; Yokoyama, K.; Uzawa, H.; Orihara, K.; Muguruma, H. Thermophilic Talaromyces emersonii Flavin Adenine Dinucleotide-Dependent Glucose Dehydrogenase Bioanode for Biosensor and Biofuel Cell Applications. ACS Omega 2017, 2, 1660-1665.

(534) Tan, T. C.; Spadiut, O.; Wongnate, T.; Sucharitakul, J.; Krondorfer, I.; Sygmund, C.; Haltrich, D.; Chaiyen, P.; Peterbauer, C. K.; Divne, C. The 1.6 A Crystal Structure of Pyranose Dehydrogenase from Agaricus meleagris Rationalizes Substrate Specificity and Reveals a Flavin Intermediate. PLOS ONE 2013, 8, e53567.

(535) Zafar, M. N.; Tasca, F.; Boland, S.; Kujawa, M.; Patel, I.; Peterbauer, C. K.; Leech, D.; Gorton, L. Wiring of Pyranose Dehydrogenase with Osmium Polymers of Different Redox Potentials. Bioelectrochemistry 2010, 80, 38-42.

(536) Yakovleva, M. E.; Killyéni, A.; Seubert, O.; Ó Conghaile, P.; MacAodha, D.; Leech, D.; Gonaus, C.; Popescu, I. C.; Peterbauer, C. K.; Kjellström, S.et al. Further Insights into the Catalytical Properties of Deglycosylated Pyranose Dehydrogenase from Agaricus meleagris Recombinantly Expressed in Pichia pastoris. Anal. Chem. 2013, 85, 9852-9858.

(537) Yakovleva, M. E.; Gonaus, C.; Schropp, K.; Oconghaile, P.; Leech, D.; Peterbauer, C. K.; Gorton, L. Engineering of Pyranose Dehydrogenase for Application to Enzymatic Anodes in Biofuel Cells. Phys. Chem. Chem. Phys. 2015, 17, 9074-9081.

(538) Sygmund, C.; Kittl, R.; Volc, J.; Halada, P.; Kubátová, E.; Haltrich, D.; Peterbauer, C. K. Characterization of Pyranose Dehydrogenase from Agaricus meleagris and Its Application in the C-2 Specific Conversion of D-Galactose. J. Biotechnol. 2008, 133, 334-342.

(539) Wait, A. F.; Parkin, A.; Morley, G. M.; dos Santos, L.; Armstrong, F. A. Characteristics of Enzyme-Based Hydrogen Fuel Cells Using an Oxygen-Tolerant Hydrogenase as the Anodic Catalyst. $J$. Phys. Chem. C 2010, 114, 12003-12009.

(540) Plumeré, N.; Rüdiger, O.; Oughli, A. A.; Williams, R.; Vivekananthan, J.; Pöller, S.; Schuhmann, W.; Lubitz, W. A Redox Hydrogel Protects Hydrogenase from High-Potential Deactivation and Oxygen Damage. Nat. Chem. 2014, 6, 822.

(541) Fourmond, V.; Stapf, S.; Li, H.; Buesen, D.; Birrell, J.; Rüdiger, O.; Lubitz, W.; Schuhmann, W.; Plumeré, N.; Léger, C. Mechanism of Protection of Catalysts Supported in Redox Hydrogel Films. J. Am. Chem. Soc. 2015, 137, 5494-5505.

(542) Oughli, A. A.; Conzuelo, F.; Winkler, M.; Happe, T.; Lubitz, W.; Schuhmann, W.; Rüdiger, O.; Plumeré, N. A Redox Hydrogel Protects the $\mathrm{O}_{2}$-Sensitive [FeFe]-Hydrogenase from 
Chlamydomonas reinhardtii from Oxidative Damage. Angew. Chem. Int. Ed. 2015, 54, 12329-12333.

(543) Ruff, A.; Szczesny, J.; Marković, N.; Conzuelo, F.; Zacarias, S.; Pereira, I. A. C.; Lubitz, W.; Schuhmann, W. A Fully Protected Hydrogenase/Polymer-Based Bioanode for High-Performance Hydrogen/Glucose Biofuel Cells. Nat. Commun. 2018, 9, 3675.

(544) Lopez, F.; Ma, S.; Ludwig, R.; Schuhmann, W.; Ruff, A. A Polymer Multilayer Based Amperometric Biosensor for the Detection of Lactose in the Presence of High Concentrations of Glucose. Electroanalysis 2017, 29, 154-161.

(545) Barfidokht, A.; Gooding, J. J. Approaches Toward Allowing Electroanalytical Devices to be Used in Biological Fluids. Electroanalysis 2014, 26, 1182-1196.

(546) Wisniewski, N.; Reichert, M. Methods for Reducing Biosensor Membrane Biofouling. Coll. Surf. B: Biointerfaces 2000, 18, 197-219.

(547) Trouillon, R.; Combs, Z.; Patel, B. A.; O’Hare, D. Comparative Study of the Effect of Various Electrode Membranes on Biofouling and Electrochemical Measurements. Electrochem. Commun. 2009, 11, 1409-1413.

(548) Wu, H.; Lee, C.-J.; Wang, H.; Hu, Y.; Young, M.; Han, Y.; Xu, F.-J.; Cong, H.; Cheng, G. Highly Sensitive and Stable Zwitterionic Poly(Sulfobetaine-3,4-Ethylenedioxythiophene) (PSBEDOT) Glucose Biosensor. Chem. Sci. 2018, 9, 2540-2546.

(549) Daggumati, P.; Matharu, Z.; Wang, L.; Seker, E. Biofouling-Resilient Nanoporous Gold Electrodes for DNA Sensing. Anal. Chem. 2015, 87, 8618-8622.

(550) El Ichi-Ribault, S.; Alcaraz, J.-P.; Boucher, F.; Boutaud, B.; Dalmolin, R.; Boutonnat, J.; Cinquin, P.; Zebda, A.; Martin, D. K. Remote Wireless Control of an Enzymatic Biofuel Cell Implanted in a Rabbit for 2 Months. Electrochim. Acta 2018, 269, 360-366.

(551) Falk, M.; Andoralov, V.; Blum, Z.; Sotres, J.; Suyatin, D. B.; Ruzgas, T.; Arnebrant, T.; Shleev, S. Biofuel Cell as a Power Source for Electronic Contact Lenses. Biosens. Bioelectron. 2012, 37, 38-45.

(552) Falk, M.; Pankratov, D.; Lindh, L.; Arnebrant, T.; Shleev, S. Miniature Direct Electron Transfer Based Enzymatic Fuel Cell Operating in Human Sweat and Saliva. Fuel Cells 2014, 14, 1050-1056.

(553) Göbel, G.; Beltran, M. L.; Mundhenk, J.; Heinlein, T.; Schneider, J.; Lisdat, F. Operation of a Carbon Nanotube-Based Glucose/Oxygen Biofuel Cell in Human Body Liquids-Performance Factors and Characteristics. Electrochim. Acta 2016, 218, 278-284.

(554) Bollella, P.; Fusco, G.; Stevar, D.; Gorton, L.; Ludwig, R.; Ma, S.; Boer, H.; Koivula, A.; Tortolini, C.; Favero, G.et al. A Glucose/Oxygen Enzymatic Fuel Cell Based on Gold Nanoparticles modified Graphene Screen-Printed Electrode. Proof-of-Concept in Human Saliva. Sens. Actuat. B: Chem. 2018, 256, 921-930.

(555) Falk, M.; Andoralov, V.; Silow, M.; Toscano, M. D.; Shleev, S. Miniature Biofuel Cell as a Potential Power Source for Glucose-Sensing Contact Lenses. Anal. Chem. 2013, 85, 6342-6348.

(556) Xiao, X.; Siepenkoetter, T.; Conghaile, P. Ó.; Leech, D.; Magner, E. Nanoporous Gold-Based Biofuel Cells on Contact Lenses. ACS Appl. Mater. Interfaces 2018, 10, 7107-7116.

(557) Kai, H.; Yamauchi, T.; Ogawa, Y.; Tsubota, A.; Magome, T.; Miyake, T.; Yamasaki, K.; Nishizawa, M. Accelerated Wound Healing on Skin by Electrical Stimulation with a Bioelectric Plaster. Adv. Healthc. Mater. 2017, 6, 1700465. 

Organic Transdermal Iontophoresis Patch with Built-in Biofuel Cell. Adv. Healthc. Mater. 2015, 4, 506-510.

(559) Kizling, M.; Biedul, P.; Zabost, D.; Stolarczyk, K.; Bilewicz, R. Application of Hydroxyethyl Methacrylate and Ethylene Glycol Methacrylate Phosphate Copolymer as Hydrogel Electrolyte in Enzymatic Fuel Cell. Electroanalysis 2016, 28, 2444-2451.

(560) Xiao, X.; Magner, E. A Quasi-Solid-State and Self-Powered Biosupercapacitor Based on Flexible Nanoporous Gold Electrodes. Chem. Commun. 2018, 54, 5823-5826.

(561) Gamella, M.; Koushanpour, A.; Katz, E. Biofuel Cells - Activation of Micro- and Macro-Electronic Devices. Bioelectrochemistry 2018, 119, 33-42.

(562) Shleev, S.; Bergel, A.; Gorton, L. Biological Fuel Cells: Divergence of Opinion. Bioelectrochemistry 2015, 106, 1-2.

(563) Mark, A. G.; Suraniti, E.; Roche, J.; Richter, H.; Kuhn, A.; Mano, N.; Fischer, P. On-Chip Enzymatic Microbiofuel Cell-Powered Integrated Circuits. Lab Chip 2017, 17, 1761-1768.

(564) Hu, H.; Islam, T.; Kostyukova, A.; Ha, S.; Gupta, S. From Battery Enabled to Natural Harvesting: Enzymatic BioFuel Cell Assisted Integrated Analog Front-End in $130 \mathrm{~nm}$ CMOS for Long-Term Monitoring. IEEE Trans. Circuits Syst. I, Reg. Papers 2018, 66, 534-545.

(565) Winkler, W.; Nehter, P. In Modeling Solid Oxide Fuel Cells: Methods, Procedures and Techniques; Springer Netherlands: Dordrecht, 2008.

(566) Alberty, R. A. Calculating Apparent Equilibrium Constants of Enzyme-Catalyzed Reactions at pH 7. Biochem. Mol. Biol. Educ. 2000, 28, 12-17.

(567) Bard, A. J.; Faulkner, L. R. Electrochemical Methods-Fundamental and Applications. John Wiley \& Sons Inc., New York (2001) 2001.

(568) Umasankar, Y.; Brooks, D. B.; Brown, B.; Zhou, Z.; Ramasamy, R. P. Three Dimensional Carbon Nanosheets as a Novel Catalyst Support for Enzymatic Bioelectrodes. Adv. Energy Mater. 2014, 4, 1301306.

(569) Xiao, X.; Magner, E. A Biofuel Cell in Non-Aqueous Solution. Chem. Commun. 2015, 51, 13478-13480.

(570) Richardson, D. J. Bacterial Respiration: A Flexible Process for a Changing Environment. Microbiology 2000, 146, 551-571.

(571) Rabaey, K.; Verstraete, W. Microbial Fuel Cells: Novel Biotechnology for Energy Generation. Trends Biotechnol. 2005, 23, 291-298.

(572) Katz, E.; Schlereth, D. D.; Schmidt, H.-L. Electrochemical Study of Pyrroloquinoline Quinone Covalently Immobilized as a Monolayer onto a Cystamine-Modified Gold Electrode. $J$. Electroanal. Chem. 1994, 367, 59-70.

(573) Coman, V.; Vaz-Dominguez, C.; Ludwig, R.; Harreither, W.; Haltrich, D.; De Lacey, A. L.; Ruzgas, T.; Gorton, L.; Shleev, S. A Membrane-, Mediator-, Cofactor-Less Glucose/Oxygen Biofuel Cell. Phys. Chem. Chem. Phys. 2008, 10, 6093-6096.

(574) Gallaway, J. W.; Calabrese Barton, S. A. Kinetics of Redox Polymer-Mediated Enzyme Electrodes. J. Am. Chem. Soc. 2008, 130, 8527-8536.

(575) Kavanagh, P.; Leech, D. Mediated Electron Transfer in Glucose Oxidising Enzyme 
Electrodes for Application to Biofuel Cells: Recent Progress and Perspectives. Phys. Chem. Chem. Phys. 2013, 15, 4859-4869.

(576) Heller, A. Electrical Connection of Enzyme Redox Centers to Electrodes. J. Phys. Chem. 1992, 96, 3579-3587.

(577) Heller, A.; Feldman, B. Electrochemical Glucose Sensors and Their Applications in Diabetes Management. Chem. Rev. 2008, 108, 2482-2505.

(578) Soukharev, V.; Mano, N.; Heller, A. A Four-Electron $\mathrm{O}_{2}$-Electroreduction Biocatalyst Superior to Platinum and a Biofuel Cell Operating at 0.88 V. J. Am. Chem. Soc. 2004, 126, 8368-8369.

(579) Hickey, D. P.; Halmes, A. J.; Schmidtke, D. W.; Glatzhofer, D. T. Electrochemical Characterization of Glucose Bioanodes Based on Tetramethylferrocene-Modified Linear Poly(ethylenimine). Electrochim. Acta 2014, 149, 252-257.

(580) Liu, A.; Anzai, J.-i. Ferrocene-Containing Polyelectrolyte Multilayer Films: Effects of Electrochemically Inactive Surface Layers on the Redox Properties. Langmuir 2003, 19, 4043-4046.

(581) Tapia, C.; Milton, R. D.; Pankratova, G.; Minteer, S. D.; Åkerlund, H.-E.; Leech, D.; De Lacey, A. L.; Pita, M.; Gorton, L. Wiring of Photosystem I and Hydrogenase on an Electrode for Photoelectrochemical $\mathrm{H}_{2}$ Production by using Redox Polymers for Relatively Positive Onset Potential. ChemElectroChem 2017, 4, 90-95.

(582) Pinyou, P.; Ruff, A.; Pöller, S.; Alsaoub, S.; Leimkühler, S.; Wollenberger, U.; Schuhmann, W. Wiring of the Aldehyde Oxidoreductase PaoABC to Electrode Surfaces via Entrapment in Low Potential Phenothiazine-Modified Redox Polymers. Bioelectrochemistry 2016, 109, 24-30.

(583) Abdellaoui, S.; Milton, R. D.; Quah, T.; Minteer, S. D. NAD-Dependent Dehydrogenase Bioelectrocatalysis: The Ability of a Naphthoquinone Redox Polymer to Regenerate NAD. Chem. Commun. 2016, 52, 1147-1150.

(584) Hou, C.; Lang, Q.; Liu, A. Tailoring 1,4-Naphthoquinone with Electron-Withdrawing Group: Toward Developing Redox Polymer and FAD-GDH Based Hydrogel Bioanode for Efficient Electrocatalytic Glucose Oxidation. Electrochim. Acta 2016, 211, 663-670.

(585) Liu, A.; Anzai, J.-i.; Wang, J. Multilayer Assembly of Calf Thymus DNA and Poly(4-Vinylpyridine) (PVP) Derivative Bearing $\left[\mathrm{Os}(\mathrm{bpy})_{2} \mathrm{Cl}\right]^{2+}$ : Redox Behavior within DNA Film. Bioelectrochem. 2005, 67, 1-6.

(586) Liu, A.; Anzai, J.-i. Use of Polymeric Indicator for Electrochemical DNA Sensors: Poly(4-Vinylpyridine) Derivative Bearing [Os(5,6-Dimethyl-1,10-Phenanthroline)2Cl] ${ }^{2+}$. Anal. Chem. 2004, 76, 2975-2980.

(587) Pinyou, P.; Ruff, A.; Pöller, S.; Ma, S.; Ludwig, R.; Schuhmann, W. Design of an Os Complex-Modified Hydrogel with Optimized Redox Potential for Biosensors and Biofuel Cells. Chem. Eur. J. 2016, 22, 5319-5326.

(588) Guschin, D. A.; Castillo, J.; Dimcheva, N.; Schuhmann, W. Redox Electrodeposition Polymers: Adaptation of the Redox Potential of Polymer-Bound Os Complexes for Bioanalytical Applications. Anal. Bioanal. Chem. 2010, 398, 1661-1673.

(589) Bartlett, P. N.; Pratt, K. F. E. Theoretical Treatment of Diffusion and Kinetics in Amperometric Immobilized Enzyme Electrodes Part I: Redox Mediator Entrapped Within the Film. $J$. Electroanal. Chem. 1995, 397, 61-78.

(590) Zafar, M. N.; Wang, X.; Sygmund, C.; Ludwig, R.; Leech, D.; Gorton, L. Electron-Transfer 
Studies with a New Flavin Adenine Dinucleotide Dependent Glucose Dehydrogenase and Osmium Polymers of Different Redox Potentials. Anal. Chem. 2012, 84, 334-341.

(591) Liu, A.; Anzai, J.-i. A Poly(4-Vinylpyridine) Derivative Bearing Os(5,6-Dmphen $)_{2} \mathrm{Cl}$ (5,6-Dmphen=5,6-Dimethyl-1,10-Phenanthroline): a Novel Electrochemical Indicator for Detecting DNA Hybridization. Mater. Sci. Eng. C 2004, 24, 503-505.

(592) Pinyou, P.; Pöller, S.; Chen, X.; Schuhmann, W. Optimization of Os-Complex Modified Redox Polymers for Improving Biocatalysis of PQQ-sGDH Based Electrodes. Electroanalysis 2015, 27, 200-208.

(593) Oughli, A. A.; Vélez, M.; Birrell, J. A.; Schuhmann, W.; Lubitz, W.; Plumeré, N.; Rüdiger, O. Viologen-Modified Electrodes for Protection of Hydrogenases from High Potential Inactivation While Performing $\mathrm{H}_{2}$ Oxidation at Low Overpotential. Dalton Trans. 2018, 47, 10685-10691.

(594) Mao, F.; Mano, N.; Heller, A. Long Tethers Binding Redox Centers to Polymer Backbones Enhance Electron Transport in Enzyme "Wiring” Hydrogels. J. Am. Chem. Soc. 2003, 125, 4951-4957.

(595) Prévoteau, A.; Mano, N. Oxygen Reduction on Redox Mediators May Affect Glucose Biosensors Based on "Wired” Enzymes. Electrochim. Acta 2012, 68, 128-133.

(596) Oh, S. E.; Logan, B. E. Voltage Reversal During Microbial Fuel Cell Stack Operation. J. Power Sources 2007, 167, 11-17.

(597) Yoshino, S.; Oike, M.; Yatagawa, Y.; Haneda, K.; Miyake, T.; Nishizawa, M. In 6th World Congress of Biomechanics (WCB 2010). August 1-6, 2010 Singapore: In Conjunction with 14th International Conference on Biomedical Engineering (ICBME) and 5th Asia Pacific Conference on Biomechanics (APBiomech); Lim, C. T.;Goh, J. C. H., Eds. Berlin, Heidelberg, 2010.

(598) Galindo-de-la-Rosa, J.; Arjona, N.; Moreno-Zuria, A.; Ortiz-Ortega, E.; Guerra-Balcázar, M.; Ledesma-García, J.; Arriaga, L. G. Evaluation of Single and Stack Membraneless Enzymatic Fuel Cells Based on Ethanol in Simulated Body Fluids. Biosens. Bioelectron. 2017, 92, 117-124.

(599) Shitanda, I.; Nohara, S.; Hoshi, Y.; Itagaki, M.; Tsujimura, S. A Screen-Printed Circular-Type Paper-Based Glucose/O $\mathrm{O}_{2}$ Biofuel Cell. J. Power Sources 2017, 360, 516-519.

(600) Pan, F.; Samaddar, T. Charge Pump Circuit Design; McGraw Hill Professional: New York, 2006.

(601) Southcott, M.; MacVittie, K.; Halamek, J.; Halamkova, L.; Jemison, W. D.; Lobel, R.; Katz, E. A Pacemaker Powered by an Implantable Biofuel Cell Operating under Conditions Mimicking the Human Blood Circulatory System - Battery Not Included. Phys. Chem. Chem. Phys. 2013, 15, 6278-6283.

(602) Katz, E.; Bückmann, A. F.; Willner, I. Self-Powered Enzyme-Based Biosensors. J. Am. Chem. Soc. 2001, 123, 10752-10753.

(603) Sekretaryova, A. N.; Beni, V.; Eriksson, M.; Karyakin, A. A.; Turner, A. P. F.; Vagin, M. Y. Cholesterol Self-Powered Biosensor. Anal. Chem. 2014, 86, 9540-9547.

(604) Jeerapan, I.; Sempionatto, J. R.; Pavinatto, A.; You, J.-M.; Wang, J. Stretchable Biofuel Cells as Wearable Textile-Based Self-Powered Sensors. J. Mater. Chem. A 2016, 4, 18342-18353.

(605) Wen, D.; Deng, L.; Guo, S.; Dong, S. Self-Powered Sensor for Trace $\mathrm{Hg}^{2+}$ Detection. Anal. Chem. 2011, 83, 3968-3972.

(606) Deng, L.; Chen, C.; Zhou, M.; Guo, S.; Wang, E.; Dong, S. Integrated Self-Powered Microchip Biosensor for Endogenous Biological Cyanide. Anal. Chem. 2010, 82, 4283-4287. 
(607) Wang, T.; Milton, R. D.; Abdellaoui, S.; Hickey, D. P.; Minteer, S. D. Laccase Inhibition by Arsenite/Arsenate: Determination of Inhibition Mechanism and Preliminary Application to a Self-Powered Biosensor. Anal. Chem. 2016, 88, 3243-3248.

(608) Hou, C.; Fan, S.; Lang, Q.; Liu, A. Biofuel Cell Based Self-Powered Sensing Platform for L-Cysteine Detection. Anal. Chem. 2015, 87, 3382-3387.

(609) Conzuelo, F.; Vivekananthan, J.; Pöller, S.; Pingarr ón Jos é, M.; Schuhmann, W. Immunologically Controlled Biofuel Cell as a Self-Powered Biosensor for Antibiotic Residue Determination. ChemElectroChem 2014, 1, 1854-1858.

(610) Gai, P.; Song, R.; Zhu, C.; Ji, Y.; Wang, W.; Zhang, J.-R.; Zhu, J.-J. Ultrasensitive Self-Powered Cytosensors Based on Exogenous Redox-Free Enzyme Biofuel Cells as Point-of-Care Tools for Early Cancer Diagnosis. Chem. Commun. 2015, 51, 16763-16766.

(611) Wang, L.-L.; Shao, H.-H.; Wang, W.-J.; Zhang, J.-R.; Zhu, J.-J. Nitrogen-Doped Hollow Carbon Nanospheres for High-Energy-Density Biofuel Cells and Self-Powered Sensing of MicroRNA-21 and MicroRNA-141. Nano Energy 2018, 44, 95-102.

(612) Zhou, M.; Zhou, N.; Kuralay, F.; Windmiller, J. R.; Parkhomovsky, S.; Valdés-Ramírez, G.; Katz, E.; Wang, J. A Self-Powered "Sense-Act-Treat" System that is Based on a Biofuel Cell and Controlled by Boolean Logic. Angew. Chem. Int. Ed. 2012, 51, 2686-2689.

(613) Gamella, M.; Guz, N.; Pingarron, J. M.; Aslebagh, R.; Darie, C. C.; Katz, E. A Bioelectronic System for Insulin Release Triggered by Ketone Body Mimicking Diabetic Ketoacidosis in vitro. Chem. Commun. 2015, 51, 7618-7621.

(614) Mailloux, S.; Halámek, J.; Katz, E. A Model System for Targeted Drug Release Triggered by Biomolecular Signals Logically Processed through Enzyme Logic Networks. Analyst 2014, 139, 982-986.

(615) Mailloux, S.; Halámek, J.; Halámková, L.; Tokarev, A.; Minko, S.; Katz, E. Biomolecular Release Triggered by Glucose Input-Bioelectronic Coupling of Sensing and Actuating Systems. Chem. Commun. 2013, 49, 4755-4757.

(616) Kohlmann, C.; Märkle, W.; Lütz, S. Electroenzymatic Synthesis. J. Mol. Catal. B: Enzym. 2008, 51, 57-72.

(617) Mazurenko, I.; Etienne, M.; Kohring, G.-W.; Lapicque, F.; Walcarius, A. Enzymatic Bioreactor for Simultaneous Electrosynthesis and Energy Production. Electrochim. Acta 2016, 199, 342-348.

(618) Wu, R.; Zhu, Z. Self-Powered Enzymatic Electrosynthesis of 1-3,4-Dihydroxyphenylalanine in a Hybrid Bioelectrochemical System. ACS Sustainable Chem. Eng. 2018, 6, 12593-12597.

\section{Bios}

Xinxin Xiao is currently a postdoctoral researcher at the Technical University of Demark working with Prof. Jingdong Zhang. He received his Ph.D. in January 2019 at the University of Limerick under the supervision of Prof. Edmond Magner. He was a visiting researcher in 
Prof. Aihua Liu's group at Qingdao University in October 2017. His research interests are focused on the immobilization of enzymes on solid surfaces for bioelectrochemistry studies and the development of unique hybrid devices such as biosupercapacitors.

Hong-qi Xia received his Ph.D. degree at Kyoto University in 2017 under the supervision of Prof. Kenji Kano. He then worked as a research associate at Key Lab of Electroanalytical Chemistry, Changchun Institute of Applied Chemistry, Chinese Academy of Science. He was a visiting researcher at Qingdao University (with Prof. Aihua Liu) in 2018 before he moved to Sun Yat-sen University and conducted his postdoctoral research. His research interests focus on bioelectrocatalysis and its application in biofuel cells, biosensors and bioreactors.

Ranran $\mathrm{Wu}$ received her Ph.D. degree at the Institute of Urban Environment, Chinese Academy of Sciences in 2015. She then undertook postdoctoral research at the Technical University of Demark until 2016. She is currently an assistant professor at Tianjin Institute of Industrial Biotechnology, Chinese Academy of Sciences. Her research interests focus on the bioelectrochemical systems, electroenzymatic synthesis and bio-nanomaterials.

Lu Bai received her B.S. degree in Chemistry from Nankai University in 2008. She obtained her Ph.D. degree in Analytical Chemistry from the Changchun Institute of Applied Chemistry, Chinese Academy of Sciences under the supervision of Prof. Shaojun Dong in 2013. She is presently an associate professor in the Institute for Biosensing at Qingdao University. Her major research interests focus on biofuel cells, biosensors and self-powered devices.

$\mathrm{Lu}$ Yan is currently pursuing his Master's degree under the supervision of Prof. Aihua Liu at the Institute of Life Sciences \& Institute for Biosensing at Qingdao University. He received his bachelor's degree in biology from Yantai University in 2017. His research focuses on microbial surface display based nanomedicine.

Professor Edmond Magner studied at University College Cork (B.Sc. in chemistry) and then obtained his Ph.D. at the University of Rochester under the supervision of Prof. G McLendon. He was a postdoctoral fellow at Imperial College (with Prof. W.J. Albery) and at the Massachusetts Institute of Technology (with Prof. A.M. Klibanov). Subsequently he was a senior research scientist at MediSense, Inc. and at Abbott Laboratories where he worked on the development of biosensors for the detection of glucose and ketones, devices that are still commercially available. In 1997, he joined the academic staff at the University of Limerick and is now Professor Electrochemistry and Dean of the Faculty of Science and Engineering. 
His current research interests are in bioelectrochemistry and biocatalysis with a particular focus on the immobilisation of enzymes on surfaces. To date he has supervised the theses of $22 \mathrm{Ph} . \mathrm{D}$. and $10 \mathrm{M} . \mathrm{Sc}$. researchers and published over 100 papers. He is a member of the Council of the Bioelectrochemical Society,

Dr. Serge Cosnier is currently Research Director at CNRS and director of the Department of Molecular Chemistry at the Grenoble Alpes University (France) where he began his research career in 1983. He received his doctoral degree in Chemistry from the Toulouse University (1982) and was an Alexander von Humbold postdoctoral fellow at the University of Munich, Germany. Cosnier's activity is focused on molecular electrochemistry and bioelectrochemistry for the development of biological sensors, enzymatic fuel cells and bio-nanomaterials based on carbon nanotubes. He has also worked on the development of electrogenerated polymers for applications as organometallic films, biofilms and films with photoactivable, chiral or fluorescent properties applied to bioelectrochemistry. $\mathrm{He}$ has authored over 355 publications (h-index 60), 3 books and holds 25 patents.

Elisabeth Lojou is research director at the CNRS, France. She obtained her degree in engineering from the National School of Chemistry, Rennes, France in 1985 and her $\mathrm{PhD}$ degree from Paris XII University in 1988. After a post-doctoral position in SAFT-Leclanché Company, Poitiers, France where she developed Li/Liquid cathode batteries, and several positions at CNRS, she integrated the Bioenergetic and Protein Engineering laboratory, Marseille (France) leading a group focusing on the functional immobilization of redox enzymes on nanostructured electrochemical interfaces. Her aim is to understand the molecular basis for the oriented immobilization of enzymes on electrochemical interfaces favoring fast electron transfer process. She developed original electrochemical interfaces for catalytic reduction of metals by cytochromes, as well as for catalytic transformations of $\mathrm{H}_{2}$ and $\mathrm{O}_{2}$ by hydrogenases and multi copper oxidases respectively. Recently she designed the first high temperature $\mathrm{H}_{2} / \mathrm{O}_{2}$ enzymatic fuel cell. She has authored over 100 publications. She is currently chair-elect of the Bioelectrochemistry division of the International Society of Electrochemistry, and a member of the Council of the Bioelectrochemical Society.

Zhiguang Zhu is currently a Professor at Tianjin Institute of Industrial Biotechnology, Chinese Academy of Sciences. He received his B.Sc. degree in Biotechnology from Huazhong University of Science and Technology in 2007, and Ph.D. degree in Biological Systems Engineering from Virginia Tech in 2013. His research interests focus on the construction and engineering of bioelectrocatalytic systems using the interdisciplinary approaches of biochemical engineering, bioelectrochemistry, and synthetic biology. 
Dr. Aihua Liu is a Professor and Director of the Institute for Biosensing, Qingdao University (2016-present). Previously he was a Professor at the Key Laboratory of Biofuels, Qingdao Institute of Bioenergy \& Bioprocess Technology, Chinese Academy of Sciences, where he led the Biosensing Group (2010-2016). He received his Ph.D. in Pharmaceutical Physico-chemistry from Tohoku University, Japan in 2004. Then he worked in the National Institute of Advanced Industrial Science \& Technology (AIST) at Tsukuba, Japan under the Japanese Society for the Promotion of Sciences (JSPS) fellowship (2004-2006). He subsequently moved to the US to conduct postdoctoral research at Michigan State University, the University of Oklahoma, and the University of Texas at Arlington (2006-2010). His research interests cover microbial surface display, bioelectrochemistry, biosensors, bioenergy and nanomedicine. He has authored over 80 papers and 3 book chapters.

\section{Graphic TOC}



